\title{
Structural and functional characterization of the autophagy proteins Atg5 and Atg16L1 and their interaction partners
}

\section{PhD Thesis}

in partial fulfillment of the requirements for the degree "Doctor of Philosophy (PhD)" in the Molecular Biology Program at the Georg August University Göttingen, Faculty of Biology

submitted by Amanda Marie Schalk

born in

Toledo, USA

March 2011 
I hereby declare that I prepared the $\mathrm{PhD}$ thesis "Structural and functional characterization of the autophagy proteins Atg5 and Atg16L1 and their interaction partners" on my own and with no other sources and aids than quoted.

Amanda Marie Schalk 


\section{TABLE OF CONTENTS}

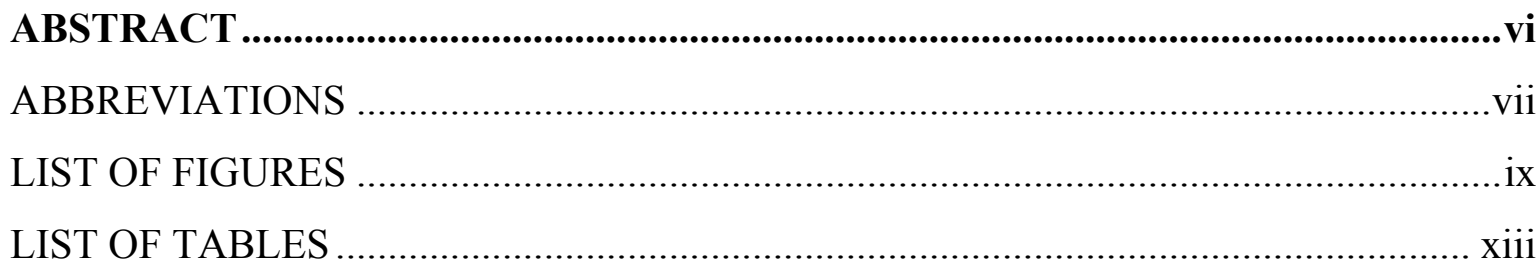

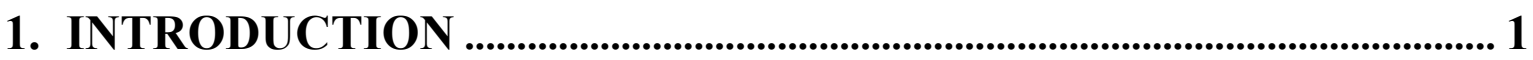

1.1 Autophagy: Types and Functions ........................................................................ 1

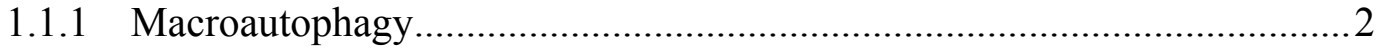

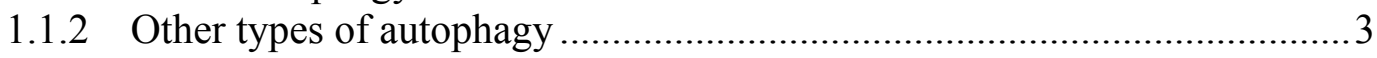

1.2 Autophagosome Formation ...................................................................4

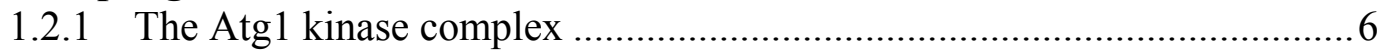

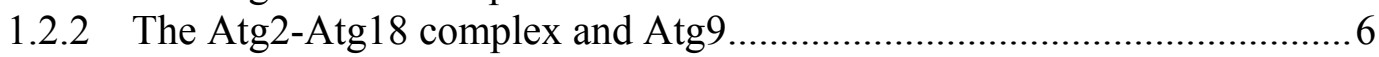

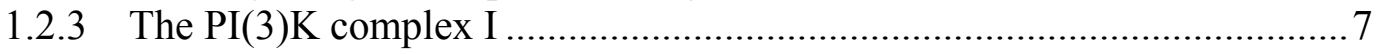

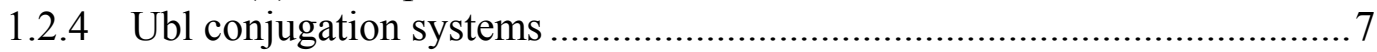

1.3 Additional Interaction Partners of Atg5 ..............................................................13

1.4 Additional Interaction Partners of Atg16L1 ...............................................14

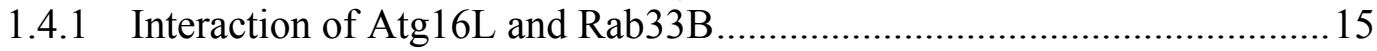

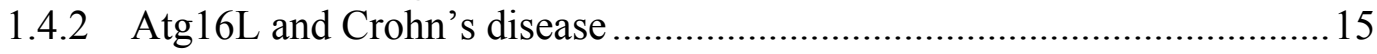

1.5 Localization of Atg5 Atg16 to Autophagosomal Membranes .......................16

1.5.1 Autophagy and phosphoinositides ......................................................... 16

1.5.2 Requirements for membrane recruitment of the Atg5-Atg16 complex .... 17

1.6 Aims of This Work .................................................................................... 19

2. MATERIALS AND METHODS ...................................................................21

2.1 Molecular Biology Techniques..................................................................21

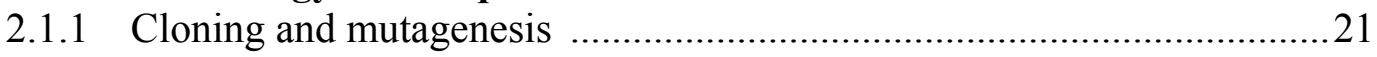

2.1.2 Transformation into competent $E$. coli cells .......................................26

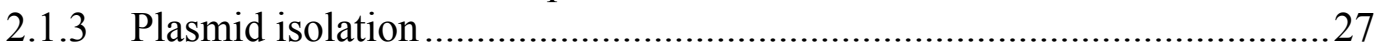

2.1.4 Concentration determination and sequencing of plasmid DNA ..............28

2.2 Protein Expression, Purification, and Detection: General Protocols ................29

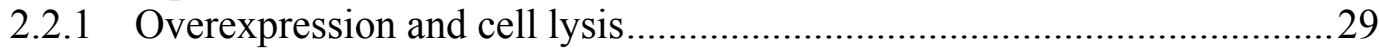

2.2.2 Affinity chromatography and cleavage of the affinity tag ...................... 31

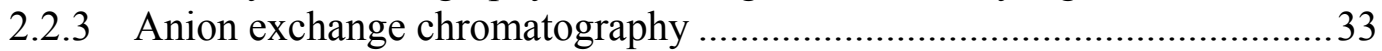

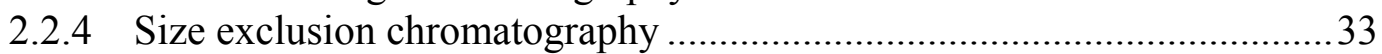

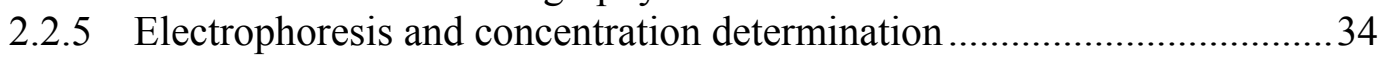

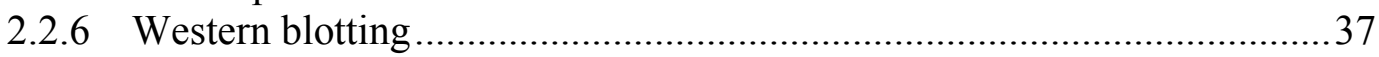

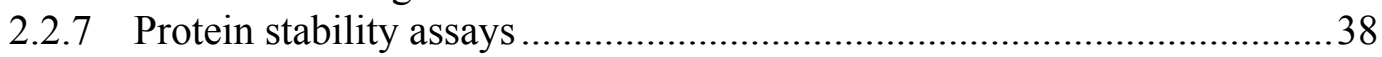

2.3 Protein-specific Purification Protocols................................................................39

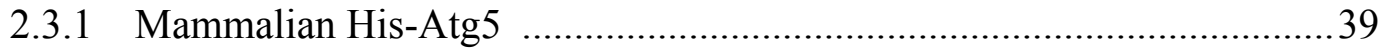

2.3.2 Mammalian His-Atg5 His-Atg16L1 truncation complexes..................... 40

2.3.3 Mammalian His-Atg5 His-Atg16L1(1-623) ....................................... 41 


\section{TABLE OF CONTENTS (continued)}

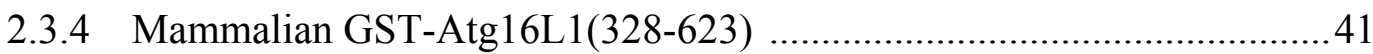

2.3.5 Mammalian GST-Rab33B(1-229) Q92L ............................................... 41

2.3.6 Mammalian His-Rab33B(30-202) Q92L ............................................ 42

2.3.7 Mammalian His-Rab33B(30-202)Q92L $\sim$ Atg16L1 complexes ................ 42

2.3.8 Mammalian His-Atg5 His-Atg16L1(1-265) mutants ............................. 43

2.3.9 Yeast His-Atg5 Atg16(1-46) \& Atg5 Atg16(1-57) complexes ..............44

2.4 Molecular Weight Determination ...................................................................................44

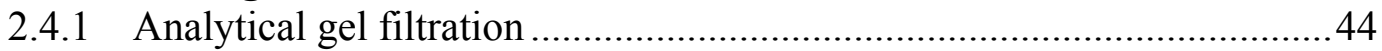

2.4.2 Multi-angle laser light scattering (MALLS) .......................................... 45

2.5 Calpain Cleavage and Fragment Interaction Analysis .....................................46

2.5.1 Calpain cleavage of Atg5 and Atg5 Atg16L1 complexes ......................46

2.5.2 Atg5 interaction analysis of calpain cleavage products ...........................46

2.5.3 Western blot of Atg5 \& Atg5 Atg16L1(1-231) following calpain

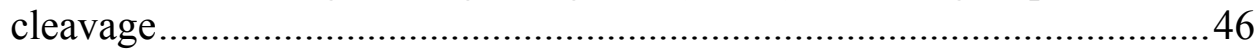

2.6 Complex Formation between Rab33BQ92L and Atg5 Atg16L1 Complexes .. 47

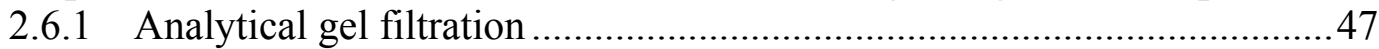

2.6.2 Isothermal titration calorimetry …….................................................47

2.7 Limited Proteolysis and Interaction Analysis via Chromatography.................48

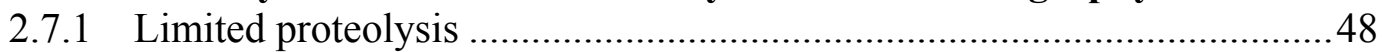

2.7.2 Interaction analysis of cleavage products via chromatography ...............49

2.7.3 Cleavage site determination via N-terminal protein sequencing ...............51

2.8 Crystallization and Structure Determination............................................................51

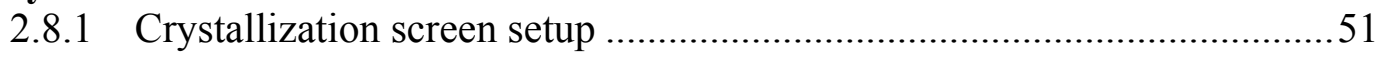

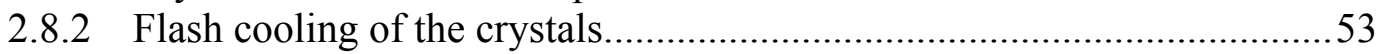

2.8.3 X-ray diffraction data collection and processing ...................................5

2.8.4 Calculation of the Matthews coefficient and self-rotation function .........54

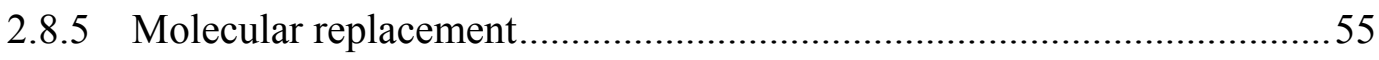

2.8.6 Refinement, density visualization, and generation of figures ..................56

2.9 Characterization of Lipid-Protein Interactions ............................................56

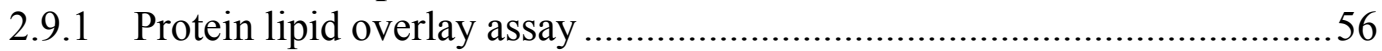

2.9.2 Synthesized Atg16L1(1-25)-StrepII peptide ........................................5

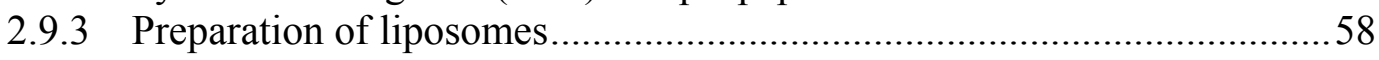

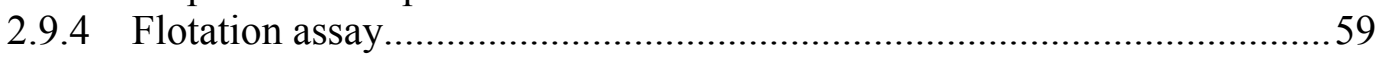

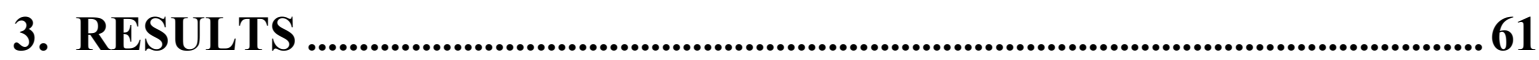

3.1 Characterization of Mammalian Atg5 Atg16L1 Complexes .........................61

3.1.1 Purification of Atg5 Atg16L1 complexes ..............................................6 61

3.1.2 Oligomerization state determination of Atg5 Atg16L1 complexes.........68

3.1.3 Stability test and limited proteolysis experiments of Atg5 Atg16L1(1-

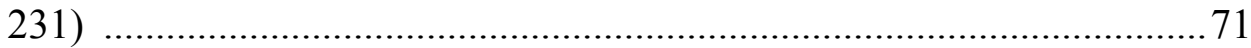

3.1.4 Crystallization of mammalian Atg5 Atg16L1 complexes ….................. 72

3.1.5 X-ray data collection and processing of the Atg5 Atg16L1(1-106) ........77

3.1.6 Cleavage of Atg16L1 by the protease calpain .....................................78

3.2 Characterization of Mammalian Rab33B Atg16L1 Complexes.....................82

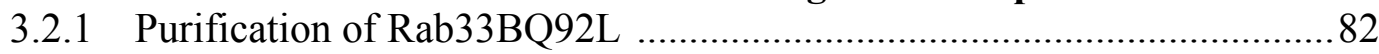




\section{TABLE OF CONTENTS (continued)}

3.2.2 Complex formation of Atg5 Atg16L1 and Rab33BQ92L ....................83

3.2.3 Co-purification of Rab33B Q92L with Atg16L1 constructs .....................86

3.2.4 Limited proteolysis experiments of Rab33B Q92L Atg16L1

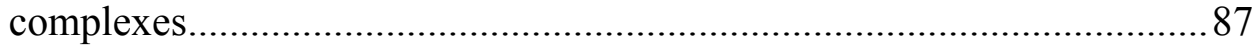

3.2.5 Crystallization of Rab33B(30-202)Q92L $\sim A t g 16 L 1$ complexes............... 94

3.3 Characterization of Phosphoinositide Binding of the Atg5 Atg16

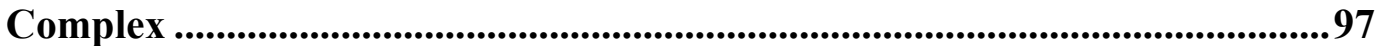

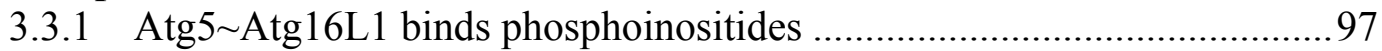

3.3.2 Phosphoinositide binding of the yeast Atg5 Atg16 complex .................99

3.3.3 Dissection of the interaction of Atg5 Atg16 with phosphoinositides .... 102

3.3.4 Atg5 Atg16L1 binds phosphoinositide-containing liposomes.............. 109

3.3.5 Limited proteolysis of Atg5 Atg16L1(1-106) complex with $\operatorname{IP}(1,3)_{2} \ldots 110$

3.3.6 Crystallization of mammalian Atg5 Atg16L1 complexes with inositol

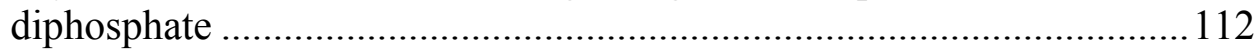

3.3.7 Crystallization and structure determination of yeast Atg5 Atg16 complexes with inositol diphosphate

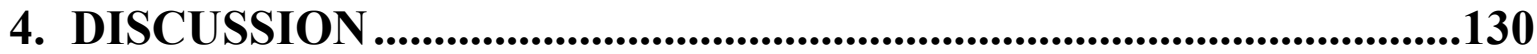

4.1 Characterization of Mammalian Atg5 Atg16L1 Complexes.......................... 130

4.1.1 Oligomerization state determination of mammalian Atg5 Atg16L1

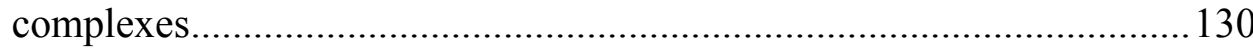

4.1.2 Cleavage of Atg16L1 by the calpain protease .....................................131

4.2 Characterization of Mammalian Rab33B Atg16L1 Complexes.................... 132

4.3 Characterization of Phosphoinositide Binding of Atg5 Atg16

Complexes .................................................................................................................133

4.3.1 Atg5 Atg16(L1) binds phosphoinositides .............................................. 133

4.3.2 Atg5 Atg16(L1) binds PIPs through a non-classical binding domain ......134

4.3.3 Implications of PIP binding for autophagosome formation......................136

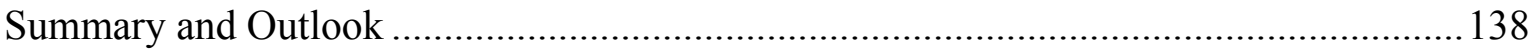

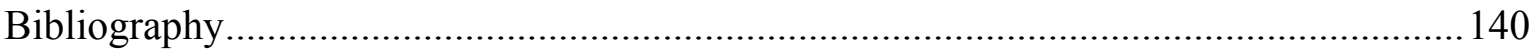

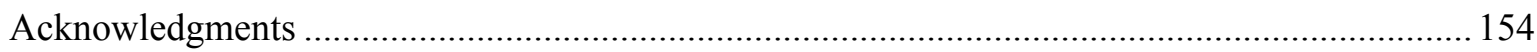

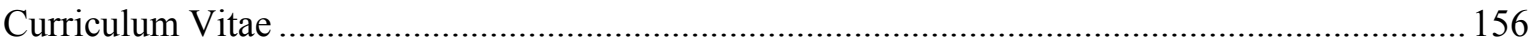




\section{$\underline{\text { ABSTRACT }}$}

Autophagy is a degradative pathway conserved in eukaryotes. During autophagy a portion of the cytoplasm is sequestered by a de novo forming isolation membrane resulting in the formation of a double membrane vesicle. This autophagosome vesicle then fuses with the vacuole, or lysosome in animals, where its content is degraded. The Atg12Atg5 Atg16 complex localizes to the growing isolation membrane and is essential for autophagosome formation. The complex is oligomerized via the coiled coil domain of Atg16 and this oligomerization is required for the expansion step during autophagosome formation.

In this study, the oligomerization state of Atg5 Atg16L1 complexes comprising various lengths of the coiled coil domain of Atg16L1 were investigated with multiple angle laser light scattering. The coiled coil domain homo-dimerizes Atg16L1, and this in turn causes two copies each of Atg5 and Atg16L1 to be present in the complex.

Several interaction partners of Atg5 and Atg16 were also characterized. For example, Atg16L1(141-265) interacts with Rab33B in a GTP-dependent manner (Itoh 2008). In this study it was shown that residues 172-234 of Atg16L1 are sufficient for binding. Crystals of the Rab33B(30-202)Q92L Atg16L1(141-265) complex were grown, however they did not diffract. Furthermore, I showed that calpain cleaves Atg16L1 but not Atg5. Cleavage of autophagy proteins by calpain could play a role in the regulation of autophagy.

Since the Atg12-Atg5 Atg16 complex associates with the isolation membrane, I tested for a direct interaction of the mammalian Atg5 Atg16L1 complex with lipids. Using protein lipid overlay assays and liposome flotation assays, I found that Atg5 Atg16L1 directly binds phosphoinositides, preferentially the phosphatidylinositol monophosphates $\mathrm{PI}(3) \mathrm{P}$ and $\mathrm{PI}(5) \mathrm{P}$, in addition to $\mathrm{PI}(3,5) \mathrm{P}_{2}$ and $\mathrm{PI}(4,5) \mathrm{P}_{2}$. The coiled coil domain of Atg16L1 increases binding affinity. I also demonstrated that PIP binding is evolutionarily conserved, because also the yeast Atg5 Atg16 complex binds PIPs.

The Atg5 Atg16 complex does not resemble any known PIP binding protein. The interaction of the complex with PIPs is mediated by basic residues at the Atg16 Nterminus which are predicted to form an $\alpha$-helix. Mutating these residues to alanines impairs PIP binding. We speculate that the Atg12-Atg5 Atg16 complex could be recruited to the pre-autophagosomal structure and isolation membrane by directly binding to phosphoinositides. 


\section{ABBREVIATIONS}

AMBA

Ams 1

APS

Atg

Atg16L

BAR

$\mathrm{C}$

CV

$\mathrm{Cvt}$

Da

DUB

EEA1

FT

FYVE

GDP

GST

GTP

His

HRP

IMAC

$\mathrm{IP}_{2}$

IPTG

LAMP

LB

MALLS

MCS

Min

MW

MWCO

MTMR3

$\mathrm{Ni}$

$\mathrm{OD}_{600}$

$\mathrm{P}$

PAS

PC

PCR

PE

PEG

PI

$\mathrm{PI}(3) \mathrm{K}$

PIP

prApe1

PROPPIN

PS

PVDF

PX
acrylamide/N,N'-methylene-bis-acrylamide

$\alpha$-mannosidase

ammonium persulfate

autophagy

Atg16-like

Bin, Amphiphysin, Rvs

Celsius

column volume

cytosol to vacuole targeting

dalton

deubiquitinating enzyme

early endosomal autoantigen 1

flow-through

Fab1, YOTB, Vac1, EEA1

guanosine diphosphate

glutathione-S-transferase

guanosine triphosphate

hexahistidine

horseradish peroxidase

immobilized metal affinity chromatography

D-myo-inositol-diphosphate

isopropyl-beta-D-thiogalactopyranoside

lysosome-associated membrane protein

Luria Bertani

multiple angle laser light scattering

multiple cloning site

minute

molecular weight

molecular weight cutoff

Myotubularin-related phosphatase 3

nickel $\left(\mathrm{Ni}^{2+}\right)$

optical density at $600 \mathrm{~nm}$

pellet

preautophagosomal structure/phagophore assembly site

phosphatidylcholine

polymerase chain reaction

phosphatidylethanolamine

polyethylene glycol

phosphatidylinositol

phosphatidylinositol-3-OH kinase

phosphatidylinositol-phosphate

precursor aminopeptidase I

$\beta$-propeller that binds phosphoinositides

phosphatidylserine

polyvinylidene fluoride

Phox homology 


\section{ABBREVIATIONS (continued)}

RT

$\mathrm{S}$

SDS-PAGE

SM

TEMED

TORC1

TB

$\mathrm{Ubl}$

$\mathrm{UV}_{280}$ room temperature

supernatant

sodiumdodecylsulfate polyacrylamide gel electrophoresis

sphingomyelin

tetramethylethylenediamine

Target of rapamycin complex 1

terrific broth

ubiquitin-like

ultraviolet absorbance at $280 \mathrm{~nm}$ 


\section{LIST OF FIGURES}

1.1 Different types of autophagy ………………………………………………......

1.2 Atg proteins required for the various types of autophagy .......................................... 4

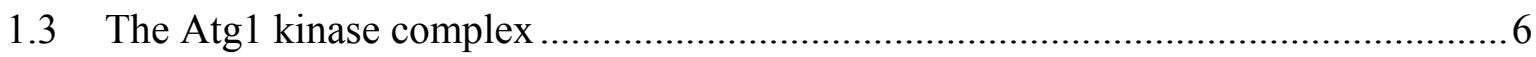

1.4 Atg12 and Atg8 ubiquitin-like (Ubl) conjugation systems ………………............... 8

1.5 Structure of the yeast Atg5 Atg16(1-57) complex ……………………………......

1.6 Yeast Atg16 forms a parallel coiled coil dimer …………………………………..... 10

1.7 Localization of the autophagy Ubl proteins on the isolation membranes ................... 10

$1.8 \mathrm{PI}(3) \mathrm{P}$ is produced in autophagosomal membranes ................................................ 17

1.9 Crystal structures of phosphoinositide binding domains bound to their specific

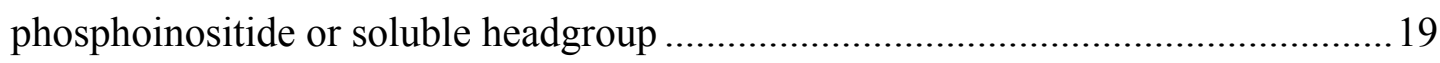

3.1 Ni-sepharose column purification of full-length human His-Atg 5 ............................61

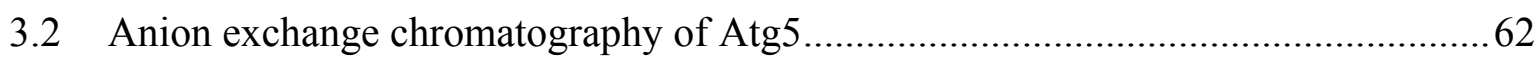

3.3 Constructs of mammalian Atg16L1 for co-purification with full-length

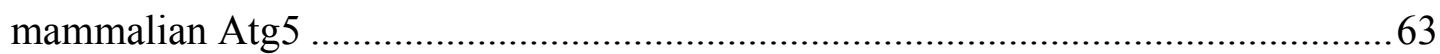

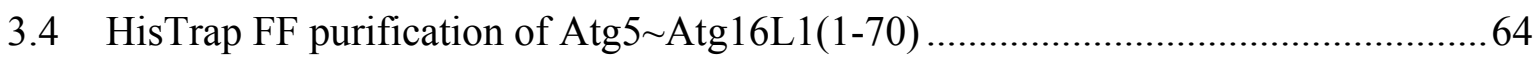

3.5 HisTrap FF purification of Atg5 Atg16L1(1-265) ..............................................64

3.6 Gel filtration purification of Atg5 A Atg16L1 complexes...........................................65

3.7 Gel filtration purification of Atg5 A Atg16L1 complexes.........................................66

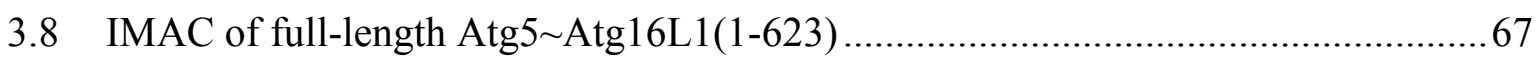

3.9 Gel filtration purification of GST-Atg16L1(328-623) …………………………....68

3.10 Constructs of mammalian Atg16L1 co-purified with Atg5 that were used to determine the oligomerization state of the complex........................................................ 68

3.11 Analytical gel filtration of the Atg5 Atg16L1 complexes ......................................69

3.12 MALLS of Atg16L1(53-168) and Atg5 Atg16L1 complexes ................................70

3.13 Stability test of the Atg5 A $\operatorname{tg} 16 \mathrm{~L} 1(1-231)$ complex at room temperature ….............71

3.14 Limited proteolysis experiments of Atg5 Atg16L1(1-231) ...................................... 72

3.15 Constructs of murine Atg16L1 co-purified with human or murine Atg5 used for crystallization screening .................................................................................. 73

3.16 Crystals of the minimal mammalian Atg5 Atg16L1(1-70) complex ……................74

3.17 Crystals of the oligomerized mammalian Atg5 Atg16L1 complex...........................75 


\section{LIST OF FIGURES (continued)}

3.18 Stereographic projections of the self-rotation function of the Atg5

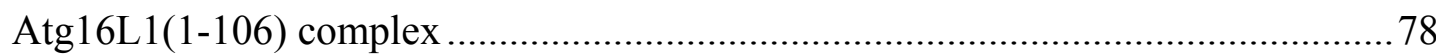

3.19 Calpain-1 cleavage of the Atg5 Atg16L1(1-231) complex ..................................... 79

3.20 Cleavage of the Atg5 Atg16L1(1-231) complex by calpain-1 and calpain-2 ...........80

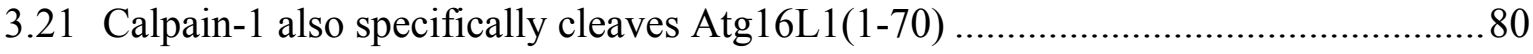

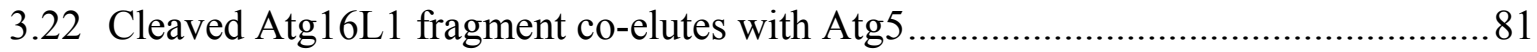

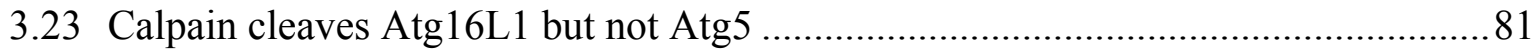

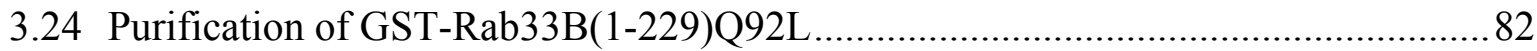

3.25 Purification of murine truncated Rab33B(30-202)Q92L ........................................ 83

3.26 Analytical gel filtration of Rab33B(30-202) Q92L and the Atg5 Atg16L1(1-265) complex .84

3.27 Isothermal titration calorimetry titration curves of Rab33B(30-202)Q92L and the

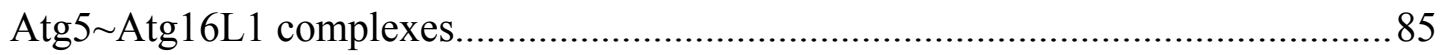

3.28 Co-expression of Atg16L1 with His-Rab33B(30-202)Q92L ....................................86

3.29 Gel filtration purifications of the Rab33B(30-202)Q92L $\sim \operatorname{Atg} 16 \mathrm{~L} 1$ complexes........87

3.30 Limited proteolysis experiments of the Atg5 Atg16L1(1-265) complex and Rab33B(30-202)Q92L

3.31 Ion exchange chromatography of the Rab33B(30-202)Q92L Atg16L1(1-265)

Atg5 complex undigested and digested with trypsin.....

3.32 Cation exchange chromatography of the ternary Rab33B(30-202)Q92L Atg16L1(1-265) Atg5 complex digested with trypsin and the sample sent for Nterminal sequencing... .90

3.33 Limited proteolysis of the Rab33BQ92L Atg16L1(141-265) complex

3.34 Cation exchange chromatography of the Rab33B(30-202)Q92L Atg16L1(141-265) complex and Rab33B(30-202)Q92L alone digested by trypsin

3.35 Anion exchange chromatography of the Rab33B(30-202)Q92L Atg16L1(141-265) complex and Rab33B(30-202)Q92L alone digested by trypsin

3.36 Anion exchange chromatography of the Rab33B(30-202)Q92L Atg16L1(141-265) complex digested with trypsin.

3.37 Purification of His-Rab33B(30-202)Q92L Atg16L1(125-204) ..............................94

3.38 Crystals of the murine His-Rab33B(30-202)Q92L $\sim \operatorname{Atg} 16 L 1(141-265)$ complex .....95 


\section{LIST OF FIGURES (continued)}

3.39 Membrane lipid strip protein lipid overlay assay using His-Atg5 His-Atg16L1(1-70).

3.40 PIP strip protein lipid overlay assay using the Atg5 Atg16L1(1-70) and Atg5 Atg16L1(1-265) complexes.

3.41 PIP array protein lipid overlay assay with mammalian Atg5 Atg16L1 complexes containing varying lengths of the coiled coil domain of Atg16L1

3.42 Yeast Atg5 Atg16L1 complexes used for lipid interaction studies.....

3.43 Purification of the yeast His-Atg5 Atg16(1-46) and His-Atg5 Atg16(1-57) complexes

3.44 PIP strip protein lipid overlay assay using yeast His-Atg5 Atg16 complexes ........ 101

3.45 PIP array protein lipid overlay assay using the yeast His-Atg5 Atg16 complexes ......

3.46 Secondary structure prediction and multiple alignments of Atg16 from yeast to murine

3.47 PIP strip protein lipid overlay assay using the His-Atg5 His-Atg16L1(24-168) complex

3.48 Dot blot assay and protein lipid overlay assay the using Atg16L1(1-25)-StrepII peptide 104

3.49 Scheme of mutations of murine $\operatorname{Atg} 16 \mathrm{~L} 1 \mathrm{~N}$-terminal basic residues. 104

3.50 PIP strip protein lipid overlay assay using mammalian His-Atg5 His-Atg16L1(1-265) wild-type and N-terminal mutant complexes

3.51 PIP strip protein lipid overlay assay using mammalian His-Atg5 His-Atg16L1(1-265) wild type and mutant complexes 106

3.52 Scheme of single, double, and triple mutations of yeast Atg16 N-terminal basic residues to alanines as part of the Atg5 Atg16(1-67) complex 106

3.53 PIP strip protein lipid overlay assays of the yeast His-Atg5 Atg16(1-67) wild-type as well as the single, double, and triple mutant complexes

3.54 Analytical gel filtration of yeast His-Atg5 Atg16(1-67) RKK (wild-type), AKK (R9A), and triple mutant AAA (R9A,K10A,K11A) complexes

$3.5515 \%$ SDS-PAGE gel of the Atg5 Atg16(1-67) wild-type and mutant complexes .. 108 


\section{LIST OF FIGURES (continued)}

3.56 Flotation assay of the murine His-Atg5 His-Atg16L1(1-265) complex with liposomes either with or without PI(3)P

3.57 Flotation assay of the His-Atg5 His-Atg16L1(1-265) complex with liposomes containing $\mathrm{PI}(3) \mathrm{P}$ or $\mathrm{PI}(4,5) \mathrm{P}_{2}$

3.58 Flotation assay of PI(3)P-containing liposomes with the His-Atg5 His-Atg16L1 (1-265) KRR (wild-type) and AAR double mutant (K14A,R15A) complexes....

3.59 Digestions of the murine Atg5 Atg16L1(1-106) complex with or without $\operatorname{IP}(1,3)_{2}$

3.60 Crystals of the mammalian Atg5 Atg16L1(1-70) complex co-crystallized with Dmyo-Inositol-(1,3)-diphosphate (sodium salt) [IP(1,3) 2$]$

3.61 Crystals of the mammalian Atg5 Atg16L1(1-70) complex co-crystallized with $\left.\operatorname{IP}(1,3)_{2}\right]$ and additives .....

3.62 Crystals of the mammalian Atg5 Atg16L1(1-113) and Atg5 Atg16L1(1-106) complexes co-crystallized with IP $(1,3)_{2}$.

3.63 Crystals of the yeast Atg5 Atg16(1-46) complex used for soaking with IP(1,5) 2 or $\mathrm{Na}_{2} \mathrm{SO}_{4}$

3.64 Structure of the yeast Atg5 Atg16(1-46) complex soaked in $\operatorname{IP}(1,5)_{2}$

3.65 Crystals of the yeast Atg5 Atg16(1-57) complex crystallized in the presence of $\operatorname{IP}(1,3)_{2}$

3.66 Structure of the yeast Atg5 Atg16(1-57) complex crystallized in the presence of $\operatorname{IP}(1,3)_{2}$

4.1 Structures of epsin in the presence and absence of the headgroup of $\mathrm{PI}(4,5) \mathrm{P}_{2}$ 135 


\section{LIST OF TABLES}

1.1 Core autophagy machinery proteins and their functional groups .............................5

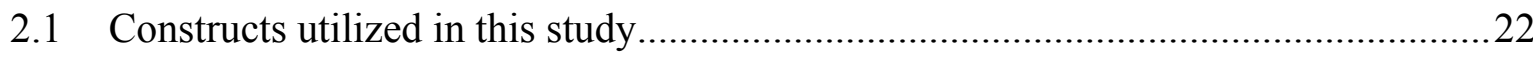

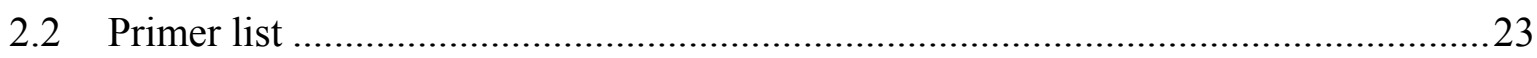

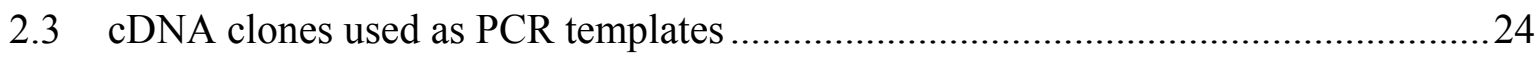

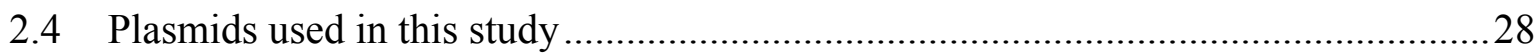

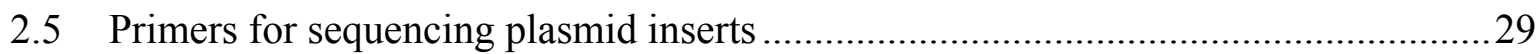

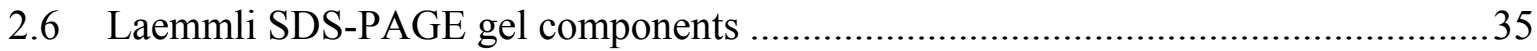

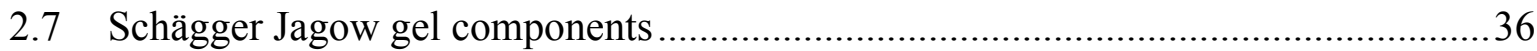

2.8 Natural and synthetic lipids used for liposome preparation ....................................59

3.1 Analytical gel filtration of Atg16L1(53-168) and Atg5 Atg16L1 complexes .........69

3.2 MALLS of Atg16L1(53-168) and Atg5 Atg16L1 complexes .............................. 70

3.3 Initial crystallization conditions for the Atg5 Atg16L1(1-70) complex ...................73

3.4 Optimized crystallization conditions for the Atg5 Atg16L1(1-70) complex ............74

3.5 Crystallization conditions for Atg5 Atg16L1(1-106) and Atg5 Atg16L1(1-113) ... 76

3.6 Cryoprotection and diffraction resolution of Atg5 Atg16L1(1-106) and

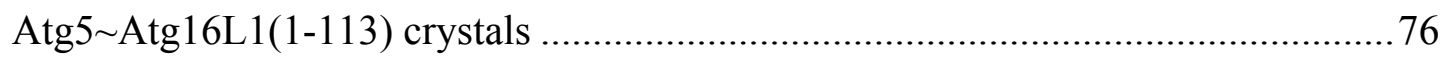

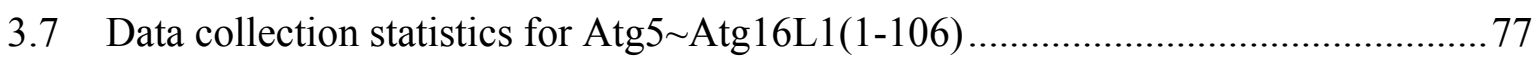

3.8 Matthews coefficient for Atg5 Atg16L1(1-106) crystals .................................... 77

3.9 Crystallization conditions for His-Rab33B(30-202) Q92L $\sim \operatorname{Atg} 16 \mathrm{~L} 1(141-265)$.......96

3.10 Initial screening conditions of mammalian Atg5 Atg16L1(1-70) with $\operatorname{IP}(1,3)_{2} \ldots . .112$

3.11 Crystallization conditions, cryoprotectants, and diffraction resolution data for the 10 best crystals of mammalian Atg5 Atg16(1-70) with IP(1,3)

3.12 Crystallization conditions for mammalian Atg5 Atg16L1(1-106) and Atg5 Atg16L1(1-113) with IP(1,3)

3.13 Crystallization conditions, cryoprotectants, and diffraction resolution data for the 10 best tested crystals of murine Atg5 Atg16L1(1-106) with $\operatorname{IP}(1,3)_{2}$ and

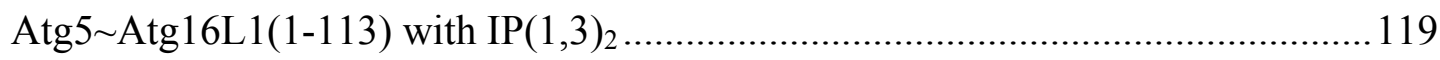

3.14 Diffraction of yeast Atg5 Atg16(1-46) crystals soaked with ligand ....................... 121

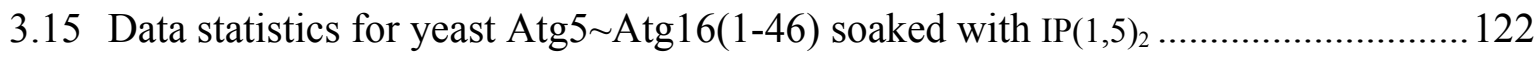

3.16 Crystallization conditions, cryoprotectants, and diffraction resolution data for the

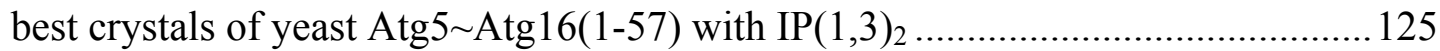

3.17 Data statistics for yeast Atg5 Atg16(1-57) co-crystallized with $\operatorname{IP}(1,3)_{2} \ldots \ldots \ldots \ldots \ldots . . .127$ 


\section{INTRODUCTION}

\subsection{Autophagy: Types and Functions}

The term autophagy (in Greek "self eating") was coined by Christian de Duve in 1963 and is used to describe all catabolic processes whereby cytoplasmic contents are degraded in a lysosome-dependent manner, or in the vacuole in yeast (Nakatogawa 2009, Mizushima 2007, Mizushima 2008, Xie 2007, Klionsky 2007). The morphology and mechanism of autophagy is conserved from yeast to mammals (Nakatogawa 2009, Takeshige 1992). Autophagy genes were discovered by yeast genetic screens (Tsukada 1993, Thumm 1994, Harding 1995), and the first mammalian autophagy genes Atg12 and Atg5 were identified by Mizushima et al. (1998b). Due to an overlap in the naming of genes required for autophagy, the nomenclature of a (Klionsky 2003). Now more than 30 Atg proteins are known (Mizushima 2008). There are three distinct types of autophagy which include macroautophagy, microautophagy, and chaperone-mediated autophagy. In addition, a number of specific subtypes exist. The different forms of autophagy are shown in Figure 1.1 and are discussed in the next sections.

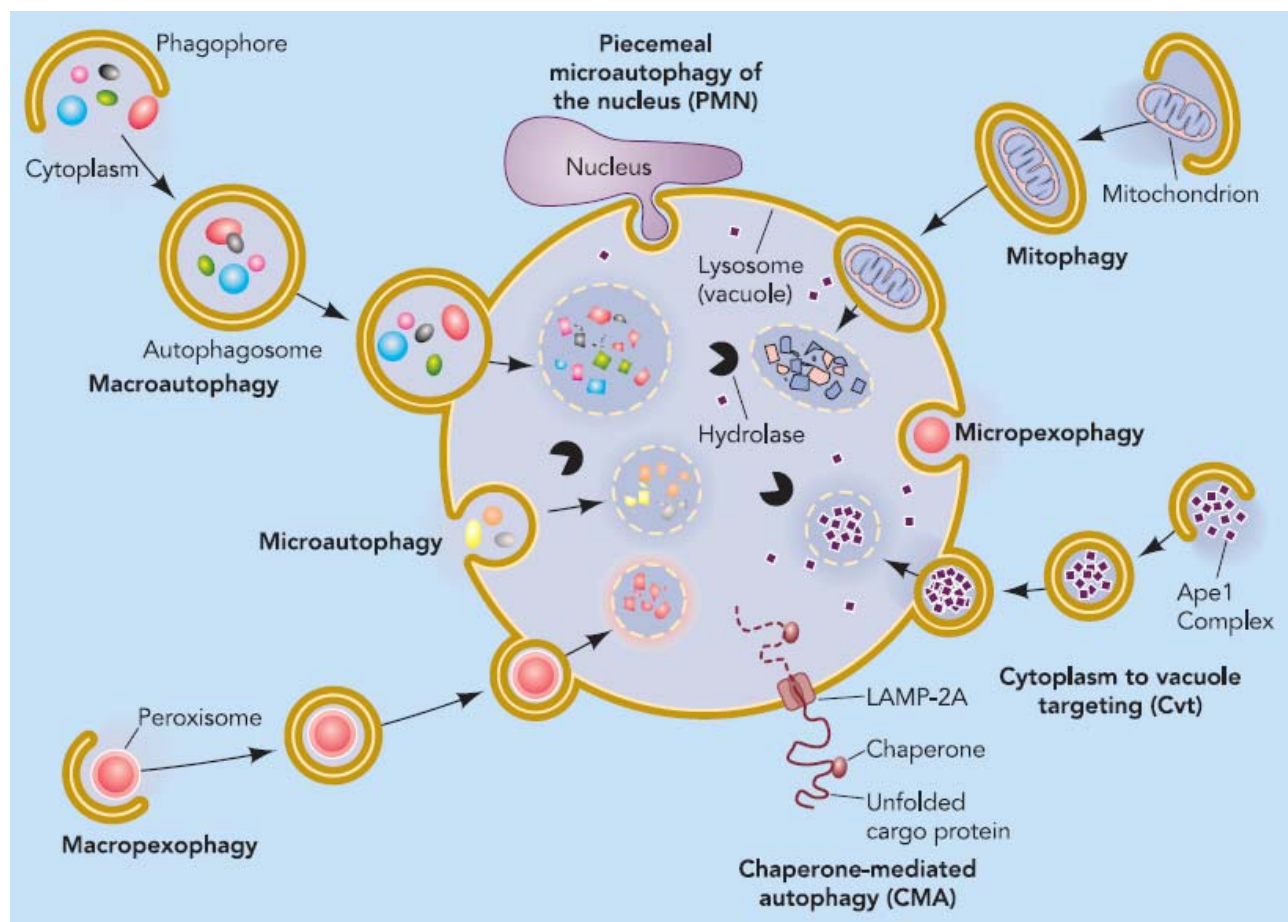

Figure 1.1: Different types of autophagy. A portion of the cytoplasm is sequestered off into a doublemembrane organelle called an autophagosome for bulk degradation upon fusion with the lysosome. In microautophagy, the consumption of cytoplasmic contents is direct through invaginations in the lysosomal membrane. In chaperone-mediated autophagy, proteins are unfolded and translocated across the lysosomal membrane. From Yen \& Klionsky 2008. 


\subsubsection{Macroautophagy}

In macroautophagy, the cytoplasm is sequestered by a de novo growing isolation membrane also called the phagophore. When the leading edges of the isolation membrane fuse, the formation of the autophagosome is complete. In this way, effective sequestration of a portion of the cytosol inside this membrane compartment is accomplished. The outer layer of this double-membrane vesicle fuses with the lysosome. The contents of the autophagic body and the inner membrane of the autophagosome are degraded in the lysosome (Xie 2007).

The origin of the autophagosomal membrane remains a topic of much discussion. Several organelle compartments have been suggested as membrane sources for the isolation membranes: the Golgi complex (Yen 2010, Geng 2010, van der Vaart 2010, Lynch-Day 2010), mitochondria (Hailey 2010), the plasma membrane (Ravikumar 2010), and the endoplasmic reticulum (Axe 2008, Matsunaga 2009, Hayashi-Nishino 2009, YlaAnttila 2009, Matsunaga 2010, Polson 2010). The source of the membrane may be dependent on the nutrient status of the cell and conditions under which autophagy is induced.

Macroautophagy plays many roles in the cell. It is involved in starvation adaptation and metabolism as well as development and differentiation (reviewed in Kroemer 2010, Ravikumar 2009, Yang 2009, Farre 2009). Autophagy is constitutively active at low levels and is important to maintain the homeostasis of long-lived proteins (Komatsu 2005). During macroautophagy, autophagosomes with diameters of 400-900 $\mathrm{nm}$ are formed (Takeshige 1992). This allows the clearance of entire organelles that are damaged or superfluous (Komatsu 2005). Autophagic subtypes exist which target specific organelles for degradation, for example peroxisomes (pexophagy), mitochondria (mitophagy), and the endoplasmic reticulum (ER-phagy) (reviewed in Farre 2009).

An additional important function of macroautophagy is the removal of protein oligomers and large aggregates (Ravikumar 2009, Yang 2009) which are too big to be processed by the ubiquitin-proteasome system which unfolds and degrades ubiquitinated proteins (Ciechanover 2000). Aggregation of mutant forms of huntingtin and $\alpha$-synuclein proteins cause Huntington's disease and familial Parkinson's disease, respectively. Using a Drosophila melanogaster Huntington's disease model, it was shown that stimulating autophagic activity attenuated the toxicity of mutant huntingtin (Ravikumar 2002 and 2004). Neurodegeneration was also observed in mutant mice which were impaired in 
autophagy (Komatsu 2006). Influencing autophagy activity could be a new approach for the treatment of certain neurodegenerative diseases in the future.

Cell death is also affected by macroautophagy. Type II autophagic cell death is characterized by the accumulation of autophagosomes (Bursch 2001). Inhibiting autophagy has an enhancing effect on the amount of cell death in the presence of proapoptotic factors (reviewed in Ravikumar 2009). Impairing autophagy makes cells more susceptible to carcinogenesis. Furthermore, the autophagy protein Beclin 1 acts as a tumor suppressor and tumorigenicity is enhanced in Beclin 1 heterozygous mice (Qu 2003, Yue 2003). Bcl-2 and Bcl- $\mathrm{x}_{\mathrm{L}}$ inhibit autophagy while also having an anti-apoptotic effect (reviewed in Zhou 2011). Other diseases connected to autophagy include muscle atrophy, cardiovascular disease, pancreatitis, liver disease, type II diabetes, and Crohn's disease (Fleming 2011, Komatsu 2010)

In addition, macroautophagy is important to innate immunity as an effector of Tolllike receptor signalling (Beau 2011, Ravikumar 2009, Delgado 2008). This promotes the capture of certain bacteria and viruses in autophagosomes for their subsequent destruction in the lysosome. In fact, certain types of bacteria exploit autophagy and use it as a replicative niche (Levine 2007). Not only is autophagy linked with the innate immune response, but evidence also exists for a role of macroautophagy in the adaptive immune response (reviewed in Beau 2011).

\subsubsection{Other types of autophagy}

During microautophagy, cytoplasmic contents are directly engulfed by the lysosome or vacuole (see Fig. 1.1) (Yang 2009). Whole clusters of peroxisomes can be sequestered in an invagination in the vacuolar membrane before they are engulfed and degraded (micropexophagy) (Farre 2009). During micronucleophagy, otherwise known as piecemeal microautophagy of the nucleus, contacts are generated between the nuclear envelope/ER and the vacuole through the association of the vacuolar protein Vac8 with the ER membrane protein Nvj1. Upon induction by starvation, some of the nucleus pushes through the gate formed by the two ER and vacuolar membrane proteins to form a bud inside the vacuole. The nuclear membrane is cleaved, the vacuolar membranes fuse, and the micronucleus vesicle is degraded (Roberts 2003). Micronucleophagy is dependent on the core autophagy machinery (Krick 2008a).

Chaperone-mediated autophagy also involves a direct uptake of the substrate into the lysosome, but with an entirely different mechanism compared to microautophagy 
(Figure 1.1). One requirement of this pathway is a pentapeptide KFERQ-like motif in the soluble substrate protein that is recognized by the chaperone hsc70 component of the cargo recognition complex (Dice 1990). The unfolded protein is then translocated by the lysosome-associated membrane protein (LAMP) type 2A receptor with the aid of chaperones into the lumen of the lysosome where it is degraded (Dice 2007).

The cytoplasm to vacuole targeting (Cvt) pathway is unique from the other forms of autophagy in that it is a biosynthetic process (Yang 2009, Farre 2009). This selective pathway recognizes its cargo via Atg protein receptors on the Cvt vesicle. The Cvt vesicle specifically enwraps complexes of precursor aminopeptidase I (prApe1) and $\alpha$ mannosidase (Ams1), which are resident vacuolar hydrolases, and delivers them into the vacuole (Figure 1.1).

\subsection{Autophagosome Formation}

In the sections hereafter, macroautophagy will simply be referred to as autophagy. Of the more than 30 identified Atg proteins, about half of them are required for formation of the autophagosome (Suzuki 2010, Nakatogawa 2009, Xie 2007). These are called the core autophagy proteins. The others are required for specific autophagic subtypes, including the Cvt pathway, pexophagy, or mitophagy (see Figure 1.2).

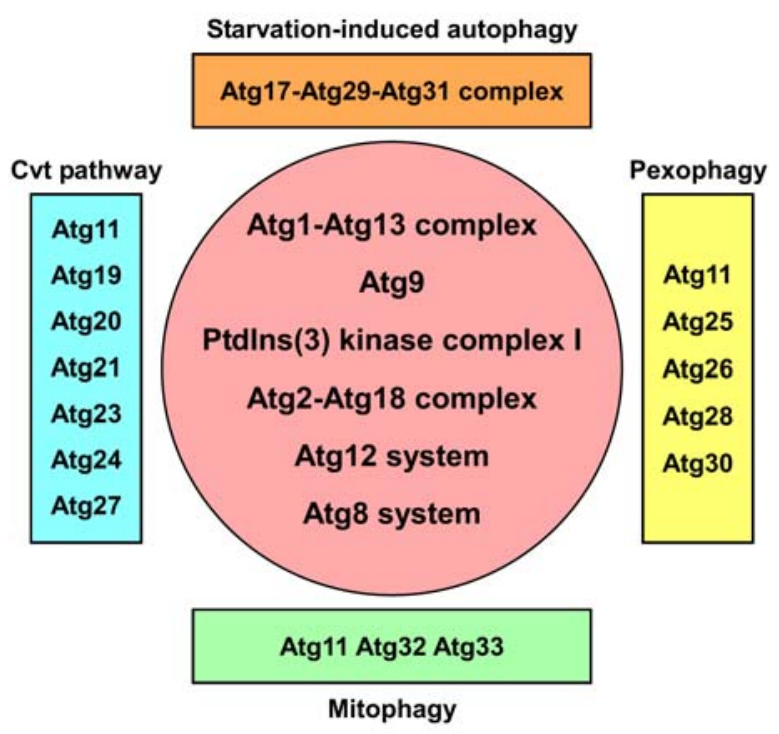

Figure 1.2: Atg proteins required for the various types of autophagy. The core complex Atg proteins required for all types of autophagosome formation are divided into their functional subgroups in the pink circle. The proteins in the orange rectangle are required for initiation of autophagosome formation upon starvation. The Atg proteins in the blue rectangle are required specifically for the biosynthetic Cvt pathway. The proteins in the yellow and green rectangles are required for specific organelle degradation via autophagy. From Suzuki 2010. 
The core machinery proteins are listed in Table 1.1 and can be classified into functional groups: (1) the Atg1 kinase complex, (2) the phosphatidylinositol-3-OH kinase $(\mathrm{PI}(3) \mathrm{K})$ complex, (3) the two ubiquitin-like (Ubl) conjugation systems of Atg12 and Atg8, and (4) the Atg2-Atg18 complex and Atg9. In yeast, most of the core proteins assemble at a perivacuolar site termed the pre-autophagosomal structure or phagophore assembly site (PAS) from which the autophagosome forms (Suzuki 2001). The proteins are recruited to this dot-like structure in a specific order (Cao 2008, Kawamata 2008, Suzuki 2007a,b). In mammalian cells, no single site of Atg protein assembly corresponding to a PAS has been identified. Instead the Atg proteins colocalize at multiple locations (Xie 2007).

Table 1.1: Core autophagy machinery proteins and their functional groups.

\begin{tabular}{|c|c|}
\hline $\begin{array}{l}\text { Subgroup } \\
\text { components }\end{array}$ & Known or putative function \\
\hline \multicolumn{2}{|c|}{ Atg1 kinase and its regulators* } \\
\hline Atg1 & Ser/Thr kinase \\
\hline Atg13 & TORC1 substrate involved in the regulation of Atg1 activity \\
\hline Atg17 & $\begin{array}{l}\text { Forms a starvation-induced autophagy-specific complex with Atg29 and Atg } 31 \text {, } \\
\text { which further associates with Atg1 and Atg13 }\end{array}$ \\
\hline $\operatorname{Atg} 29$ & Component of the Atg17-Atg29-Atg31 complex \\
\hline $\operatorname{Atg} 31$ & Component of the Atg17-Atg29-Atg31 complex \\
\hline \multicolumn{2}{|c|}{ Ptdlns 3-kinase complex ${ }^{\neq}$} \\
\hline Vps34 & Ptdlns 3-kinase \\
\hline Vps15 & Ser/Thr kinase required for $\mathrm{Vps} 34$ activity \\
\hline $\begin{array}{l}\text { Vps30 (also } \\
\text { known as Atg6) }\end{array}$ & Component of unknown function \\
\hline Atg14 & Recruits the Ptdlns 3-kinase complex to the PAS \\
\hline \multicolumn{2}{|c|}{ Atg12 conjugation system 5} \\
\hline Atg12 & Ubiquitin-like protein that is conjugated to Atg 5 \\
\hline Atg 5 & A target of Atg12 and interacts with Atg16 \\
\hline Atg7 & Common E1 enzyme for Atg12-Atg 5 and Atg 8 -PE formation \\
\hline Atg10 & Specific E2 enzyme for Atg12-Atg 5 formation \\
\hline Atg16 & Required for the PAS localization of Atg12-Atg 5 \\
\hline \multicolumn{2}{|c|}{ Atg8 conjugation system" } \\
\hline Atg 8 & Ubiquitin-like protein conjugated to $\mathrm{PE}$ \\
\hline Atg3 & Specific E2 enzyme for Atg8-PE formation \\
\hline Atg 4 & Removes the carboxy-terminal Arg and conjugated PE from Atg 8 \\
\hline Atg7 & Common E1 enzyme for Atg12-Atg 5 and Atg8-PE formation \\
\hline \multicolumn{2}{|c|}{ Atg2-Atg18 complex" and Atg9 } \\
\hline Atg2 & Forms a complex with Atg18, which is involved in Atg 9 dynamics at the PAS \\
\hline Atg18 & Binds to Ptdlns $3 \mathrm{P}$ \\
\hline Atg9 & Integral membrane protein of unknown function \\
\hline
\end{tabular}

Adapted from Nakatogawa et al. (2009). 


\subsubsection{The Atg1 kinase complex}

The Atg17-Atg29-Atg31 complex is present in starvation-induced autophagy and acts as the most upstream scaffold of PAS organization (Suzuki 2010, Kabeya 200 and 2009). The Target of rapamycin complex 1 (TORC1), a regulator of signaling based on nutrient availability, phosphorylates Atg13 (Wullscheger 2006, Noda 1998, Kamada 2000, Funakoshi 1997) (see Figure 1.3). Upon inhibition of TORC1 by rapamycin or starvation, Atg13 is rapidly dephosphorylated and can interact with the Atg1 serine/threonine kinase in this state. The Atg1-Atg13 complex then associates with Atg17 which is part of a ternary complex with Atg29 and Atg31. The Atg1-Atg13 complex then recruits other Atg proteins to the PAS and controls their dynamics (Cheong 2008, Kawamata 2008, Kabeya 2005).

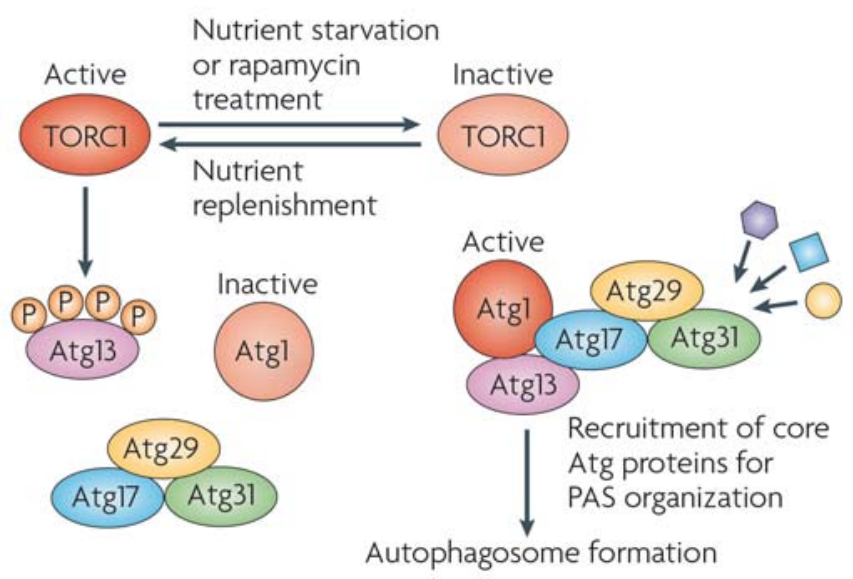

Figure 1.3: The Atg1 kinase complex. Atg13 which is hyperphosphorylated by active TORC1 does not interact with Atg1. Upon inactivation of TORC1, Atg13 is dephosphorylated and interacts with Atg1 and the Atg17-Atg29-Atg31 complex. The Atg1 kinase complex then recruits the other core Atg proteins to the PAS for autophagosome formation. From Nakatogawa 2009.

\subsubsection{The Atg2-Atg18 complex and Atg9}

Atg9 is the only core autophagy integral membrane protein (Noda 2000, Nakatogawa 2009, Xie 2007). Recruitment of the Ubl proteins to the PAS is dependent upon localization of a population of Atg9 there (Suzuki 2001). In addition to PAS localization upon induction, Atg9 is located in dot-like clusters in the cytosol (Noda 2000, Reggiori 2004). Atg9 is not present at mature autophagosomes, and for this reason it was proposed to shuttle from its peripheral compartment, whose nature is still under debate, to the PAS (Reggiori 2004, Reggiori 2005, Young 2006). The shuttling of Atg9 between the PAS and the cytosolic structures is essential for autophagosome formation and in yeast requires Atg23 and the transmembrane protein Atg27 (Reggiori 2006, 2005, 2004; Yen 
2007, Legakis 2007, Tucker 2003). It was suggested that Atg9 shuttling could deliver lipids to the growing isolation membrane (Reggiori 2004, 2005).

Retrieval of Atg9 from the PAS depends on the Atg1 kinase complex, Atg2 and Atg18 (Reggiori 2004, Suzuki 2007a, reviewed in Nakatogawa 2009, Xie 2007). Atg2 and Atg18 form a complex, and Atg9 is able to interact with both of these proteins (Reggiori 2004, Guan 2001, reviewed in Xie 2007). These proteins are recruited to the PAS not only via Atg9 but also require Atg1 and the PI(3)K complex (Guan 2001, Shintani 2001, Wang 2001, Stromhaug 2004, Suzuki 2007a). Atg2 and Atg18 are peripheral membrane proteins. Atg18 directly interacts with phosphatidylinositol-3-phosphate (PI(3)P) and phosphatidylinositol-3,5-bisphosphate $\left(\mathrm{PI}(3,5) \mathrm{P}_{2}\right)$ through a conserved FRRG motif (Dove 2004, Stromhaug 2004, Krick 2006).

\subsubsection{The PI(3)K complex I}

Vps34 is a class III PI(3) kinase and the only one present in yeast (Schu 1993). It plays vital roles in both autophagy and vacuolar protein sorting and exists in separate complexes for each of those functions (Obara 2008, Kihara 2001, Petiot 2000, Schu 1993, reviewed in Xie 2007). Complex I functions in autophagy and is comprised of Vps34, Vps15, Vps30 (otherwise known as Atg6), and Atg14. Atg14 is responsible for localization of the complex to the PAS (Obara 2006, reviewed in Xie 2007). The PI(3)K complex I is responsible for the phosphorylation of phosphatidylinositol (PI) in the membrane at its 3' hydroxyl group to form PI(3)P (Burman 2010). The formation of $\mathrm{PI}(3) \mathrm{P}$ is a requirement for autophagosome formation (Obara 2008, Kihara 2001, Petiot 2000, reviewed in Xie 2007). Additionally, the PI(3)K complex I is essential for localization of the Atg18-Atg2 complex to the PAS (Suzuki 2007a). Autophagy can be inhibited through the use of 3-methyladenine or wortmannin, which both block the production of PI(3)P (Kovacs 2000, Petiot 2000, Blommaart 1997, Seglen 1982).

\subsubsection{Ubl conjugation systems}

There are two ubiquitin-like conjugation systems present in autophagy. The Atg12 and Atg8 systems are essential for formation of the autophagosome (Figure 1.4) (reviewed in Noda 2009). 


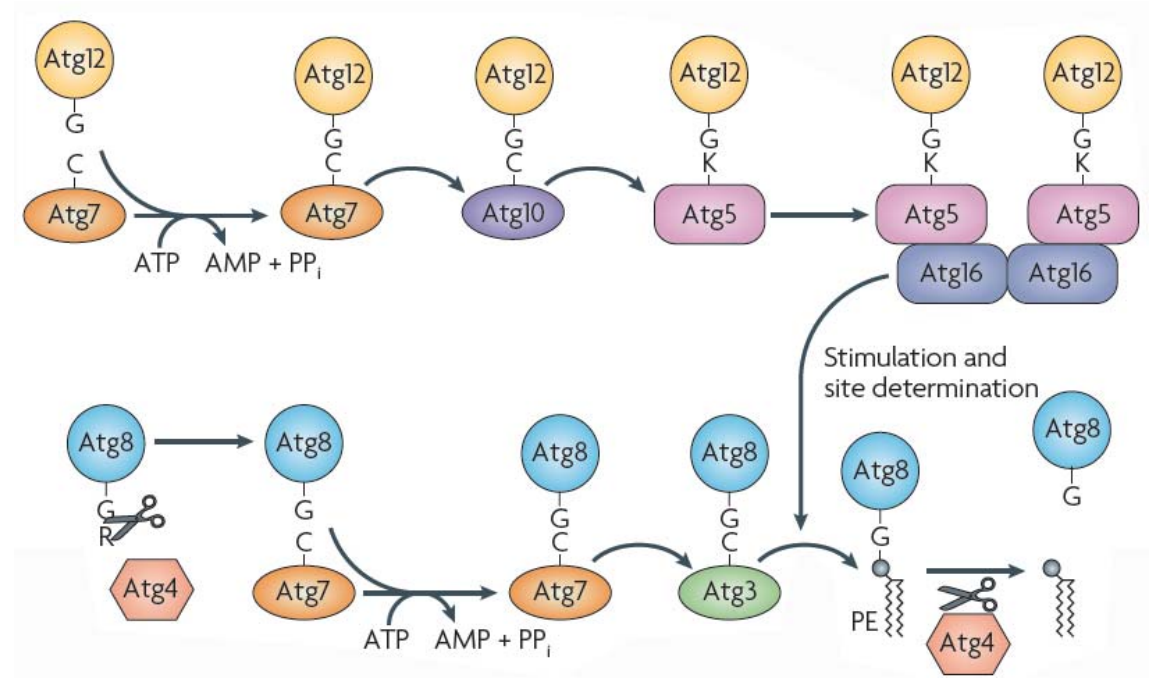

Figure 1.4: Atg12 and Atg8 ubiquitin-like (Ubl) conjugation systems. Atg12 is conjugated to Atg5 in an ATP-dependent manner via Atg7 and Atg10, its E1 and E2-like enzymes, respectively. Atg5 interacts non-covalently with Atg16, whose coiled coil domain dimerizes the complex. Atg8 is conjugated to phosphatidylethanolamine (PE) via Atg7 and Atg3, its E1 and E2-like enzymes, respectively. The Atg12Atg5 Atg16 complex functions as the E3 ligase for the transfer of Atg8 from Atg3 to PE. Atg4 is the deubiquitinating enzyme of Atg8 which processes its C-terminus and makes its conjugation to PE reversible. Modified from Nakatogawa 2009.

\section{Atg12 Ubl conjugation system}

For ubiquitin and Ubl conjugation, a glycine residue is required at the C-terminus of the substrate. Unlike ubiquitin and many Ubls, Atg12 ends with a glycine residue at it $\mathrm{C}$-terminus and therefore requires no initial processing by an Ubl-specific protease (Geng 2008a, Kerscher 2006). Atg12 is comprised of a canonical Ubl fold and is structurally similar to Atg8 homologues (Suzuki 2005). Atg7 is a homodimer which shares homology with E1 enzymes around its ATP binding and active cysteine sites (Komatsu 2001). It functions as the E1-like activating enzyme for Atg12 and requires ATP (Komatsu 2001, Mizushima 1998a). In this way a high-energy thioester bond is formed between the active site cysteine residue in Atg7 and the C-terminal glycine residue of Atg12 (Tanida 1999, Yuan 1999). Atg12 is then transferred to the E2-like conjugating enzyme Atg10 through the formation of another thioester bond (Shintani 1999). There is no E3-like enzyme in the Atg12 conjugation system. After transfer to $\operatorname{Atg} 10, \operatorname{Atg} 12$ is conjugated to $\operatorname{Atg} 5$ via isopeptide bond formation between the C-terminal Gly 186 of yeast Atg12 and the $\varepsilon$-amino group of a Lys149 of yeast Atg5. Conjugation of Atg12 to Atg5 is both constitutive and irreversible (Mizushima 1998a). The Atg12 conjugation system is conserved from yeast to mammals (Mizushima 2002, Tanida 2001, Mizushima 1998b).

Atg5 interacts non-covalently with Atg16 (Atg16L in plants and mammals) (Mizushima 2003, 1999). The structure of yeast Atg5 was solved in complex with the N- 
terminus of yeast Atg16 (Figure 1.5) (Matushita 2006, 2007). Atg5 has a globular fold and consists of two Ubl domains (five-stranded $\beta$-sheet and two $\alpha$-helices), one at each termini, and an $\alpha$-helix rich domain in between. An additional $\alpha$-helix present at the Nterminus $(\alpha 1)$ is important for maintaining Atg5 architecture. Residues 22-40 of Atg16 form an $\alpha$-helix which binds to the groove at the interface between the two Ubl domains and $\alpha 1$ of Atg5. The Atg16 linker region comprising residues 41-57 localizes mainly to the N-terminal Ubl domain of Atg5.
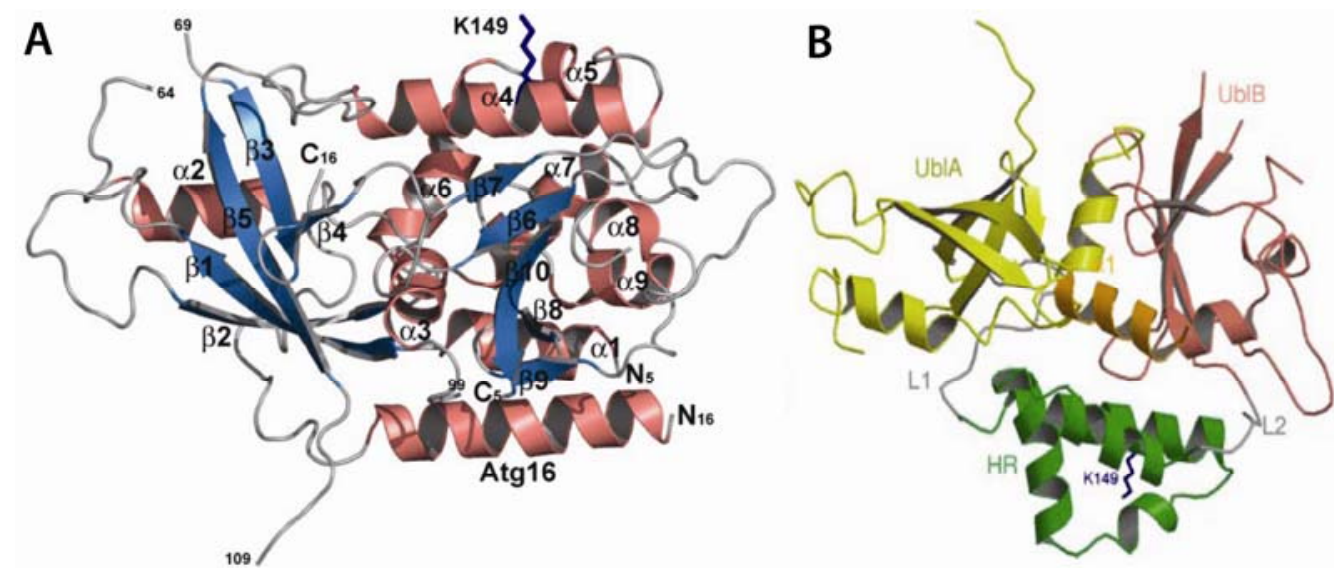

Figure 1.5: Structure of the yeast Atg5 Atg16(1-57) complex . (A) Structure of yeast Atg5 Atg16(157) (PDB ID: 2DYO) with N and C termini denoted by 5 for Atg5 and 16 for Atg16. (B) Domain architecture of Atg5. K149 is the lysine onto which Atg12 is conjugated. Modified from Matsushita 2007.

Yeast Atg16 contains a coiled coil domain (residues 58-123) which is important for its function. Generally, coiled coil proteins consist of at least two and up to five $\alpha$ helices, which are wrapped around each other, usually forming a left-handed superhelix. Sequences of coiled coil proteins are characterized by the occurrence of heptad-repeats (abcdefg) $)_{n}$, where positions a and $\mathrm{d}$ are occupied by hydrophobic amino acids (Lupas 1996).

Homo-oligomerization of the coiled coil domain of Atg16 is responsible for multimerization of the Atg12-Atg5 Atg16 complex and is absolutely required for autophagosome formation (Mizushima 2003, Kuma 2002, Mizushima 1999). Using size exclusion chromatography, it was initially shown that the yeast Atg12-Atg5 Atg16 complex forms a $\sim 350 \mathrm{kDa}$ tetrameric complex and that the murine Atg12-Atg5 Atg16L1 multimeric complex has a molecular weight (MW) of $\sim 800 \mathrm{kDa}$, corresponding to an octameric complex (Kuma 2002, Mizushima 2003). While this study was underway, data showing dimerization of Atg16 was published. Sucrose density gradient experiments by Fujita et al. (2009) indicated the oligomerization state of mammalian Atg16L to be a 
dimer. Fujioka et al. (2010) observed dimerization of the yeast Atg16 coiled coil domain in solution via analytical ultracentrifugation experiments and determined the crystal structure of the coiled coil domain of Atg16. Atg16 forms a dimeric parallel coiled coil (Figure 1.6) (Fujioka 2008, 2010). This would lead to an Atg12-Atg5 Atg16 complex comprised of two copies of each protein.

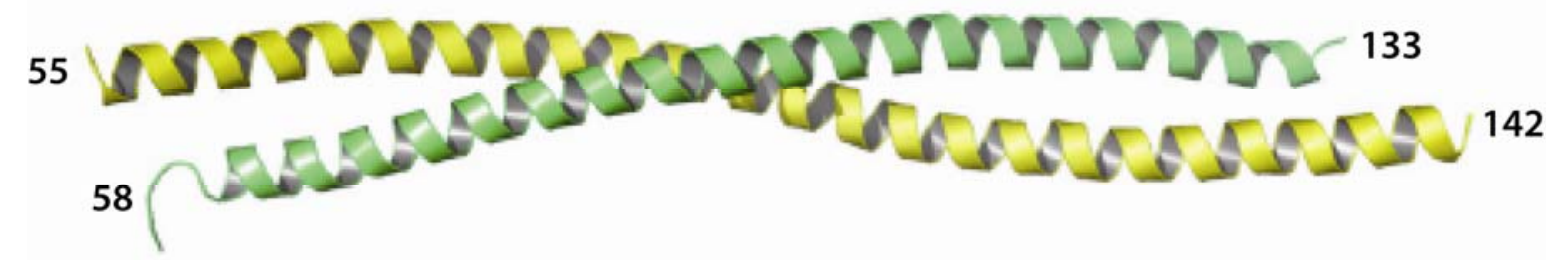

Figure 1.6: Yeast Atg16 forms a parallel coiled coil dimer. Crystal structure of the coiled coil domain of Atg16. (PDB ID: 3A7P) Modified from Fujioka 2010.

The Atg12-Atg5 Atg16 complex is essential for the expansion of the growing isolation membrane. The complex is primarily localized to the outer surface of the expanding isolation membrane and dissociates from the membrane shortly before or after fusion of the leading edges to form the mature autophagosome (Figure 1.7) (Mizushima $2003,2001)$. It was proposed to function as a coatomer complex to induce curvature during autophagosome formation; however, a quantitative study by Geng et al. (2008b) indicates that there are too few molecules of the Atg12-Atg12 Atg16 complex to cover the forming autophagosome.

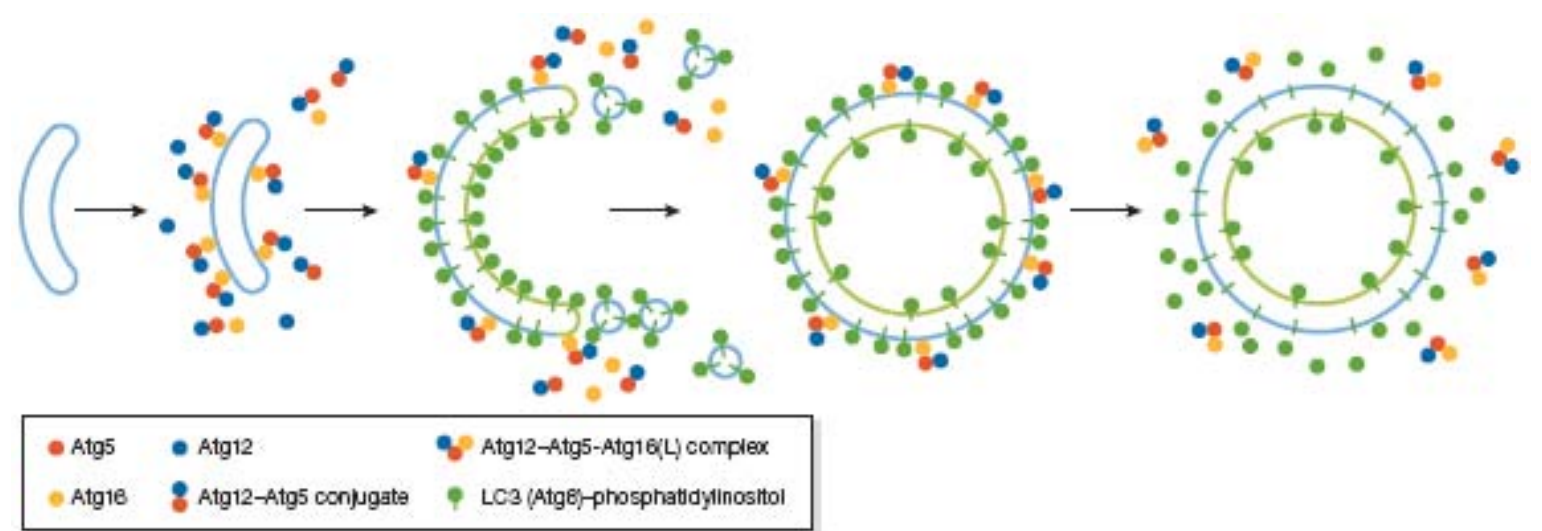

Figure 1.7: Localization of the autophagy Ubl proteins on the isolation membranes. The Atg12Atg5 Atg16 complex is localized mainly to the outer surface of the growing isolation membranes and dissociates shortly before or after autophagosome formation. Atg8 conjugated to PE is bound on both the inner and outer surface and remains trapped inside the autophagosome after fusion of the leading edges. From Geng 2008a. 


\section{Atg8 Ubl conjugation system}

Atg8 and its mammalian homologues are Ubl proteins with an additional Nterminal $\alpha$-helical domain (Mann 1994, Wang 1999, Paz 2000, Sagiv 2000, Hemelaar 2003, Noda 2008). Conjugation of yeast Atg8 begins by the proteolytic cleavage of its Cterminal arginine residue to expose the glycine residue needed for its conjugation to the phospholipid phosphatidylethanolamine (PE). Atg4 (Atg4B in mammals) is a cysteine protease comprised of an $\alpha / \beta$ fold which is structurally similar to papain family cysteine proteases including deubiquitinating enzymes (DUBs) (Kabeya 2004, Tanida 2004, Sugawara 2005, Kumanomidou 2006). Atg4 regulates Atg8 lipidation by performing the initial processing of precursor Atg8 (Kirisako 2000). Atg8 is then activated by the E1-like enzyme Atg7 in an ATP-dependent manner and a thioester bond is formed between the thiol group of the active site cysteine and the C-terminal carboxylate group (Ichimura 2000, Tanida 1999, Kim 1999). Atg8 is then transferred to Atg3, its specific E2-like conjugating enzyme (Ichimura 2000, Schlumpberger 1997). The carboxyl glycine of Atg8 then forms an amide bond with PE (Ichimura 2000). This lipidation of Atg8 is reversible since Atg4 can act as the DUB and cleave Atg8 from the surface of the autophagosomal membrane (Kirisako 2000). Also, in contrast to the irreversible conjugation of Atg12 to Atg5, Atg8 remains primarily unlipidated under nutrient rich conditions and is only lipidated upon induction of autophagy (Huang 2000, Mizushima 1998a).

In recent years, evidence has amassed which points to the $\operatorname{Atg} 12-\operatorname{Atg} 5$ conjugate functioning as a non-classical E3-like ligase for Atg8 by directly interacting with Atg3 and facilitating its transfer to PE (Fujioka 2008, Hanada 2007). When Atg12-Atg5 Atg16 complex formation is genetically inhibited, Atg8 lipidation is greatly reduced (Hanada 2005, Suzuki 2001) Atg12 interaction with Atg3 is conserved from yeast to mammals (Hanada 2007, Tanida 2002). In addition, the Atg12-Atg5 conjugate not only binds liposomes which contain PE but also stimulates the conjugation of Atg8 to PE (Hanada 2007, Fujioka 2008).

In yeast, the $A \operatorname{tg} 12-A \operatorname{tg} 5 \sim A \operatorname{tg} 16$ complex is required for recruitment of $A \operatorname{tg} 8$ to the PAS (Suzuki 2001). Fujita et al, (2008a) showed that in mammalian cells, Atg16L determines the site of lipidation for the mammalian Atg8 homologue, LC3. Ectopic expression of plasma membrane-targeted Atg16L1 caused lipidation of Atg8 on that membrane. Additionally, they showed that Atg16L1 is essential for recruitment of Atg3 to 
PE-containing membranes by the Atg12-Atg5 Atg16 complex, further aiding in the lipidation of PE (Fujita 2008a).

At the PAS, Atg8-PE fulfills various roles. It is essential for membrane expansion and is thought to contribute to regulating the size of the autophagosome. In vitro experiments by Nakatogawa et al. (2007) showed that Atg8-PE oligomerizes and causes liposome clustering and hemifusion. Mutants that are impaired in this function are also impaired in autophagosome formation. Atg8 mutants with partial impairment yielded autophagosomes which were smaller in size. The same effects occur when expression of Atg8 is genetically reduced (Xie 2008). This role for Atg8 was also shown for its mammalian homologues. Disruption of LC3 lipidation leads to a morphology akin to that seen in yeast with an increase in the number of unclosed isolation membranes (Fujita 2008b, Sou 2008).

\section{Other functions of Ubl proteins Atg8 and Atg12-Atg3}

In addition to its essential role in isolation membrane expansion and fusion, Atg8 also plays a role in selective autophagy. In yeast, Atg8 recognizes Atg19, the cargo receptor for prApeI and Ams1, and in combination with Atg11 acts as a link between the receptor and the Cvt vesicle (Scott 2001, Shintani 2002, Yorimitsu 2005). In mammalian cells, LC3 interacts with p62, a cargo receptor for ubiquitylated protein aggregates, allowing for specific degradation of these aggregates via autophagy (Bjorkoy 2005, Pankiv 2007, Komatsu 2007). Atg8 as well as its lipidated form, interact with Shp1, a cofactor of the Cdc48 AAA-ATPase protein, both of which have recently been identified as novel proteins essential for autophagosome formation (Ju 2009, Tresse 2010, Krick 2010, Thumm 2011).

Atg5 is not the sole conjugation target of Atg12 and its new function is distinct from its role during autophagosome formation. Radoshevich et al. (2010) showed that after activation of $\operatorname{Atg} 12$ by $\operatorname{Atg} 7, \operatorname{Atg} 12$ can be linked to a lysine in Atg3, the E2-like enzyme in the Atg8 Ubl conjugation pathway. The Atg12-Atg3 conjugate comprises only a fraction of the total pool of endogenous Atg3. Atg12-Atg3 does not play a role in isolation membrane expansion and autophagosome maturation but rather is involved in mitophagy, mitochondrial-mediated cell death, and mitochondrial homeostasis (Radoshevich 2010, 2011). 


\subsection{Additional Interaction Partners of Atg5}

Apart from the essential function they exert on isolation membrane expansion during autophagosome formation, both Atg5 and Atg16 have been implicated in other cellular processes and linking them to autophagy. In addition to being part of the core autophagy machinery, several observations connect Atg5 to cell death.

Yousefi et al. (2006) found that Atg5 sensitized cells to various apoptotic stimuli and that this occurs through cleavage of Atg5 to a truncated $24 \mathrm{kDa}$ cleavage product comprising residues 1 to 193 by the proteases calpain-1 and calpain-2. Calpains are cysteine proteases which require $\mathrm{Ca}^{2+}$ for activation (Goll 2003). Calpain-1 and calpain-2 are ubiquitously expressed and are also referred to as micro $(\mu)$ and milli $(\mathrm{m})$ calpain, respectively, in accordance with the amount of $\mathrm{Ca}^{2+}$ required for proteolytic activity (Goll 2003, Cong 1989). Calpain-1 and -2 are heterodimers consisting of a large $\sim 80 \mathrm{kDa}$ subunit and a smaller $\sim 28 \mathrm{kDa}$ subunit and are physiologically inhibited by the protein calpastatin which is specific for calpains (Goll 2003). The Atg5 calpain cleavage product translocates from the cytosol to the mitochondria where it interacts with the anti-apoptotic protein $\mathrm{Bcl}-\mathrm{x}_{\mathrm{L}}$. Truncated Atg5 triggers the release of cytochrome $c$ either alone or together with other pro-apoptotic proteins (Yousefi 2006). This then causes activation of caspase-3 and mediates apoptosis.

It was also reported that Atg5 mediates interferon- $\gamma($ IFN- $\gamma$ ) induced cell death (Pyo 2005) by interacting with Fas-associated protein with death domain (FADD). Pyo et al. (2005) showed that IFN- $\gamma$ cell death was suppressed upon down-regulation of Atg5 in HeLa cells and ectopic expression of Atg5 was sufficient to induce cell death.

Xia et al. (2010) have implicated the calpain cleavage of Atg5 as a regulatory mechanism for autophagy. The small molecule fluspirilene induces autophagy through the reduction of intracellular $\mathrm{Ca}^{2+}$ (Xia 2010, Zhang 2007). Xia et al. (2010) showed that fluspirilene increased levels of full-length Atg5 and the Atg5-Atg12 conjugate by inhibiting calpain cleavage of Atg5. They demonstrated that inhibition of calpain, and its cleavage of Atg5, was sufficient to induce autophagy.

Atg5 plays a role in both the enhancement and destruction of microbial replication in host cells via the innate immune response. Atg5, as part of the Atg5-Atg12 conjugate, contributes to RNA virus replication in host cells by associating with the retinoic acidinducible gene 1 , an RNA helicase which detects virus-derived RNA and interferon- $\beta$ promoter stimulator 1 , an adaptor molecule required for activation of type I interferon 
signalling as an innate immune response (Jounai 2007). In contrast, Atg5 binds to VirG, an outer surface protein of Shigella flexneri, which is required for actin-based motility (Ogawa 2005). As a survival mechanism, the bacterium secretes a second protein, IcsB, which blocks the Atg5-binding site on VirG. Consequently, the bacterium is no longer recognized by Atg5 and its degradation by autophagy is prevented.

In a proteomics study conducted by Behrends et al. (2010), Atg5 was also shown to interact with Atg3, and tectonin beta propeller repeat containing protein 1 (TECPR1) which contains a PH domain and the transketolase TKT.

\subsection{Additional Interaction Partners of Atg16}

Yeast Atg16 and mammalian Atg16-like (Atg16L) proteins differ in their domain structures. Whereas Atg16 in yeast is 150 amino acids long and is comprised of an Nterminal Atg5-binding domain (residues 1-46) and a coiled coil domain at its C-terminus (residues 58-123) (Mizushima 1999, Kuma 2002, Matsushita 2007, Fujioka 2010), mammalian Atg16L has an additional C-terminal WD40-repeat domain and is three to four times larger than yeast Atg16, depending on the isoform (Mizushima 2003). Murine Atg16L1 was identified by Mizushima et al. (2003) and has three splice variants: $\operatorname{Atg} 16 \mathrm{~L} \alpha, \operatorname{Atg} 16 \mathrm{~L} \beta$, and $\operatorname{Atg} 16 \mathrm{~L} \gamma$. The liver, kidney, spleen, thymus, and testes contained a major form and a minor form: Atg16L $\alpha$ is the major form and lacks exons 8 and 9 (588 total residues) and $\operatorname{Atg} 16 \mathrm{~L} \beta$ is the minor form and lacks exon 9 (607 total residues). Atg16L $\gamma$ (623 total residues) is the major species present in the brain, skeletal muscle and heart and contains the complete sequence for exons 8 and 9 which lie between the coiled coil domain and the WD40 repeat domain (Mizushima 2003). The WD40 repeat motif forms a $\beta$-propeller which often mediates protein-protein interactions (Smith 1999). Mizushima et al. (2003) postulated that the additional WD40-repeat domain present in mammalian Atg16L could facilitate autophagic processes specific to higher eukaryotes.

Atg16L1 plays a role in plasma membrane endocytosis by associating with clathrin heavy-chain and to a lesser degree, the adaptor protein AP2 (Ravikumar 2010). These Atg16L-clathrin coated vesicles originate from endocytosed plasma membrane and this interaction is essential for formation of autophagosomal precursors as well as mature autophagosomes. 


\subsubsection{Interaction of Atg16L and Rab33B}

$\operatorname{Atg} 16 \mathrm{~L}$ is an effector of a Rab33B (Itoh 2008). Rab proteins are small GTPases which regulate membrane trafficking events (reviewed in Stenmark 2009). GTPases bind guanosine triphosphate (GTP) and have an intrinsic GTPase activity whereby they hydrolyze the GTP to guanosine diphosphate (GDP). GTPases cycle between an active GTP-bound state and an inactive, GDP-bound state. Several proteins aid in this transformation. GTPase activating proteins accelerate the hydrolysis of GTP to GDP by stimulating the intrinsic GTPase activity of the GTPase, converting the GTPase to its inactive form. Guanine nucleotide exchange factors mediate the exchange of GDP for GTP, turning the GTPase "on". Rabs are geranylgeranylated at one or two cysteine residues at their $\mathrm{C}$-termini and in this way can reversibly associate with their specific target membrane. Rab effectors are defined as molecules that only interact with a Rab protein when it is in its active, GTP-bound state (reviewed in Stenmark 2009).

$\operatorname{Atg} 16 \mathrm{~L}$ is an effector of the Golgi-resident Rab33B and to a lesser extent also Rab33A (Itoh 2008). Itoh et al. (2008) observed that Rab33B interacts with the Cterminal portion of the coiled coil domain of Atg16L1 (residues 141-265) and not the Nterminal portion of the coiled coil domain which mediates homo-oligomerization. They also concluded that Rab33B plays a regulatory role during autophagosome formation since the GTPase-deficient mutant (Q92L) attenuated basal autophagy despite induction of LC3I lipidation. In contrast, they observed that the constitutive-negative mutant (T47N) of Rab33B did not affect autophagy under nutrient-rich conditions. Furthermore, overexpression of the Rab33B-binding domain of Atg16L had an inhibitory effect on autophagy (Itoh 2008). Rab proteins interacting with coiled coil proteins have been observed before. Structures that were determined include Rab5-Rabaptin5, Rab6GCC185, Rab7-RILP, Rab11-FIP2, and Rab11-FIP3 (Zhu 2004, Burguete 2008, Wu 2005, Jagoe 2006, Eathiraj 2006).

\subsubsection{Atg16L and Crohn's disease}

Crohn's disease is an inflammatory bowel disease whereby chronic inflammation affects the gastrointestinal tract, usually the distal small intestine and colon (reviewed in Cho 2008). These regions have the highest concentration of bacteria and a disturbance in the regulation of the immune response to commensal bacteria is thought to stimulate inflammatory bowel disease. The epithelium usually serves as a barrier against bacteria and includes specialized cell types like Paneth cells which secrete antimicrobial peptides 
(Cho 2008). Several genome-wide association screens showed a direct link between Crohn's disease and a single nucleotide polymorphism in Atg16L1 that causes the T300A missense mutation in its WD40-repeat domain (Consortium 2007, Hampe 2007, Rioux 2007). Cadwell et al. (2010) showed that in mice, the phenotype of intestinal inflammation was caused by the interplay of the homozygous T300A mutation of Atg16L1 and infection with a virus since the uninfected Atg16L1 mutant animals did not exhibit Paneth cell pathogenesis. In addition, Kuballa et al. (2008) reported a link between the impaired capture of intracellular Salmonella typhimurium bacteria and the T300A mutation in Atg16L1 associated with Crohn's disease susceptibility.

The first identified susceptibility gene for Crohn's disease was a mutation in the NOD2 gene (Hugot 2001). Recently mutant Nod2 was shown to impair the autophagocytosis of invading bacteria through a reduction in the recruitment of Atg16L1 to the bacterial entry site in the plasma membrane (Travassos 2010).

Furthermore, Atg16L1 has been found to be important in the suppression of the intestinal inflammatory response as Atg16L1-deficient mice produce higher amounts of the inflammatory cytokines IL-1 $\beta$ and IL18 in response to stimulation with endotoxin (Saitoh 2008).

\subsection{Localization of Atg5 Atg16 to autophagosomal membranes}

\subsubsection{Autophagy and phosphoinositides}

PI(3)P has an essential role in autophagosome formation (Burman 2010). The membrane phospholipid phosphatidylinositol (PI) becomes phosphorylated at the 3' position of its inositol head group to form PI(3)P by the type III PI(3)K Vps34 complex I (Figure 1.8 panel A) (Schu 1993). Upon induction of autophagy, large amounts of PI(3)P are transported into the vacuole as a component of the autophagosome membrane, highlighting an enrichment of PI(3)P in autophagosomal membranes and its involvement in the formation of autophagosomes (Obara 2008). PI can also be phosphorylated at it 4' and 5' positions by different classes of PI kinases which can lead to double and triple phosphorylation products (Figure 1.8 panel B) (Vicinanza 2008). The phosphorylation of phosphatidylinositol-phosphates (PIPs) is reversible and this action is performed by specific phosphatases. This allows for tight regulation of membrane trafficking as PIP 
content contributes to individuality amongst membrane compartments as seen in panel C of Figure 1.8 (Vicinanza 2008).

Interestingly, it was shown by Ferguson et al. (2009) that $\mathrm{PI}(3,5) \mathrm{P}_{2}$ also plays a role in mammalian autophagy. Mutations which inhibit the conversion of $\mathrm{PI}(3) \mathrm{P}$ to $\mathrm{PI}(3,5) \mathrm{P}_{2}$ disrupt the degradation of cytoplasmic inclusion bodies via autophagy in the central nervous system of mice and cause neurodegeneration.

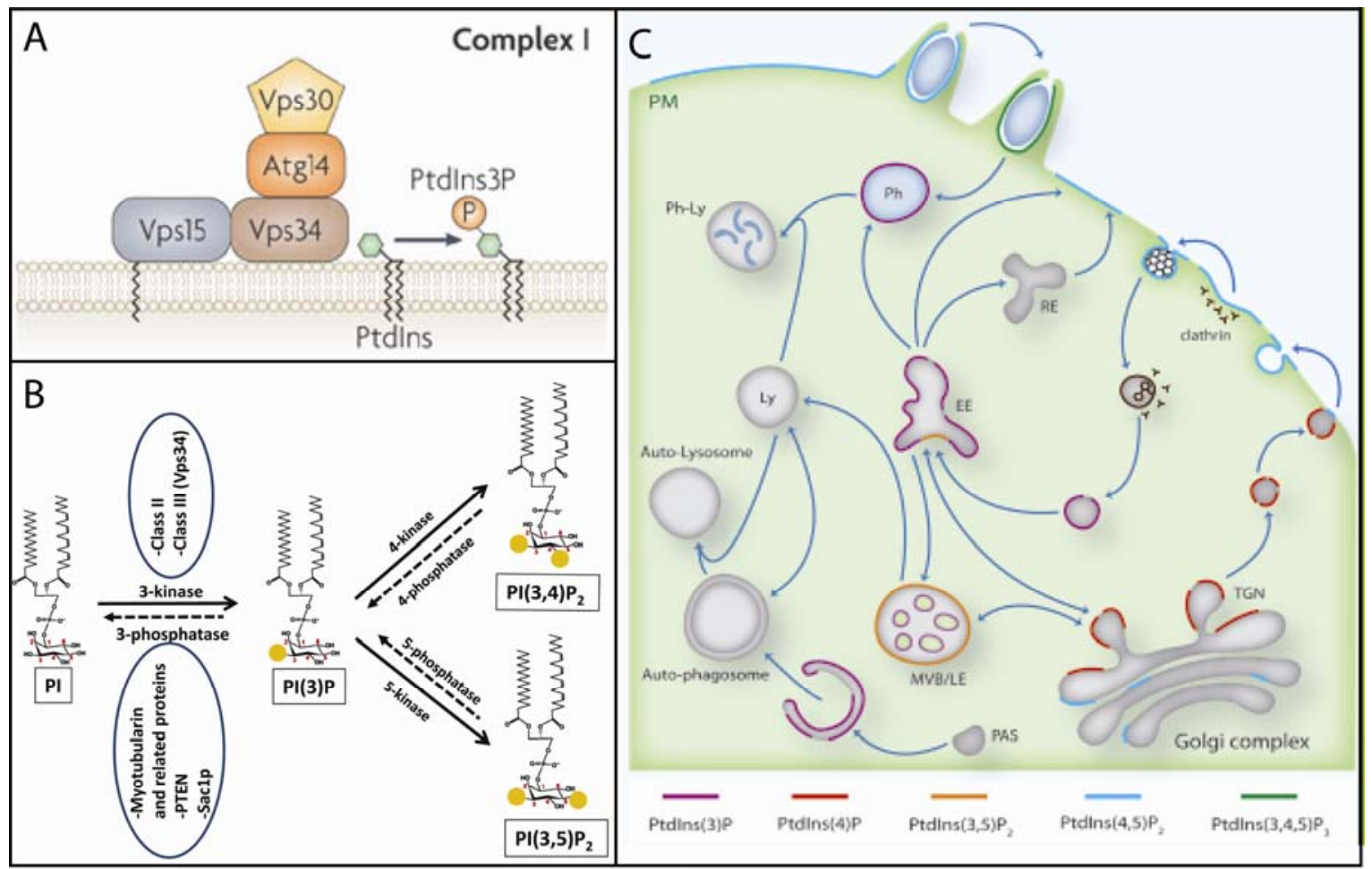

Figure 1.8: $\mathrm{PI}(3) \mathrm{P}$ is produced in autophagosomal membranes. (A) Production of $\mathrm{PI}(3) \mathrm{P}$ by the $\mathrm{PI}(3) \mathrm{K}$ complex I is essential for autophagy (from Nakatogawa 2009). (B) Further phosphorylation and dephosphorylation events of PI(3)P (from Burman 2010). (C) Abundance of specific PIPs in organellar membranes allows for compartment identity and regulation of membrane trafficking (from Vicinanza 2008).

\subsubsection{Requirements for isolation membrane recruitment of the Atg5-Atg16 complex}

Association of the Atg12-Atg5 Atg16L1 complex with the growing isolation membranes is essential for autophagosome formation (Suzuki 2001, Mizushima 2001). Suzuki et al. (2001) observed that in yeast the localization of GFP-Atg5 to the PAS depends on Atg16. Mizushima et al. (2003) discovered that localization of mammalian GFP-Atg16L was disrupted in Atg5-deficient ES cells and that Atg12 conjugation to Atg5 was not essential for recruitment of Atg16 by Atg5. Suzuki et al. (2007a) presented evidence that Atg5 recruitment to the PAS requires Atg16 and vice versa. Further evidence for the importance of an interaction between Atg5 and Atg16 for proper 
localization comes from the following experiment. When residues Arg35 and Phe46 of Atg16 which both interact with Atg5 are mutated, complex formation is disrupted and the Atg5 Atg16 complex no longer localizes at the PAS (Matsushita 2007).

Localization of the Atg5 Atg16 complex also requires PI(3)P. Deleting either Atg14 or Vps30, both components of the yeast PI(3)K complex I, inhibits binding of the Atg12-Atg5 Atg16 complex to the isolation membrane (Suzuki 2007a). Further evidence comes from the observation that inhibiting PI(3)K activity with wortmannin and 3methyladenine impairs recruitment of Atg5 to punctuate pre-autophagosomal structures (Mizushima 2001).

Certain classes of proteins require the ability to bind specific phosphoinositides to mediate their functions in membrane trafficking: small GTPase accessory proteins; coats and adaptor proteins; sorting nexins and ESCRT proteins; fission and fusion proteins; and actin and microtubule-based motor proteins (reviewed in Vicinanza 2008). These proteins contain lipid binding domains which confer their phosphoinositide specificities. For example, proteins which contain a zinc finger FYVE (Fab1, YOTB, Vac1, EEA1) domain bind PI(3)P (reviewed in Lemmon 2008). PX (Phox homology) domains adopt an $\alpha / \beta$ structure and interact with PI(3)P and several other phosphoinositides. PROPPIN ( $\beta$ propeller that binds phosphoinositides) domains have a $\beta$-propeller fold and bind $\mathrm{PI}(3,5) \mathrm{P}_{2}$ and PI(3)P. BAR (Bin, Amphiphysin, Rvs) domains are extended $\alpha$-helical bundles which associate with acidic phospholipids (Lemmon 2008). Structures of PIP binding proteins are shown in Figure 1.9.

The PIP effectors involved in autophagy typically contain canonical PI(3)P lipid binding motifs. Alfy and DFCP1 have one or two FYVE domains, respectively (Simonsen 2004, Ridley 2001). Of note, early endosomal autoantigen 1 (EEA1) is targeted to endosomes via its FYVE domain, and increased aviditiy through dimerization via its coiled coil domain is required for efficient membrane targeting (Gillooly 2000, Dumas 2001, Hayakawa 2004). Atg20 and Atg24 are involved in the selective autophagy Cvt pathway and contain PX domains (Nice 2002). Atg18, Atg21, and Ygr223 are WD40repeat proteins which preferentially bind $\mathrm{PI}(3) \mathrm{P}$ and $\mathrm{PI}(3,5) \mathrm{P}_{2}$ via their conserved FRRGT motif (Barth 2001, Guan 2001, Barth 2002, Meiling-Wesse 2004, Dove 2004, Stromhaug 2004, Krick 2008b). 


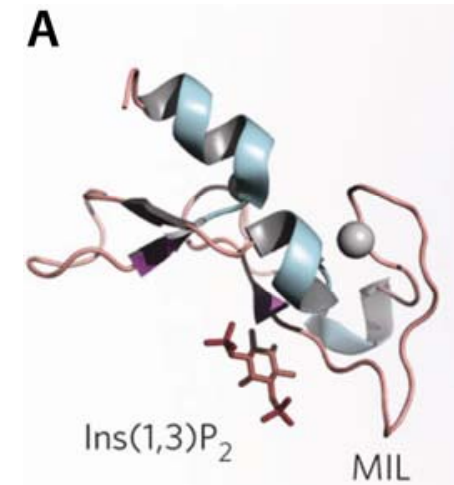

EEA1 FYVE
B

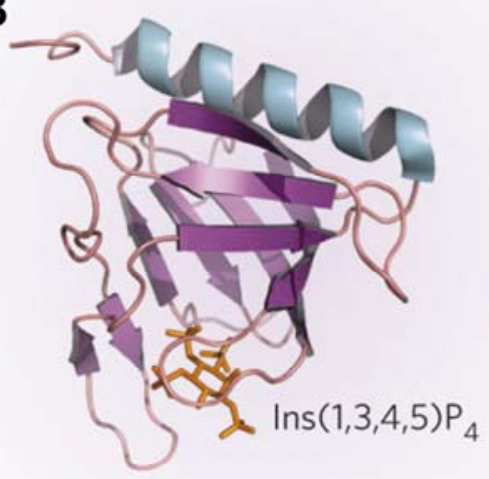

Grp1 PH

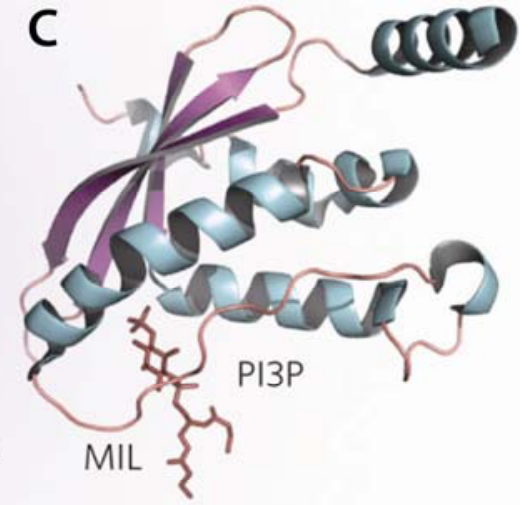

P40 phox PX

Figure 1.9: Crystal structures of phosphoinositide binding domains bound to their specific phosphoinositide or soluble headgroup. (A) The FYVE domain of EEA1 in complex with inositol 1,3bisphosphate $\left(\operatorname{Ins}(1,3) \mathrm{P}_{2}\right)$, the soluble headgroup moiety of Ptdlns(3)P (PDB ID: 1JOC, Dumas 2001), (B) the Grp1 PH domain in complex with inositol 1,3,4,5-tetrakisphosphate (Ins $\left.(1,3,4,5) \mathrm{P}_{4}\right),(P D B$ ID: 1FGY, Lietzke 2000) and (C) the p40 phox PX domain in complex with dibutanoyl PI(3)P (PDB ID: $1 \mathrm{H} 6 \mathrm{H}$, Bravo 2001).

Since Atg5 and Atg16 both lack canonical lipid binding motifs, it is possible that the complex binds to the membrane either through an unidentified adaptor protein or directly with a novel lipid binding motif. The probable mechanism of Atg5 Atg16 complex recruitment to the autophagosomal membrane has, until now, remained unanswered.

\subsection{Aims of This Work}

It was the objective of this study to characterize the mammalian Atg5 Atg16L1 complex essential for expansion of the isolation membrane during autophagosome formation. At the time this study was begun in October 2007 it was known that the coiled coil domain of Atg16 mediates oligomerization of the whole Atg12-Atg5 Atg16 complex. It was shown by analytical gel filtration that a $\sim 350 \mathrm{kDa}$ multimer was present in yeast which would correspond to a tetramer whereas the murine complex formed an $\sim 800 \mathrm{kDa}$ complex, likely an octameric complex (Kuma 2002, Mizushima 2003). To resolve these discrepancies, multiple angle laser light scattering was used to accurately determine the molecular mass and stoichiometry of the mammalian Atg5 Atg16L1 complex.

One of my goals was to use x-ray crystallography to determine the structure of an oligomerized Atg5 Atg16L1 complex since multimerization of the Atg12-Atg5 Atg16 complex is required for expansion of the isolation membrane. I therefore aimed to identify a minimal oligomerized Atg5 Atg16L1 complex for crystallization trials. A series of 
truncated Atg16L1 constructs were purified and their oligomerization states were characterized. Right before this study started, Matsushita et al. (2007) published the structure of yeast Atg5 in complex with the N-terminal Atg5-binding domain of Atg16. I also set out to crystallize the corresponding non-oligomerized, minimal mammalian Atg5 Atg16L1(1-70) complex to compare it with the published yeast structure.

Another important objective of this project was the characterization of interactions of Atg5 and Atg16L1 with other binding partners. For example, one binding partner of Atg16L is the GTPase Rab33B (Itoh 2008). I further characterized the Rab33B-binding site of Atg16L1. Following up on the studies initiated by our lab rotation student Adi Bar Shalom, I also investigated the cleavage of the Atg5 Atg16L1 complex by the protease calpain.

Since the Atg5 Atg16 complex associates with the growing isolation membrane, I tested for lipid binding of the complex and showed that the Atg5 Atg16L1 complex directly interacts with phosphoinositides. The effect of the coiled coil domain of Atg16L1 on PIP binding was also elucidated. Co-crystallization and soaking of both yeast and mammalian Atg5 Atg16(L1) complexes with the inositol head group moiety of PI(3)P or $\mathrm{PI}(5) \mathrm{P}$ was also performed to illuminate the exact binding mechanism of Atg5 Atg16 to the headgroup of this lipid, thus helping to understand recruitment of this peripheral membrane protein complex to the growing isolation membranes. 


\section{MATERIALS AND METHODS}

\subsection{Molecular biology techniques}

\subsubsection{Cloning \& mutagenesis}

\section{PCR to create murine Atg5, Atg16L1 and Rab33B Q92L constructs}

The chosen cDNA fragments were amplified in vitro using the polymerase chain reaction (PCR) (Saiki 1988, Higuchi 1988). The double-stranded template DNA was initially denatured at $95^{\circ} \mathrm{C}$ for 1 minute $(\mathrm{min})$ to produce single-stranded DNA. Each of the 25 amplification cycles began with denaturation of the DNA at $95{ }^{\circ} \mathrm{C}$ for 30 seconds. The temperature was then reduced to $48{ }^{\circ} \mathrm{C}$ for 45 seconds which allowed annealing of the two primers to the 5' and 3' ends of the cDNA. This was followed by a 2 min elongation step at $70{ }^{\circ} \mathrm{C}$ where the thermostable polymerase synthesized the DNA strands complementary to the cDNA by adding the matching dNTPs to the primers. A final elongation step at $70{ }^{\circ} \mathrm{C}$ for 10 min completed the amplification process. The tubes were kept cool at $4{ }^{\circ} \mathrm{C}$ until they were taken out. PCR amplifications were performed with a Thermo PCR Sprint Thermal Cycler (Thermo Electron Corporation) with a heating block fitting $240.2 \mathrm{ml}$ tubes. Final concentrations of the components for a PCR reaction were as follows: $0.2 \mathrm{mM}$ dNTPs, $2 \mathrm{pmol} / \mu \mathrm{L}$ forward primer, $2 \mathrm{pmol} / \mu \mathrm{L}$ reverse primer, $1 \mathrm{x}$ polymerase buffer containing $\mathrm{MgCl}_{2} \cdot 6 \mathrm{H}_{2} \mathrm{O}, 0.02-0.1 \mathrm{ng} / \mu \mathrm{L}$ cDNA template, and Expand High Fidelity DNA polymerase (Roche) with an activity of $7 \mathrm{U}$ per $150 \mu$ reaction mix.

The following constructs were prepared for this study (Table 2.1). Forward and reverse primers and the corresponding restriction sites are listed in Table 2.2. The cDNA clones given in Table 2.3 were used as templates during PCR. The murine Atg16L1 cDNA encoded the $\gamma$ isoform, which includes all of the exons and is the isoform found primarily in the brain.

Full-length mRab33B(1-229)Q92L and truncated mRab33B(30-202)Q92L containing the Q92L point mutation were amplified by two-step PCR. In the first step, the following constructs were made using forward and reverse primers which both contained the respective mutation (Table 2.2): mRab33B(1-95)Q92L and mRab33B(30-95)Q92L were generated for use as forward templates and mRab33B(89-229)Q92L and $\mathrm{mRab} 33 \mathrm{~B}(89-202)$ as reverse templates for the second PCR reaction. 
Table 2.1: Constructs utilized in this study

\begin{tabular}{|c|c|c|c|c|c|c|}
\hline & \multicolumn{2}{|c|}{ MCS1 } & MCS1 & MCS2 & MCS2 & \\
\hline Plasmid & \multicolumn{2}{|c|}{ Construct } & $\begin{array}{l}\text { Restriction } \\
\text { Sites }\end{array}$ & Construct & $\begin{array}{l}\text { Restriction } \\
\text { Sites }\end{array}$ & Source \\
\hline pJET1 & \multicolumn{2}{|l|}{ mAtg16L1(1-70) } & NdeI/NotI & $\mathrm{n} / \mathrm{a}$ & ------------ & Schalk \\
\hline pET-28a & \multicolumn{2}{|l|}{ mAtg16L1(1-70) } & NdeI/NotI & $\mathrm{n} / \mathrm{a}$ & ------------- & Schalk \\
\hline pET-28a & \multicolumn{2}{|l|}{ mAtg16L1(1-231) } & NdeI/NotI & $\mathrm{n} / \mathrm{a}$ & ------------ & Kühnel \\
\hline pGEX4T1 & \multicolumn{2}{|l|}{ mAtg16L1(328-623) } & XhoI/NotI & $\mathrm{n} / \mathrm{a}$ & ------------- & Schalk \\
\hline pJET1 & \multicolumn{2}{|l|}{ mAtg16L1(1-623) } & NdeI/NotI & $\mathrm{n} / \mathrm{a}$ & ------------ & Schalk \\
\hline pET-28a & \multicolumn{2}{|l|}{ mAtg16L1(1-623) } & NdeI/NotI & $\mathrm{n} / \mathrm{a}$ & ------------- & Schalk \\
\hline pETDuet1 & \multicolumn{2}{|l|}{$h A \operatorname{tg} 5(1-275)$} & BamHI/NotI & empty & ------------ & Kühnel \\
\hline pET-28a & \multicolumn{2}{|l|}{ mAtg16L1(1-106) } & NdeI/NotI & $\mathrm{n} / \mathrm{a}$ & |---------- & Schalk \\
\hline pET-28a & \multicolumn{2}{|l|}{$m A \operatorname{tg} 16 \mathrm{~L} 1(1-113)$} & NdeI/NotI & $\mathrm{n} / \mathrm{a}$ & ------------ & Schalk \\
\hline pET-28a & \multicolumn{2}{|l|}{ mAtg16L1(1-155) } & NdeI/NotI & $\mathrm{n} / \mathrm{a}$ & |----------- & Schalk \\
\hline pET-28a & \multicolumn{2}{|l|}{ mAtg16L1(1-168) } & NdeI/NotI & $\mathrm{n} / \mathrm{a}$ & ------------ & Schalk \\
\hline pET-28a & \multicolumn{2}{|l|}{ mAtg16L1(24-168) } & NdeI/NotI & $\mathrm{n} / \mathrm{a}$ & ------------ & Schalk \\
\hline pET-28a & \multicolumn{2}{|l|}{ mAtg16L1(53-168) } & NdeI/NotI & $\mathrm{n} / \mathrm{a}$ & ------------- & Schalk \\
\hline pET-28a & \multicolumn{2}{|l|}{ mAtg16L1(1-265) } & NdeI/NotI & $\mathrm{n} / \mathrm{a}$ & ----------- & Schalk \\
\hline pET-28a & \multicolumn{2}{|c|}{ mAtg16L1(1-265) K14A } & NdeI/NotI & $\mathrm{n} / \mathrm{a}$ & ------------ & Schalk \\
\hline $\mathrm{pET}-28 \mathrm{a}$ & \multicolumn{2}{|c|}{ mAtg16L1(1-265) R15A } & NdeI/NotI & $\mathrm{n} / \mathrm{a}$ & ------------ & Schalk \\
\hline pET-28a & \multicolumn{2}{|c|}{$\mathrm{mAtg} 16 \mathrm{~L} 1(1-265) \mathrm{R} 22 \mathrm{~A}$} & NdeI/NotI & $\mathrm{n} / \mathrm{a}$ & ------------ & Schalk \\
\hline pET-28a & \multicolumn{2}{|c|}{ mAtg16L1(1-265) K14AR15A } & NdeI/NotI & $\mathrm{n} / \mathrm{a}$ & ------------ & Schalk \\
\hline pET-28a & \multicolumn{2}{|c|}{ mAtg16L1(1-265) K14AR15AR22A } & NdeI/NotI & $\mathrm{n} / \mathrm{a}$ & ------------ & Schalk \\
\hline pETDuet1 & \multicolumn{2}{|c|}{ mAtg5(1-275) +Thr. site } & SalI/NotI & empty & ------------- & Schalk \\
\hline pGEX4T1 & \multicolumn{2}{|c|}{ mRab33B(1-229) Q92L+Thr. site } & BamHI/NotI & empty & ------------- & Schalk \\
\hline pETDuet1 & \multicolumn{2}{|c|}{ mRab33B(30-202) Q92L+Thr. site } & BamHI/NotI & empty & ------------ & Schalk \\
\hline pETDuet1 & $\begin{array}{l}\text { mRab33B(30-202) } \\
\text { Q92L+Thr. site }\end{array}$ & BamHI/NotI & m Atg16L & 141-265) & NdeI/KpnI & Schalk \\
\hline pETDuet1 & $\begin{array}{l}\text { mRab33B(30-202) } \\
\text { Q92L+Thr. site }\end{array}$ & BamHI/NotI & $\mathrm{m} \operatorname{Atg} 16 \mathrm{~L}$ & $(172-265)$ & NdeI/KpnI & Schalk \\
\hline pETDuet1 & $\begin{array}{l}\text { mRab33B(30-202) } \\
\text { Q92L+Thr. site }\end{array}$ & BamHI/NotI & $\mathrm{m} \operatorname{Atg} 16 \mathrm{~L}$ & 172-234) & NdeI/KpnI & Schalk \\
\hline pETDuet1 & $\begin{array}{l}\text { mRab33B(30-202) } \\
\text { Q92L+Thr. site }\end{array}$ & BamHI/NotI & $\mathrm{m} \operatorname{Atg} 16 \mathrm{~L}$ & 203-265) & NdeI/KpnI & Schalk \\
\hline pETDuet1 & $\begin{array}{l}\text { mRab33B(30-202) } \\
\text { Q92L+Thr. site }\end{array}$ & BamHI/NotI & m Atg16L & $(125-204)$ & NdeI/KpnI & Schalk \\
\hline pETDuet1 & $y \operatorname{Atg} 5(1-294)$ & BamHI/NotI & $\mathrm{yAtg} 16$ & $-46)$ & NdeI/XhoI & Morales \\
\hline pETDuet1 & yAtg5(1-294) & BamHI/NotI & $\mathrm{y} \operatorname{Atg} 1$ & $1-57)$ & NdeI/XhoI & Morales \\
\hline pETDuet1 & yAtg5(1-294) & BamHI/NotI & y Atg1 & $-67)$ & NdeI/XhoI & Hellwig \\
\hline pETDuet1 & yAtg5(1-294) & BamHI/NotI & y $\operatorname{Atg} 16(1$ & 67) R9A & NdeI/XhoI & Hellwig \\
\hline pETDuet1 & $y \operatorname{Atg} 5(1-294)$ & BamHI/NotI & y $\operatorname{Atg} 16(1$ & 7) $\mathrm{K} 10 \mathrm{~A}$ & NdeI/XhoI & Hellwig \\
\hline pETDuet1 & yAtg5(1-294) & BamHI/NotI & $y \operatorname{Atg} 16(1$ & 7) $\mathrm{K} 11 \mathrm{~A}$ & NdeI/XhoI & Hellwig \\
\hline pETDuet1 & yAtg5(1-294) & BamHI/NotI & y $\operatorname{Atg} 16(1-6$ & R9AK10A & NdeI/XhoI & Hellwig \\
\hline pETDuet1 & yAtg5(1-294) & BamHI/NotI & y $\operatorname{Atg} 16(1-6$ & R9AK11A & NdeI/XhoI & Hellwig \\
\hline pETDuet1 & $y \operatorname{Atg} 5(1-294)$ & BamHI/NotI & $\begin{array}{r}\text { yAtg1c } \\
\text { K10A }\end{array}$ & $\begin{array}{l}1-67) \\
11 \mathrm{~A} \\
\end{array}$ & NdeI/XhoI & Hellwig \\
\hline pETDuet1 & $y \operatorname{Atg} 5(1-294)$ & BamHI/NotI & $\begin{array}{r}\text { y Atg1 } \\
\text { R9AK10 }\end{array}$ & $\begin{array}{l}(1-67) \\
\mathrm{AK} 11 \mathrm{~A} \\
\end{array}$ & NdeI/XhoI & Hellwig \\
\hline
\end{tabular}

MCS=multiple cloning site, $\mathrm{h}=$ human, $\mathrm{m}=$ murine, $\mathrm{y}=$ yeast, $\mathrm{Thr}=$ thrombin 
Table 2.2: Primer list

\begin{tabular}{|c|c|c|}
\hline Primer Name & Sequence $\left(5^{\prime}\right.$, to $\left.3^{\prime}\right)$ & Company \\
\hline mAtg16L1_NdeI_1f & 5'-ggaattccatatgtcgtcgggcctgcgcg-3' & MWG-Biotech \\
\hline mAtg16L1_NotI_70rv & 5'-ataagaatgcggccgctcaacttatttcatgcctatttggcat-3' & MWG-Biotech \\
\hline mAtg16L1_NotI_231rv & 5'-gatagcggccgctcatagaggttcctttgctgcttctg-3' & MWG-Biotech \\
\hline mAtg16L1_NotI_623rv & 5'-gatagcggccgctcaaggctgtgcccacagcac-3' & MWG-Biotech \\
\hline mAtg16L1 XhoI 328f & 5'-ccgctcgagactactgcctcotatgtcttcg-3, & MWG-Biotech \\
\hline mAtg16L1_NotI_106rv & 5'-aaggaaaaaagcggccgctcataactccccacgcttcttgtg-3' & Sigma-Genosys \\
\hline mAtg16L1_NotI 113rv & 5'-aaggaaaaaagcggccgctcacaggtcaatcaccaactgagc-3' & Sigma-Genosys \\
\hline mAtg16L1_NotI_155rv & 5'-aaggaaaaaagcggccgctcagaggtcctgcagtttgtgc-3' & Sigma-Genosys \\
\hline mAtg16L1_NotI_168rv & 5'-aaggaaaaaagcggccgctcaggcgtcatactcatcctt cag-3' & Sigma-Genosys \\
\hline mAtg16L1 NotI 265rv & 5'-aaggaaaaaagcggccgctcaagtagctgctctgctga cagc-3, & Sigma-Genosys \\
\hline mAtg16L1_NdeI_24f & 5'-gggaattccatatgcgggaccgactgcagaggc-3' & Sigma-Genosys \\
\hline mAtg16L1_NdeI_53f & 5'-gggaattccatatgacccagaaactacaagcagaaaa-3' & Sigma-Genosys \\
\hline mAtg5 Thrombin_SalI_1f & $\begin{array}{l}\text { 5'-gtaacgcgtcgacctggtgccgcgcggcagcatgacagatga } \\
\text { caaagatgtgct-3' }\end{array}$ & Sigma-Genosys \\
\hline mAtg5_NotI_275rv & 5'-aaggaaaaaagcggccgctcaatctgttggctgggggac-3' & Sigma-Genosys \\
\hline mRab33B_Thrombin_BamHI_1f & cgcggatccgctggtgccgcgcggcagcatgacttcggagatggagtcg & Sigma-Genosys \\
\hline mRab33B_Thrombin_BamHI_30f & cgcggatccgetggtgccgegcggcagccgctcccgcatcttcaagatc & Sigma-Genosys \\
\hline mRab33B_NotI_202rv & 5'-aaggaaaaaagcggccgctcaagtagctgctctgctgacagc-3' & Sigma-Genosys \\
\hline mRab33B_NotI_229rv & 5'-aaggaaaaaagcggccgctcagcaccagcaagtcaccg-3' & Sigma-Genosys \\
\hline mRab33B_Q92L_fw & 5'-cacggcagggctggagcggttca-3' & Sigma-Genosys \\
\hline mRab33B_Q92L_rv & 5'-tgaaccgctccagccetgccgtg-3' & Sigma-Genosys \\
\hline mAtg16L1 NdeI $125 \mathrm{f}$ & 5'-ggaattccatatgcagatgaatgaagcaaagatttcg-3' & Sigma-Genosys \\
\hline mAtg16L1_NdeI_141f & 5'-ggaattccatatgctggagacaaactgcctggac-3' & Sigma-Genosys \\
\hline mAtg16L1_NdeI_172f & 5'-ggaattccatatgacttttactgccctagaagagaa-3' & Sigma-Genosys \\
\hline mAtg16L1_NdeI_203f & 5'-ggaattccatatgaatcgcctcaatgcagagaatg-3', & Sigma-Genosys \\
\hline mAtg16L1_KpnI_204rv & 5'-cggggtactcagcgattggcttcttgggcctt-3' & Sigma-Genosys \\
\hline mAtg16L1_KpnI_234rv & 5'-cggggtacctcaaacaggtagaggttcctttgct-3' & Sigma-Genosys \\
\hline mAtg16L1_KpnI_265rv & 5'-cggggtacctcaagtagctgctctgctgacagc-3' & Sigma-Genosys \\
\hline mAtg16L1_K14A_fw & 5'-actttccccgctgggcgcgtcacatcgcgg-3' & Sigma-Genosys \\
\hline mAtg16L1_R15A_fw & 5'-tcccegctggaaggctcacatcgcggag-3' & Sigma-Genosys \\
\hline mAtg16L1 R22A fw & 5'-gcggaggaactggcgcgccgggaccg-3' & Sigma-Genosys \\
\hline mAtg16L1_K14AR15A_fw & 5'-cagactttccccgctgggcggctcacatcgcggaggaac-3' & Sigma-Genosys \\
\hline mAtg16L1_K14A_rv & 5'-ccgcgatgtgacgcgcccagcggggaaagt-3' & Sigma-Genosys \\
\hline mAtg16L1_R15A_rv & 5'-ctccgcgatgtgagccttccagcgggga-3' & Sigma-Genosys \\
\hline mAtg16L1_R22A_rv & 5'-cggtcceggcgcgccagttcctccgc-3' & Sigma-Genosys \\
\hline mAtg16L1_K14AR15A_rv & 5'-actttcccegctgggcgegtcacatcgcgg-3' & Sigma-Genosys \\
\hline hAtg5 BamHI $1 \mathrm{fw}$ & 5'-cgggatccgatgacagatgacaaagatgtgct-3' & MWG-Biotech \\
\hline hAtg5_NotI_275rv & 5'-ataagaatgcggccgctcaatctgttggctgtgggatg $-3^{\prime}$ & MWG-Biotech \\
\hline yAtg16 NdeI_1fw & 5'-gggaattccatatgggcaactttattatcacggag-3' & Sigma-Genosys \\
\hline yAtg16 XhoI 46rv & 5'-caccgctcgagtcaaaacagttcgttcaggtgtgcctc-3' & Sigma-Genosys \\
\hline yAtg16_XhoI_57rv & 5'-caccgctcgagtcaaatgttgccaccaatggcaccgc-3' & Sigma-Genosys \\
\hline yAtg16 XhoI $67 \mathrm{rv}$ & 5'-caccgctcgagtcaggtattcagcagagcatcatcgtg-3' & Sigma-Genosys \\
\hline
\end{tabular}

$\mathrm{h}=$ human, $\mathrm{m}=$ murine, $\mathrm{y}=$ yeast 
Table 2.3: cDNA clones used as PCR templates

\begin{tabular}{|c|c|c|c|c|}
\hline Organism & Gene & Plasmid & ID & Company \\
\hline Homo sapiens & Atg5 & & & Biocat \\
\hline Mus musculus & Atg5 & pCMV-SPORT6 & BC002166 & Biocat \\
\hline Mus musculus & Atg16L1 isoform 3 & pYX-ASc & BC049122 & Biocat \\
\hline Mus musculus & Rab33B & pYX-ASc & BC065076 & Biocat \\
\hline Saccharomyces cerevisiae & Atg5 & pMA & 0953294 & Mr. Gene \\
\hline Saccharomyces cerevisiae & Atg16 & pMA & 0953293 & Mr. Gene \\
\hline
\end{tabular}

\section{DNA agarose gel electrophoresis}

DNA of different sizes was visualized by agarose gel electrophoresis. $100 \mathrm{mM}$ TBE buffer (50 mM Tris, $50 \mathrm{mM} \mathrm{H} \mathrm{BO}_{3}, 0.1 \mathrm{mM}$ EDTA) was poured over a flatbed agarose gel (1\% agarose, $100 \mathrm{mM}$ TBE buffer) in a self-made gel chamber. Samples were combined with 6x DNA loading dye (Fermentas) and loaded into the wells. Upon application of $100 \mathrm{~V}$ to the chamber, the DNA migrated toward the positive electrode on the opposite end of the chamber due to the negatively charged phosphate groups that comprise part of the DNA backbone. Smaller DNA fragments have a higher mobility through the agarose gel matrix than larger fragments. Visualization of DNA was accomplished using GelGreen ${ }^{\mathrm{TM}}$ Nucleic Acid Gel Stain (Biotium) which has a high affinity for double-stranded DNA, absorbs UV light between 250 and $300 \mathrm{~nm}$, and emits fluorescent light at $\sim 550 \mathrm{~nm}$. This dye is more stable, sensitive, and a safer alternative to the previously utilized DNA intercalator, ethidium bromide (Waring 1965). Sizes of DNA fragments were estimated using either the Generuler $1 \mathrm{~kb}$ or the GeneRuler $100 \mathrm{bp}$ Plus ladder (Fermentas) as a marker.

\section{Gel extraction purification}

Resolved PCR products and DNA digested with restriction enzymes were extracted from the gel for purification using a NucleoSpin ${ }^{\circledR}$ Extract II PCR cleanup/gel extraction kit (Macherey Nagel) and tabletop centrifuge. The extracted, weighed gel fragment was combined with Binding Buffer NT and heated to melt the gel. The DNA was bound to the silica membrane on a mini spin column due to the presence of chaotropic salt in the binding buffer. Contaminations are removed in a wash step with ethanol-containing Wash Buffer NT3. The DNA is eluted from the membrane under low ionic strength conditions with $50 \mu \mathrm{L} 5 \mathrm{mM}$ Tris $/ \mathrm{HCl} \mathrm{pH} 8.5$ buffer that was preheated to $70^{\circ} \mathrm{C}$. 


\section{Restriction enzyme digestion}

The primers given in Table 2.2 introduced restriction sites at the 5' and 3' end of the amplified PCR. Both the gel purified PCR product $(\sim 20 \mu \mathrm{L})$ and the plasmid $(\sim 1-2 \mu \mathrm{g})$ were digested with $0.5-2 \mathrm{U} / \mu \mathrm{L}$ final concentration of the designated restriction enzymes (New England Biolabs) for 1 to 3 hours at $37^{\circ} \mathrm{C}$ in the corresponding digestion buffer with the occasional addition of BSA depending upon the requirements of the enzymes.

\section{Ligation reactions}

After digestion and purification, $100 \mathrm{ng}$ of the plasmid was combined with 10 to 15-fold molar excess of the insert, the amount of which was calculated using the following equation: [Weight of plasmid x (bp of insert) $\mathrm{x}$ molar ratio]/(bp of plasmid)]. The components were combined with 1x ligase buffer (New Engliand Biolabs) and $60 \mathrm{U} / \mu \mathrm{L}$ bacteriophage T4 DNA ligase (New Engliand Biolabs). The reaction was incubated for 30 to $120 \mathrm{~min}$ at room temperature (RT).

Since ligation of a digested PCR product directly into a vector can be difficult, the GeneJET PCR Cloning Kit (Fermentas) was utilized in some cases. The pJET1.2 plasmid (Fermentas) encodes a restriction endonuclease that is lethal to the transformed cell if it is not interrupted by insertion of a PCR product. In the Sticky-End protocol, any 3'-dA overhangs are cut by a thermostable DNA blunting enzyme, which could have been formed by the Taq polymerase present in the Expand High Fidelity DNA polymerase mix. The blunt end constructs were then ligated into the pJET1.2/blunt cloning vector using T4 DNA Ligase (Fermentas).

\section{Multi site-directed mutagenesis}

The QuikChange Lightning Muli Site-Directed Mutagenesis Kit (Agilent Technologies) was utilized. The protocol was followed according to the instruction manual. 100 ng of Atg16L1(1-265)pET28a plasmid DNA was used as a template during the PCR. The amount of mutagenic primer used was calculated according to the formula given in the instruction manual. Mutagenic primers (Table 2.2) only going in one direction were used per reaction. $0.75 \mu \mathrm{L}$ QuikSolution and $1 \mu \mathrm{L}$ dNTPs aliquotted from the kit were used per $25 \mu \mathrm{L}$ total reaction. During the PCR, the double-stranded plasmid DNA was denatured at $95{ }^{\circ} \mathrm{C}$ for 2 min. 25 cycles of the following steps were repeated: denaturing of the DNA at $95{ }^{\circ} \mathrm{C}$ for 20 seconds, the mutagenic primers were annealed to the DNA corresponding to the correct direction at $55{ }^{\circ} \mathrm{C}$ for 20 seconds, and the Pfu 
Fusion-based enzyme synthesized the DNA extending from the primers at $65{ }^{\circ} \mathrm{C}$ for 3 min. A final elongation step at $65{ }^{\circ} \mathrm{C}$ for $5 \mathrm{~min}$ was done. After the DNA with the mutation in one strand was amplified, the methylated template DNA was digested with 1 $\mu \mathrm{L}$ of the DpnI enzyme (provided in the kit) for $5 \mathrm{~min}$ at $37^{\circ} \mathrm{C} .1 .5 \mu \mathrm{L}$ of the mutated, single-stranded DNA was then transformed into $47 \mu \mathrm{L}$ chemically competent XL10 Gold ultracompetent cells (containing $2 \mu \mathrm{L} \beta$-mercaptoethanol provided in the kit) by heat shock at $42{ }^{\circ} \mathrm{C}$ for 30 seconds. $0.5 \mathrm{~mL}$ preheated NYZ+ broth was added to the cells and they were shaken at $250 \mathrm{rpm}$ for $1 \mathrm{hr}$ at $37{ }^{\circ} \mathrm{C}$ before being plated on LB agar plates containing the appropriate antibiotic for plasmid selection.

\subsubsection{Transformation into competent $\mathbf{E}$. coli cells}

\section{Heat shock transformation into chemically competent $E$. coli cells}

Chemically competent Escherichia coli cells (strains: XL1Blue, BL21 (DE3), or Rosetta 2 (DE3)) were thawed on ice and 10 ng of DNA was added. The cells were further chilled on ice for $30 \mathrm{~min}$. The cells were then heat shocked at $42{ }^{\circ} \mathrm{C}$ for 1 minute, causing the DNA to enter the competent cells (Mandel 1970, Dagert 1979). After cooling the cells on ice for 1 minute, $800 \mu \mathrm{L}$ of Luria Bertani (LB) media (1\% tryptone, $0.5 \%$ yeast extract, $1 \% \mathrm{NaCl}$ ) was added to the bacteria. Cells were shaken at 800 to $1000 \mathrm{rpm}$ at $37{ }^{\circ} \mathrm{C}$ for 60 min (Eppendorf Thermomixer Compact) to allow expression of proteins conferring antibiotic resistance. The cells were then gently spun down at $4000 \mathrm{rpm}$ in a tabletop centrifuge, and the majority of the supernatant was removed. The bacteria were resuspended in the remaining supernatant $(\sim 300 \mu \mathrm{L})$ and plated onto LB plates. pJET1.2, pETDuet-1, and pGEX-4T-1 plasmids were selected on LB plates containing $100 \mu \mathrm{g} / \mathrm{mL}$ ampicillin. The pET-28a plasmid was selected on LB plates containing $30 \mu \mathrm{g} / \mathrm{mL}$ kanamycin. In addition, Rosetta 2 (DE3) cells required supplementation with $35 \mu \mathrm{g} / \mathrm{mL}$ chloramphenicol to select the plasmid encoding rare tRNAs. The plates were incubated at $37^{\circ} \mathrm{C}$ overnight.

\section{Electroporation transformation into electrocompetent $\mathrm{E}$. coli cells}

DNA was transformed into electrocompetent $E$. coli cells according to the method developed by Dower et al. (1988). The cells were thawed on ice and 5 ng of DNA was transferred to the electrocompetent which were then chilled on ice for $20 \mathrm{~min}$ before being added to pre-cooled electroporation cuvettes. Cells were incubated in the pre-cooled cuvette for 1 minute before being electroporated with the Biorad Gene Pulser II (voltage 
of $2.1 \mathrm{kV}, 25 \mu \mathrm{F}$ capacitance, resistance $200 \Omega$ and a time constant of $5 \mathrm{~ms}$ ). The cells were then combined with $1 \mathrm{~mL} \mathrm{LB}$ medium and shaken at 800 to $1000 \mathrm{rpm}$ at $37^{\circ} \mathrm{C}$ for $\sim 60$ min using the Eppendorf Thermomixer Compact. The same remaining procedure as for the heat shock transformation protocol was followed.

\subsubsection{Plasmid isolation}

\section{Small scale purification}

Smaller quantities of plasmids "miniprep" were isolated from $5 \mathrm{~mL}$ E. coli cultures using the centrifugation protocol of the NucleoSpin ${ }^{\circledR}$ Plasmid (Machery Nagel). Cells were pelleted, resuspended in RNase containing buffer, and then lysed with alkaline/SDS buffer. The reaction was neutralized with buffer containing guanidine hydrochloride, a chaotropic salt, and the genomic DNA, cellular debris, and precipated protein were pelleted by centrifugation for $10 \mathrm{~min}$ at $13000 \mathrm{rpm}$. The cleared lysate was loaded onto the silica-based resin of the anion-exchange column and first washed with buffer containing guanidine hydrochloride and isopropanol preheated to $50{ }^{\circ} \mathrm{C}$, followed by washing with buffer containing isopropanol. The membrane was dried by centrifugation for 3 min before the DNA was eluted with $40 \mu$ of low ionic strength $5 \mathrm{mM}$ Tris/ $\mathrm{HCl} \mathrm{pH}$ 8.5 buffer preheated to $70{ }^{\circ} \mathrm{C}$. The purified plasmid DNA was and stored at $-20^{\circ} \mathrm{C}$.

\section{Large scale purification}

For larger quantities of plasmids, midi preps $(\sim 100 \mu \mathrm{g})$ using the low-copy plasmid purification protocol from the Nucleobond PC100 kit (Macherey-Nagel) was utilized. $100 \mathrm{~mL}$ of $E$. coli overnight culture were pelleted by centrifugation at $4000 \mathrm{rpm}$ for 15 min at $4{ }^{\circ} \mathrm{C}$ in a Sigma $3 \mathrm{~K} 18$ centrifuge. The pellet was resuspended in RNase-containing buffer, and cells were lyzed with 1\% SDS, $200 \mathrm{mM} \mathrm{NaOH}$ buffer, the alkaline conditions of which denatured both the chromosomal and plasmid DNA. The plasmid DNA returned to its soluble supercoiled state, while the genomic DNA and cellular debris precipitated upon neutralization of the reaction with buffer containing containing $2.8 \mathrm{M}$ potassium acetate $\mathrm{pH}$ 5.1. After clearing the lysate by centrifugation at $4000 \mathrm{rpm}$ for $40 \mathrm{~min}$ at $4^{\circ} \mathrm{C}$, it was loaded onto an equilibrated anion exchange column containing NucleoBond ${ }^{\circledR}$ AX Resin to which the DNA bound. The DNA was washed with buffer at $\mathrm{pH} 6.3$ which contained $100 \mathrm{mM}$ Tris, $1.15 \mathrm{M} \mathrm{KCl}$, and 15\% ethanol. The DNA was then eluted with $100 \mathrm{mM}$ Tris, $1 \mathrm{M} \mathrm{KCl}$, and $15 \%$ ethanol buffer adjusted to $\mathrm{pH}$ 8.5. The DNA was 
precipitated by addition of RT isopropanol followed by centrifugation for $40 \mathrm{~min}$ at 13000 rpm, $4{ }^{\circ} \mathrm{C}$ using the SS34 rotor of the Du Pont Sorvall RC-5B Refrigerated Superspeed Centrifuge. The pellet was resuspended in $500 \mu \mathrm{L}$ elution buffer to which $350 \mu \mathrm{L}$ isopropanol was added to precipitate the DNA again. This was centrifuged for $30 \mathrm{~min}$ at $15000 \mathrm{rpm}$ at $4{ }^{\circ} \mathrm{C}$ in a refrigerated Biofuge Fresco (Heraeus Instruments) tabletop centrifuge. The DNA pellet was washed with $500 \mu \mathrm{L} 70 \%$ ethanol and centrifuged at $13000 \mathrm{rpm}$ for $5 \mathrm{~min}$ at $\mathrm{RT}$ in a tabletop centrifuge. The ethanol was removed and the pellet was dried at RT for $20 \mathrm{~min}$. The pellet was then resuspended in $40 \mu \mathrm{l}$ sterilized water or TE buffer $\mathrm{pH} 8.5$ and stored at $-20{ }^{\circ} \mathrm{C}$.

\subsubsection{Concentration determination and sequencing of plasmid DNA}

\section{Measurement of DNA concentration}

DNA concentrations were determined spectroscopically using an ND-1000 spectrophotometer from NanoDrop Technologies Inc. (Rockland, USA) which has a DNA concentration range from $1.5 \mathrm{ng} / \mu \mathrm{L}$ to $3700 \mathrm{ng} / \mu \mathrm{L}$ and uses a path length of $0.2 \mathrm{~mm}$.

\section{Sequencing of the DNA constructs}

Plasmid DNA (see Table 2.4) containing the inserted gene in its multiple cloning site was sequenced by Eurofin MWG Operon (Ebersberg, Germany) using the sequencing primers (Table 2.5). The sequencing results were analyzed with a nucleotide BLAST (Basic sequence alignment search tool) (http://www.ncbi.nlm.nih.gov/BLAST). The nucleotide sequence of the construct was then translated into its protein sequence using the Translate Tool in SwissProt (http://www.expasy.org/tools/dna.html) and was then compared with the original cDNA sequence using CLUSTALW (http://www.ebi.ac.uk/clustalw/index.html).

Table 2.4: Plasmids used in this study

\begin{tabular}{|c|c|c|c|}
\hline Plasmid & Purpose & Antibiotic resistance & Source \\
\hline pETDuet-1 & $\begin{array}{c}\text { Expression of N-terminal His }(6) \text {-tag for } \\
\text { purification \& 2 multiple cloning sites }\end{array}$ & Ampicillin & Novagen \\
\hline pET-28a & $\begin{array}{c}\text { Expression of N-terminal His }(6) \text {-tag } \\
\text { with thrombin cleavage site }\end{array}$ & Kanamycin & Novagen \\
\hline pGEX-4T-1 & Expression of N-terminal GST-tag & Ampicillin & GE Healthcare \\
\hline pJET1/1.2 & Subcloning & Ampicillin & Fermentas \\
\hline
\end{tabular}

Table 2.5: Primers for sequencing plasmid inserts 


\begin{tabular}{|l|l|l|c|}
\hline Primer & Direction of sequencing & Plasmid & MCS \\
\hline pJET1.2 forward & forward 5' ---> 3' & pJET1.2 & 1 \\
\hline pJET1.2 reverse & reverse $3^{\prime}$---> 5' & pJET1.2 & 1 \\
\hline pET upstream & forward 5' ---> 3' & pETDuet-1 & 1 \\
\hline DuetDOWN1 & reverse 3' ---> 5' & pETDuet-1 & 1 \\
\hline DuetUP2 & forward 5' ---> 3' & pETDuet-1 & 2 \\
\hline T7 terminator & reverse 3' ---> 5' & pETDuet-1 & 2 \\
\hline pGEX forward & forward 5' ---> 3' & pGEX-4T-1 & 1 \\
\hline pGEX reverse & reverse 3' ---> 5' & pGEX-4T-1 & 1 \\
\hline pET upstream & forward 5' ---> 3' & pET-28a & 1 \\
\hline T7 terminator & reverse 3' ---> 5' & pET-28a & 1 \\
\hline
\end{tabular}

$\mathrm{MCS}=$ Multiple cloning site

\subsection{Protein Expression and Purification General Protocols}

Unless otherwise noted, the Äktaprime system from GE-Healthcare was used for automated protein purification for which all solutions were sterile-filtered ( $0.2 \mu \mathrm{m}$ filters) and degassed. The $\mathrm{OD}_{280}$ was measured and recorded with a chart recorder of the Äktaprime system or with the corresponding Unicorn 5.11 software (GE Healthcare). Columns were always washed thoroughly with double distilled water before equilibration in buffer. Before storage, columns were thoroughly washed with double distilled water before being stored in 20\% ethanol (analysis grade: 99.9\%).

\subsubsection{Overexpression and cell lysis}

\section{Recombinant protein over-expression}

For protein expression, the expression plasmids pET-28a, pETDuet-1, and pGEX-4T-1 were transformed into competent B121(DE3) or Rosetta 2(DE3) Escherichia coli cells. Rosetta 2(DE3) E. coli contain a chloramphenicol-selectable plasmid that encodes rare tRNAs for the expression of eukaryotic proteins. An overnight E. coli culture was used to inoculate autoinducible media (1:100 overnight culture:fresh media) (Studier 2005) containing the appropriate antibiotics for plasmid selection (see Table 2.4). ZYM-5052 autoinducible media contained both glucose and lactose. The glucose was preferentially metabolized by $E$. coli. Once the glucose was depleted, lactose was taken up by the cell and displaced the repressor on the lac operon of both the T7 RNA polymerase controlled by the lacUV5 promoter and encoded in the chromosomal DNA of $\lambda$ DE3 E.coli 
expression strains as well as that located just downstream of the $\mathrm{T} 7$ promoter region of the pET plasmids (Studier 1986 and 1990, Dubendorff 1991). The T7 RNA polymerase bound to the $\mathrm{T} 7$ promoter on the pET plasmid. With its high rate of transcription overexpression of the desired gene product resulted. In the case that Terrific Broth (TB: $52 \mathrm{~g}$ tryptone/peptone, $24 \mathrm{~g}$ yeast extract up to $1 \mathrm{~L}+$ salts at final concentration of $72 \mathrm{mM}$

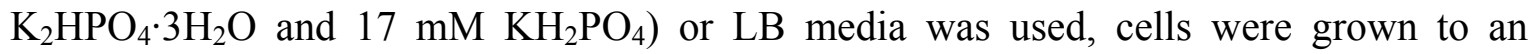
optical density at $600 \mathrm{~nm}\left(\mathrm{OD}_{600}\right)$ of $0.6-0.8$ before expression of the recombinant protein was induced by adding isopropyl- $\beta$-D-thiogalactoside (IPTG) (Fermentas) to a final concentration of $1 \mathrm{mM}$. IPTG is similar to lactose and therefore displaces the repressor on the lac operon. Transcription of the T7 RNA polymerase under control of the lac promoter is thus initiated.

The E. coli cultures were grown by shaking at 140-170 rpm (Infors Multitron shaker) at $37{ }^{\circ} \mathrm{C}$ until they reached an optical density between 0.5 and 0.7 at $600 \mathrm{~nm}$. Cultures were then transferred to $18{ }^{\circ} \mathrm{C}$ and harvested after $\sim 24$ hours by centrifuging at $4000 \mathrm{rpm}$ for $15 \mathrm{~min}$ at $4{ }^{\circ} \mathrm{C}$ using the JS-4.2 rotor in a Beckman J6-MI centrifuge. The supernatant was removed. The cells were resuspended in $\sim 12 \mathrm{~mL}$ of wash buffer (for corresponding affinity chromatography) per liter culture and stored at $-20{ }^{\circ} \mathrm{C}$ until needed.

\section{Bacterial cell lysis}

Resuspended cells were thawed and the following components were added: a protease inhibitor tablet (Roche) to prevent protein degradation, lysozyme (Roth) to stimulate lysis of the bacterial cell walls, and DNaseI (Applichem) to degrade DNA. Cells were lysed either with a French press (Constant Systems), a Bioruptor cell disruptor (Diagenode) with the output selector set to "high" by disrupting for $5 \mathrm{~min}$ at 30 second intervals 3 times total at $4{ }^{\circ} \mathrm{C}$, or with a Branson Sonifier 450 sonicator using a duty cycle of 50 and the output control set to the microtip limit which yielded an output of $20-30 \%$. After lysis, $1 \% \mathrm{v} / \mathrm{v}$ Triton-X 100 and $1 \mathrm{mM} \mathrm{MgCl}_{2} \cdot 6 \mathrm{H}_{2} \mathrm{O}$ (final concentration) were added. The lysed bacteria were incubated for 30 to $45 \mathrm{~min}$ at $4{ }^{\circ} \mathrm{C}$ to allow DNase activity and then centrifuged (Du Pont Sorvall) at $13000 \mathrm{rpm}$ using the SS34 rotor for $60 \mathrm{~min}$ at $4{ }^{\circ} \mathrm{C}$. The cleared lysate (supernatant) was loaded onto the column with either a $150 \mathrm{~mL}$ or $50 \mathrm{~mL}$ Superloop (GE-Healthcare) depending on the sample volume. 


\subsubsection{Affinity chromatography and cleavage of the affinity tag}

\section{Immobilized metal affinity chromatography (IMAC)}

Hexahistidine-tagged proteins were purified by immobilized metal affinity chromatography (Hochuli 1990, Sharma 1991). Nickel (Ni)-sepharose (GE-Healthcare) was used as column material and is comprised of highly cross-linked agarose coupled with a chelating group. $\mathrm{Ni}^{2+}$ binds to the chelating group. The column was equilibrated in a low imidazole, high salt washing buffer $\left(0.3 \mathrm{M} \mathrm{NaCl}, 50 \mathrm{mM} \mathrm{NaH}{ }_{2} \mathrm{PO}_{4} \cdot \mathrm{H}_{2} \mathrm{O}, 30-35 \mathrm{mM}\right.$ imidazole, $\mathrm{pH} 7.5$ ) to allow binding of the target protein to the $\mathrm{Ni}^{2+}$-sepharose. The cell supernatant was applied to the column at a flow rate of 0.5 to $1 \mathrm{~mL} / \mathrm{min}$. His-tagged proteins were retained on the column, whereas most nonspecific proteins in the lysate without a His-tag were washed through. After thorough washing with 10 to $25 \mathrm{CV}$ of washing buffer at 1 to $2 \mathrm{~mL} / \mathrm{min}$, a gradient of increasing concentration of imidazole ( 0 to $40 \%$ elution buffer) was applied at 0.5 to $1 \mathrm{~mL} / \mathrm{min}$ over 10 to $25 \mathrm{CV}$. The high imidazole elution buffer $\left(0.3 \mathrm{M} \mathrm{NaCl}, 50 \mathrm{mM} \mathrm{NaH} \mathrm{PO}_{4} \cdot \mathrm{H}_{2} \mathrm{O}, 1 \mathrm{M}\right.$ imidazole, $\mathrm{pH}$ 7.5) competed with the His-tagged proteins for binding to the $\mathrm{Ni}^{2+}$. The His-tagged proteins typically eluted at an imidazole concentration between 100 and $300 \mathrm{mM} .4 \mathrm{~mL}$ fractions were collected during elution. Afterwards the column was washed in $100 \%$ elution buffer to detach any remaining tightly bound protein $(1 \mathrm{~mL} / \mathrm{min}, 10-15 \mathrm{CV})$.

$1 \mathrm{~mL}$ His Trap ${ }^{\mathrm{TM}} \mathrm{FF}$ (GE Healthcare) columns were also utilized either alone or in series for small-scale purifications. The column was equilibrated with washing buffer (same as for Ni-sepharose). The supernatant was loaded onto the column using a $50 \mathrm{~mL}$ Superloop (GE-Healthcare) at a flow rate of $0.5 \mathrm{~mL} / \mathrm{min}$. The column was then washed with $20 \mathrm{CV}$ washing buffer at $1 \mathrm{~mL} / \mathrm{min}$ followed by an elution gradient of $0-100 \%$ over 30-50 CV elution buffer (0.3 M NaCl, $50 \mathrm{mM} \mathrm{NaH} \mathrm{PO}_{4} \cdot \mathrm{H}_{2} \mathrm{O}, 400 \mathrm{mM}$ imidazole, $\mathrm{pH}$ 7.5) at $1 \mathrm{~mL} / \mathrm{min}$ while collecting $1-2 \mathrm{~mL}$ fractions.

\section{Batch purification}

For the performance of simultaneous test purifications, a batch purificaiton with Ni-sepharose beads (GE Healthcare) was performed. The beads were first resuspended in their slurry then gently spun down at $2500 \mathrm{rpm}$ at $4{ }^{\circ} \mathrm{C}$ for 4 minutes and the ethanol was removed from the pelleted beads. The beads were then resuspended in an equal amount of Ni-washing buffer (see above). Beads were added to the supernatant 1:10 and incubated at $4{ }^{\circ} \mathrm{C}$ with gentle rolling for $90 \mathrm{~min}$. The beads were spun down at $3000 \mathrm{rpm}, 4{ }^{\circ} \mathrm{C}$ for 5 min and the supernatant containing all unbound proteins (flow-through) was removed. 
Washing buffer was added to the beads at an equal volume to the original amount of supernatant and incubated at $4{ }^{\circ} \mathrm{C}$ with gentle rolling for $10 \mathrm{~min}$. The washing step was repeated once more. Wash buffer was added to small glass column $(\sim 1 \mathrm{~cm}$ diameter $)$ and beads were added and allowed to settle. Wash buffer was allowed to flow through until just at the upper edge of the beads. Added 5\% volume elution buffer (see HisTrap ${ }^{\mathrm{TM}} \mathrm{FF}$ above) compared to the original amount of supernatant and stopped up column so that it could incubate for $30 \mathrm{~min}$ at $4^{\circ} \mathrm{C}$. The eluate was collected and this was repeated twice more with $10 \mathrm{~min}$ incubations each.

\section{Glutathione sepharose (GSH) purification}

A column packed with Glutathione Sepharose ${ }^{\mathrm{TM}} 4$ Fast Flow beads (GE Healthcare) was used with the Äktaprime system (GE Healthcare) for the purification of glutathione-S-transferase (GST)-tagged fusion proteins (Smith 1988). The self-packed glutathione-sepharose column was equilibrated with binding/wash buffer $(200 \mathrm{mM} \mathrm{NaCl}$, $30 \mathrm{mM}$ Hepes $\mathrm{pH} 7.5,2 \mathrm{mM}$ DTT). Cell lysate was applied to the column using a $50 \mathrm{~mL}$ superloop at a flow rate of $0.5 \mathrm{~mL} / \mathrm{min}$. Glutathione is a substrate for GST therefore proteins with a GST-tag bound to the glutathione-coupled beads. The column was then washed with $200 \mathrm{~mL}$ binding buffer to eliminate non-specific binding of other proteins. GST-proteins were eluted by adding $50 \mathrm{~mL}$ elution buffer containing free reduced glutathione $(200 \mathrm{mM} \mathrm{NaCl}, 30 \mathrm{mM}$ Hepes pH 7.5, $2 \mathrm{mM}$ DTT, $10 \mathrm{mM}$ reduced glutathione), which competitively bound to the GST-tag of the fusion protein, thus causing the fusion protein to dissociate from the GSH beads. The $\mathrm{pH}$ of the elution buffer was adjusted after addition of glutathione because the peptide is acidic. The eluate was collected in $2 \mathrm{~mL}$ fractions. Afterwards the column was washed with sterile-filtered water followed by $6 \mathrm{M}$ guanidine hydrochloride.

\section{His-tag cleavage by thrombin}

The His-tag introduced by the pET-28a plasmid can be cleaved using the thrombin cleavage site upstream between the tag and the sequence encoding the protein (Takagi 1974, reviewed in Jenny 2003). For the murine Atg5 and murine Rab33B(30-202) Q92L constructs in MCS1 of pETDuet-1, a thrombin cleavage site was introduced during PCR by the primer sequence. In this way, the His-tags could be removed from murine Atg5, murine Atg16L1, and murine Rab33B(30-202) Q92L after IMAC. Pooled fractions were dialyzed at $4{ }^{\circ} \mathrm{C}$ overnight in a Spectra/Pore dialysis membrane (Spectrumlabs.com) with gel filtration buffer containing $10 \mathrm{U}$ of thrombin (MP Biomedicals) per mg of protein. 
After dialysis, thrombin was inhibited by adding $4 \mathrm{mM}$ Pefabloc SC (Roche), a serine protease inhibitor.

\subsubsection{Anion exchange chromatography}

Ion exchange chromatography resolves charged particles and proteins based on charge differences. It does this by taking advantage of the ionization state of charged groups on the surface of a protein being highly dependent on the $\mathrm{pH}$ of the buffer that it is in. When in a buffer at a $\mathrm{pH}$ above its isoelectric point, a protein will have a negative net surface charge allowing it to bind to positively charged anion exchange beads. The Mono Q 5/50 GL column (GE Healthcare) has a column volume of $1 \mathrm{~mL}$. The column was equilibrated in 5-10 CV of low salt binding buffer ( $0.2 \mathrm{M} \mathrm{NaCl}, 20 \mathrm{mM}$ Tris $\mathrm{pH} \mathrm{8.0)}$. The elution fractions from a previous affinity chromatography purification were dialyzed overnight in binding buffer then loaded onto the column at $0.5 \mathrm{~mL} / \mathrm{min}$. The column was washed with $5-10 \mathrm{CV}$ of binding buffer at $0.7 \mathrm{~mL} / \mathrm{min}$ to wash through positively charged or only slightly negatively charged proteins that did not bind to the positively charged beads. The protein of interest was eluted by increasing the ionic strength of the buffer through a gradient from 0 to $100 \%$ elution buffer which contained high salt $(1 \mathrm{M} \mathrm{NaCl}, 20$ $\mathrm{mM}$ Tris $\mathrm{pH} 8.0$ ) over $20 \mathrm{CV}$. $0.5 \mathrm{~mL}$ fractions were collected during the gradient and the column was then cleaned with $2 \mathrm{CV} 100 \%$ elution buffer.

\subsubsection{Size exclusion chromatography}

Size exclusion chromatography was used as a final polishing step to remove aggregated proteins. This was done by resolving proteins based on molecular weight thus improving purity and by distinguishing which aggregated proteins and allowing their removal. For separation of proteins from 3-70 kDa, a HiLoad ${ }^{\mathrm{TM}}$ 16/60 Superdex $^{\mathrm{TM}} 75$ prep grade column from GE Healthcare was used. For resolution of proteins ranging from 10 to $600 \mathrm{kDa}$, a HiLoad ${ }^{\mathrm{TM}}$ 16/60 Superdex ${ }^{\mathrm{TM}} 200$ prep grade column was utilized in combination with the automated Äktaprime system (GE Healthcare). The column was equilibrated in gel filtration buffer (typically 0.15-0.3 M NaCl, 20-30 mM HEPES pH 77.5). The protein was concentrated prior to loading since only a maximum of $5 \mathrm{~mL}$ sample volume can be injected onto the HiLoad ${ }^{\mathrm{TM}}$ 16/600 Superdex ${ }^{\mathrm{TM}}$ column. This was done using Vivaspin 2 or 20 concentrators (Vivaspin Science Sartorius Group) with a molecular weight cut-off of either 3000,10000 , or 30000 depending on the size of the protein. Once concentrated, the sample was loaded via syringe into a $5 \mathrm{~mL}$ loading loop. 
The proteins were eluted from the column in a size-dependent manner with $130 \mathrm{~mL}$ gel filtration buffer at a flow rate of $0.5-1 \mathrm{~mL} / \mathrm{min} .2 \mathrm{~mL}$ fractions were collected beginning after $30 \mathrm{~mL}$. The void volume was at $\sim 40 \mathrm{~mL}$.

For the purification of less than $10 \mathrm{mg}$ of protein in up to $500 \mu \mathrm{L}$ sample volume, the smaller Superdex ${ }^{\mathrm{TM}} 200$ 10/300 GL column (GE Healthcare) was utilized with a maximum flow rate of $1 \mathrm{~mL} / \mathrm{min}$. The column was first washed with $2 \mathrm{CV}$ sterile-filtered water and then equilibrated with $2 \mathrm{CV}$ gel filtration buffer (see above). $1.25 \mathrm{CV}$ of gel filtration buffer were applied to the column at $0.5 \mathrm{~mL} / \mathrm{min}$ and $0.5 \mathrm{~mL}$ fractions were collected.

\subsubsection{Electrophoresis and concentration determination}

\section{Laemmli SDS-PAGE gel electrophoresis}

After each purification step, samples were analyzed by sodium dodecyl sulfate polyacrylamide gel electrophoresis (SDS-PAGE) (Laemmli 1970) which separates proteins according to their molecular weight. For an expression analysis, $200 \mu \mathrm{L}$ samples of the uninduced and harvested cultures were spun down at $13000 \mathrm{rpm}$ for 1 minute at RT. The supernatant was removed and the pellet was resuspended in $80 \mu \mathrm{L}$ water to which 40 $\mu \mathrm{L}$ of $3 \mathrm{x}$ loading buffer $(10 \mathrm{ml}$ contained $0.9 \mathrm{~g}$ SDS, $3 \mathrm{~g}$ glycerol, $1.8 \mathrm{~mL} 1 \mathrm{M}$ Tris $\mathrm{pH} 6.8$, $1 \mathrm{~mL} \beta$-mercaptoethanol) was added. The $\beta$-mercaptoethanol reduced disulfide-bridges.

During protein purification, $80 \mu \mathrm{L}$ samples of the cleared lysate (supernatant), pellet resuspended in water, and flow-through during supernatant injection onto the column were collected and combined with $40 \mu \mathrm{L}$ water and $60 \mu \mathrm{L} 3 \mathrm{x}$ loading buffer. Similarly, $20 \mu \mathrm{L}$ samples of the elution fractions were combined with $10 \mu \mathrm{L} 3 \mathrm{x}$ loading buffer. Samples were then heated for $3-5 \mathrm{~min}$ at $95^{\circ} \mathrm{C}$.

The SDS-PAGE gel consists of two parts: a $3.75 \%$ stacking gel and $5-15 \%$ separating gels. $12 \%$ and $15 \%$ separating gels were used in this study (Table 2.6). For the combs to make 15 wells/gel, $10 \mu \mathrm{L}$ protein samples were loaded into each well of the stacking gel. The gels were run with at $150-200 \mathrm{~V}$ using the Mini-Protean 3 Electrophoresis Cell system from Biorad using Laemmli running buffer (250 mM Tris, 1.9 M glycine, $1 \%$ SDS). The SDS coating on the proteins gave it a charge:mass ratio causing the protein to travel down the gel toward the positive electrode when an electric field is applied. The proteins moved quickly through the stacking gel; however, they were slowed when they reached the edge to the separating gel. Due to the $\mathrm{pH}$ difference between the 
stacking and separating gels, the sample became concentrated at the border, which sharpened the bands. Heavier proteins move more slowly through the pores than smaller proteins. Increasing the concentration of polyacrylamide in the separating gel resulted in better resolution of large proteins.

Table 2.6: Laemmli SDS-PAGE gel components

\begin{tabular}{|c|c|c|c|c|}
\hline Stacking gel & $3.75 \%$ & Separating gel & $12 \%$ & $15 \%$ \\
\hline Upper gel buffer (pH 6.8) & $2.5 \mathrm{~mL}$ & Lower gel buffer (pH 8.8) & $2.5 \mathrm{~mL}$ & $2.5 \mathrm{~mL}$ \\
\hline $\mathrm{dH}_{2} \mathrm{O}$ & $6.14 \mathrm{~mL}$ & $\mathrm{dH} 20$ & $3.4 \mathrm{~mL}$ & $2.4 \mathrm{~mL}$ \\
\hline $30 \%$ AMBA & $1.25 \mathrm{~mL}$ & $30 \%$ AMBA & $4.0 \mathrm{~mL}$ & $5.0 \mathrm{~mL}$ \\
\hline TEMED & $10 \mu \mathrm{L}$ & TEMED & $5 \mu \mathrm{L}$ & $5 \mu \mathrm{L}$ \\
\hline $10 \%$ APS & $100 \mu \mathrm{L}$ & $10 \%$ APS & $100 \mu \mathrm{L}$ & $100 \mu \mathrm{L}$ \\
\hline \multicolumn{5}{|c|}{$\begin{array}{l}\text { *makes } 4 \text { gels } \\
\text { Upper gel buffer }(0.5 \mathrm{M} \text { Tris, } 0.4 \% \mathrm{SDS}) \\
\text { Lower gel buffer }(1.5 \mathrm{M} \text { Tris, } 0.4 \% \mathrm{SDS} \\
\text { AMBA=acrylamide/N,N'-methylene-bis-acrylamide }(30 \% \text { acrylamide, } 0.8 \% \text { bisacrylamide stock }) \\
\text { APS }=\text { ammonium persulfate }(10 \% \text { stock }) \\
\text { TEMED=tetramethylethylenediamine }\end{array}$} \\
\hline
\end{tabular}

\section{Schägger Jagow SDS-PAGE gel electrophoresis}

Schägger Jagow gel electrophoresis (Schägger 1987) can separate proteins in a total range from 1 to $100 \mathrm{kDa}$ with the optimal resolution for proteins smaller than $30 \mathrm{kDa}$. The use of tricine in the cathode buffer has a lower negative charge than glycine used in the Laemmli SDS-PAGE running buffer. Its higher ionic strength causes it to travel faster through the gel. This results in a higher resolution of proteins $\leq 10 \mathrm{kDa}$ because the ions move more quickly through the lower part of the gel than the low molecular weight proteins, even at lower polyacrylamide concentrations. This system consists of both a separating gel on the bottom and a stacking gel on the top (see Table 2.7). Protein samples to be loaded onto gels were combined with 2x Schägger Jagow sample buffer (50 mM Tris to $\mathrm{pH} 6.8$ with $\mathrm{HCl}, 4 \% \mathrm{SDS}, 0.01 \%$ Serva Blue G, $12 \%$ glycerol, $2 \% \beta$-mercaptoethanol) and boiled for $3-5 \mathrm{~min}$ at $95^{\circ} \mathrm{C}$. Cathode buffer $(0.1 \mathrm{M}$ Tris, $0.1 \mathrm{M}$ Tricine, $1 \% \mathrm{SDS})$ was placed on the inner portion of the apparatus between the glass plates and anode buffer $(0.2$ $\mathrm{M}$ Tris, $\mathrm{pH}$ 8.9) was placed on the outside of the glass plates. The Mini-Protean 3 Electrophoresis Cell system from Biorad was utilized. The gels were run with at $70 \mathrm{~V}$ until the proteins were in the separating gel then they were run at $120 \mathrm{~V}$ for the remainder. 
Table 2.7. Schägger Jagow gel components

\begin{tabular}{|c|c|c|}
\hline & 4\% Stacking gel & $10 \%$ Separating gel \\
\hline 30\% АMBA & $400 \mu \mathrm{L}$ & $3.32 \mathrm{~mL}$ \\
\hline Gel buffer & $750 \mu \mathrm{L}$ & $3.35 \mathrm{~mL}$ \\
\hline $\mathrm{ddH}_{2} \mathrm{O}$ & $1.85 \mathrm{~mL}$ & $1.14 \mathrm{~mL}$ \\
\hline $50 \%$ glycerol & ------------- & $2.12 \mathrm{~mL}$ \\
\hline TEMED & $4 \mu \mathrm{L}$ & $6 \mu \mathrm{L}$ \\
\hline $10 \%$ APS & $20 \mu \mathrm{L}$ & $50 \mu \mathrm{L}$ \\
\hline \multicolumn{3}{|c|}{$\begin{array}{l}\text { *makes } 2 \text { gels } \\
\text { Gel buffer=3M Tris adjusted to } \mathrm{pH} 8.45 \text { with } \mathrm{HCl}, 0.3 \% \mathrm{SDS} \\
\text { AMBA=Acrylamide/N,N'-methylene-bis-Acrylamide } \\
\text { AMBA=30\% acrylamide stock solution with } 0.8 \% \text { bisacrylamide } \\
\qquad \mathrm{APS}=\text { Ammonium persulfate } \\
\qquad \text { TEMED=Tetramethylethylenediamine }\end{array}$} \\
\hline
\end{tabular}

\section{Molecular markers and staining/destaining solutions}

A $15 \%$ SDS PAGE is suitable to analyze proteins with molecular weights between 10 to $100 \mathrm{kDa}$. Molecular weights of the bands were estimated using various molecular markers from Fermentas (catalog no. SM0431, SM0671, SM1861, SM1881) and Sigma (M3913). Gels were stained with Coomassie Brilliant Blue solution $(0.25 \% \mathrm{w} / \mathrm{v}$ Coomassie Blue, $7.5 \%$ acetic acid, $40 \%$ ethanol) by boiling it for one minute in a microwave. After cooling to RT, the gel was moved into a destaining solution $(10 \%$ acetic acid, 40\% ethanol) and left shaking overnight. A faster variation of this adopted from the Prof. Brose lab (Goettingen, Germany) involves staining solutions A (500 mg CoomassieR, $650 \mathrm{~mL} \mathrm{H}$ H, $250 \mathrm{~mL}$ isopropanol, $100 \mathrm{~mL}$ acetic acid), B (50 mg CoomassieR, $800 \mathrm{~mL} \mathrm{H}$ H, $100 \mathrm{~mL}$ isopropanol, $100 \mathrm{~mL}$ acetic acid), C (20 mg CoomassieR, $900 \mathrm{~mL} \mathrm{H}_{2} \mathrm{O}, 100 \mathrm{~mL}$ acetic acid), and destaining solution $\mathrm{D}\left(900 \mathrm{~mL} \mathrm{H}_{2} \mathrm{O}\right.$, $100 \mathrm{~mL}$ acetic acid). The gel was covered with staining solution $\mathrm{A}$ and heated for $1 \mathrm{~min}$ in a microwave. Staining solution away was removed and staining solution B was used to cover the gel which was then heated in the microwave for $1 \mathrm{~min}$. This procedure was repeated for staining solution $\mathrm{C}$ followed by destaining solution D. The gel was then left shaking gently at RT for 15-30 min before the destaining solution was removed and replaced with water. Visualization within $40 \mathrm{~min}$ was sometimes achieved using PageBlue Protein Staining Solution (\#R0571) from Fermentas.

\section{Measurement of protein concentration}

Protein concentrations were usually determined spectroscopically using an ND1000 spectrophotometer from NanoDrop Technologies Inc., Rockland, USA which used a path length of $0.2 \mathrm{~mm}$ and measured the absorbance of aromatic amino acid residues at a 
wavelength of $280 \mathrm{~nm}$ using the molecular weight and extinction coefficient of the protein.

For proteins like Rab33BQ92L which had GTP bound which absorbs at $260 \mathrm{~nm}$, protein concentrations were determined using the Bradford assay (Bradford 1976) to avoid interference of nucleotide absorbance. A standard curve was calculated based on 1, 2, 3, $4,5,7,8,9$, and $10 \mu \mathrm{g}$ BSA. This protein was added to sterile water with a final volume

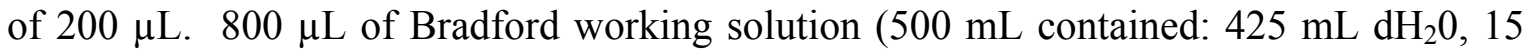
$\mathrm{mL} 95 \%$ ethanol, $30 \mathrm{~mL} \mathrm{88 \%}$ phosphoric acid, $30 \mathrm{~mL}$ Bradford stock $\{100 \mathrm{~mL} 95 \%$ ethanol, $200 \mathrm{~mL} \mathrm{88 \%} \mathrm{phosphoric} \mathrm{acid,} 350 \mathrm{mg}$ Serva Blue G) which is in its protonated form and has a reddish-brown in color was added to the $200 \mu \mathrm{L}$ standard protein. Protein binding stabilizes the dye in its anionic state resulting in a color change to blue, and the intensity depends on the concentration of protein. The absorbance of the mixture was measured at $595 \mathrm{~nm}$ on a Genesys 6 spectrophotomer (Thermo Spectronic). The concentration of the protein was determined from the BSA standard curve.

\subsubsection{Western blotting}

Immunoblotting was performed to detect a specific protein or fusion tag. First SDS-PAGE gel electrophoresis was performed to resolve the proteins based on molecular weight. The proteins were transferred onto a nitrocellulose membrane (Whatman, Schleicher and Schuell) using the semi-dry apparatus (Phase, Lübeck) (Towbin 1979, 1989). The SDS-PAGE gel and nitrocellulose membrane were washed with water and then transfer buffer (25 mM Tris, $192 \mathrm{mM}$ glycine, 0.04\% w/v SDS, 20\% v/v methanol) for 5-10 min each with gentle shaking. The filter paper was also equilibrated in transfer buffer.

A sandwich consisting from bottom to top of two sheets of filter paper, the SDSPAGE gel, the nitrocellulose membrane and then two additional filter papers on top was assembled. The cover of the semi-dry blot apparatus was attached and a current of $45 \mathrm{~mA}$ was applied. After blotting for 30-50 min at RT, the device was disassembled. The SDSPAGE gel was stained with Coomassie Brilliant Blue staining solution to check for efficient protein transfer. The membrane was rinsed in water for $5 \mathrm{~min}$ to remove SDS and transferred into TBS-Tween buffer $(150 \mathrm{mM} \mathrm{NaCl}, 10 \mathrm{mM}$ Tris $\mathrm{pH} 7.4,0.1 \%$ Tween20) for $5 \mathrm{~min}$ with gentle shaking. The nitrocellulose membrane was then placed in blocking buffer (TBS-Tween buffer supplemented with 5\% (w/v) instant skim milk powder) and blocked for 30-60 min at RT while shaking. 
Next the membrane was incubated with primary antibody diluted in blocking buffer at $4{ }^{\circ} \mathrm{C}$ overnight. After incubation with the first antibody the membrane was rinsed with TBS-Tween buffer 4 times for 5 min each and then blocked in blocking buffer for $20 \mathrm{~min}$. It was then transferred into fresh blocking buffer containing the secondary antibody, conjugated either to a fluorophore or horseradish peroxidase, and incubated for 45 min to 3 hours with gentle shaking. After that the membrane was washed 4 times with TBS-Tween buffer to remove unbound antibody.

If the secondary antibody was conjugated to a fluorophore, the fluorescence was detected using the Fujifilm FLA-7000 fluorescence detector using the FLA-7000 IR v. 1.1 software (Fujifilm $\subset$ 2006). The membrane was dried at RT and placed face down onto the glass. Fluorescence detection was chosen with $25 \mu \mathrm{m}$ pixel size and PMT 600 mode free.

If the secondary antibody was conjugated to HRP, the membrane was placed onto a glass plate and covered with Western Lightning ${ }^{\mathrm{TM}}$ Plus-ECL Oxidizing Reagent Plus 1:1 with Western Lightning ${ }^{\mathrm{TM}}$ Plus-ECL Luminol Reagent Plus (Perkin Elmer LAS) and shaken gently for $1 \mathrm{~min}$. The membrane was exposed and the image was recorded using the Imageready LAS-1000 CCD camera (Fujifilm) and corresponding software. Aida software (Fujifilm) was used to visualize the images and export them into other file formats.

\subsubsection{Protein stability assays}

\section{ThermoFluor Assay}

Thermal shift (ThermoFluor) assays were used to screen different buffers and additives for conditions and compounds that would increase the thermal stability of the protein complexes as evidenced by an increase in the melting temperature, $\mathrm{T}_{\mathrm{m}}$ (Pantoliano 2001, Lo 2004). $T_{m}$ was defined as the temperature midpoint between the transition from folded to unfolded state, the latter state being irreversible. This was measured using a real-time PCR machine (MJ Research) which detected the fluorescence of Sypro Orange ${ }^{\circledR}$ dye (Sigma, S5692). Sypro Orange fluorescence is quenched when in solution. However, as the protein was denatured upon gradual heating, Sypro Orange bound to the unfolded, interior hydrophobic regions of the proteins and its fluorescence was dequenched. Fluorescence is measured with real-time PCR machine as a function of temperature. 
The protein was first diluted to 20x the final desired concentration, which was usually between $0.75-1 \mathrm{mg} / \mathrm{mL}$, depending on the protein. The concentration was optimized for each protein in order to find the lowest concentration that produced a reliable signal. The Sypro Orange dye was then diluted from 5000x to 100x using the same buffer as for the protein. $18 \mu \mathrm{L}$ of buffer was added to empty wells of the 96 well plate (Biozym Multiplate 96 unskirted Low Profile \#621835) as a control. An equal volume of 100x Sypro Orange dye was added to the $20 \mathrm{x}$ protein and $2 \mu \mathrm{L}$ of this mixture was added to the wells of the 96-well plate. The plate was sealed with an adhesive aluminum foil and centrifuged for $5 \mathrm{~min}$ at $2000 \mathrm{rpm}, 4^{\circ} \mathrm{C}$. The foil was then peeled off and replaced by clear 8-er flat cap strips (Biozym). Opticon software was used to run a program from 25 to $91{ }^{\circ} \mathrm{C}$ with steps of $1{ }^{\circ} \mathrm{C}$ and 30 seconds break in between to read out the fluorescence intensitites.

The $\mathrm{pH}$ screen was developed by Erika Mancini and Christian Siebold (see Nettleship 2008) with the following alterations. The well that should have contained $\beta-\gamma-$ methylGTP was filled with buffer instead to use as a control. $2 \mathrm{mM}$ AMPccP was replaced with $2 \mathrm{mM} \alpha, \beta$-methyleneadenosine 5'- diphosphate sodium salt. AMPcPP was replaced with $2 \mathrm{mM} \alpha, \beta$-methyleneadenosine 5'-triphosphate lithium salt. $2 \mathrm{mM} \mathrm{AMPPcP}$ was replaced with $2 \mathrm{mM} \beta, \gamma$-methyleneadenosine 5'-triphosphate sodium salt. Buffer solutions were from Hampton Research and the compounds were from Sigma.

\section{Stability test}

After gel filtration, the mammalian His-Atg5 Atg16(1-231) complex was combined with gel filtration buffer $(0.2 \mathrm{M} \mathrm{NaCl}, 30 \mathrm{mM}$ HEPES $\mathrm{pH} 7.5)$ to yield a final concentration of $1 \mathrm{mg} / \mathrm{mL}$ (total volume $=500 \mu \mathrm{L}$ ). The protein was incubated at RT over 9 days. At each time point as indicated, $40 \mu \mathrm{L}$ of the protein was combined with $20 \mu \mathrm{L} 3 \mathrm{x}$ SDS-PAGE sample buffer and boiled at $95{ }^{\circ} \mathrm{C}$ for $2 \mathrm{~min}$.

\subsection{Protein-specific purification protocols}

\subsubsection{Mammalian His-Atg5}

Full-length human His-Atg5-pETDuet-1 in Rosetta 2 (DE3) E. coli was expressed in 1.5 L ZYM-5052 autoinducible media. After they reached an $\mathrm{OD}_{600}=0.5$ at $37^{\circ} \mathrm{C}$, the cells were put on ice for $30 \mathrm{~min}$ before being incubated at $18{ }^{\circ} \mathrm{C}$ for 24 hours. After harvest, the pellets were resuspended in binding/wash buffer: $0.3 \mathrm{M} \mathrm{NaCl}, 50 \mathrm{mM}$ 
$\mathrm{NaH}_{2} \mathrm{PO}_{4}, 30 \mathrm{mM}$ imidazole, $\mathrm{pH}$ 7.5. The general protocol for IMAC was followed. Fractions containing the protein were pooled and dialyzed against $2 \mathrm{~L}$ of Mono $\mathrm{Q}$ binding buffer overnight. The general protocol for anion exchange chromatography was then followed.

\subsubsection{Mammalian His-Atg5 His-Atg16L1 truncation complexes}

Murine His-Atg16L1-pET-28a truncation constructs were co-transformed with either full-length human or murine His-Atg5-pETDUET-1 (murine constructs included a thrombin cleavage site after the His-tag added by PCR) into Rosetta 2 (DE3) E. coli. An overnight culture of this was used to inoculate 3-6 L ZYM-5052 autoinducible media which grew to $\mathrm{OD}_{600}=0.5-0.6$ at $37{ }^{\circ} \mathrm{C}$ and then incubated at $18{ }^{\circ} \mathrm{C}$ for 24 hours. The general overexpression and lysis protocols were used. The general IMAC protocol was followed using either the column with Ni-sepharose beads or the HisTrap ${ }^{\mathrm{TM}}$ FF columns, usually two $1 \mathrm{~mL}$ columns connected in series. For His-Atg5 His-Atg16L1(1-265) IMAC washing buffer with an imidazole concentration of 35-40 $\mathrm{mM}$ was used since there were usually more non-specific proteins bound. After IMAC, pure fractions were pooled and dialyzed overnight in gel filtration buffer (with variation according to construct, see below) sometimes while having the His-tag cleaved by thrombin depending on whether the tag was needed for detection.

The complex was then concentrated and loaded onto a HiLoad ${ }^{\mathrm{TM}}$ 16/60 Superdex ${ }^{\text {TM }} 200$ prep grade column. For protein preps after August 2010, 1-5 mM $\mathrm{MgCl}_{2} \cdot 6 \mathrm{H}_{2} \mathrm{O}$ was included in all purification buffers since this seemed to have a stabilizing effect. Gel filtration buffers used were as follows: His-Atg5 His-Atg16L1(1$70)=0.2 \mathrm{M} \mathrm{NaCl}, 30 \mathrm{mM}$ HEPES pH 7.5, His-Atg5 His-Atg16L1(1-106, 1-113, 24$168,53-168,1-168,1-231)=0.15-0.2 \mathrm{M} \mathrm{NaCl}, 30 \mathrm{mM}$ HEPES pH 7.5, and His-Atg5 HisAtg16L1(1-265) $=0.2 \mathrm{M} \mathrm{NaCl}, 30 \mathrm{mM}$ HEPES pH 7.5. The ThermoFluor assay with the $\mathrm{pH}$ screen was used to optimize the buffer $\mathrm{pH}$ to increase stability of the Atg5 Atg16L1(1-70) and Atg5 Atg16L1(1-265) complexes. After this assay was performed, the $\mathrm{pH}$ of the buffer component was accordingly adapted. For the Atg5 Atg16L1(1-70) and Atg5 Atg16L1(1-265) complexes the buffer component of the gel filtration buffer was changed to $30 \mathrm{mM}$ MES pH 6.3 and $30 \mathrm{mM}$ HEPES pH 7.0, respectively. 


\subsubsection{Mammalian His-Atg5 His-Atg16L1(1-623)}

Rosetta 2 (DE3) E. coli were co-transformed with full-length human His-Atg5pETDuet-1 and His-Atg16L1(1-623)-pET-28a. Overnight culture was used to inoculate 4.5 L LB at a ratio of $1: 100(\mathrm{v} / \mathrm{v})$. After they reached $\mathrm{OD}_{600}=0.6$ at $37^{\circ} \mathrm{C}$, expression was induced with $1 \mathrm{mM}$ IPTG. The cells were incubated for an additional 3 hours at $37{ }^{\circ} \mathrm{C}$ before being harvested. After harvest, the pellets were resuspended in $6 \mathrm{~mL} \mathrm{Ni}$ binding/wash buffer per L grown culture. The general purification protocol for IMAC was followed.

\subsubsection{Mammalian GST-Atg16L1(328-623)}

GST-Atg16L1(328-623)-pGEX-4T-1-containing BL21 (DE3) E. coli were grown in $6 \mathrm{~L} \mathrm{~TB}$ containing $1: 100$ salts at $37^{\circ} \mathrm{C}$. After they reached $\mathrm{OD}_{600}=0.8$, expression was induced with $1 \mathrm{mM}$ IPTG. The cell shook for an additional 3.5 hours at $37{ }^{\circ} \mathrm{C}$ before being harvested. After harvest, the pellets were resuspended in GSH binding/wash buffer. The general protocol for GSH purification was followed. Fractions containing the protein were pooled. The sample was then concentrated $5 \mathrm{~mL}$ using a Vivaspin 20 with a molecular weight cutoff (MWCO) of 30000. The protein was then loaded onto a HiLoad $^{\mathrm{TM}}$ 16/60 Superdex ${ }^{\mathrm{TM}} 75$ gel filtration column for size exclusion chromatography using the following buffer: $0.2 \mathrm{M} \mathrm{NaCl}, 30 \mathrm{mM}$ HEPES pH 7.5, $1 \mathrm{mM}$ dithiotreitol (DTT) (Formedium ${ }^{\mathrm{TM}}$ ).

\subsubsection{Mammalian GST-Rab33B(1-229)Q92L}

GST-Rab33B(1-229) Q92L was transformed into BL21 (DE3) E. coli and inoculated from an overnight culture into $4.5 \mathrm{~L}$ TB media. The standard protocol for induction was followed with the cells being harvested after incubating 5 hours at $37{ }^{\circ} \mathrm{C}$ after being induced. Cells were lyzed by sonication twice for 2 min each with a $1 \mathrm{~min}$ pause in between. Magnesium chloride was added to a final concentration of $10 \mathrm{mM}$. $2.25 \mathrm{~mL}$ of equilibrated Glutathione Sepharose ${ }^{\mathrm{TM}} 4$ Fast Flow beads (GE Healthcare) in their slurry were added to $13 \mathrm{~mL}$ of supernatant and shaken gently for 2 hours at $4^{\circ} \mathrm{C}$. The beads were washed twice with $10 \mathrm{~mL}$ washing buffer $(200 \mathrm{mM} \mathrm{NaCl}, 30 \mathrm{mM}$ Hepes $\mathrm{pH}$ 7.5, $2 \mathrm{mM}$ DTT) and were spun down at $3000 \mathrm{rpm}$ for $5 \mathrm{~min}$ at $4{ }^{\circ} \mathrm{C}$ in between to remove the unbound protein. Beads were transferred into a small glass column ( $\sim 1 \mathrm{~cm}$ diameter $)$ containing wash buffer and allowed to completely settle. $10 \mathrm{~mL}$ elution buffer (glutathione $(200 \mathrm{mM} \mathrm{NaCl}, 30 \mathrm{mM}$ Hepes $\mathrm{pH}$ 7.5, $2 \mathrm{mM}$ DTT, $10 \mathrm{mM}$ reduced 
glutathione) was added and the column was stopped up and incubated for 10 min at $4{ }^{\circ} \mathrm{C}$. The eluate was collected and this was repeated twice more to elute all protein.

\subsubsection{Mammalian His-Rab33B(30-202) Q92L}

His-Rab33B(30-202) Q92L was transformed into Rosetta 2 (DE3) E. coli and inoculated from an overnight culture into 4.5 L ZYM-5052 and grown at $18{ }^{\circ} \mathrm{C}$ for 24 hours according to the general overexpression protocol. Cells were resuspended in wash buffer containing $500 \mathrm{mM} \mathrm{NaCl}, 50 \mathrm{mM}$ HEPES pH 7.5, 1-5 $\mathrm{mM} \mathrm{MgCl}_{2}, 2 \mathrm{mM} \beta-$ mercaptoethanol, and $10 \mu \mathrm{M}$ GTP with a final $\mathrm{pH}$ of 7.5. The general lysis protocol was followed with cells being lyzed by sonication 4 times 30 seconds each with 1 min pauses in between. For purification with the HisTrap ${ }^{\mathrm{TM}}$ FF columns, two columns were connected in series. During the washing steps, $5 \%$ of the elution buffer $(500 \mathrm{mM} \mathrm{NaCl}$, $50 \mathrm{mM}$ HEPES pH 7.5, $1 \mathrm{mM} \mathrm{MgCl} 2,2 \mathrm{mM} \beta$-mercaptoethanol, $10 \mu \mathrm{M}$ GTP, $500 \mathrm{mM}$ imidazole, $\mathrm{pH}$ 7.5) was added to reduce nonspecific binding. The protein was eluted with $40 \mathrm{~mL}$ elution buffer from $0-100 \%$ with $2 \mathrm{~mL}$ fraction being collected.

When the Ni-sepharose column was used instead, the elution buffer contained $1 \mathrm{M}$ imidazole and the elution gradient was set from $0-40 \%$ with $4 \mathrm{~mL}$ fractions being collected. Pure fractions were pooled and dialyzed overnight in gel filtration buffer $(0.3$ $\mathrm{M} \mathrm{NaCl}, 30 \mathrm{mM}$ HEPES pH 7.5, $1 \mathrm{mM} \mathrm{MgCl}_{2}, 2 \mathrm{mM} \beta$-mercaptoethanol, $10 \mu \mathrm{M}$ GTP). Size exclusion chromatography was performed using a HiLoad ${ }^{\mathrm{TM}}$ 16/60 Superdex $^{\mathrm{TM}} 75$ prep grade column. $2 \mathrm{~mL}$ fractions were collected during the isocratic elution over 1.25 CV.

\subsubsection{Mammalian His-Rab33B(30-202)Q92L Atg16L1 complexes}

$200 \mathrm{~mL}$ of ZYM-5052 was inoculated with bicistronic His-Rab33B(30-202) Q92L Atg16L1(141-265)-pETDuet1, His-Rab33B(30-202) Q92L Atg16L1(172-265)pETDuet1, or His-Rab33B(30-202) Q92L Atg16L1(172-234)-pETDuet1 transformed into Rosetta 2 (DE3) and grown out, harvested, resuspended in wash buffer (detailed below) and lyzed according to the general protocols. The His-Rab33B(30-202)Q92L complexes were first purified using the Ni batch method. The supernatant was added to a $15 \mathrm{~mL}$ Falcon tube to which $350-400 \mu \mathrm{L}$ equilibrated Ni beads were added. They shook gently for $1 \mathrm{hr}$ at $4{ }^{\circ} \mathrm{C}$. The beads were then gently spun down for $5 \mathrm{~min}$ at $3000 \mathrm{rpm}$ at $4{ }^{\circ} \mathrm{C}$. The flow-through with unbound proteins was removed. The beads washed with $5 \mathrm{~mL}$ wash buffer $(0.3 \mathrm{M} \mathrm{NaCl}, 50 \mathrm{mM}$ HEPES $\mathrm{pH} 7.5,30 \mathrm{mM}$ imidazole, $5 \mathrm{mM} \mathrm{MgCl} 2,10 \mu \mathrm{M}$ 
GTP, $2 \mathrm{mM} \beta$-mercaptoethanol, $\mathrm{pH}$ 7.3) for $5 \mathrm{~min}$ at $4{ }^{\circ} \mathrm{C}$ and spun down again. The supernatant was removed and this was repeated twice more. The bound protein was eluted by adding $500 \mu \mathrm{L}$ elution buffer ( $0.3 \mathrm{M} \mathrm{NaCl}, 50 \mathrm{mM}$ HEPES pH 7.5, $400 \mathrm{mM}$ imidazole, $5 \mathrm{mM} \mathrm{MgCl}_{2}, 10 \mu \mathrm{M}$ GTP, $2 \mathrm{mM} \beta$-mercaptoethanol, $\mathrm{pH}$ 7.3) and letting the beads shake gently for $10 \mathrm{~min}$ at $4{ }^{\circ} \mathrm{C}$ before being spun down at $4000 \mathrm{rpm}$ for $5 \mathrm{~min}$ at $4{ }^{\circ} \mathrm{C}$. The supernatant was collected as the first elution fraction. This was repeated twice more with $1 \mathrm{~mL}$ of elution buffer being used for the third elution fraction.

For larger scale preps, 4.5 L of ZYM-5052 were inoculated with the above bicistronic constructs and grown, harvested, and lyzed according to the general protocols. IMAC was employed for first step purification with two $1 \mathrm{~mL}$ HisTrap $^{\text {TM }}$ FF columns $(\mathrm{CV}=2 \mathrm{~mL})$ connected in series. The washing buffer is the same as above. The elution buffer is the same as above with the following alteration: $500 \mathrm{mM}$ imidazole was used for the elution gradient instead of isocratic elution with $400 \mathrm{mM}$ in the batch method. After the cleared cell lysate was loaded onto the column, the column was washed with $20 \mathrm{CV}$ washing buffer and eluted with a gradient from $0-100 \%$ over $20-25 \mathrm{CV}$. The eluate was collected in $2 \mathrm{~mL}$ fractions. The column was then washed with $100 \%$ elution volume over $5 \mathrm{CV}$ before being re-equilibrated in washing buffer for purification of the next complex.

Samples of the elution fractions were run on $15 \%$ SDS-PAGE gels. Those that contained the complex were pooled and dialyzed overnight at $4{ }^{\circ} \mathrm{C}$ in $0.2 \mathrm{M} \mathrm{NaCl}, 30 \mathrm{mM}$ HEPES pH 7.2, $5 \mathrm{mM} \mathrm{MgCl}_{2}, 10 \mu \mathrm{M}$ GTP, and $2 \mathrm{mM} \beta$-mercaptoethanol. The next morning, the dialyzed protein was concentrated down to $5 \mathrm{~mL}$ and loaded onto a HiLoad $^{\text {TM }}$ 16/60 Superdex ${ }^{\mathrm{TM}} 75$ prep grade column for size exclusion chromatography according to the general protocol. The gel filtration buffer used was as follows: $0.15 \mathrm{M}$ $\mathrm{NaCl}, 30 \mathrm{mM}$ HEPES pH 7.2, $5 \mathrm{mM} \mathrm{MgCl}_{2}, 10 \mu \mathrm{M}$ GTP, and $2 \mathrm{mM} \beta$-mercaptoethanol. The fractions were analyzed by SDS-PAGE gel and the non-aggregated Rab33B Q92L Atg16L1 complex-containing fractions were pooled. $2 \mathrm{mM}$ GTP was added to the pooled protein to assure that the concentration of GTP was sufficient and Rab33B would remain in its GTP bound form.

\subsubsection{Mammalian His-Atg5 His-Atg16L1(1-265) mutants}

Murine His-Atg16L1-pET-28a mutant constructs were co-transformed with either full-length murine His-Atg5-pETDUET-1 (a thrombin cleavage site after the His-tag was added by PCR) into Rosetta 2 (DE3) E. coli. An overnight culture of this was used to inoculate 1-1.2 L ZYM-5052 autoinducible media (4.5 L for the K14AR15AR22A triple 
mutant) which was grown to $\mathrm{OD}_{600}=0.5-.6$ and then incubated at $18{ }^{\circ} \mathrm{C}$ for 24 hours. The general overexpression and lysis protocols were followed. The general IMAC protocol was followed using $1 \mathrm{~mL}$ HisTrap $^{\mathrm{TM}} \mathrm{FF}$ columns, usually 2 in series. HisAtg5 Atg16L1(1-265) mutants had IMAC washing buffer containing $40 \mathrm{mM}$ imidazole. IMAC elution buffer contained $1 \mathrm{M}$ imidazole. The protein complex appeared to stick to the column therefore a gradient of up to $60 \%$ elution buffer was applied. After IMAC, pure fractions were pooled and dialyzed overnight in gel filtration buffer $(0.2 \mathrm{M} \mathrm{NaCl}, 30$ $\mathrm{mM}$ HEPES $\mathrm{pH}$ 7.0). The complex was then concentrated and underwent size exclusion chromatography on a HiLoad ${ }^{\text {TM }}$ 16/60 Superdex ${ }^{\text {TM }} 200$ prep grade column.

\subsubsection{Yeast His-Atg5 Atg16(1-46) \& Atg5 Atg16(1-57) complexes}

Saccharomyces cerevisiae His-Atg5/Atg16(1-46)pETDUET-1 or HisAtg5/Atg16(1-57)pETDUET-1 into BL21 (DE3) E. coli. An overnight culture was used to inoculate $9 \mathrm{~L}$ ZYM-5052 autoinducible media which was grown to $\mathrm{OD}_{600}=0.6-0.7$ and then incubated at $25^{\circ} \mathrm{C}$ for 24 hours. The general overexpression and lysis protocols were followed. The general IMAC protocol was followed using the Ni-sepharose column. The general IMAC washing buffer was used which contained $30 \mathrm{mM}$ imidazole. IMAC elution buffer contained $1 \mathrm{M}$ imidazole. For IMAC purification of His-Atg5 Atg16(157), buffers were supplemented with $1 \mathrm{mM} \mathrm{MgCl}_{2}$. After IMAC, pure fractions were pooled and dialyzed overnight in gel filtration buffer. For His-Atg5 Atg16(1-46) this buffer was comprised of $0.15 \mathrm{M} \mathrm{NaCl}, 20 \mathrm{mM}$ HEPES pH 6.8, $2 \mathrm{mM} \beta$-mercaptoethanol. His-Atg5 Atg16(1-57) gel filtration buffer consisted of $0.15 \mathrm{M} \mathrm{NaCl}, 20 \mathrm{mM}$ Tris $\mathrm{pH} 7.4$, $1 \mathrm{mM} \mathrm{MgCl}_{2}$. The complex was then concentrated loaded onto a HiLoad ${ }^{\mathrm{TM}}$ 16/60 Superdex ${ }^{\text {TM }} 200$ prep grade column.

\subsection{Molecular weight determination}

\subsubsection{Analytical gel filtration}

To determine the size of Atg16L1(53-168) or Atg5 Atg16L1 complexes, size exclusion chromatography with a Superdex ${ }^{\text {TM }} 200$ 10/300 GL column was used. High and low molecular weight standards (Pharmacia Biotech) including aldolase, ovalbumin, ribonuclease A, BSA, thyroglobulin, chymotrypsinogen A, ferritin, catalase, and dextran blue were used to calibrate the column. $500 \mu \mathrm{L}$ of $2 \mathrm{mg} / \mathrm{mL}$ protein in gel filtration buffer 
(200 mM NaCl, $30 \mathrm{mM}$ Hepes pH 7.5, $5 \mathrm{mM} \beta$-mercaptoethanol). $1.25 \mathrm{CV}$ of gel filtration buffer were applied to the column at a flow rate of $0.5 \mathrm{~mL} / \mathrm{min}$ and $0.5 \mathrm{~mL}$ fractions were collected. The gel phase coefficient distribution values, $K_{a v}$, of the Atg5 Atg16L1 complexes and the molecular weight standards were calculated from their elution volumes using the following equation: $\mathrm{K}_{\mathrm{av}}=\left(\mathrm{V}_{\mathrm{e}}-\mathrm{V}_{\mathrm{o}}\right) /\left(\mathrm{V}_{\mathrm{t}}-\mathrm{V}_{\mathrm{o}}\right)$ where $\mathrm{V}_{\mathrm{e}}=$ elution volume of the protein, $\mathrm{V}_{\mathrm{o}}=$ void volume of the column, and $\mathrm{V}_{\mathrm{t}}=$ total column bed volume. The standard curve was generated by plotting the $\mathrm{K}_{\mathrm{av}}$ values on the $\mathrm{y}$ axis and the $\log$ molecular weight values on the $\mathrm{x}$ axis. The equation of the standard curve was $\mathrm{y}=-0.1385(\ln (\mathrm{x}))+1.9561$ with an $\mathrm{R}^{2}=0.9933$. The $\mathrm{R}^{2}$ value determines the goodness of fit of the standard curve where 1 signifies that all data points lie on the standard curve. The molecular weights of the Atg5 Atg16L1 complexes was then determined by inserting the calculated $\mathrm{K}_{\mathrm{av}}$ value into the standard curve equation and solving for $\mathrm{x}$.

\subsubsection{Multi-angle laser light scattering (MALLS)}

To resolve differently sized proteins or different oligomers of a protein or protein complex, $500 \mu \mathrm{L}$ of $2 \mathrm{mg} / \mathrm{mL}$ protein was loaded onto a Superose 6 10/300 GL (GE Healthcare) gel filtration column, which has a separation range from 5 to $500 \mathrm{kDa}$. As the protein was eluted from the column with gel filtration buffer $(0.2 \mathrm{M} \mathrm{NaCl}, 30 \mathrm{mM}$ HEPES $\mathrm{pH} 7.5,100 \mu \mathrm{M}$ tris(2-carboxyethyl)phosphine), it was analyzed by several detectors. The refractive index of the proteins and the UV absorbance at $280 \mathrm{~nm}$ were measured with an Optilab Dsp Interferometric Refractometer (Wyatt Technology). Using this information the concentration of the protein was calculated. The Dawn EOS Enhanced Optical System (Wyatt Technology) was comprised of detectors to record the light scattering signals from multiple angles as the light from the laser was scattered by the protein. Through multiple angle detection, the accuracy of the molecular weight determination was improved for large, elongated, or unfolded proteins since light is no longer scattered uniformly as it is for small, globular proteins. The intensity of the light scattering signal is proportional to the molecular mass of the protein times its concentration. Eclipse software (Wyatt Technology) was used to record the spectra and the Astra program (Wyatt Technology) was used for data analysis and molecular weight determination. 


\subsection{Calpain cleavage and fragment interaction analysis}

\subsubsection{Calpain cleavage of Atg5 and Atg5 Atg16L1 complexes}

Calpain-1 from human erythrocytes (Calbiochem) or recombinant calpain-2 from rat (Calbiochem) was added 1:50 (w/w) to the Atg5 Atg16L1(1-231) or Atg5 Atg16L1(1-70) complex or Atg5 in the presence of calpain assay buffer $(.15 \mathrm{M}$ $\mathrm{NaCl}, 25 \mathrm{mM}$ Tris, $14 \mathrm{mM} \beta$-mercaptoethanol, $5 \mathrm{mM} \mathrm{CaCl}_{2}, \mathrm{pH}$ 7.3). Inhibitors were added to the protein before addition of the calpains. ALLN (Calbiochem, dissolved in DMSO) and ALLM, calpain inhibitor II, (Sigma, dissolved in ethanol) were added to a final concentration of $50 \mu \mathrm{M}$. Acetyl calpastatin(184-210) CS peptide from human (Roche) was added to a final concentration of $10 \mu \mathrm{M}$. Pefabloc SC (Roche) was added to a final concentration of $4 \mathrm{mM}$. Samples taken at time zero were taken before the addition of calpain or inhibitors. The reactions were incubated at $37{ }^{\circ} \mathrm{C}$ (unless otherwise indicated) and $40 \mu \mathrm{L}$ samples were taken at the given time points and combined with 20 $\mu \mathrm{L} 3 \mathrm{x}$ SDS-PAGE sample buffer. Samples were boiled at $95^{\circ} \mathrm{C}$ for 3-5 min.

\subsubsection{Atg5 interaction analysis of calpain cleavage products}

Atg5 Atg16L1(1-231) was digested with calpain-1 1:50 (w/w) as for the calpain cleavage protocol. After 60 minutes of digestion, the reaction was stopped by adding ALLN to a final concentration of $5 \mathrm{mM}$. The sample was then loaded onto a column that had been equilibrated in binding/wash buffer $\left(0.2 \mathrm{M} \mathrm{NaCl}, 20 \mathrm{mM} \mathrm{NaH} \mathrm{PO}_{4}, 20 \mathrm{mM}\right.$ imidazole, $\mathrm{pH}$ 7.4). The reaction was repeatedly loaded 5 times to enhance binding. The column was washed with $5 \mathrm{CV}$ washing buffer and then the protein was eluted with $5 \mathrm{CV}$ elution buffer (0.2 M NaCl, $20 \mathrm{mM} \mathrm{NaH} \mathrm{PO}_{4}, 500 \mathrm{mM}$ imidazole, $\mathrm{pH}$ 7.4). All washing and elution steps were performed manually with syringes.

\subsubsection{Western blot of Atg5 \& Atg5 Atg16L1(1-231) following calpain cleavage}

The Western blotting general protocol was followed (see section 2.2.6). Gel electrophoresis was performed with a $15 \%$ SDS-PAGE gel. The nitrocellulose membrane was then immunoblotted using rabbit anti-Apg5L polyclonal antibody (Proteintech Group, Inc.) as the primary antibody diluted in blocking buffer $1: 1000(\mathrm{v} / \mathrm{v}) 4{ }^{\circ} \mathrm{C}$ overnight. The secondary antibody, goat anti-rabbit ECL Plex Cy5 (GE Healthcare) was applied as a 1:2000 (v/v) dilution in blocking buffer and incubated in the dark for $45 \mathrm{~min}$ at RT with 
shaking. Fluorescence [Cy5] detection was chosen with $25 \mu \mathrm{m}$ pixel size and PMT 600 mode free.

\subsection{Complex formation between Rab33BQ92L and Atg5 Atg16L1 complexes}

\subsubsection{Analytical gel filtration}

To determine whether His-Rab33B(30-202)Q92L interacted with Atg16L1 as part of the Atg5 Atg16L1(1-265) complex, size exclusion chromatography with an analytical Superdex ${ }^{\mathrm{TM}} 200$ 10/300 GL column was performed. $500 \mu \mathrm{L}$ of His-Rab33B(30202)Q92L diluted to $2.5 \mathrm{mg} / \mathrm{mL}$ in gel filtration buffer consisting of $0.4 \mathrm{M} \mathrm{NaCl}, 30 \mathrm{mM}$ Hepes pH 7.5, $2 \mathrm{mM} \mathrm{MgCl} 2,2 \mathrm{mM} \beta$-mercaptoethanol, and $10 \mu \mathrm{M}$ GTP was loaded onto the equilibrated column and eluted with $1.25 \mathrm{CV}$ of gel filtration buffer at a flow rate of $0.5 \mathrm{~mL} / \mathrm{min}$ during which $0.5 \mathrm{~mL}$ fractions were collected. His-Rab33B(30-202)Q92L was added with a 4-four molar excess compared to the Atg5 Atg16L1(1-265) complex (3 $\mathrm{mg} / \mathrm{mL}$ ). $500 \mu \mathrm{L}$ of this mixture was loaded onto the column. For the run of the Atg5 Atg16L1(1-265) complex on its own, the proteins were diluted in gel filtration buffer comprised of $0.15 \mathrm{M} \mathrm{NaCl}, 30 \mathrm{mM}$ HEPES $\mathrm{pH}$ 7.5. This buffer was utilized for reequilibration of the column as well as during the run. Elution fractions were analyzed by SDS-PAGE.

\subsubsection{Isothermal titration calorimetry}

Isothermal titration calorimetry experiments were conducted using a VP-ITC microcalorimeter (Microcal) to measure the thermodynamic parameters of the interaction between Rab33B(30-202)Q92L and Atg16L1 in complex with Atg5. The VP-ITC machine consists of two identical cells (each $\sim 1.4 \mathrm{~mL}$ ) which are surrounded by an adiabatic jacket to prevent heat exchange with the outside. One cell is used as a reference and is filled with water. The other cell was filled with the Atg5 Atg16L1 complex. A constant power was applied to the reference cell to maintain both cells at equal temperature. A specific amount of Rab33B(30-202)Q92L (total volume of the syringe $\sim 300 \mathrm{~mL}$ ) was titrated into a solution of known concentration of Atg5 Atg16L1 in the cell. When the proteins interacted with one another, an exothermic reaction occurred and heat was given off. Therefore less power had to be applied to the sample cell in 
comparison to the reference cell in order to keep both at the same temperature. The amount of heat given off was integrated with respect to time.

VPViewer2000ITC software was used to control the machine. The cell and syringe were cleaned with double-distilled water, 10\% SDS solution, 100\% ethanol and again with water before being washed with buffer. The proteins were dialyzed overnight in buffer $\left(0.2 \mathrm{M} \mathrm{NaCl}, 30 \mathrm{mM}\right.$ HEPES pH 7.5, $1 \mathrm{mM} \mathrm{MgCl}_{2}, 10 \mu \mathrm{M}$ GTP, $2 \mathrm{mM} \beta$ mercaptoethanol) and then dialyzed for 3 to 4 hours in fresh buffer the next morning. This fresh buffer was then used for equilibrating the cell and syringe after cleaning. A control run was performed first where buffer in the syringe was titrated into buffer in the cell to make sure that there was heat given off as an effect of buffer dilution and that the cell and syringe were properly cleaned. All buffers and samples used in the experiments were degassed at $20{ }^{\circ} \mathrm{C}$ for $5 \mathrm{~min}$ since air bubbles show up as heat spikes and interfere with accurate measurement and quantification.

The following settings were used during the experiments: cell temperature $=25^{\circ} \mathrm{C}$, reference power $=10 \mu \mathrm{Cal} / \mathrm{sec}$, initial delay $=100 \mathrm{sec}$, stirring speed $=250 \mathrm{rpm}$, feedback mode/gain=high, fast equilibration $=$ on, and auto equilibration $=$ on, number of injections $=20$, duration $=30 \mathrm{sec}$, filter period $=3 \mathrm{sec}$. The first injection is considered unreliable and was removed from data integration, therefore only $5 \mu \mathrm{L}$ of Rab33B(30202)Q92L with a spacing of 250 seconds was used for this initial titration step. Thereafter $15 \mu \mathrm{L}$ titration steps were performed with 500 seconds spacing in between. The concentration of $\operatorname{Rab33B}(30-202) \mathrm{Q} 92 \mathrm{~L}$ in the cell was $75 \mu \mathrm{M}$. The concentration of either Atg5 Atg16L1(1-265) or Atg5 Atg16L1(1-231) in the cell was $5 \mu \mathrm{M}$.

\subsection{Limited proteolysis and interaction analysis via chromatography}

\subsubsection{Limited proteolysis}

GST-Atg16L1(328-623)

Murine GST-Atg16L1(328-623) was digested with proteases to characterize its stability and identify smaller, stable fragments for crystallization. Either sequencing grade trypsin dissolved in $1 \mathrm{mM} \mathrm{HCl}$ (Roche) or chymotrypsin (Roche) were added to $1 \mathrm{mg} / \mathrm{mL}$ purified GST-Atg16L1(328-623) diluted in $100 \mathrm{mM} \mathrm{NaCl}, 30 \mathrm{mM}$ Hepes pH 7.5, $50 \mathrm{mM}$ Tris- $\mathrm{HCl} \mathrm{pH} 8.0$ in a 1:200 (w/w) ratio. The digestions were incubated at RT. $40 \mu \mathrm{L}$ 
samples were taken before addition of protease and then at indicated time points after protease addition, combined with $3 \mathrm{x}$ loading buffer and immediately boiled at $95{ }^{\circ} \mathrm{C}$ for 3 $\min$.

\section{His-Atg5 Atg16L1(1-231)}

Tryptic digestion was performed using the same ratio as above. Samples for the $15 \%$ SDS-PAGE gel were taken before addition of trypsin and after 45 min incubation at RT. The remaining digested protein was inactivated with $4 \mathrm{mM}$ Pefabloc SC (Roche) a serine protease inhibitor.

\section{His-Atg5 His-Atg16L1(1-265) His-Rab33B(30-202)Q92L}

The Atg5 Atg16L1(1-265) complex was pre-incubated with His-Rab33B(30202)Q92L for $30 \mathrm{~min}$ at $4^{\circ} \mathrm{C}$. The proteins were diluted to a final concentration of $1 \mathrm{mg} / \mathrm{mL}$ in buffer comprised of $0.15 \mathrm{M} \mathrm{NaCl}, 30 \mathrm{mM}$ HEPES $\mathrm{pH}$ 7.5. Trypsin, chymotrypsin, elastase, and proteinase $\mathrm{K}$ were added 1:200 (w/w) to the protein mixture. Reactions containing chymotrypsin, elastase and proteinase $\mathrm{K}$ were adjusted to $50 \mathrm{mM}$ Tris $\mathrm{pH}$ 8.0. The reaction proceeded at RT. Samples were taken after 5, 10, 15, 30, 60, and 120 minutes and combined with 5x Schägger Jagow loading buffer and boiled at 95 ${ }^{\circ} \mathrm{C}$ for 5 min for analysis on a Schägger Jagow gel.

\subsubsection{Interaction analysis of cleavage products via chromatography}

\section{His-Atg5 Atg16L1(1-231)}

After proceeding as described for the complex in section 2.7.1, $1 \mathrm{M}$ imidazole was then added to the reaction to make a final concentration of $20 \mathrm{mM}$ to prevent nonspecific binding to the $1 \mathrm{~mL}$ HisTrap ${ }^{\mathrm{TM}} \mathrm{FF}$ column (GE Healthcare). Washing and elution steps were done manually with a syringe. The column was equilibrated with $5 \mathrm{CV}$ binding/wash buffer (200 mM NaCl, $50 \mathrm{mM}$ Tris-HCl Buffer $\mathrm{pH}$ 8.0, $20 \mathrm{mM}$ imidazole, $\mathrm{pH}$ 8.0). To maximize binding of the protein fragments to the column, the flow-through was reloaded onto the column four times. Next, the column was washed with $5 \mathrm{CV}$ binding/wash buffer of which the first two wash fractions were comprised of $1.5 \mathrm{~mL}$ fractions and third and fourth of $1 \mathrm{~mL}$ fractions. $5 \mathrm{CV}$ elution buffer $(200 \mathrm{mM} \mathrm{NaCl}, 50$ $\mathrm{mM}$ Tris- $\mathrm{HCl} \mathrm{pH} 8.0,500 \mathrm{mM}$ imidazole, $\mathrm{pH} 8.0$ ) were applied to the column and $1 \mathrm{~mL}$ fractions were collected. 


\section{His-Atg5 His-Atg16L1(1-265) His-Rab33B(30-202)Q92L}

The samples were digested with trypsin as described in section 2.7.1 for $60 \mathrm{~min}$ before the reaction was stopped with $4 \mathrm{mM}$ Pefabloc. For anion and cation exchange chromatography, a Mono Q 5/50 GL (GE Healthcare) and a Mono S 5/50 GL (GE Healthcare) column were used. After equilibration with binding buffer (Mono $Q=20 \mathrm{mM}$ Tris $\mathrm{pH}$ 8.0; Mono $\mathrm{S}=20 \mathrm{mM}$ MES $\mathrm{pH}$ 6.0), the digested sample was diluted to $10 \mathrm{~mL}$ with binding buffer and applied to the column. The column was washed with $5 \mathrm{CV}$ (1 $\mathrm{CV}=1 \mathrm{~mL}$ ) and the proteins were eluted with a gradient from $0-100 \%$ elution buffer (binding buffer containing $1 \mathrm{M} \mathrm{NaCl}$ ) over $20 \mathrm{CV}$ during which $1 \mathrm{~mL}$ fractions were collected.

\section{His-Rab33B(30-202)Q92L Atg16L1(141-265)}

The samples were digested with trypsin as described above for $5 \mathrm{~min}$ before the reaction was stopped with $4 \mathrm{mM}$ Pefabloc. For anion and cation exchange chromatography, a Mono Q 5/50 GL (GE Healthcare) and a Mono S 5/50 GL (GE Healthcare) column were used. After equilibration with binding buffer (Mono $Q=20 \mathrm{mM}$ Tris pH 8.0, $2 \mathrm{mM} \mathrm{MgCl}_{2}$; Mono $\mathrm{S}=20 \mathrm{mM}$ MES pH 6.0, $2 \mathrm{mM} \mathrm{MgCl}_{2}$ ), the digested sample was diluted 1:4 with binding buffer and applied to the column. The column was washed with $5 \mathrm{CV}(1 \mathrm{CV}=1 \mathrm{~mL})$ and the proteins were eluted with a gradient from 0 $100 \%$ elution buffer (binding buffer containing $1 \mathrm{M} \mathrm{NaCl}$ ) over $20 \mathrm{CV}$ during which $1 \mathrm{~mL}$ fractions were collected.

\section{His-Atg5 His-Atg16L1(1-106) \pm D-myo-inositol-(1,3)-diphosphate $\left[I P(1,3)_{2}\right]$}

The proteases provided in the Proti-Ace ${ }^{\mathrm{TM}}$ (Hampton Research, HR2-429) and Proti-Ace TM 2 (HR2-432) Kits were first reconstituted in $100 \mu \mathrm{L}$ double-distilled $\mathrm{H}_{2} \mathrm{O}$. The proteases were then diluted $1: 10$ in the dilution buffer provided in the kit. $5 \mu \mathrm{L}$ of the previously diluted $0.1 \mathrm{mg} / \mathrm{mL}$ protease was pipetted into $5 \mu \mathrm{L}$ of the His-Atg5 His-

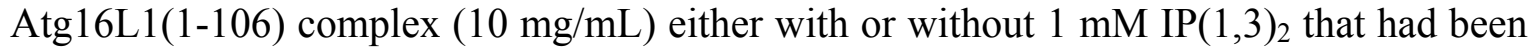
adjusted to $30 \mathrm{mM}$ HEPES pH 7.5 and $0.2 \mathrm{M} \mathrm{NaCl}$. The reaction was then incubated at RT or $37^{\circ} \mathrm{C}$, as indicated, and samples were taken after $5,10,15,20,30$, and 60 minutes and combined with $2 x$ Schägger Jagow loading buffer and boiled for $5 \mathrm{~min}$ at $95^{\circ} \mathrm{C}$. For $\mathrm{N}$-terminal sequencing, the procedure above was followed using clostripain and $\alpha$ chymotrypsin as proteases. The reaction proceeded at RT for 5 and $10 \mathrm{~min}$ and the proteins were resolved on a Schägger Jagow gel. 


\subsubsection{Cleavage site determination via $\mathrm{N}$-terminal protein sequencing}

The protocol given by Seqlab GmbH (Goettingen, Germany) was followed. The sample was resolved by gel electrophoresis using an SDS-PAGE gel. The gel, Immobilon-FL Transfer PVDF membrane (Millipore), and 4 sheets of Whatman gel blotting filter paper (Whatman) were gently shaken in CAPS buffer. A semi-dry blotting apparatus (Phase, Lübeck) (Towbin 1979, 1989) was used: first two sheets of filter paper were placed on the bottom half of the apparatus. The gel was placed on top of this and the PVDF was placed on top of the gel. Two more sheets of filter paper completed the sandwich and bubbled were removed by gently rolling a pipet on top. The device was run at 45 to $50 \mathrm{~mA}$ for 20 to 45 minutes causing the negatively charged SDS-coated proteins to move upward toward the positive electrode and thus be transferred onto the membrane. The blot was stained less than 1 min with the Coomassie Brilliant Blue because the acetic acid can interfere with sequencing. The membrane was allowed to dry overnight before the band was cut out and placed in a polypropylene cut for shipping.

\subsection{Crystallization and structure determination}

\subsubsection{Crystallization screen setup}

\section{6 well MRC or Greiner sitting drop plates}

Crystallization trials were set up using 96 well sitting drop plates that were comprised of 3 round drops/well Greiner (Hampton Research) or 2 round drops/well (MRC, Hampton Research) were pipetted using the Cartesian Microsys (Cartesian Dispensing Systems) nanodispenser robot. $100 \mathrm{~nL}$ of protein was pipetted into the sitting drop well followed by $100 \mathrm{~nL}$ of the reservoir solution (if the volume varies, it is indicated in the tables). Screens that produced crystals included the following condition suites from Qiagen: Classics, ClassicsII, Classics Lite, Cryos, JCSG+, Protein Complex, PEGs, PEGsII, PACT, ComPAS, Cations, MbClass I, MbClass II, pHClear, pH ClearII, and Nucleix. The SaltRx and Index screens from Hampton as well as the Wizard I\&II and Wizard III\&IV screens from Emerald Biosystems.

After pipetting, the screen was carefully sealed with clear sealing tape and stored at either $20{ }^{\circ} \mathrm{C}$ or $4{ }^{\circ} \mathrm{C}$ as indicated in an automated robotic Formulatrix crystallization imager which uses RockImager (C2008 Formulatrix) software to take images of the drops 
at regular intervals. Rockmaker Main Application software (C2008 Formulatrix) was used to view images.

\section{4-well Linbro hanging drop plates}

Crystallization conditions were optimized Linbro 24-well crystallization plates (Jena Bioscience) using the hanging drop method where $1 \mu \mathrm{L}$ of protein was pipetted onto a circular, $22 \mathrm{~mm}$ siliconized cover slide and $1 \mu \mathrm{L}$ of reservoir was pipetted onto the side of the protein drop (if the amount varies it is indicated in the tables). 1 to 3 drops were pipetted per cover slide. After the protein and reservoir was pipetted, the cover slide was flipped upside down and placed onto a well that was greased with Bayer medium viscosity silicone grease (Jena Bioscience) and that contained $1 \mathrm{~mL}$ of reservoir solution. All chemicals used in the optimization screens were from Sigma, Fluka, or Sigma-Aldrich and were analytical grade with $\geq 99.0 \%$ purity.

\section{Additive screens set up}

For additive screens using 96-well MRC or Greiner sitting drop plates, the Cartesian robot was utilized to pipet first $100 \mathrm{~nL}$ of protein into each drop in each well, followed by $100 \mathrm{~nL}$ reservoir, and finally $20 \mathrm{~nL}$ additive. Alternatively a program could be used that pipetted in $200 \mathrm{~nL}, 200 \mathrm{~nL}$, and $40 \mathrm{~nL}$ of protein, reservoir, and additive, respectively.

When 24-well Linbro plates were used with the hanging drop method, $1.25 \mu \mathrm{L}$ of protein was first pipetted onto the circular, $22 \mathrm{~mm}$ siliconized cover slide followed by 1 $\mu \mathrm{L}$ reservoir solution added to the protein and finally $0.25 \mu \mathrm{L}$ additive added to the drop on the cover slide. In addition, the rate of vapour diffusion was sometimes lowered by adding a layer of Al's oil $(250-500 \mu \mathrm{L})$ onto the reservoir solution before placing the cover slide with drops onto the well when the hanging drop method was employed (Chayen 1997).

\section{Streak seeding setup}

Streak seeding is a technique used to grow larger crystals. Here, an acupuncture needle was used to crush a crystal. The needle was then streaked once through a fresh drop of protein combined with reservoir solution. In this way, the very small pieces of the crystal introduced in the protein solution serving as seeds to promote crystal growth. 


\subsubsection{Flash cooling of the crystals}

Crystals were equilibrated in cryoprotectant before being flash-cooled in liquid nitrogen to prevent ice formation. Typically a serial dilution of the cryoprotectant was performed whereby the crystal was first transferred to a drop of reservoir solution (mother liquor). The crystal was then fished out of this drop and transferred to a drop containing equal parts mother liquor and cryoprotectant. After equilibration in this solution varying from 30 seconds to several minutes, the crystals were transferred to a drop of cryoprotectant (mother liquor supplemented with a cryoprotective compound). After equilibration in cryoprotectant for 30 seconds to several minutes, the crystal was fished out of the drop and flash-cooled in liquid nitrogen. Cryoprotectants used contained glycerol, ethylene glycol, xylitol and sucrose, and PEG 400.

\subsubsection{X-ray diffraction data collection and processing}

Diffraction data of cryo-cooled crystals were collected at $100 \mathrm{~K}$ on a Pilatus detector at the Swiss Light Source (Switzerland) beamline PXII. The program package $X D S$ (Kabsch 1988, 1993, 2010a,b) which is a 3-part program consisting of $X D S$, $X S C A L E$, and $X D S C O N V$ was utilized for processing of the $\mathrm{x}$-ray diffraction data.

$X D S$, through its eight successive subroutines, was used to process a single dataset and produce a list of corrected, integrated intensities. First, XYCORR calculates the spatial corrections at each detector pixel. INIT then determines three tables based on the noise due solely to the detector, the variation in the pixel contents in the background region, and the estimation of the initial background. These tables are then used in later steps to determine whether a pixel is classified as a diffraction spot or as background. The subroutine COLSPOT searches through a subset of images and locates the strong diffraction spots to be used for indexing in the ensuing IDXREF subroutine. During indexing, the orientation, dimensions, and symmetry of the crystal lattice are determined. DEFPIX creates a table of initial background pixels that it has marked as untrusted either due to their being obscured by hardware like the beam stop or because they were outside the user-defined resolution range. XPLAN can be used to develop an optimized data collection strategy for a number of resolution shells. The INTEGRATE subroutine calculates the intensity of each reflection from all images. If the space group is still unknown, it will be automatically determined in the final CORRECT step. In this subroutine, correction factors are also applied to the measured intensities, the unit cell parameters are refined, and the statistics of data completeness and quality are reported. 
$X S C A L E$ puts one or more datasets, which were processed with $X D S$ on a common scale. Symmetry related reflections can be merged and the overall completeness and quality of the data is reported. A resolution cut off for the data was chosen such that the signal to noise ratio $(\mathrm{I} / \sigma(\mathrm{I}))$ was $\geq 3$ and the $\mathrm{R}$ factor which describes the consistency of intensities between symmetry-related reflections (Diederichs and Karplus 1997) should be $\leq 40 \%$.

$X D S C O N V$ is then used to convert the XSCALE output into other data formats needed for software packages used for structure determination. Measured intensities were converted into structure factor amplitudes in the mtz format needed for the CCP4 suite (Collaborative Computational Project Number 4 1994, Potterton 2003). During this step, five percent of the reflections were randomly chosen and put aside to form a test set of reflections which would remain unbiased during refinement for the calculation of $\mathrm{R}_{\text {free }}$.

\subsubsection{Calculation of the Matthews coefficient and the self-rotation function}

The Matthews coefficient, $\mathrm{V}_{\mathrm{m}}$, (Matthews 1968, 1976) was determined using the Matthews coefficient program that is part of the CCP4 suite (Collaborative Computational Project Number 4 1994, Potterton 2003). It is defined as the ratio of the crystal volume per unit of molecular weight of the protein. This value is used to estimate the number of molecules present in the asymmetric unit since the solvent should occupy between 40 to $60 \%$ of the total volume with the lower and upper limits for solvent content being $27 \%$ and $78 \%$, respectively. The range of $\mathrm{V}_{\mathrm{m}}$ usually falls between $2.3 \AA^{3} / \mathrm{Da}$ and $2.7 \AA^{3} / \mathrm{Da}$ (Kantardjieff 2003).

The self-rotation function shows the presence of all crystallographic and noncrystallographic rotational symmetry axes and their orientations to each other. MOLREP (Vagin 1997), which is part of the CCP4 suite (Collaborative Computational Project Number 4 1994, Potterton 2003) was used for calculation of the self-rotation function. To perform a self-rotation function calculation, two Patterson maps are calculated from the measured structure factors. One is kept in a constant orientation whereas the other is rotated in small increments. Both maps are overlaid and integrated. At certain angles which correspond to rotational symmetry elements both maps will coincide resulting in a peak in the self-rotation function.

Results are visualized by stereographic projections. Peaks at the section chi $=180^{\circ}$ indicate the presence of two-fold crystallographic and/or non-crystallographic symmetry 
axes, peaks at the section chi $=120^{\circ}$ show three-fold symmetry axes, at chi $=90^{\circ}$ four-fold symmetry axes and so on.

\subsubsection{Molecular replacement}

For the calculation of the electron density map both structure factors and phases are needed.

$$
\rho(x \text { y z })=1 / V \sum_{h k l}|F(h k l)| e^{[-2 \pi i(h x+k y+l z)+i \alpha(h k l)]}
$$

The structure factor amplitudes are derived directly from the measured intensities, whereas the phases cannot be determined from the experimental data. However, if the structure of a homologue with a sequence identity of $\sim 30 \%$ or higher is known, molecular replacement can be used to solve the phase problem. We used the MOLREP program (Vagin 1997) which performed first a rotational search and then a translational search to identify the positions of the molecules in the unit cell. For that purpose Patterson functions are calculated from both the measured structure factors and the model.

The Patterson function is a Fourier summation with the squared structure factors as coefficients and without phase angles.

$$
\mathrm{P}(\mathrm{u} \mathrm{v} \mathrm{w})=1 / \mathrm{V} \sum_{\mathrm{hkl}}|\mathrm{F}(\mathrm{hkl})|^{2} \cos [2 \pi(\mathrm{hu}+\mathrm{kv}+\mathrm{lw})]
$$

The Patterson function is a map of vectors between pairs of atoms in a structure. If the vectors are between two atoms within the same molecule, called self-Patterson vectors, they are generally shorter compared to vectors between pairs of atoms from different molecules, called cross-Patterson vectors.

During the rotational search, the model is rotated in small increments and the corresponding intramolecular self-Patterson vectors are calculated and compared with the Patterson function calculated from the experimental data. If both Patterson functions match, the rotational parameters have been found. In the next step a translational search is performed to look for a match between the calculated cross-Patterson vectors for the model with unaccounted-for peaks in the experimental Patterson function. Once the location of the molecules in the unit cell has been identified, the phases can be derived from their positions to calculate the electron density with the measured structure factors.

In this study, the published yeast Atg5 Atg16(1-46) (PDB ID: 2DYM) and Atg5 Atg16(1-57) (PDB ID: 2DYO) structures were used as models for molecular 
replacement (Matsushita 2007). Since we used the same constructs in our study, the side chains were not deleted and these structures were used as models for molecular replacement.

\subsubsection{Refinement, density visualization, and generation of figures}

REFMAC5 (Murshudov 1997) was used for refinement. Refinement is a process where the agreement of an atomic model with the observed diffraction data is optimized with the consideration of known stereochemical restraints. The calculated structure factors $\left(\mathrm{F}_{\text {calc }}\right)$ derived from the model are compared with the experimentally measured structure factors $\left(\mathrm{F}_{\mathrm{obs}}\right)$. This yields an R-factor which is used assess the quality of the model. The $\mathrm{R}$-factor for a well-refined structure usually lies in the range of $15-25 \%$ depending on the model completeness, the resolution and the quality of the data. REFMAC5 was used to perform an initial cycle of rigid body refinement followed by restrained refinement. After refinement, both the $2 \mathrm{mF}_{\mathrm{o}}-\mathrm{DF}_{\mathrm{c}}$ and $\mathrm{mF}_{\mathrm{o}}-\mathrm{DF}_{\mathrm{c}}$ electron density maps were calculated with REFMAC5. The electron density maps and models were inspected with COOT (Emsley 2004, 2010) which was also used for model building to include the N-terminal residues of Atg5 in the yeast Atg5 Atg16(1-57) structure, which was then followed by a cycle of restrained refinement with REFMAC5. Figures were generated using the software $P Y M O L$ (Delano, http://www.pymol.org).

\subsection{Characterization of lipid-protein interactions}

\subsubsection{Protein lipid overlay assay}

Protein lipid overlay assays (Dowler 2002) were performed using Membrane Lipid Strips, PIP strips, and PIP arrays (Echelon Biosciences Inc.). For mammalian Atg5 Atg16L1, phosphate buffered saline (PBS) (137 mM NaCl, $2.7 \mathrm{mM} \mathrm{KCl}, 10.1 \mathrm{mM}$ $\mathrm{Na}_{2} \mathrm{HPO}_{4}, 1.8 \mathrm{mM} \mathrm{KH} \mathrm{PO}_{4}, 0.5 \mathrm{mM} \mathrm{MgCl}$, adjusted to $\mathrm{pH} 7.4$ ) was used. For yeast Atg5 Atg16 complexes, the buffer was comprised of $150 \mathrm{mM} \mathrm{NaCl}, 20 \mathrm{mM} \mathrm{Na} \mathrm{HPO}_{4}$, $0.5 \mathrm{mM} \mathrm{MgCl}$, adjusted to $\mathrm{pH} 7.3$. The washing buffer was composed of $0.1 \%(\mathrm{v} / \mathrm{v})$ Tween-20 in PBS. The blocking buffer was $1 \%$ milk powder in PBS. The plastic boxes containing the strips were covered in aluminum foil and were gently shaken during all incubation steps. 
The membrane was first blocked with blocking buffer for 1 hour at RT. The Atg5 Atg16(L1) complexes were then diluted into blocking buffer (final concentration= 5 $\mu \mathrm{g} / \mathrm{mL}$ for mammalian complexes, $20 \mu \mathrm{g} / \mathrm{mL}$ for yeast complexes, and $500 \mu \mathrm{g} / \mathrm{mL}$ for the Atg16L1(1-25) synthesized peptide) and incubated at RT for 1 hour or at $4{ }^{\circ} \mathrm{C}$ overnight. Afterwards the membrane was washed three times with washing buffer for 5-10 min and then incubated with the primary antibody diluted in the blocking buffer for 1 hour at RT. Primary antibodies used were the monoclonal murine anti-hexahistidine-tag (Dianova, DIA 900) at 1:200, murine anti-Strep-tag ${ }^{\circledR}$ II (IBA GmbH, 2-1507-001) at 1:1000, or murine QIAexpress ${ }^{\circledR}$ anti-pentahistidine horseradish peroxidase (HRP) conjugate (Qiagen, 34460) at 1:2000. The membrane was then washed three times as described above before being incubated with goat anti-murine HRP at 1:2000 (Pierce, 31430) for 1 hour at RT. When the QIAexpress ${ }^{\circledR}$ anti-pentahistidine HRP conjugate was used for the primary antibody, no secondary antibody was required. The membrane was washed again as described above. The membrane was covered with Western Lightning ${ }^{\mathrm{TM}}$ Plus-ECL Oxidizing Reagent Plus 1:1 with Western Lightning ${ }^{\mathrm{TM}}$ Plus-ECL Luminol Reagent Plus (Perkin Elmer LAS) and shaken gently for 1 min before being recorded with an Imageready LAS-1000 CCD camera (Fujifilm). Aida software (Fujifilm) was used to visualize the images and export them into other file formats.

\subsubsection{Synthesized Atg16L1(1-25)-StrepII peptide}

\section{Reconstitution of peptide}

The synthesized peptide comprising the first 25 residues of murine Atg16L1 with a linker and StrepII-tag (Biosyntan), MSSGLRAADFPRWKRHIAEELRRRRDSAWSHPQFEK, was sent lyophilized with TFA salts. For buffer exchange into $150 \mathrm{mM}$ $\mathrm{KCl}$ and $20 \mathrm{mM}$ HEPES pH 7.4, a PD MidiTrap G-10 desalting column (GE Healthcare) was used. The column was equilibrated with buffer. $3 \mathrm{mg}$ of peptide was resuspended in $500 \mu \mathrm{L}$ buffer and added to the column. $1.2 \mathrm{~mL}$ buffer was added to the column after the sample had entered the bed. The flow-through was discarded. The peptide was eluted with $1.2 \mathrm{~mL}$ buffer and collected in six fractions. $2 \mu \mathrm{L}$ of each fraction was combined with $50 \mu \mathrm{L}$ of working Bradford solution (see section 2.2.5 Measurement of protein concentration) to see if it contained the peptide. 


\section{Dot blot assay}

$2 \mu \mathrm{L}$ of the each dilution of the peptide $(50,100,150,300,450,600,750$, and 900 $\mu \mathrm{g} / \mathrm{mL}$ ) was spotted onto a piece of nitrocellulose membrane (Whatman, Schleicher and Schuell) and allowed to dry. The same buffers were used as for the mammalian Atg5 Atg16L1 complex in the protein lipid overlay assay (see section 2.9.1) except that the blocking buffer also contained $0.1 \%$ Tween-20 and the buffers did not contain magnesium chloride. All incubation and washing steps occurred with gentle shaking. The membrane was blocked for 15-30 min at RT, then incubated with murine anti-Strep-tag ${ }^{\circledR}$ II (IBA GmbH, 2-1507-001) antibody 1:1000 in blocking buffer overnight at $4^{\circ} \mathrm{C}$. The membrane was washed three times for 5 min each with blocking buffer. The membrane was then incubated in blocking buffer containing 1:2000 goat anti-murine antibody conjugated to HRP (Pierce) for 4 hours at RT. The membrane was washed in blocking buffer once for $15 \mathrm{~min}$ then 2 for $5 \mathrm{~min}$ followed by $5 \mathrm{~min}$ with PBS. The membrane was covered with Western Lightning ${ }^{\mathrm{TM}}$ Plus-ECL Oxidizing Reagent Plus 1:1 with Western Lightning ${ }^{\mathrm{TM}}$ Plus-ECL Luminol Reagent Plus (Perkin Elmer LAS) and shaken gently for 1 min before being recorded with an Imageready LAS-1000 CCD camera (Fujifilm).

\subsubsection{Preparation of liposomes}

All of the lipids were in powder form and reconstituted in chloroform. The lipids used are summarized in Table 2.8. First the reconstituted lipids were pipetted into a round bottom flask with a total lipid amount of $1 \mathrm{mg}$ and the individual components being calculated as $(\mathrm{w} / \mathrm{w})$ percentages. The chloroform was evaporated off using a rotary evaporator (Büchi Rotovapor R-124) [pressure set to lowest possible], for 20-30 min spinning at $\sim 200 \mathrm{rpm}$ which created a lipid film on the bottom of the flask. $50 \mu \mathrm{L}$ buffer containing $150 \mathrm{mM} \mathrm{KCl}, 20 \mathrm{mM}$ HEPES $\mathrm{pH}$ 7.4, 5\% cholate, $2 \mathrm{mM}$ DTT was added to the lipid film to resuspend the lipids. 3 glass beads were added to the flask to aid in the process of bringing the lipids into solution. This was removed and placed into an Eppendorf tube and $100 \mu \mathrm{L}$ of buffer containing $150 \mathrm{mM} \mathrm{KCl}, 20 \mathrm{mM}$ HEPES pH 7.4, $1.5 \%$ cholate, $2 \mathrm{mM}$ DTT was added to the flask to resuspend any remaining lipids. This rinse was then removed and mixed with the previous $50 \mu \mathrm{L}$ of higher detergent containing buffer.

Since cholate forms very small micelles, they can be removed via gel filtration. This gradual removal of detergent from the solubilized lipids causes them to form small unilamellar vesicles (SUVs) which are larger than the micelles and elute from the column 
earlier allowing for their complete separation. These liposomes are approximately $30 \mathrm{~nm}$ in diameter. For size exclusion chromatography, columns which were $0.7 \mathrm{~cm}$ in diameter by $15 \mathrm{~cm}$ in length were used. The matrix was composed $0.5 \mathrm{~g}$ of Sephadex G-50 Superfine beads (Sigma, G5050) suspended in $\sim 10 \mathrm{~mL}$ buffer containing $150 \mathrm{mM} \mathrm{KCl}, 20$ $\mathrm{mM}$ HEPES $\mathrm{pH}$ 7.4. The fractions containing liposomes were identified visually based on the Oregon Green-PE incorporated into the liposomes. The final volume of the pooled fractions was $400-500 \mu \mathrm{L}$. These liposomes were stored at $4{ }^{\circ} \mathrm{C}$.

Table 2.8: Natural and synthetic lipids used for liposome preparation

\begin{tabular}{|c|c|c|c|c|}
\hline Abbreviation & Full name & Source & Company & Cat. \# \\
\hline $\mathrm{PI}(3) \mathrm{P}$ & D-myo-Phosphatidylinositol-3-phosphate & Synthetic & Echelon & P-3016 \\
\hline $\mathrm{PI}(4,5) \mathrm{P} 2$ & L- $\alpha$-phosphatidylinositol-4,5-bisphosphate & Brain, Porcine & Avanti & $840046 \mathrm{P}$ \\
\hline $\begin{array}{l}\text { Oregon } \\
\text { Green-PE }\end{array}$ & $\begin{array}{l}\text { Oregon Green }{ }^{\circledR} 488 \text { 1,2-dihexadecanoyl- } \\
\text { sn-glycero-3-phosphoethanolamine }\end{array}$ & Synthetic & Invitrogen & O-12650 \\
\hline $\begin{array}{l}\text { Texas Red- } \\
\text { PE }\end{array}$ & $\begin{array}{l}\text { Texas Red } \AA \text { 1,2-dihexadecanoyl-sn- } \\
\text { glycero-3-phosphoethanolamine, } \\
\text { triethylammonium salt }\end{array}$ & Synthetic & Invitrogen & $\begin{array}{c}\mathrm{T}- \\
1395 \mathrm{MP}\end{array}$ \\
\hline PE & L- $\alpha$-phosphatidylethanolamine & Brain, Porcine & Avanti & $840022 \mathrm{P}$ \\
\hline $\mathrm{PC}$ & L- $\alpha$-phosphatidylcholine & Brain, Porcine & Avanti & $840053 \mathrm{P}$ \\
\hline DOPC & 1,2-dioleoyl-sn-glycero-3-phosphocholine & Synthetic & Avanti & $850375 \mathrm{P}$ \\
\hline PS & L- $\alpha$-phosphatidylserine & Brain, Porcine & Avanti & $840032 \mathrm{P}$ \\
\hline PI & L- $\alpha$-phosphatidylinositol & Liver, Bovine & Avanti & $840042 \mathrm{P}$ \\
\hline SM & Sphingomyelin & Brain, Porcine & Avanti & $860062 \mathrm{P}$ \\
\hline
\end{tabular}

\subsubsection{Flotation assay}

The flotation assay used is based on work of Matsuoka et al. (1998). In this study a Nycodenz gradient was used with the following additional modifications: $45 \mu \mathrm{L}$ of the liposomes (see above) were combined with $5 \mu \mathrm{L}$ of the Atg5 Atg16 complex $(1.5 \mu \mathrm{M}$ concentration) and incubated at RT for $15 \mathrm{~min}$. This was combined with $50 \mu \mathrm{L}$ of $80 \%$ Nycodenz (Axis-Shield) solution containing $150 \mathrm{mM} \mathrm{KCl,} 20 \mathrm{mM}$ HEPES pH 7.4 and the mixture was thoroughly pipetted until homogenous. This was pipetted into the bottom of an ultracentrifuge tube (Beckman, $7 \times 20 \mathrm{~mm}$ PC tube). $50 \mu \mathrm{L}$ of $30 \%$ Nycodenz solution containing $150 \mathrm{mM} \mathrm{KCl}, 20 \mathrm{mM}$ HEPES pH 7.4 was carefully pipetted on top of the protein/Nycodenz mixture to avoid mixing of the two layers. A layer consisting of $30 \mu \mathrm{L}$ of buffer containing $150 \mathrm{mM} \mathrm{KCl}, 20 \mathrm{mM}$ HEPES pH 7.4 was finally placed on the top. The tubes were spun in an ultracentrifuge (Sorvall Discovery M150SE, Hitachi) at 55000 rpm for $1.5-4$ hours at $4{ }^{\circ} \mathrm{C}$. 
During this centrifugation the less dense liposomes would slowly float up the Nycodenz gradient to the top where there was only buffer. If the protein complex interacted with the liposomes, they would also float up to the upper fractions of the tube. If no interaction took place, the liposomes would float up alone and the protein would remain at the bottom. After centrifugation fractions were taken from the top of the tube to the bottom to analyze whether or not co-flotation of the liposomes and protein had occurred. 20-40 $\mu \mathrm{L}$ fractions were taken and combined with $3 \mathrm{x}$ sample buffer and boiled at $95{ }^{\circ} \mathrm{C}$ for $5 \mathrm{~min}$. The protein was detected by immunoblotting.

\section{Immunoblotting after flotation assays}

The Western blotting general protocol was performed with the following alterations. After electrophoresis, the SDS-PAGE gel and the Whatman filter paper were directly equilibrated in transfer buffer for 5-10 min. The Immobilon-FL Transfer PVDF membrane (Millipore) was hydrated in methanol for 15 seconds then washed with transfer buffer for $10 \mathrm{~min}$. The membrane was blocked in blocking buffer for twice for 10 minutes each. The membrane was immunoblotted with murine anti-hexahistidine (Dianova) monoclonal primary antibody diluted 1:1000 in blocking buffer overnight at $4{ }^{\circ} \mathrm{C}$. Unbound primary antibody was washed off with blocking buffer twice for $10 \mathrm{~min}$ each. Incubation with goat anti-murine HRP conjugated (Pierce) secondary antibody diluted 1:4000 in blocking buffer occurred for 2-4 hours at RT. Unbound secondary antibody was washed off by washing with PBS three times. 


\section{RESULTS}

\subsection{Characterization of mammalian Atg5 Atg16L1 complexes}

\subsubsection{Purification of Atg5 Atg16L1 complexes}

It was first attempted to purify human Atg5 on its own. Full-length Atg5 was tagged with hexahistidine (His) at its N-terminus with the pETDuet-1 plasmid. Rosetta 2 (DE3) E. coli were grown in ZYM-5052 autoinducible media at $37^{\circ} \mathrm{C}$. After they reached $\log$ phase, the cells were cooled on ice for $30 \mathrm{~min}$ before being incubated at $18{ }^{\circ} \mathrm{C}$ for 24 hours and harvested. Since Atg5 was His-tagged, immobilized metal affinity chromatography (IMAC) with Nickel (Ni)-sepharose beads was chosen as the first purification step. The elution fractions contained Atg5 but were still highly impure (Figure 3.1 panel A). The majority of Atg5 was in the insoluble pellet fraction (boxed region of Figure 3.1 panel B). To further purify Atg5, anion exchange chromatography was carried out. The sample was dialyzed in a low salt buffer and applied onto a Mono Q 5/50 GL column (Figure 3.2) and resulted in less than $0.5 \mathrm{mg}$ protein per liter culture.

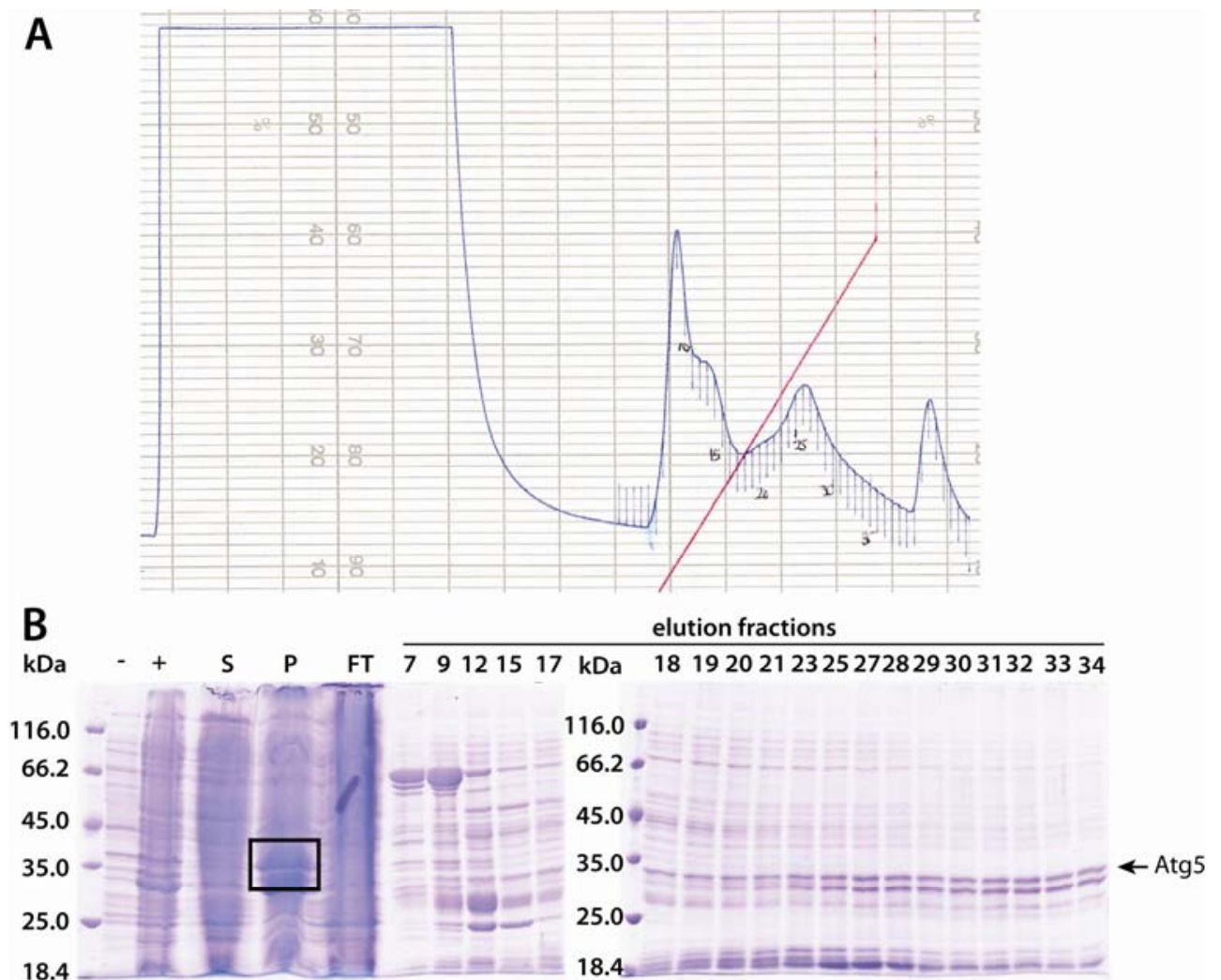

Figure 3.1: Ni-sepharose column purification of full-length human His-Atg5. (A) Chromatogram and (B) $12 \%$ SDS-PAGE gel showing most of the protein is insoluble in the pellet fraction (box). - = uninduced, $+=$ induced, $\mathrm{S}=$ supernatant, $\mathrm{P}=$ pellet, $\mathrm{FT}=$ flow-through, blue line $=\mathrm{UV}_{280}$ curve, red line $=\%$ elution buffer. 
A

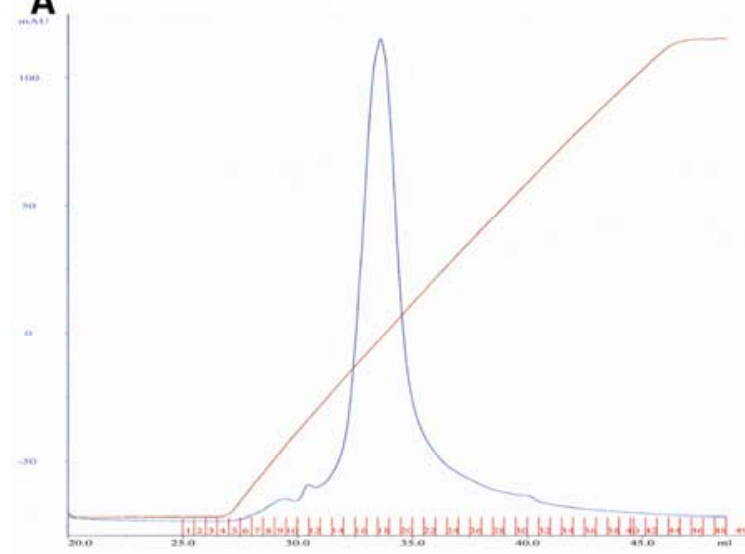

B

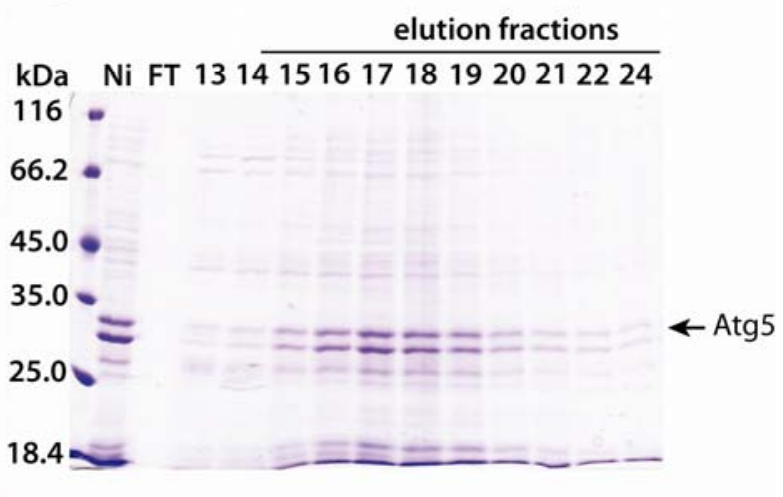

Figure 3.2: Anion exchange chromatography of Atg5. (A) Chromatogram using a Mono Q 5/50 GL column and (B) 12\% SDS-PAGE gel of impure His-Atg5 after anion exchange chromatography. $\mathrm{Ni}=$ sample of pooled fractions after $\mathrm{Ni}$-sepharose column purification, blue line $=\mathrm{UV}_{280}$ curve, brown line $=$ conductivity curve.

Matsushita et al. (2006) showed that full-length yeast Atg5 could be co-purified with the N-terminal Atg5-binding domain (residues 1-46) of yeast Atg16. Since Atg16L interacts with Atg5 in murine cells (Mizushima 2003), the co-expression of human Atg5 with murine Atg16L1 was tried. Murine Atg16L1 was chosen because the full-length cDNA for human Atg16L was not available at the time when this study began. However, since murine Atg16L1 isoform $\gamma$ (the longest isoform) is highly similar to human Atg16L1 with both proteins sharing 94\% sequence identity, a substitution of murine Atg16L1 for the human homologue is feasible.

Multimerization of the Atg12-Atg5 Atg16L1 complex depends on the homooligomerization of Atg16L1 (Mizushima 1999, 2003). Mammalian Atg16L1 differs from yeast Atg16 in that it has an additional WD40 repeat domain at its C-terminus (Mizushima 2003). Atg5 in multiple cloning site 1 (MSC1) of the pETDuet-1 plasmid (ampicillin resistance) was co-expressed in E. coli with full-length murine Atg16L1 as well as truncated murine Atg16L1 constructs using the pET-28a plasmid (kanamycin resistance) (Figure 3.3). Only Atg16L1(1-70) and (1-231) were co-purified with human Atg5. The other murine Atg16L1 constructs were co-purified with murine Atg5 which included a thrombin cleavage site after the His-tag. Rosetta 2 (DE3) E. coli containing both the pET28a and pETDUET-1 plasmids were grown in ZYM-5052 autoinducible media at $37^{\circ} \mathrm{C}$. After they reached $\log$ phase at $37^{\circ} \mathrm{C}$, the cells were cooled on ice for $30 \mathrm{~min}$ before being incubated at $18^{\circ} \mathrm{C}$ for 24 hours and harvested.

The C-terminal Atg16L1 WD40 repeat domain was expressed alone as a GST fusion protein using the pGEX-4T-1 plasmid. GST-Atg16L1(328-623)-pGEX-4T-1- 
containing BL21 (DE3) E. coli were grown in TB medium at $37{ }^{\circ} \mathrm{C}$. Expression was induced with $1 \mathrm{mM}$ IPTG after the cells reached log phase, and the cells were incubated an additional 3.5 hours at $37^{\circ} \mathrm{C}$ before being harvested.

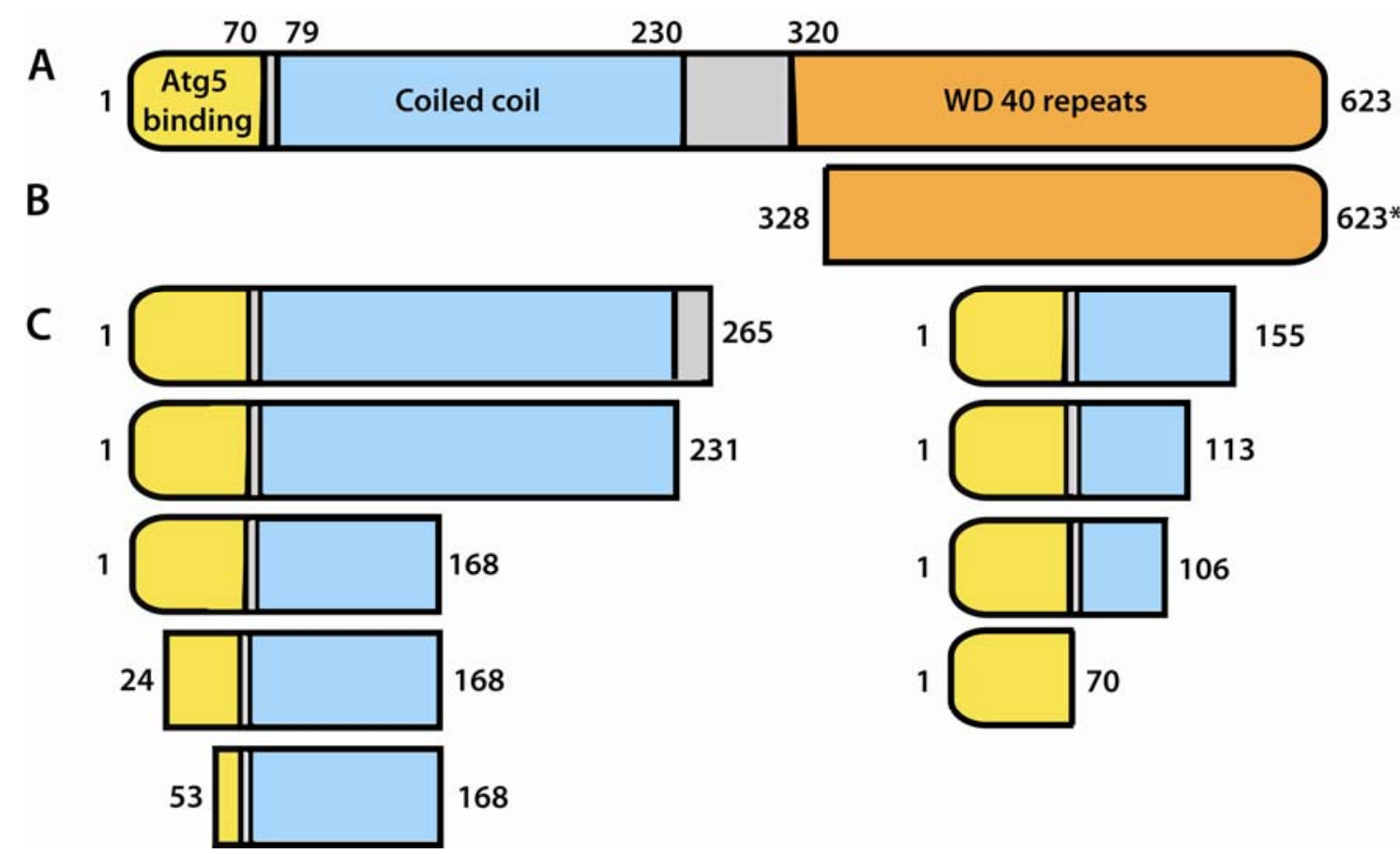

Figure 3.3: Constructs of mammalian Atg16L1 for co-purification with full-length mammalian Atg5. Scheme of (A) full-length murine Atg16L1 comprised of an N-terminal Atg5-binding domain, a central coiled coil domain and a C-terminal WD40 repeat domain co-expressed with Atg5, (B) truncated GST-Atg16L1 fusion protein purified on its own, and (C) truncated Atg16L1 constructs co-expressed with full-length human or murine Atg5.

All of the Atg16L1 constructs co-purified with Atg5 were N-terminally His-tagged proteins and therefore purified first by IMAC and then size exclusion chromatography. His-Atg5 His-Atg16L1(1-70) is the minimal complex lacking the coiled coil domain of Atg16L1. The His-Atg5 His-Atg16L1(1-265) complex comprised the complete coiled coil domain plus an extension of the C-terminal linker region (Figure 3.3). Initial purification by IMAC of His-Atg5 His-Atg16L1(1-70) and His-Atg5 His-Atg16L1(1265) are shown in Figures 3.4 and 3.5, respectively.

The protein complexes were already highly pure after IMAC and required only an additional polishing purification step with size exclusion chromatography (Figures 3.6 and 3.7.) to separate any aggregated protein. Between IMAC and size exclusion chromatography, some of the complexes as denoted in Figures 3.6 and 3.7 had their Histags cleaved by digestion with thrombin during overnight dialysis in gel filtration buffer. 


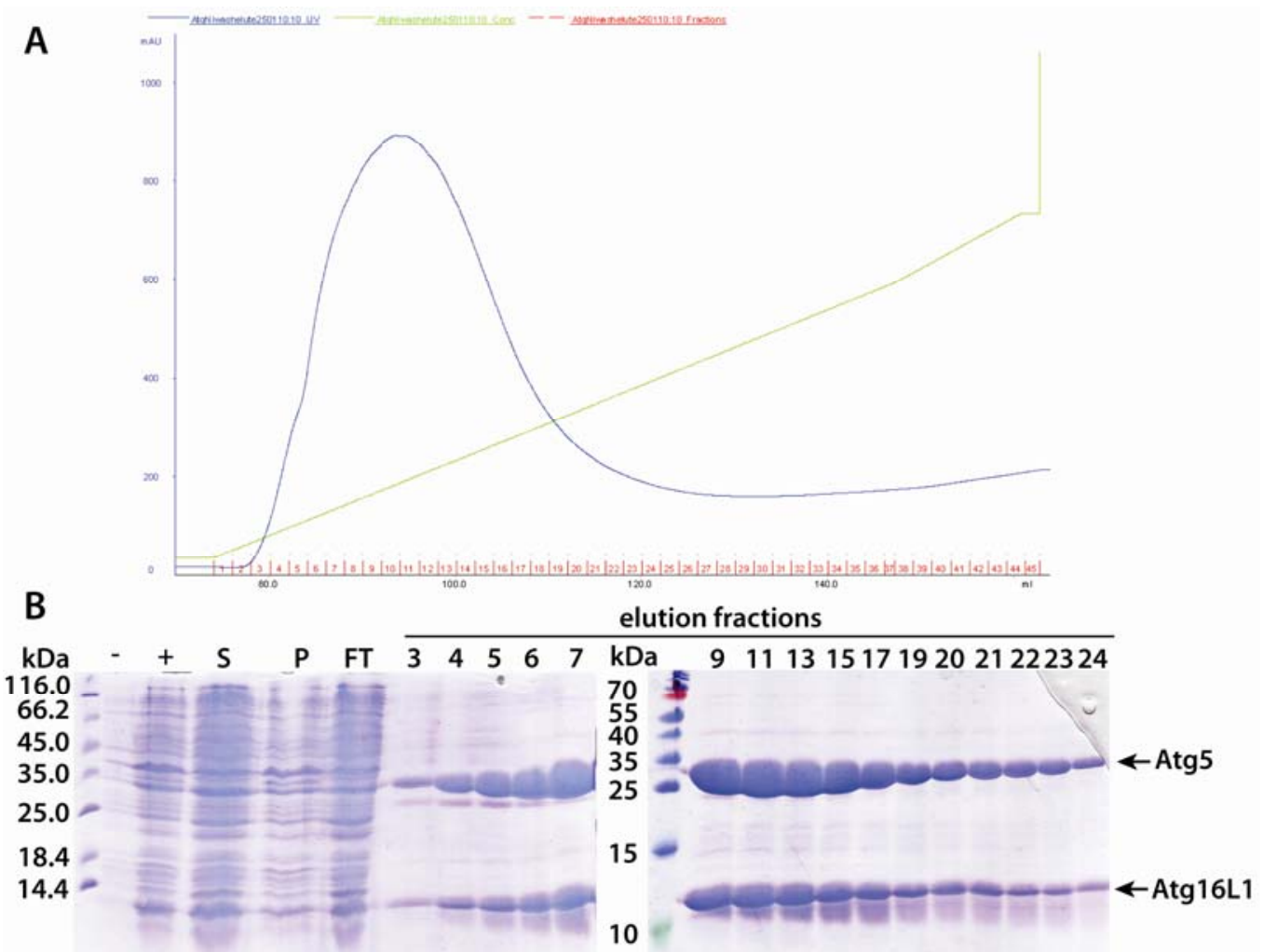

Figure 3.4: HisTrap FF purification of Atg5 Atg16L1(1-70). (A) Chromatogram with two connected 1 $\mathrm{mL}$ HisTrap FF columns. (B) 15\% SDS-PAGE gels of the expression analysis and elution fractions. - = uninduced, $+=$ induced, $S=$ supernatant, $\mathrm{P}=$ pellet, $F T=$ flow-through.

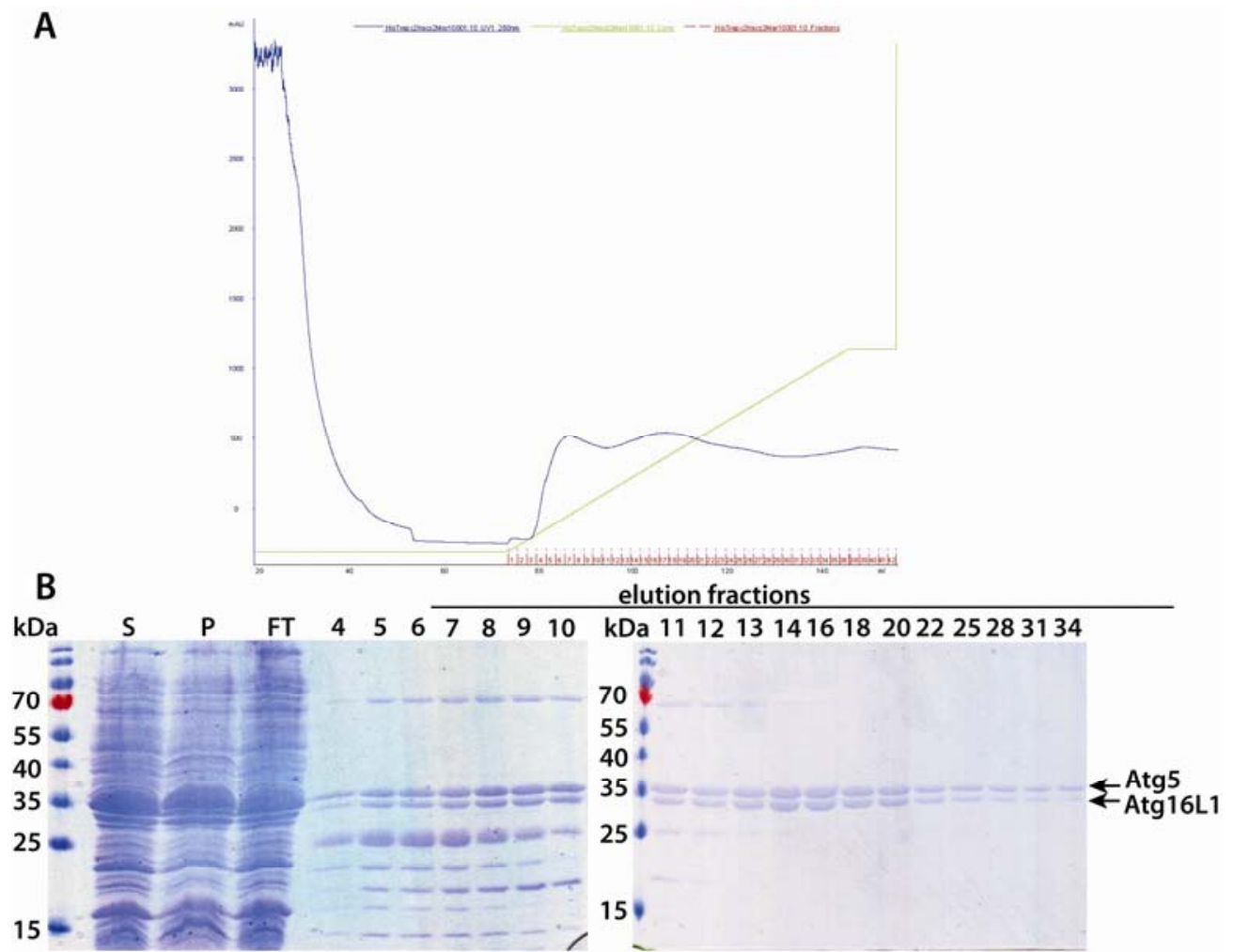

Figure 3.5: HisTrap FF purification of Atg5 Atg16L1(1-265). (A) Chromatogram with two connected 1 $\mathrm{mL}$ HisTrap FF columns. (B) 12\% SDS-PAGE gels of the expression analysis and elution fractions. $\mathrm{S}=$ supernatant, $\mathrm{P}=$ pellet, $\mathrm{FT}=$ flow-through, blue line $=\mathrm{UV}_{280}$ curve, green line $=\%$ elution buffer. 
The first 23 residues of Atg16L1 are important for association with Atg5. In their absence, dissociation of Atg16L1 from Atg5 is observed as evidenced by the reduction in co-elution from the gel filtration column (Fig. 3.7 panel B). In the absence of the first 52 residues of Atg16L1, binding to Atg5 was entirely abolished (Fig. 3.7 panel A).

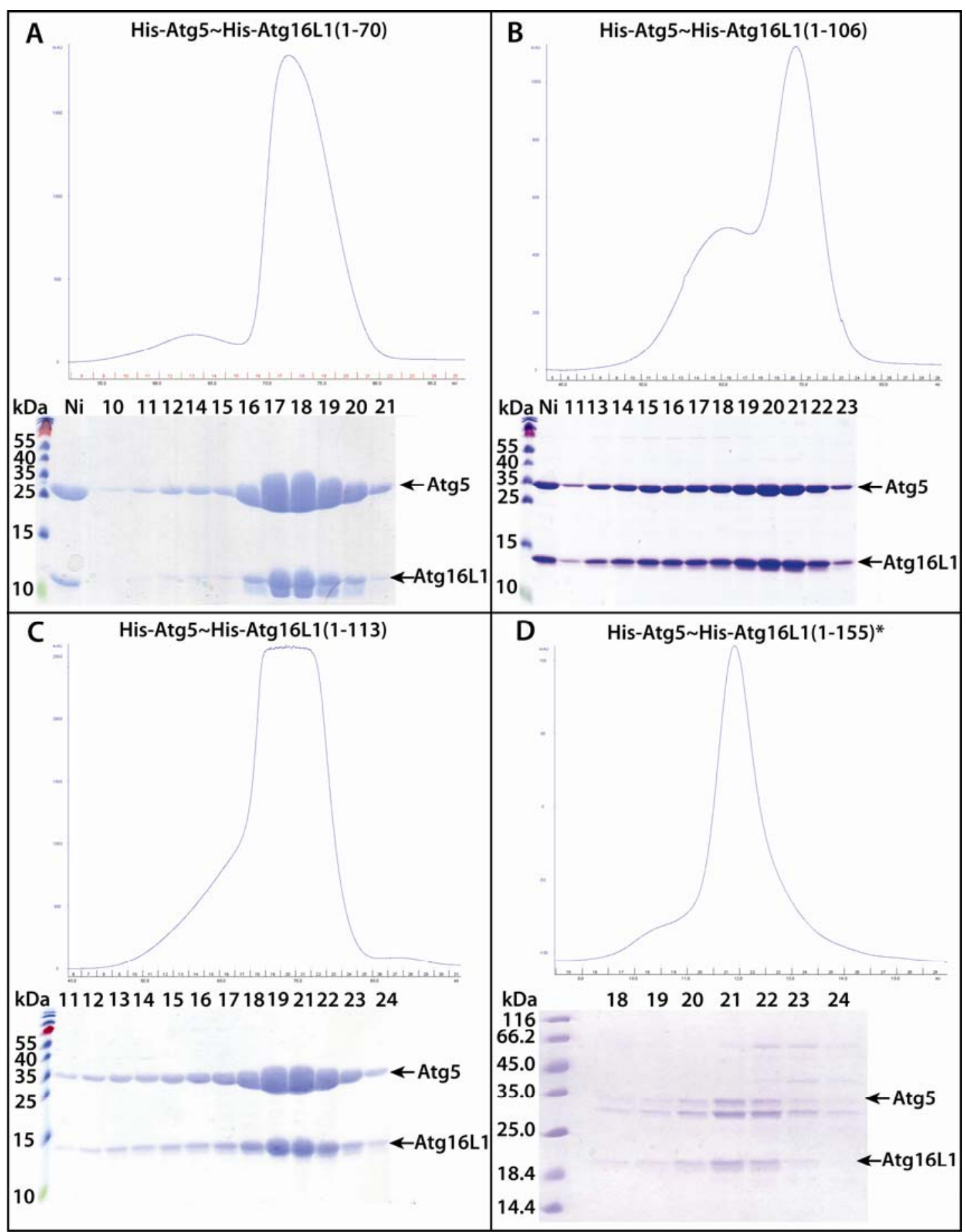

Figure 3.6: Gel filtration purification of Atg5 Atg16L1 complexes. Chromatograms and 15\% SDSPAGE gels of (A) Atg5 Atg16L1(1-70), (B) Atg5 Atg16L1(1-106), (C) His-Atg5 His-Atg16L1(1-113), and (D) Atg5 Atg16L1(1-155). * ${ }^{*}$ gel filtration using a Superdex 200 10/300 GL column instead of a HiLoad 16/60 Superdex 200 column. 
Purification of Atg16L1(53-168) allowed the oligomerization behaviour of Atg16L1 alone to be studied (further purification preps of Atg16L1(53-168) were performed by Dr. Karin Kühnel). The Atg5 Atg16L1(1-265), Atg5 Atg16L1(1-231), and Atg5 Atg16(1-168) complexes were purified with a final yield of $\sim 1.5-2 \mathrm{mg}$ per liter medium. The Atg5 Atg16L1(1-113), Atg5 Atg16L1(1-106), and Atg5 Atg16L1(1-70) complexes were purified with final yields of $\sim 15$ to $20 \mathrm{mg}$ per liter of culture. The Atg5 Atg16L1(24-168) complex was purified with a final yield of $\sim 0.5 \mathrm{mg}$ per liter of grown culture.

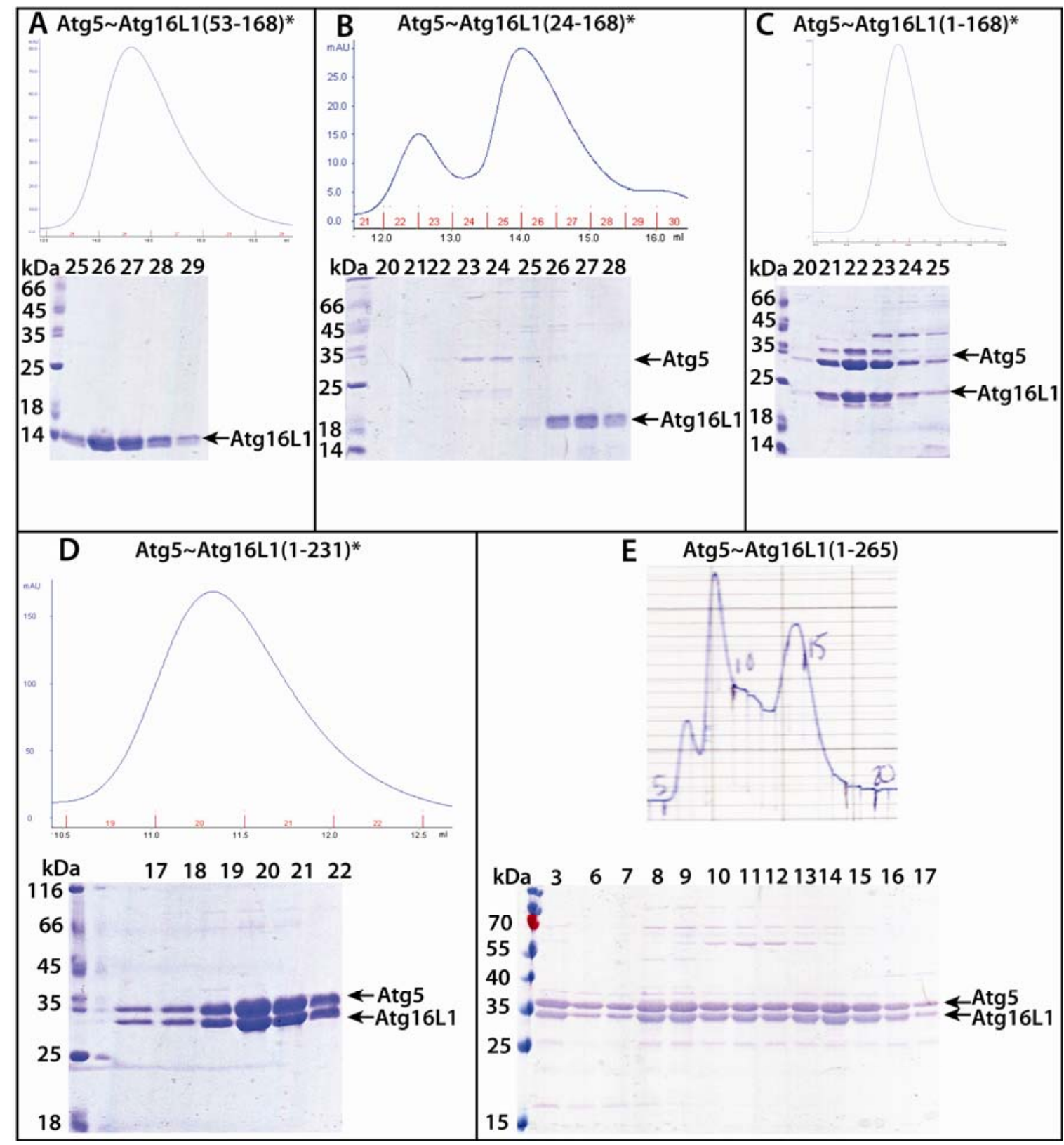

Figure 3.7: Gel filtration purification of Atg5 Atg16L1 complexes. Chromatograms and 15\% SDSPAGE gels of (A) Atg5 Atg16L1(53-168), (B) Atg5 Atg16L1(24-168), (C) Atg5 Atg16L1(1-168), (D) HisAtg5 His-Atg16L1(1-231), and (E) 12\% SDS-PAGE gel of His-Atg5 His-Atg16L1(1-265). * gel filtration with a Superdex 200 10/300 GL instead of the Superdex 200 16/60. 
Efforts were made to obtain an Atg5 Atg16L1 complex comprising both fulllength versions of the proteins; however Atg16L1(1-623) was found primarily in the insoluble pellet fraction (Figure 3.8). The yield after first-step IMAC purification was only $2 \mathrm{mg}$ per liter culture.

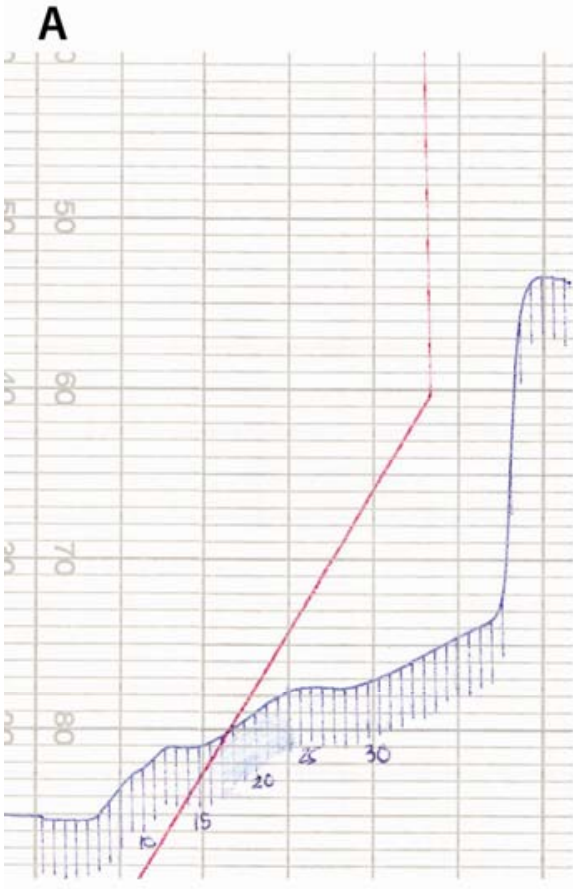

Figure 3.8: IMAC of full-length Atg5 Atg16L1(1-623). (A) Chromatogram of Ni-sepharose column purification of His-Atg5 His-Atg16L1(1-623). (B) 12\% SDS-PAGE gel of expression analysis showing most of the protein in the insoluble pellet fraction and the remaining soluble protein in the elution fractions. - = uninduced, $+=$ induced, $\mathrm{S}=$ supernatant, $\mathrm{P}=$ pellet, $\mathrm{FT}=$ flow-through, blue line $=\mathrm{UV}_{280}$ curve, red line $=\%$ elution buffer.

The insolubility of full-length Atg16L1 might have been due to the C-terminal WD40 repeat domain which is lacking in yeast Atg16. Another possibility is that a posttranslational modification is necessary to stabilize the protein and since the protein is overexpressed in E. coli, these post-translational modifications do not occur.

Insolubility can sometimes be overcome by adding a GST tag (Waugh 2005). Therefore the C-terminal WD40 repeat domain was cloned as a fusion protein with GST and purified with a glutathione sepharose column. The recombinant protein was mainly insoluble in the pellet fraction; however the remaining soluble protein was highly pure. It was further purified by size exclusion chromatography (Figure 3.9) and eluted in the void volume due to aggregation. Its final yield was approximately $3 \mathrm{mg}$ per liter grown culture. 

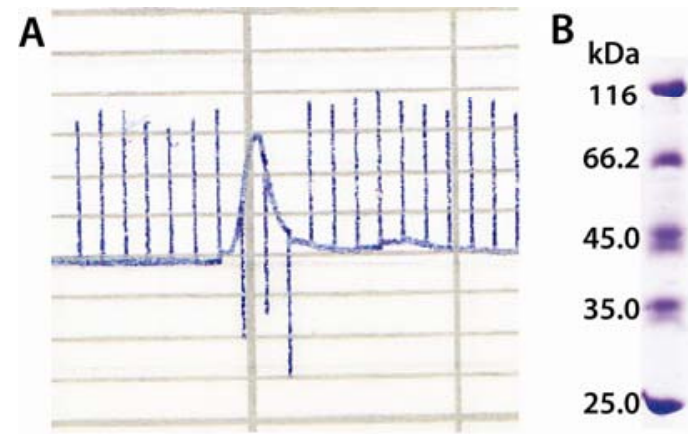

$\begin{array}{llllllll}6 & 7 & 8 & 9 & 10 & 11 & 12 & 13\end{array}$

Figure 3.9: Gel filtration purification of GST-Atg16L1(328-623). (A) Chromatogram and (B) 12\% SDSPAGE gel of GST-Atg16L1(328-623) after purification from a HiLoad 16/60 Superdex 75 column.

\subsubsection{Oligomerization state determination of $\operatorname{Atg} 5 \sim \operatorname{Atg} 16 \mathrm{~L} 1$ complexes}

During autophagosome formation, multimerization of the Atg12-Atg5 Atg16L1 complex via homo-oligomerization of the coiled coil domain of Atg16L1 is essential for isolation membrane expansion (Mizushima 1998, 2003). Further characterization of the multimerization state of the mammalian Atg5 Atg16L1 complex was carried out using analytical gel filtration and multiple angle laser light scattering (MALLS) experiments. The constructs used are summarized in Figure 3.10.
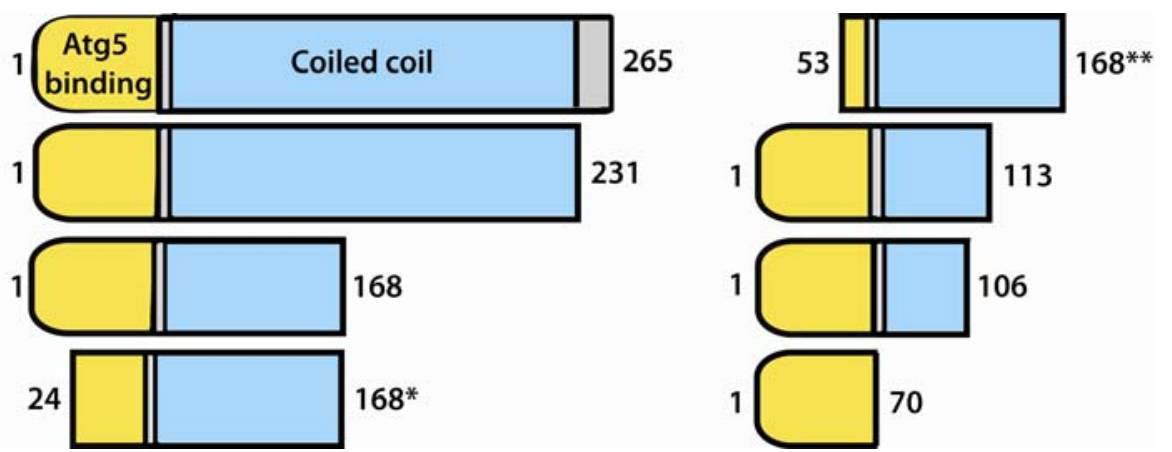

Figure 3.10: Constructs of mammalian Atg16L1 co-purified with Atg 5 that were used to determine the oligomerization state of the complex. ${ }^{*}$ was only used in the MALLS experiments, ${ }^{* *}$ was purified on its own without Atg5.

Analytical size exclusion chromatography was first used to confirm oligomerization of the Atg5 Atg16L1 complex. The Superdex 200 10/300 GL column was calibrated with proteins of known molecular weight to generate a standard curve (Figure 3.11 panel B). As seen in Figure 3.11 panel A, the Atg5 Atg16L1 complexes and Atg16L1 alone eluted at volumes corresponding to species larger than the monomeric proteins. Elution volumes were used to calculate the molecular weights of the proteins using the standard curve (Table 3.1). 


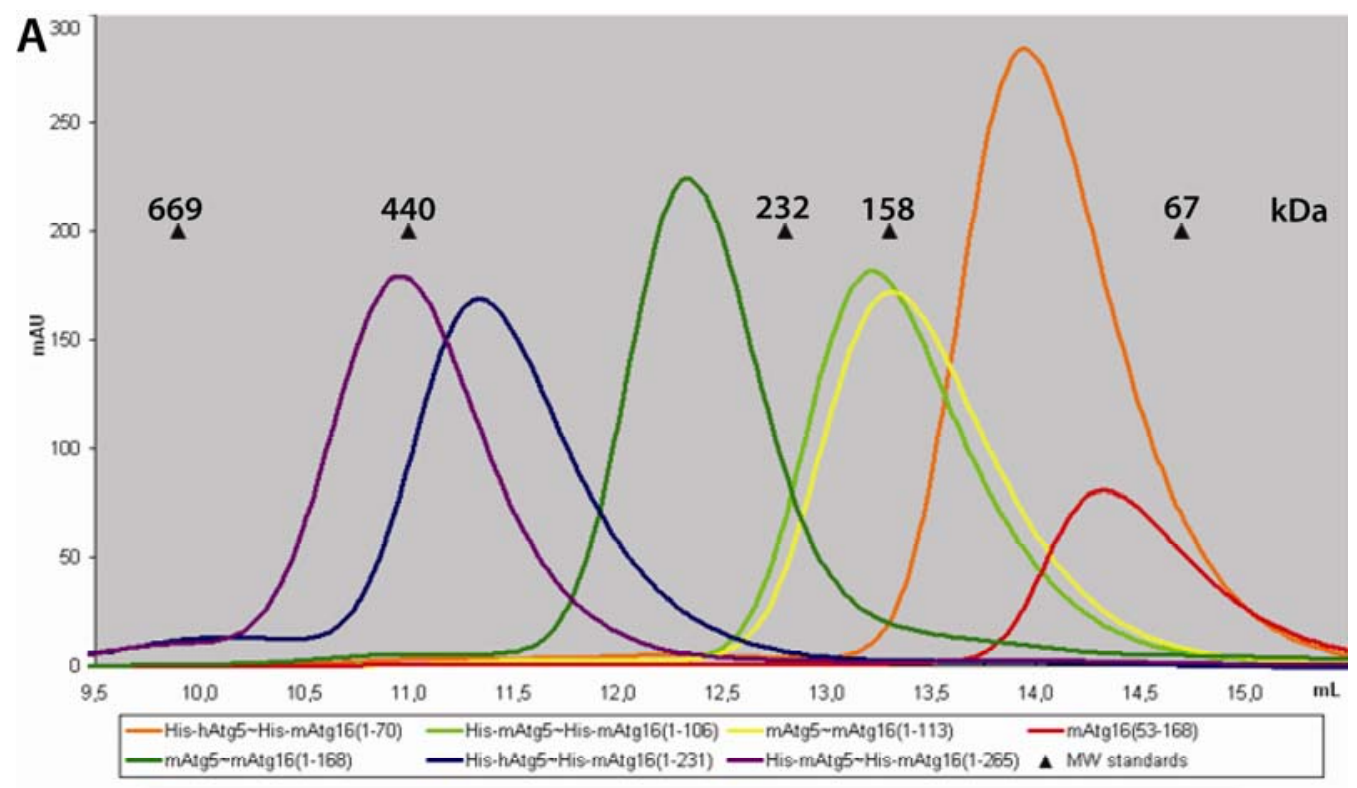

B

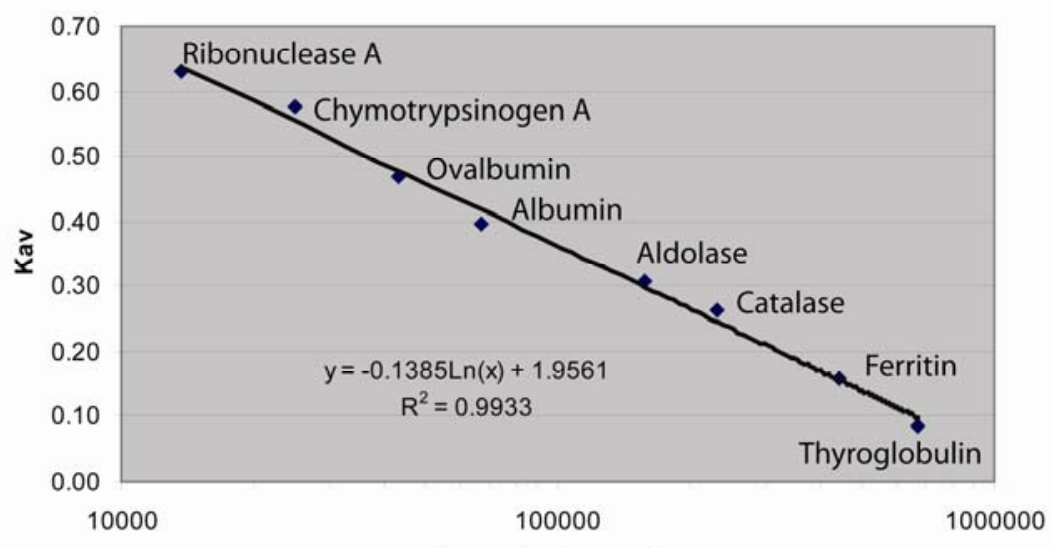

log molecular weight

Figure 3.11: Analytical gel filtration of the Atg5 Atg16L1 complexes. (A) Overlaid chromatograms of multimerized Atg5 Atg16L1 complexes or Atg16L1 on its own. (B) Calibration curve generated using molecular weight protein standards (Pharmacia) and a Superdex 200 10/300 GL column. $\mathrm{K}_{\mathrm{av}}=\left(\mathrm{V}_{\mathrm{e}}-\mathrm{V}_{\mathrm{o}}\right) /\left(\mathrm{V}_{\mathrm{t}}-\mathrm{V}_{\mathrm{o}}\right)$ where $\mathrm{K}_{\mathrm{av}}=$ gel phase distribution coefficient, $\mathrm{V}_{\mathrm{e}}=$ elution volume of the protein, $\mathrm{V}_{\mathrm{o}}=$ void volume of the column, $\mathrm{V}_{\mathrm{t}}=$ total column bed volume.

Table 3.1: Analytical gel filtration of Atg16L1(53-168) and Atg5 Atg16L1 complexes

\begin{tabular}{|c|c|c|c|c|c|}
\hline $\operatorname{Atg5(1-275)}$ & & & $\begin{array}{c}\text { multimerized } \\
\text { complex (kDa) }\end{array}$ & $\begin{array}{c}\text { monomeric } \\
\text { complex (kDa) }\end{array}$ & $\begin{array}{c}\text { ratio } \\
\text { oligomer: } \\
\text { monomer }\end{array}$ \\
\hline & mAtg16L & $(53-168)$ & \multirow{2}{*}{91.2} & 13.6 & 6.7 \\
\hline His-hAtg5 & $\begin{array}{r}\text { His } \\
\text { mAtg16L }\end{array}$ & $(1-70)$ & & 44.5 & 2.4 \\
\hline His-mAtg5 & $\begin{array}{r}\text { His } \\
\text { mAtg16L } \\
\end{array}$ & $(1-106)$ & 153.9 & 50.5 & 3 \\
\hline mAtg5 & mAtg16L & $(1-113)$ & 146.9 & 45.9 & 3.2 \\
\hline mAtg5 & mAtg16L1 & $(1-168)$ & 232.3 & 52.4 & 4.4 \\
\hline His-hAtg5 & mAtg16L1 & $(1-231)$ & 369.1 & 63.3 & 5,8 \\
\hline His-mAtg5 & mAtg16L & $(1-265)$ & 440.9 & 68.8 & 6.4 \\
\hline
\end{tabular}


While Atg16L1(53-168) and the Atg5 Atg16L1 complexes clearly multimerized, they did not display a consistency in their oligomerization stoichiometry. In fact, as the length of Atg16L1 in complex with Atg5 increased, so too did the oligomerization ratio of the complexes from a ratio of 2 for Atg5 Atg16L1(1-70) to 6 for the Atg5 Atg16L1(1265) complex (Table 3.1). However, the molecular weight calculated from the elution volume greatly depends on the shape of the molecule. For a non-globular protein, an apparent higher molecular weight would be observed. Here, the extended shape of the coiled coil domain made it appear as though the Atg5 Atg16L1 complexes had a higher molecular weight.

One way to offset the bias introduced by the non-globular shape of the coiled coil domain of Atg16L1 was to determine the molecular mass by MALLS (Figure 3.12).

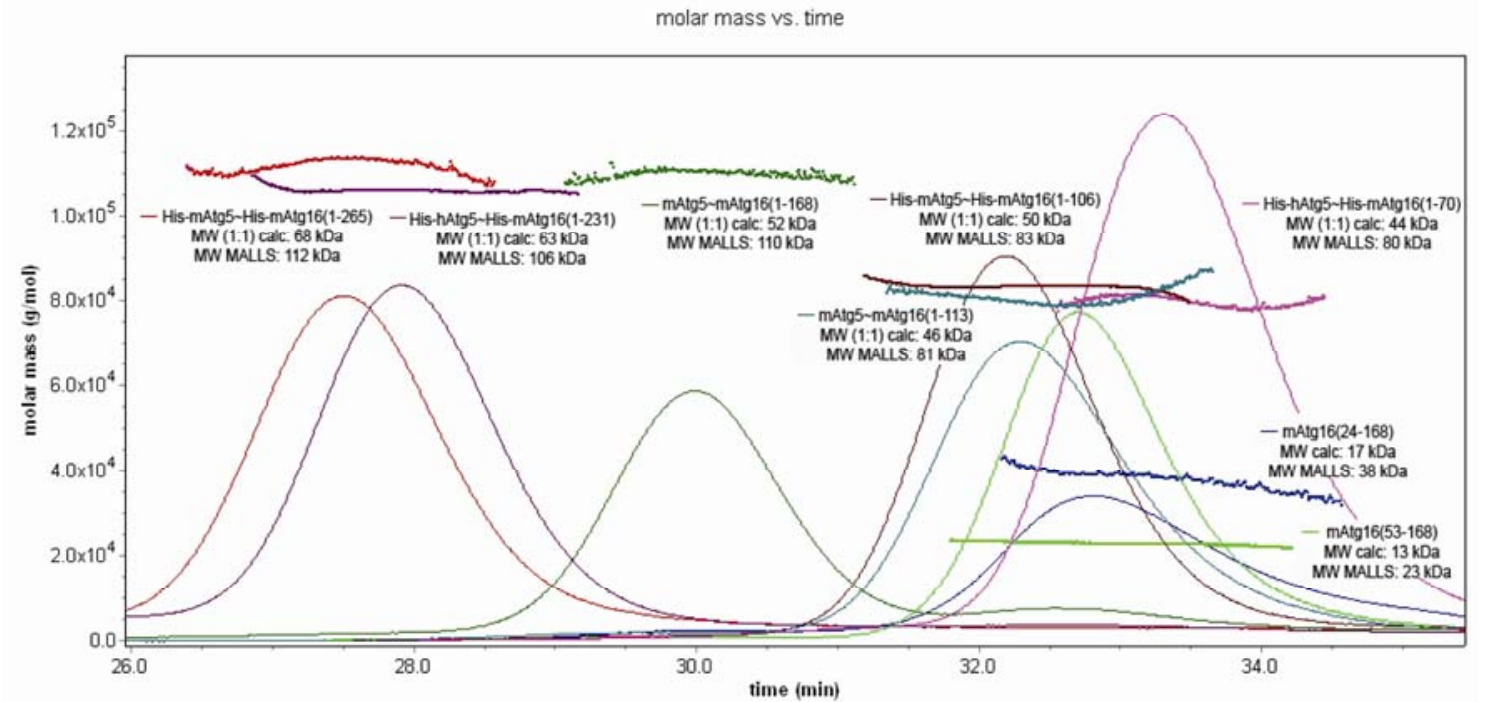

Figure 3.12: MALLS of Atg16L1(53-168) and Atg5 Atg16L1 complexes. The peaks correspond to their elution volumes. The horizontal line of the same color corresponds to the molar mass ( $y$-axis).

Table 3.2: MALLS of Atg16L1(53-168) and Atg5 Atg16L1 complexes

\begin{tabular}{|c|c|c|c|c|}
\hline $\operatorname{Atg5(1-275)}$ & $\operatorname{Atg} 16 L 1$ & $\begin{array}{c}\text { multimerized } \\
\text { complex (kDa) }\end{array}$ & $\begin{array}{c}\text { monomeric } \\
\text { complex (kDa) }\end{array}$ & $\begin{array}{c}\text { multimerized: } \\
\text { monomeric }\end{array}$ \\
\hline & mAtg16L1 (53-168) & 22.8 & 13.6 & 1.7 \\
\hline His-hAtg5 & His- mAtg16L1 (1-70) & 79.8 & 44.5 & 1.8 \\
\hline His-mAtg5 & His- mAtg16L1 (1-106) & 83.2 & 50.5 & 1.6 \\
\hline mAtg5 & mAtg16L1 (1-113) & 81.4 & 45.9 & 1.8 \\
\hline $\operatorname{mAtg} 5 \sim$ & mAtg16L1 (1-168) & 110 & 52.4 & 2.1 \\
\hline His-hAtg5 & mAtg16L1 (1-231) & 106 & 63.3 & 1.7 \\
\hline His-mAtg5 & mAtg16L1 (1-265) & 112 & 68.8 & 1.6 \\
\hline
\end{tabular}


Analysis of the determined molecular weights (Table 3.2) of Atg16L1(53-168) and the Atg5 Atg16L1 complexes shows that Atg16L1(53-168) is present as a dimer. Furthermore the Atg5 Atg16L1 complexes all form dimers consisting of two copies of Atg16L1 and Atg5 each. Of note, the Atg5 Atg16L1(1-70) complex which consisted of only the N-terminal Atg5-binding region of Atg16L1 was also present as a dimer. The Atg16L1 coiled coil domain is predicted by the program COILS (Lupas 1991) to begin at approximately residue 80. Dimerization of the minimal Atg5 Atg16L1 complex could be mediated by electrostatic interactions. If this is so, using high salt concentrations would inhibit dimer formation.

\subsubsection{Stability test and limited proteolysis experiments of Atg5 Atg16L1(1-231)}

Crystallization can take days, even months or longer, therefore the stability of the mammalian Atg5 Atg16L1(1-231) complex was tested. The complex was kept at room temperature and samples were taken at regular intervals. These samples were analyzed on an SDS-PAGE gel and showed that the complex remained intact over a period of nine days (Figure 3.13) and should be stable during crystallization trials.

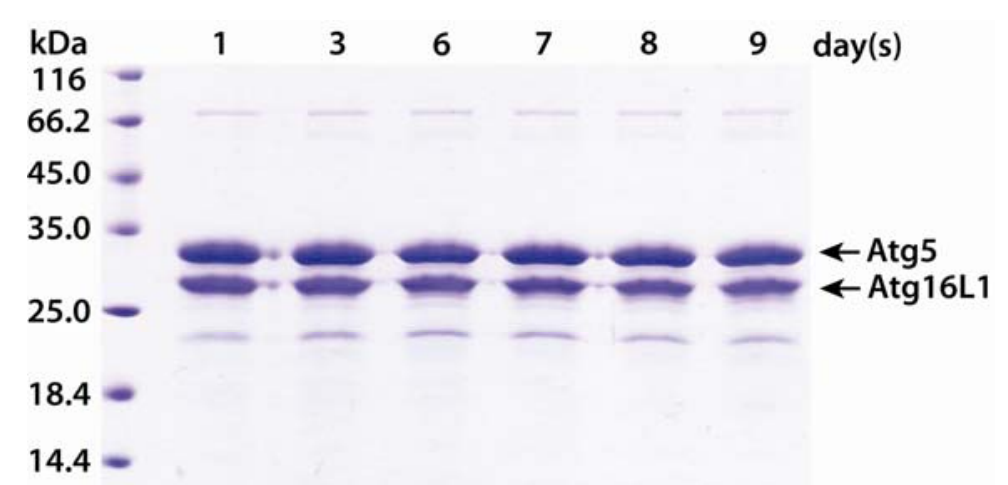

Figure 3.13: Stability test of the Atg5 Atg16L1(1-231) complex at room temperature. 15\% SDSPAGE gel showing the protein complex after incubation at RT over nine days with samples taken at the intervals indicated.

However, for crystallization it is often useful to identify shorter fragments of the protein of interest (Dale 2003). These fragments most likely are more compact and contain fewer disordered and flexible regions which might hinder crystallization. The goal was to identify stable fragments of Atg16L1 which interact with Atg5.

Limited proteolysis experiments of the Atg5 Atg16L1(1-231) complex using trypsin and chymotrypsin were performed. Digestions yielded several shorter, stable bands (Figure 3.14 panel A). To determine if the cleaveage products could still interact 
with Atg5, the digested samples were loaded onto a $1 \mathrm{~mL}$ HisTrap FF column. An $\sim 18$ $\mathrm{kDa}$ fragment co-eluted with Atg5 (Figure 3.14 panel B). A sample containing this fragment was blotted onto PVDF membrane for N-terminal sequencing (Seqlab, Göttingen). The identified residues were L-Q-A-E which corresponds to residues 56-59 of Atg16L1. According to the size of the fragment $(\sim 20 \mathrm{kDa})$, given that it began at amino acid residue 56, it most likely included the complete coiled coil domain up to residue 231 .

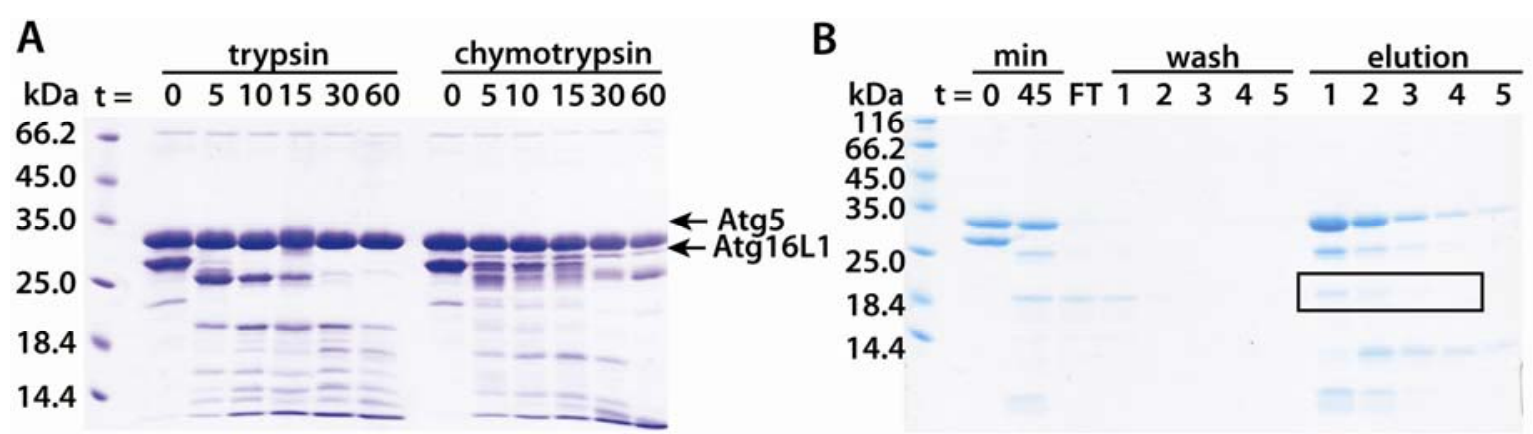

Figure 3.14: Limited proteolysis experiments of Atg5 Atg16L1(1-231). (A) 15\% SDS-PAGE gel of trypsin and chymotrypsin digestion time courses (in min). Proteases were added in a ratio of 1:200 (w/w) and incubated at RT for the time indicated. (B) 15\% SDS-PAGE gel of Atg5 Atg16L1(1-231) after 45 min digestion with trypsin. The reaction was stopped with $4 \mathrm{mM}$ Pefabloc and loaded onto a $1 \mathrm{~mL}$ HisTrap FF column. The box indicates the fragment sent for $\mathrm{N}$-terminal sequencing.

The identification of this stable Atg16L1 fragment led to the cloning of the Atg16L1(53-168) construct. Residue 168 was chosen as the ending residue because this comprised just over half of the coiled coil domain and had the lowest predicted disorder compared to the remaining coiled coil domain according to the DisEMBL protein disorder predictor 1.5 (Linding 2003). However, despite the co-elution of the sequenced fragment with Atg5 (Figure 3.14), Atg16L1(53-168) was seen to completely dissociate from Atg5 during purification when both proteins were co-expressed (3.7 panel A). Atg16L1(53168) did not yield crystals but was used for oligomerization studies (see Chapter 3.1.2).

\subsubsection{Crystallization of mammalian Atg5 Atg16L1 complexes}

When this project began, the structure of full-length yeast Atg5 in complex with the N-terminal portion of yeast Atg16(1-57) had been recently published (Matsushita 2007). At that time it had been determined that the yeast Atg12-Atg5 Atg16 complex formed a tetrameric $\sim 350 \mathrm{kDa}$ complex (Kuma 2002). In contrast, the molecular weight of the murine Atg12-Atg5 Atg16L1 complex was observed to be $\sim 800 \mathrm{kDa}$, which would correspond to 8 copies of each protein. The oligomerization of Atg16 is a requirement for 
formation of the autophagosome (Kuma 2002). With the aim of elucidating how Atg16L1 mediates multimerization of the Atg12-Atg5 Atg16L1 complex, I set out to crystallize an oligomerized mammalian Atg5 Atg16L1 complex. The Atg5 Atg16L1 complexes used for crystallization trials are summarized in Figure 3.15.

I also tried to crystallize the murine Atg5 Atg16L1(1-70) complex in order to compare it with the yeast Atg5 Atg16(1-57) structure. Crystallization screening was done with the Qiagen screens using 96-well plates with the sitting drop method at $20{ }^{\circ} \mathrm{C}$.
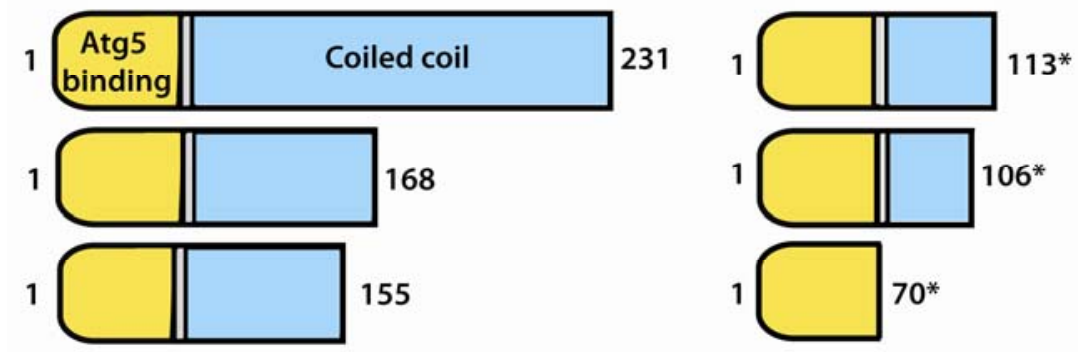

Figure 3.15: Constructs of murine Atg16L1 co-purified with human or murine Atg5 used for crystallization screening. The Atg16 1 1 (1-231) and (1-70) constructs were co-purified with human Atg5 which did not contain a His-tag cleavage site. The other Atg16L1 constructs were co-purified with murine Atg5, the His-tag of which could be cleaved off. * constructs produced crystals.

\section{The mammalian Atg5 Atg16L1(1-70) complex}

Crystals of Atg5 Atg16(1-70) grew in approximately four days in conditions of the commercial screens (Figure 3.16). These initial hits (Table 3.3) were then used to set up optimization screens in 24-well Linbro plates using the hanging drop vapor diffusion method. The $\mathrm{pH}$ and concentration of precipitant were varied to find the best conditions for the growth of single, large crystals. In addition, streak seeding was also performed. The optimized conditions are summarized in Table 3.4. Growth of crystals in the 24-well plates typically took up to 2 weeks.

Table 3.3: Initial crystallization conditions for the Atg5 Atg16L1(1-70) complex

\begin{tabular}{|c|c|c|c|c|c|c|}
\hline & Buffer & $\begin{array}{c}\text { Precipitant } \\
(w / v)\end{array}$ & Salt & $\begin{array}{l}\text { [Protein] } \\
(\mathrm{mg} / \mathrm{mL})\end{array}$ & $\begin{array}{l}\text { Drop } \\
\text { setup }\end{array}$ & Temp. \\
\hline 1 & $0.1 \mathrm{M}$ HEPES $\mathrm{pH} 7.0$ & $15 \%$ PEG 4000 & $0.1 \mathrm{M} \mathrm{MgCl}_{2}$ & 30 & $100: 100 \mathrm{~nL}$ & $20^{\circ} \mathrm{C}$ \\
\hline 2 & $0.1 \mathrm{M}$ HEPES $\mathrm{pH} 7.5$ & $15 \%$ PEG 6000 & $0.1 \mathrm{M} \mathrm{KCl}$ & 30 & $100: 100 \mathrm{~nL}$ & $20^{\circ} \mathrm{C}$ \\
\hline 3 & -------------------------- & 20\% PEG 3350 & $\begin{array}{l}0.2 \mathrm{M} \text { magnesium } \\
\text { formate }\end{array}$ & 30 & 100:100 nL & $20^{\circ} \mathrm{C}$ \\
\hline 4 & $\begin{array}{c}0.24 \mathrm{M} \text { sodium malonate } \mathrm{pH} \\
7\end{array}$ & 20\% PEG 3350 & 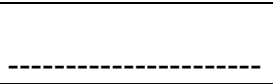 & 30 & $100: 100 \mathrm{~nL}$ & $20^{\circ} \mathrm{C}$ \\
\hline 5 & $0.1 \mathrm{M}$ succinic acid $\mathrm{pH} 7$ & 15\% PEG 3350 & ------------------------ & 30 & $100: 100 \mathrm{~nL}$ & $20^{\circ} \mathrm{C}$ \\
\hline 6 & 0.15 M DL-malic acid pH 7 & 20\% PEG 3350 & ------------------------ & 30 & $100: 100 \mathrm{~nL}$ & $20^{\circ} \mathrm{C}$ \\
\hline 7 & ----------------------------- & $20 \%$ PEG 3350 & $0.1 \mathrm{M} \mathrm{MgCl}_{2}$ & 30 & $100: 100 \mathrm{~nL}$ & $20^{\circ} \mathrm{C}$ \\
\hline
\end{tabular}




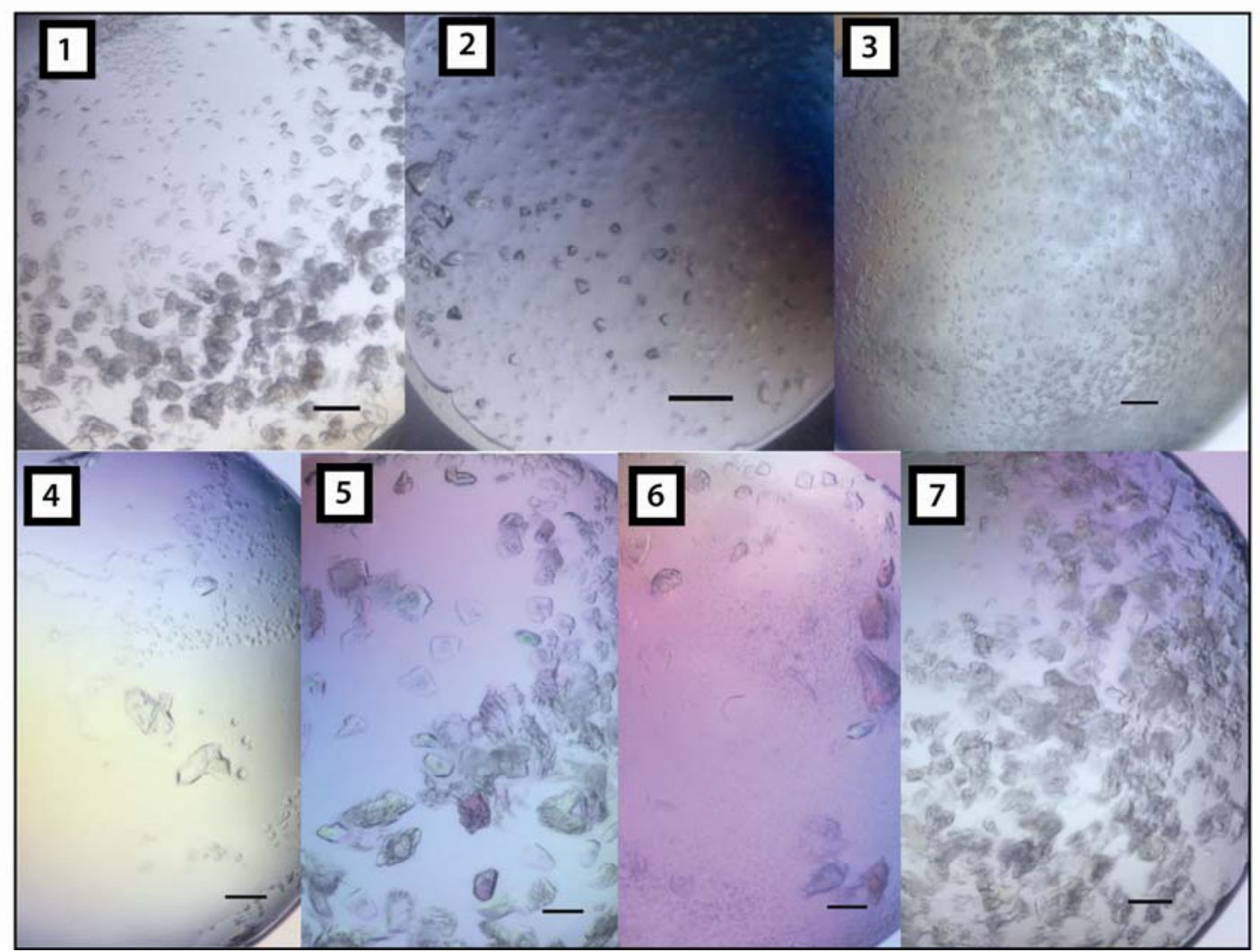

Figure 3.16: Crystals of the minimal mammalian Atg5 Atg16L1(1-70) complex. Numbers correspond to the crystallization conditions given in Table 3.3. Scale bar $=100 \mu \mathrm{m}$.

Table 3.4: Optimized crystallization conditions for the Atg5 Atg16L1(1-70) complex

\begin{tabular}{|c|l|c|c|c|c|}
\hline & \multicolumn{1}{c|}{ Buffer } & Precipitant (w/v) & [Protein] (mg/mL) & Drop setup & Temp. \\
\hline $\mathbf{8}$ & 0.24 M sodium malonate pH 7 & 20\% PEG 3350 & 30,20 & $1: 1 \mu \mathrm{L}$ & $20^{\circ} \mathrm{C}$ \\
\hline $\mathbf{9 *}^{*}$ & 0.1 M succinic acid pH 7 & 16\% PEG 3350 & 20 & $1: 1 \mu \mathrm{L}$ & $20^{\circ} \mathrm{C}$ \\
\hline $\mathbf{1 0 *}$ & 0.24 M sodium malonate pH 7 & 18\% PEG 3350 & 25 & $1: 1 \mu \mathrm{L}$ & $20^{\circ} \mathrm{C}$ \\
\hline $\mathbf{1 1}^{*}$ & 0.24 M sodium malonate pH 7 & 20\% PEG 3350 & 25 & $1: 1 \mu \mathrm{L}$ & $20^{\circ} \mathrm{C}$ \\
\hline $\mathbf{1 2}^{*}$ & 0.1 M succinic acid pH 7 & 16\% PEG 3350 & 10 & $1: 1 \mu \mathrm{L}$ & $20^{\circ} \mathrm{C}$ \\
\hline $\mathbf{1 3}^{*}$ & 0.1 M succinic acid pH 7 & 18\% PEG 3350 & 10 & $2: 1 \mu \mathrm{L}$ & $20^{\circ} \mathrm{C}$ \\
\hline $\begin{array}{l}\text { * Seeded } \\
\text { Buffer of protein=0.2 M NaCl, 30 mM HEPES pH 7.5 } \\
\text { Drop setup= protein:reservoir }\end{array}$ & & & & \\
\hline
\end{tabular}

Crystals were transferred into cryoprotectants using a 3-step serial dilution from mother liquor (reservoir solution) into 100\% cryoprotectant. Cryoprotectants were composed of mother liquor supplemented with $20-25 \%$ ethylene glycol or $10 \%$ xylitol/ $10 \%$ sucrose. After equilibration in cryoprotectant, the crystals were flash-cooled in liquid nitrogen. Crystals were sent to the Swiss Light Source (Switzerland) and their diffraction was tested at beamline PXII. However, these crystals diffracted very poorly and no data sets were collected. 


\section{The murine Atg5 Atg16L1(1-106) and Atg5 Atg16L1(1-113) complexes}

With the objective of determining the crystal structure of an oligomerized Atg5 Atg16L1 complex, various Atg5 Atg16L1 constructs differing in the lengths of the Atg16L1 coiled coil domain were used for crystallization trials at $20{ }^{\circ} \mathrm{C}$ (Figure 3.15). Crystals were obtained for two of the Atg5 Atg16L1 complexes. Crystals of the Atg5 Atg16L1(1-106) complex grew within two weeks. Crystals of the Atg5 Atg16L1(1-113) complex took between 2 to 6 weeks to grow (Figure 3.17). The conditions under which the crystals grew are summarized in Table 3.5. Crystals were then optimized by varying $\mathrm{pH}$ values, precipitant concentration, and streak seeding in 24-well Linbro plates using the hanging drop method.

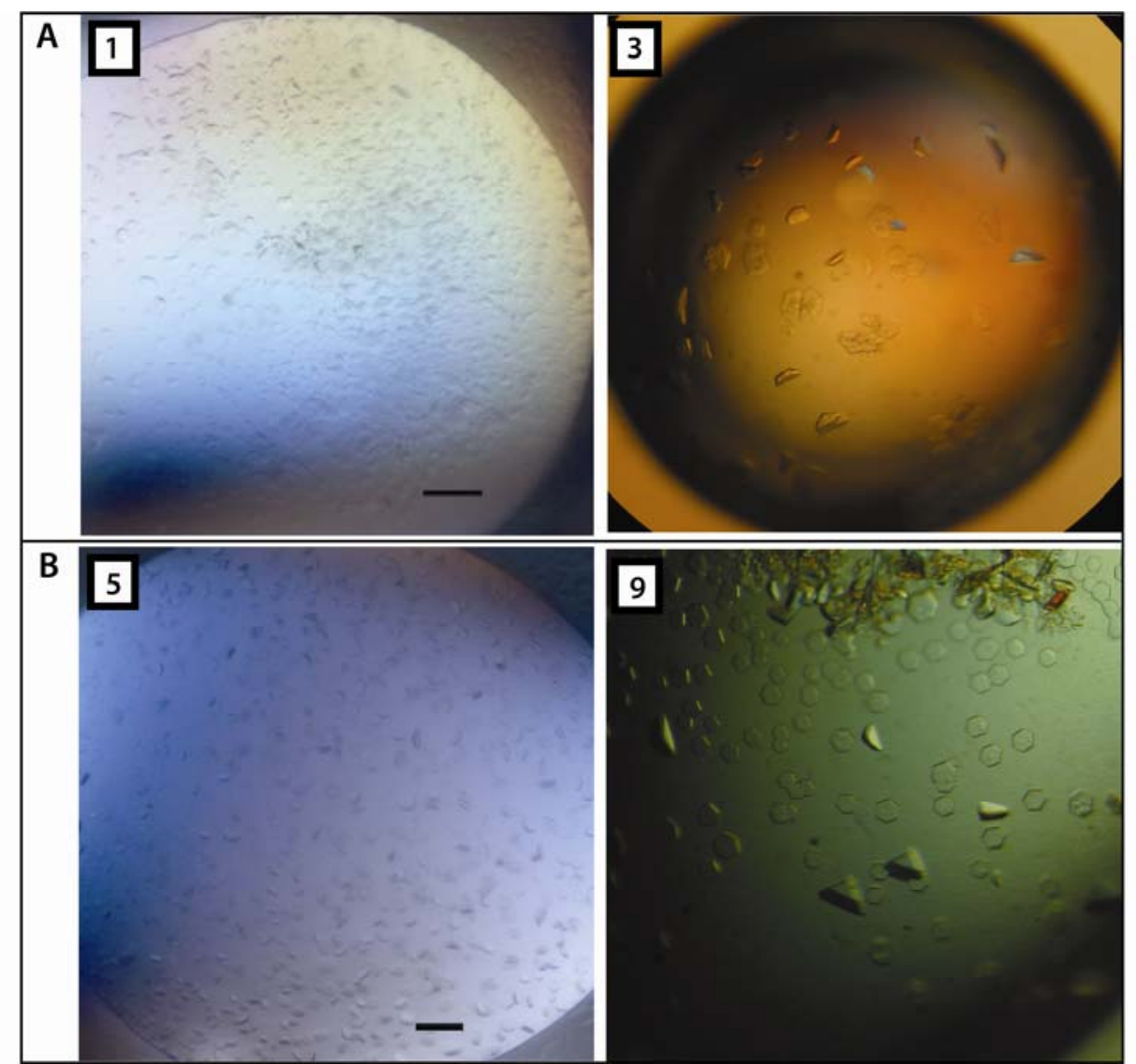

Figure 3.17: Crystals of the oligomerized mammalian Atg5 Atg16L1 complex. Crystals of the (A) Atg5 Atg16(1-106) and (B) Atg5 Atg16(1-113) complexes. Numbers correspond to the crystallization conditions listed in Table 3.5. Scale bar $=100 \mu \mathrm{m}$. 
Table 3.5: Crystallization conditions for Atg5 Atg16L1(1-106) and Atg5 Atg16L1(1-113)

\begin{tabular}{|c|c|c|c|c|c|c|}
\hline & $\begin{array}{c}\operatorname{Atg} 5 \sim \\
\operatorname{Atg} 16 \operatorname{L1}() \\
\end{array}$ & Buffer & Precipitant (w/v) & [Protein] & Drop setup & Temp. \\
\hline 1 & $(1-106)$ & 0.1 M HEPES $p H 7$ & $15 \%$ PEG 20000 & $37 \mathrm{mg} / \mathrm{mL}$ & $100: 100 \mathrm{~nL}$ & $20^{\circ} \mathrm{C}$ \\
\hline 2 & $(1-106)$ & $0.1 \mathrm{M}$ MES pH 6.5 & $9 \%$ PEG 35000 & $45 \mathrm{mg} / \mathrm{mL}$ & $1: 1 \mu \mathrm{L}$ & $20^{\circ} \mathrm{C}$ \\
\hline 3 & $(1-106)$ & $0.1 \mathrm{M}$ MES pH 6.3 & $6 \%$ PEG 35000 & $45 \mathrm{mg} / \mathrm{mL}$ & $1: 1 \mu \mathrm{L}$ & $20^{\circ} \mathrm{C}$ \\
\hline 4 & $(1-113)$ & $0.1 \mathrm{M}$ HEPES $\mathrm{pH} 7$ & $15 \%$ PEG 4000 & $37 \mathrm{mg} / \mathrm{mL}$ & $100: 100 \mathrm{~nL}$ & $20^{\circ} \mathrm{C}$ \\
\hline 5 & $(1-113)$ & $0.1 \mathrm{M}$ MES $\mathrm{pH} 6.5$ & $9 \%$ PEG 4000 & $30 \mathrm{mg} / \mathrm{mL}$ & $1: 1 \mu \mathrm{L}$ & $20^{\circ} \mathrm{C}$ \\
\hline 6 & $(1-113)$ & $0.1 \mathrm{M}$ HEPES pH 7 & $13 \%$ PEG 4000 & $30 \mathrm{mg} / \mathrm{mL}$ & $1: 1 \mu \mathrm{L}$ & $20^{\circ} \mathrm{C}$ \\
\hline 7 & $(1-113)$ & 0.1 M HEPES pH 7.5 & $17 \%$ PEG 4000 & $20 \mathrm{mg} / \mathrm{mL}$ & $2: 1 \mu \mathrm{L}$ & $20^{\circ} \mathrm{C}$ \\
\hline 8 & $(1-113)$ & 0.1 M MES pH 6.5 & $11 \%$ PEG 3350 & $30 \mathrm{mg} / \mathrm{mL}$ & $1: 1 \mu \mathrm{L}$ & $20^{\circ} \mathrm{C}$ \\
\hline 9 & $(1-113)$ & $0.1 \mathrm{M}$ HEPES $\mathrm{pH} 7$ & 9\% PEG 4000 & $45 \mathrm{mg} / \mathrm{mL}$ & $1: 1 \mu \mathrm{L}$ & $20{ }^{\circ} \mathrm{C}$ \\
\hline 10 & $(1-113)$ & $0.1 \mathrm{M}$ HEPES $\mathrm{pH} 7$ & $12 \%$ PEG 4000 & $45 \mathrm{mg} / \mathrm{mL}$ & $1: 1 \mu \mathrm{L}$ & $20^{\circ} \mathrm{C}$ \\
\hline $11^{*}$ & $(1-113)$ & 0.1 M HEPES pH 7.5 & $12 \%$ PEG 4000 & $50 \mathrm{mg} / \mathrm{mL}$ & $1: 2 \mu \mathrm{L}$ & $20^{\circ} \mathrm{C}$ \\
\hline
\end{tabular}

Crystals of murine Atg5 Atg16L1(1-106) were transferred stepwise into a cryoprotectant consisting of mother liquor supplemented with $25 \%$ ethylene. Crystals of murine Atg5 Atg16L1(1-113) were transferred to a cryoprotectant containing either $20 \%$ ethylene glycol or 20\% PEG 400 using a 3-step serial dilution. After equilibration in cryoprotectant, the crystals were flash-cooled in liquid nitrogen. Crystals were sent to the Swiss Light Source (Switzerland) and their diffraction was tested at beamline PXII. The cryoprotectants and quality of diffraction of the crystals measured are summarized in Table 3.6.

Table 3.6: Cryoprotection and diffraction resolution of Atg5 Atg16L1(1-106) and $\operatorname{Atg} 5 \sim \operatorname{Atg} 16 \mathrm{L1}(1-113)$ crystals

\begin{tabular}{|c|c|c|c|}
\hline & Construct & Cryoprotectant & Resolution of diffraction \\
\hline $\mathbf{3}$ & Atg5 Atg16(1-106) & $25 \%$ ethylene glycol & $5 \AA$ \\
\cline { 1 - 1 } $\mathbf{3}$ & Atg5 Atg16(1-106) & $25 \%$ ethylene glycol & $8 \AA$ \\
\cline { 1 - 1 } $\mathbf{3}$ & Atg5 Atg16(1-106) & $25 \%$ ethylene glycol & $9.5 \AA$ \\
\cline { 1 - 1 } $\mathbf{9}$ & Atg5 Atg16(1-113) & $20 \%$ ethylene glycol & $6 \AA$ \\
\cline { 1 - 1 } $\mathbf{9}$ & Atg5 Atg16(1-113) & 20\% PEG 400 & $7 \AA$ \\
\hline
\end{tabular}

Although the diffraction quality of the Atg5 Atg16L1(1-106) and Atg5 Atg16L1(1-113) crystals was not high enough to determine the structure, the $5 \AA$ resolution dataset collected for one of the Atg5 Atg16L1(1-106) crystals was sufficient to determine the unit cell parameters, its space group, and to calculate the self-rotation function. 


\subsubsection{X-ray data collection and processing of the Atg5 Atg16L1(1-106)}

Diffraction data of cryo-cooled crystals were collected at $100 \mathrm{~K}$ at beamline PXII (SLS, Switzerland). Data processing and scaling were done with the $X D S$ software package (Kabsch 2010a,b). Data collection statistics are summarized in Table 3.7.

The Matthews coefficient, $V_{\mathrm{M}}$, is defined as the crystal volume per unit of protein molecular weight (Matthews 1968, 1976). This value can be used to estimate the number of molecules in the asymmetric unit. Brian Matthews observed that the solvent occupies between $27 \%$ and $78 \%$ of the total crystal volume (Matthews 1968). The range of $V_{m}$ for protein crystals usually falls between $2.3 \AA^{3} / \mathrm{Da}$ and $2.7 \AA^{3} / \mathrm{Da}$ (Kantardjieff 2003). The Matthews coefficient program, as part of the CCP4 program suite, was used for calculations (Collaborative Computational Project Number 4 1994, Potterton 2003). Based on the molecular weight of the Atg5 Atg16L1(1-106) complex, the unit cell dimensions and space group (C2), it was estimated that there were most likely six to eight molecules of the complex per asymmetric unit (Table 3.8).

Table 3.7: Data collection statistics for $\operatorname{Atg5} \sim \operatorname{Atg} 16 \mathrm{L1}(1-106)$

\begin{tabular}{|c|c|}
\hline & \multirow{4}{*}{$\begin{array}{c}\text { Atg5 Ptg16L1(1-106) } \\
\text { C2 } \\
199.0,160.0,94.6\left(\beta=96.1^{\circ}\right) \\
\text { SLS PXII }\end{array}$} \\
\hline Space group & \\
\hline Unit cell dimensions $a, b, c,(\AA)$ & \\
\hline Beamline & \\
\hline Wavelength & $1.0 \AA$ \\
\hline Resolution of data $(\AA)$ (high-resolution bin) & $25-5.0(5-6.5)$ \\
\hline No. of observations/ no. unique reflections & $48824 / 12770$ \\
\hline completeness $(\%)$, total (high) & $99.7(99.9)$ \\
\hline I/oI, total (high) & $13.5(3.9)$ \\
\hline R-factor observed (\%), total (high) & $5.7(38.2)$ \\
\hline $\begin{array}{l}R \text {-factor }=\Sigma_{\mathrm{hkl}} \Sigma_{i}\left|I_{i}(\mathrm{hkl})-\langle I(\mathrm{hkl})\rangle\right| / \Sigma_{\mathrm{hk} l} \Sigma_{i}\left|I_{i}(\mathrm{hkl})\right| ; \text { fo } \\
\text { reflection, where }\langle I(\mathrm{hkl})\rangle=\text { average intensity of } \mathrm{t}\end{array}$ & eflections and $i$ observations of a given \\
\hline
\end{tabular}

Table 3.8. Matthews coefficient for Atg5 Atg16L1(1-106) crystals

\begin{tabular}{|c|c|c|}
\hline $\mathbf{N}$ & $\mathbf{V m}\left(\AA^{3} / \mathrm{Da}\right)$ & \% solvent \\
\hline 6 & 2.8 & 55.6 \\
\hline 7 & 2.4 & 48.1 \\
\hline 8 & 2.1 & 40.7 \\
\hline $\begin{array}{l}\mathrm{N}=\text { no. of molecules/asymmetric unit } \\
\text { Vm=Matthews coefficient }\end{array}$ \\
\hline
\end{tabular}


To characterize the content of the asymmetric unit and the nature of the noncrystallographic symmetry, a Patterson self-rotation function was calculated. The selfrotation function presents all crystallographic and non-crystallographic rotational symmetry axes as well as their orientations to one another. First, the measured structure factors are used to calculate two Patterson maps. The orientation of one is kept constant while the other is rotated in small increments and the two maps are overlaid and integrated. At certain angles which correspond to rotational symmetry elements found in the crystal, both maps will coincide resulting in a peak in the self-rotation function. The self-rotation function was calculated with MOLREP (Vagin 1997), which is part of the CCP4 program suite (Collaborative Computational Project Number 4 1994, Potterton 2003), using data between 25 and $6 \AA$ resolution and a Patterson radius of $30 \AA$. Results were visualized as stereographic projections. Peaks at chi $=180^{\circ}$ indicate the presence of 2-fold non-crystallographic symmetry axes. Six of the peaks present are due to 2-fold non-crystallographic symmetry and the peak originating from the 2-fold crystallographic symmetry axis along $b$ is marked with asterisks (Fig. 3.18). This indicates that there are three dimers of the Atg5 Atg16L1(1-106) complex in the asymmetric unit.

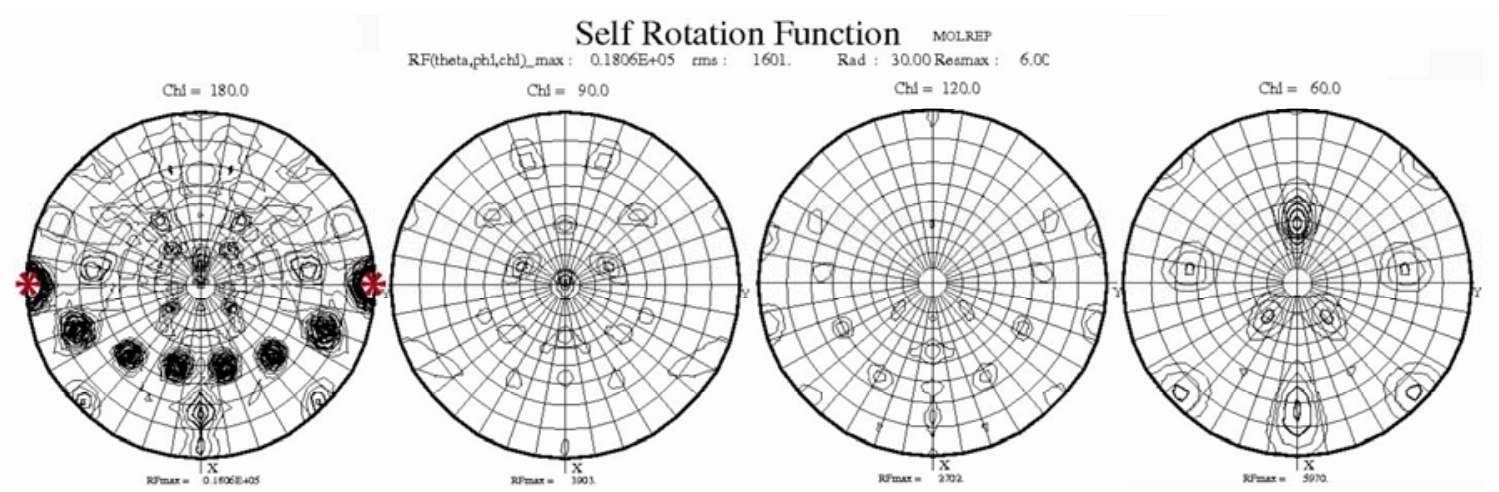

Figure 3.18: Stereographic projections of the self-rotation function of the Atg5 Atg16L1(1-106) complex. The stereographic projections are shown for different chi angles $\left(180^{\circ}, 90^{\circ}, 120^{\circ}\right.$, and $\left.60^{\circ}\right)$. The red asterisks represent the 2-fold crystallographic symmetry axis along b.

\subsubsection{Cleavage of $\operatorname{Atg} 16 \mathrm{~L} 1$ by the protease calpain}

Calpains are a family of cysteine proteases which are activated by calcium. Calpain-1 ( $\mu$-calpain) and calpain-2 (m-calpain) are the most common calpains and are ubiquitously expressed (reviewed in Goll 2003). In 2006, Yousefi et al. observed that Atg5 is cleaved by calpain to form a truncated $24 \mathrm{kDa}$ fragment comprising residues 1-193. They found that this fragment translocates to the mitochondria and associates with $\mathrm{Bcl}-\mathrm{x}_{\mathrm{L}}$, which triggers apoptosis. It was our aim to crystallize the complex of $\mathrm{Bcl}-\mathrm{x}_{\mathrm{L}}$ with the truncated form of Atg5. 
Adi Bar Shalom, a lab rotation student, tried to express Atg5(1-193) on its own as well as co-expression with Bcl- $\mathrm{X}_{\mathrm{L}}(1-44,85-196)$. Neither strategy yielded soluble protein. Therefore, I tried to generate the cleavage product of Atg5 by digesting the Atg5 Atg16L1(1-231) complex with calpain-1. However, upon addition of calpain-1 (Calbiochem) to the complex, Atg5 remained intact while Atg16L1(1-231) was almost entirely cleaved, resulting in the formation of several fragments (Figure 3.19 panel A). Upon addition of calpain-1, Atg16L1(1-231) was almost entirely digested within 7 hours at $4{ }^{\circ} \mathrm{C}$ and one hour at $\mathrm{RT}$, while Atg5 remained intact. In contrast, without the addition of calpain-1, the complex was stable at $4{ }^{\circ} \mathrm{C}$ and at RT for up to a day (Figure 3.19 panel B).

A

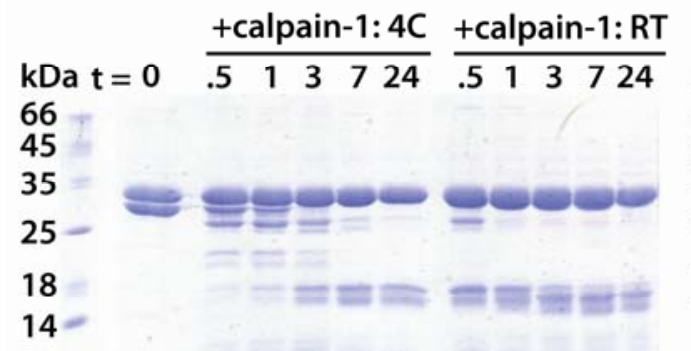

Figure 3.19: Calpain-1 cleavage of the Atg5 Atg16L1(1-231) complex. 15\% SDS-PAGE gels of time course comparing the stability of the Atg5 Atg16L1(1-231) complex (A) with and (B) without calpain-1 at $4{ }^{\circ} \mathrm{C}$ and RT. $\mathrm{t}=\mathrm{hr}$.

Calpain-2 (Calbiochem) was also tested. To investigate whether this cleavage was indeed due to calpains, several calpain inhibitors were added to the digestions: acetyl calpastatin(184-210) CS peptide (Anaspec) inhibits both of these calpains, whereas the peptides ALLN (Calbiochem) and ALLM (Sigma Aldrich) are specific for calpain-1 and calpain-2, respectively. As can be seen in Figure 3.20, both calpain-1 and calpain-2 cleaved Atg16L1(1-231) and this cleavage was blocked by the presence of ALLN, ALLM, or calpastatin. In contrast, Pefabloc SC (Roche), a serine protease inhibitor, did not inhibit cleavage. Together these results show that cleavage is indeed catalyzed by calpain.

To test if the N-terminal Atg5-binding domain of Atg16L1 is sufficient for calpain1 cleavage, the Atg5 Atg16L1(1-70) complex was also digested with calpain. Within 15 min of addition of calpain-1, Atg16L1(1-70) was cleaved into smaller fragments while Atg5 remained intact (Figure 3.21). In the absence of calpain-1 or in the presence of ALLN, Atg16L1(1-70) remained intact even after incubation for $90 \mathrm{~min}$ at $37^{\circ} \mathrm{C}$. 


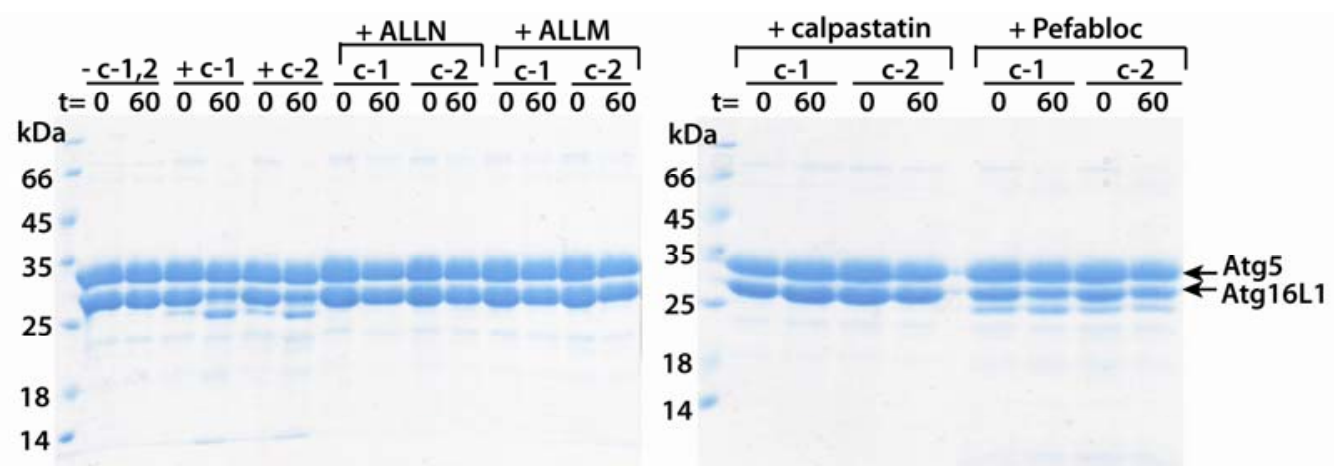

Figure 3.20: Cleavage of the Atg5 Atg16L1(1-231) complex by calpain-1 and calpain-2. 15\% SDSPAGE gels of the Atg5 Atg16L1(1-231) complex after incubation in the absence or presence of calpain-1 (c-1) and calpain-2 (c-2) (at a ratio of 1:50 w/w) and specific inhibitors ALLN, ALLM, and calpastatin at $37^{\circ} \mathrm{C}$ for $60 \mathrm{~min}$.

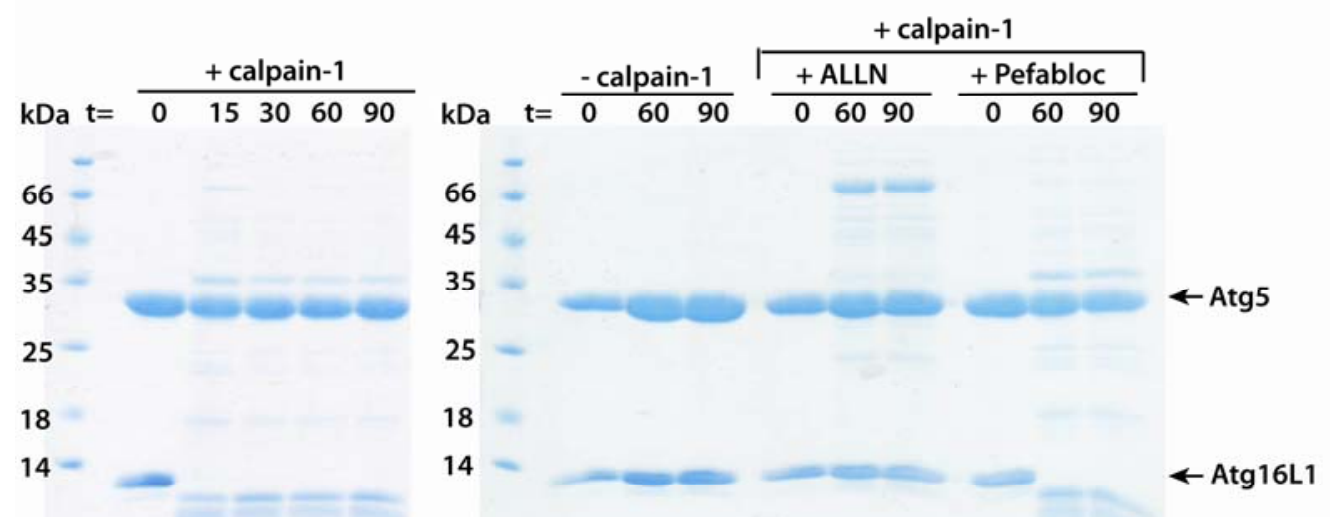

Figure 3.21: Calpain-1 also specifically cleaves Atg16L1(1-70). 15\% SDS-PAGE gels of time course of the Atg5 Atg16L1(1-70) complex after incubation at $37^{\circ} \mathrm{C}$ in the absence or presence (at a ratio of 1:50 $\mathrm{w} / \mathrm{w}$ ) of calpain-1 and its specific inhibitor ALLN. $\mathrm{t}=\mathrm{min}$.

To characterize where calpain cleaved Atg16L1, the Atg5 Atg16L1(1-231) complex was loaded onto a HisTrap FF column after calpain digestion for 60 min at $37^{\circ} \mathrm{C}$ (Figure 3.22). The Atg5-binding domain of Atg16L1 was intact since Atg5 co-eluted with an approximately $24 \mathrm{kDa}$ Atg16L1 cleavage product. The band of Atg16L1 shown in the box in Fig. 3.22 was sent for N-terminal sequencing and the first four residues were determined to be G-S-H-M. These amino acid residues correspond to the thrombin cleavage site in the Atg16L1 construct. This indicates that calpain cleaved near the C-terminus of Atg16L1(1-231). 


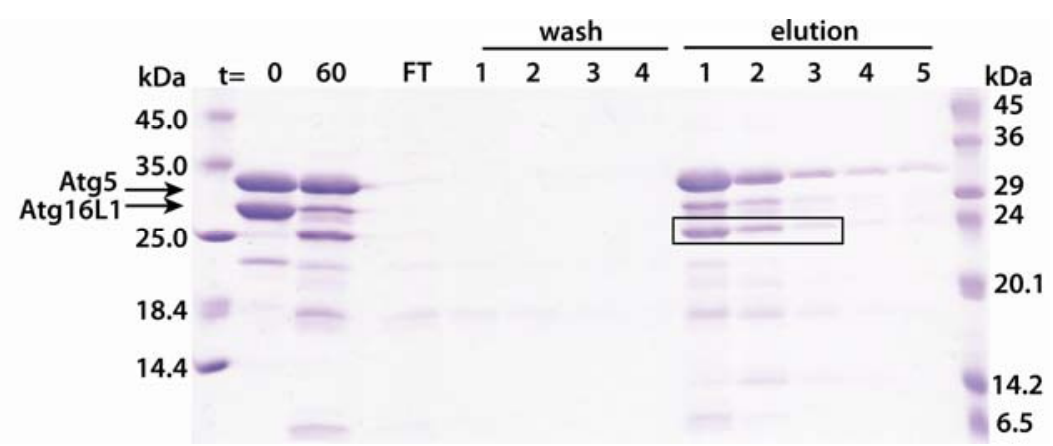

Figure 3.22: Cleaved Atg16L1 fragment co-elutes with Atg5. 15\% SDS-PAGE gel after incubation of calpain-1 (1:50 w/w) at $37^{\circ} \mathrm{C}$ for 60 min with Atg5 Atg16L1(1-231). The reaction was stopped with ALLN and loaded onto a HisTrap FF column. Several fragments of Atg16L1 co-eluted with Atg5. $t=\min$. Box indicates fragment of Atg16L1 sent for $\mathrm{N}$-terminal sequencing.

Atg16L1 might mask the calpain cleavage site of Atg5 when they form a complex. To test this hypothesis, calpain-1 was added to Atg5 that was purified on its own. As seen in Figure 3.23 panel A, full-length Atg5 on its own does not seem to diminish upon incubation with calpain-1 for 60 min whereas Atg16L1(1-231) is effectively cleaved by calpain-1 in the presence of Atg5, which remains intact. Of note, there was always a constant band of $\sim 24 \mathrm{kDa}$ present in the Atg5 sample, even without addition of calpain. The digestion was repeated again, and here the incubation time was doubled. It can be observed on a Western blot where an Atg5 antibody (rabbit anti-Apg5L polyclonal antibody, Proteintech Group Inc) was used for detection that a $\sim 24 \mathrm{kDa}$ band was present even in the absence of calpain and also when the calpain inhibitor ALLN was present (Figure 3.23 panel B). In addition, no band corresponding to an Atg5 cleavage product can be observed for the Atg5 Atg16L1(1-231) complex which was digested by calpain for two hours at $37{ }^{\circ} \mathrm{C}$. These data demonstrate that Atg5, either on its own or in complex with Atg16L1, is not cleaved by calpain.

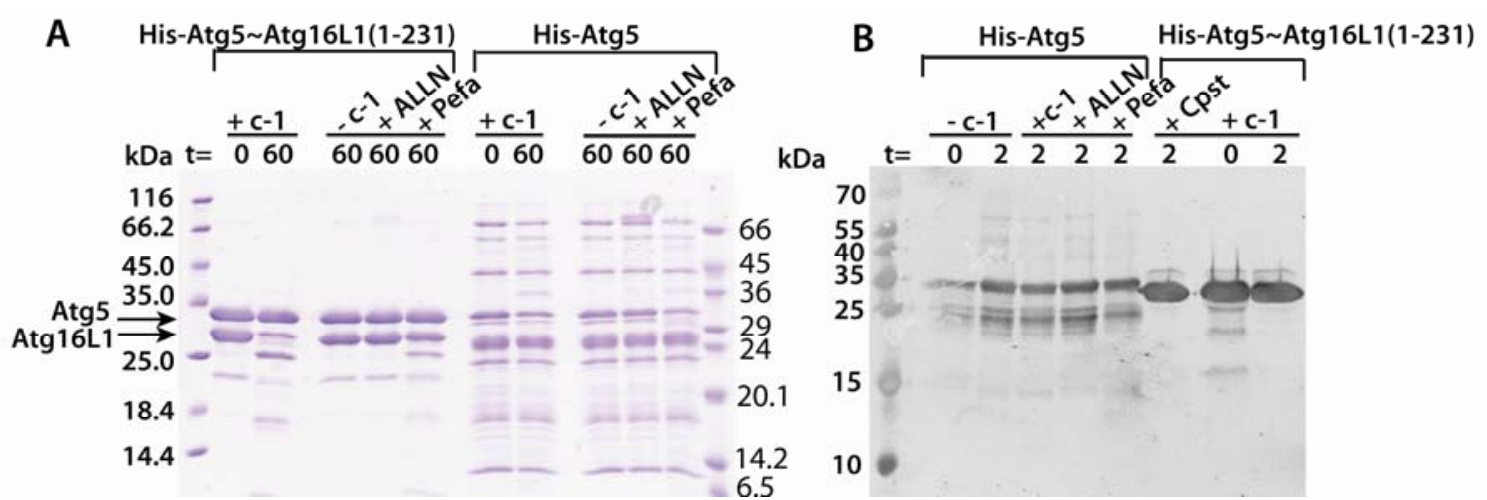

Figure 3.23: Calpain cleaves Atg16L1 but not Atg5. (A) 15\% SDS-PAGE gel of calpain-1 (at a ratio of 1:50 w/W) cleavage of Atg5 Atg16L1(1-231) or Atg5 for $60 \mathrm{~min}$ at $37^{\circ} \mathrm{C}$. (B) Western blot using polyclonal anti-Apg5L antibody of Atg5 and Atg5 Atg16L1(1-231) after incubation with calpain-1 (at a ratio of 1:50 $\mathrm{w} / \mathrm{w}$ ) for 120 minutes at $37^{\circ} \mathrm{C}$. A $24 \mathrm{kDa}$ fragment of Atg5 is observed even without addition of calpain $(\mathrm{c}-1)$ and is not enriched upon incubation with calpain. $\mathrm{t}=\mathrm{min} \mathrm{t}=\mathrm{hr}$, Pefa=pefabloc, cpst=calpastatin. 


\subsection{Characterization of mammalian Rab33B Atg16L1 complexes}

\subsubsection{Purification of Rab33BQ92L}

It was our goal to elucidate the exact mechanism of interaction between Rab33B and Atg16L1 by determining the structure of the Rab33B Atg16L1 complex. Atg16L1 interacts with Rab33B in a GTP-dependent manner, making it an effector of Rab33B (Itoh 2008). For this reason, all experiments were done with Rab33BQ92L. The glutamine to leucine mutation eliminates the intrinsic GTPase activity of Rab33B, trapping it in its GTP-bound state. Full-length murine Rab33B(1-229) was cloned as an N-terminal GSTfusion protein using the pGEX-4T-1 plasmid and was mutated at residue 92 from a glutamine to a leucine by two-step PCR. Despite being tagged with GST, Rab33B(1229)Q92L was mostly insoluble and accumulated in the pellet fraction (Figure 3.24). The little amount of protein that was eluted from the beads was partially degraded.

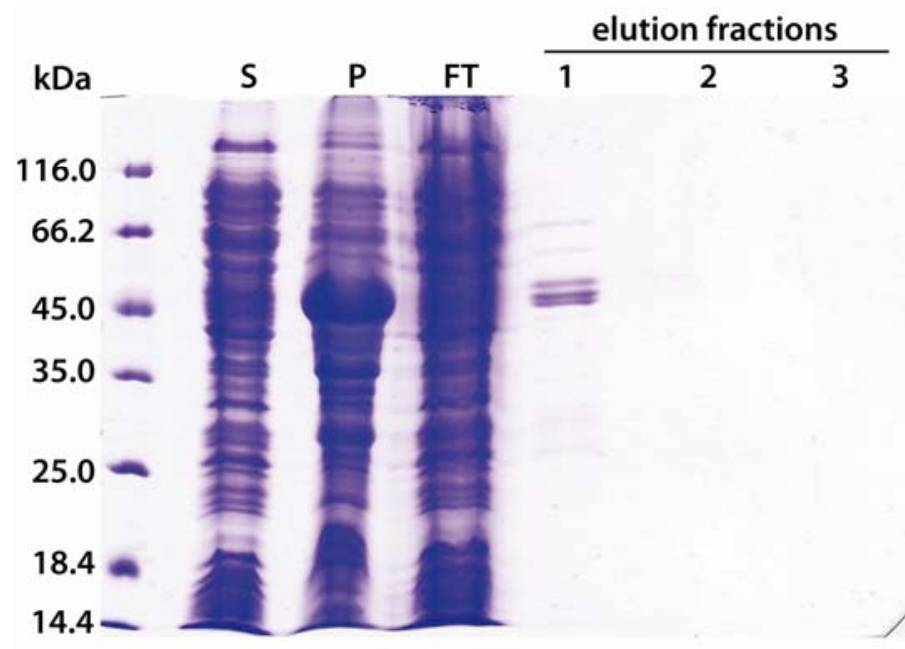

Figure 3.24: Purification of GST-Rab33B(1-229)Q92L. (A) 15\% SDS-PAGE gel showing samples from the supernatant $(\mathrm{S})$, pellet $(\mathrm{P})$, flow-through after incubation (FT) and elution fractions from glutathione sepharose beads.

To improve the solubility of Rab33B, it was truncated at both its $\mathrm{N}$ - and C-termini to produce a fragment corresponding to residues 30-202, a construct that was successfully crystallized previously (Eathiraj 2005). This construct was cloned as an N-terminal Histag protein in the pETDuet-1 plasmid with a thrombin cleavage site located after the Histag which was introduced with primers during PCR. In comparison to full-length Rab33BQ92L, the truncated Rab33B(30-202)Q92L was very soluble and could be purified in high amounts, yielding $\sim 25 \mathrm{mg}$ per liter culture (Figure 3.25). 


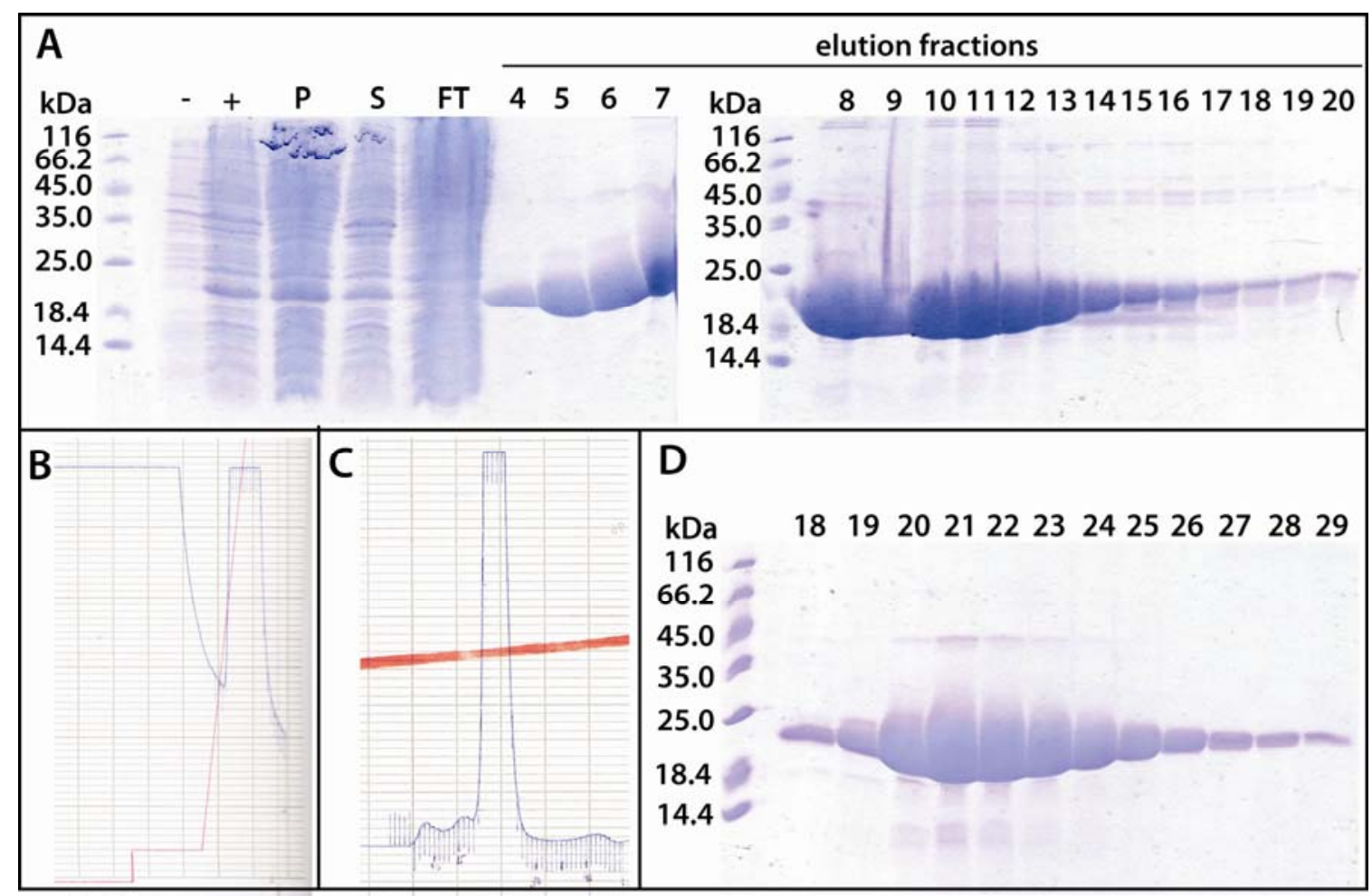

Figure 3.25: Purification of murine truncated Rab33B(30-202)Q92L. (A) 15\% SDS-PAGE gels of IMAC purification. (B) Chromatogram of HisTrap FF (two connected $1 \mathrm{~mL}$ columns) purification of HisRab33B(30-202)Q92L. Red line= \% elution buffer (C) Chromatogram of size exclusion chromatography using a HiLoad 16/60 Superdex 75 column. (D) 15\% SDS-PAGE gel of elution fractions from gel filtration purification. - = uninduced, += induced, $\mathrm{S}=$ supernatant, $\mathrm{P}=$ pellet, $\mathrm{FT}=$ flow-through, blue line $=\mathrm{UV}_{280}$ curve.

\subsubsection{Complex formation of Atg5 Atg16L1 and Rab33BQ92L}

\section{Analytical gel filtration}

To determine whether Rab33B(30-202) Q92L could still interact with Atg16L1 when it forms a complex with Atg5, an excess amount of Rab33B(30-202)Q92L was mixed with Atg5 Atg16L1(1-265) and the sample was loaded onto a Superdex 200 10/300 GL analytical gel filtration column. Co-elution of the three proteins would indicate an interaction between Rab33B(30-202)Q92L and Atg16L1(1-265) Atg5. Complex formation was indeed observed (Figure 3.26 panels A and D). Rab33B(30-202)Q92L on its own eluted with a peak at $17.5 \mathrm{~mL}$ (Figure 3.26 panel C). In contrast, Rab33B(30202)Q92L co-eluted with Atg5 Atg16L1(1-265) with the peak eluting at $10 \mathrm{~mL}$ as shown in the gels in panel D of Figure 3.26. These data demonstrate ternary complex formation between Rab33B(30-202)Q92L and Atg5 Atg16L1(1-265) and that Atg16L1 binding to Rab33B and Atg5 is not mutually exclusive. 


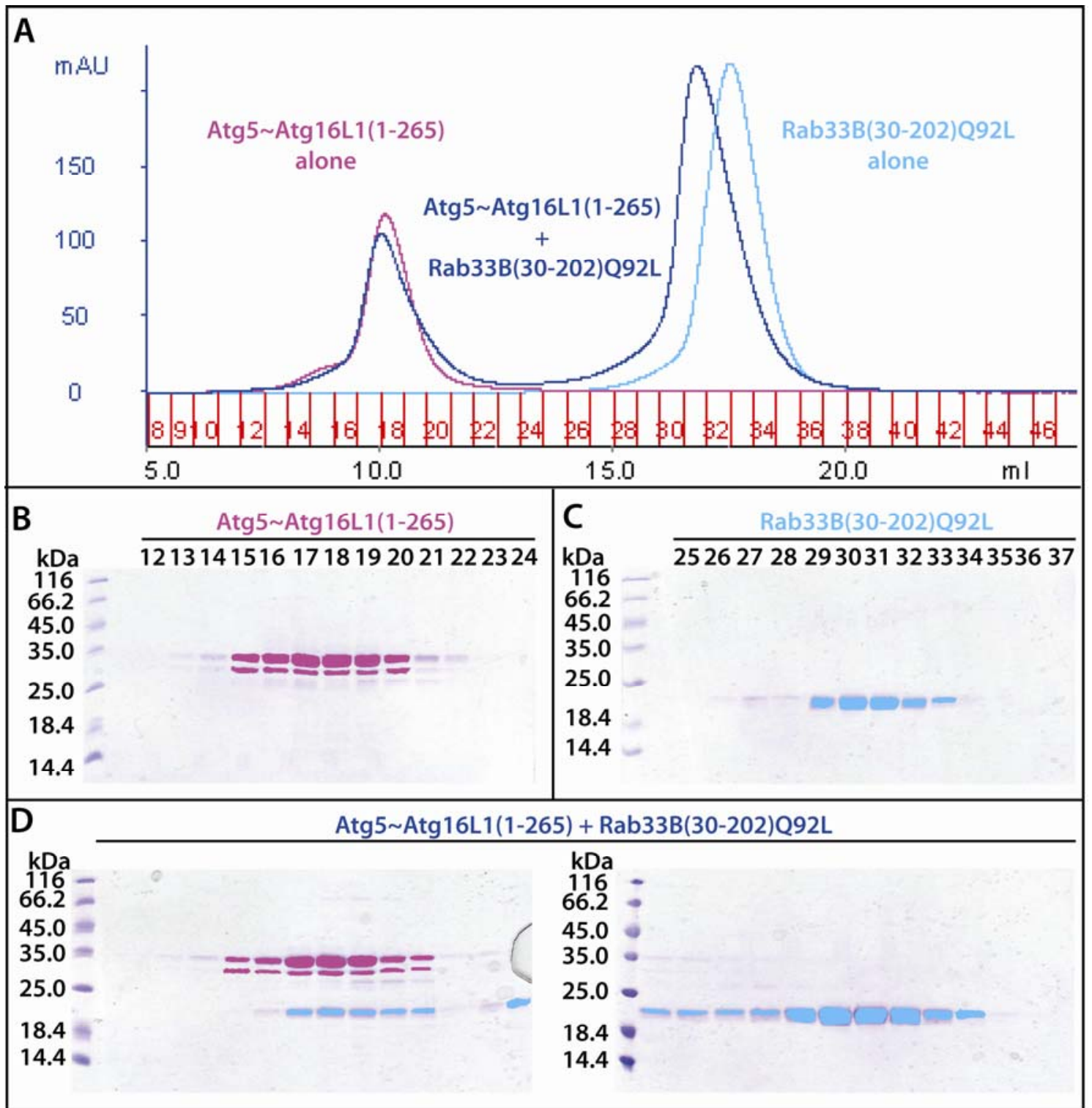

Figure 3.26: Analytical gel filtration of Rab33B(30-202) Q92L and the Atg5 Atg16L1(1-265) complex. (A) Overlay of the chromatograms from runs of the Atg5 Atg16L1(1-265) complex on its own, Rab33B(30-202)Q92L on its own, and the Atg5 Atg16L1(1-265) complex mixed with a molar excess of Rab33B(30-202)Q92L. A Superdex 200 10/300 GL analytical gel filtration column was used with running buffer composed of $0.3 \mathrm{M} \mathrm{NaCl}, 30 \mathrm{mM}$ Hepes $\mathrm{pH} 7.5,1 \mathrm{mM} \mathrm{MgCl}, 2 \mathrm{mM} \beta$-mercaptoethanol, $10 \mu \mathrm{M}$ GTP. $15 \%$ SDS-PAGE gels of elution fractions from (B) the Atg5 Atg16L1(1-265) complex run alone, (C) Rab33B(30-202)Q92L run alone, and (D) the Atg5 Atg16L1(1-265) complex and Rab33B(30-202)Q92L run together. Protein bands on gels are artificially colored to represent the indicated proteins.

\section{Isothermal titration calorimetry}

To further confirm the interaction between $\operatorname{Rab33B}(30-202) \mathrm{Q} 92 \mathrm{~L}$ and the Atg5 Atg16L1(1-265) complex and to quantify the affinity and stoichiometry of complex formation, isothermal titration calorimetry experiments were performed. When Rab33B(30-202)Q92L was titrated into the cell containing the Atg5 Atg16L1(1-265) complex, an exothermic reaction occurred (Figure 3.27 panel A). The calculated dissociation constant is $\sim 150 \mathrm{nM}$. When $\operatorname{Rab33B}(30-202) \mathrm{Q} 92 \mathrm{~L}$ was titrated into the cell 
containing a shorter fragment of Atg16L1 comprising residues 1-231 in complex with Atg5, the binding affinity decreased $\left(\mathrm{K}_{\mathrm{D}}=\sim 450 \mathrm{nM}\right)$ (Figure 3.27 panel $\left.\mathrm{B}\right)$. As shown in section 3.2.3, residues 235-265 of Atg16L1 are not required for an interaction with Rab33B(30-202)Q92L to occur. This shows us that overall, the interaction between Atg16L1 and Rab33B is in the range of medium to high affinity. The same stoichiometry of Rab33B to Atg16L1 was observed in both cases: 1 molecule of Rab33B(30-202)Q92L bound to 1 molecule of the Atg5 Atg16L1 complex. The values given are approximations.

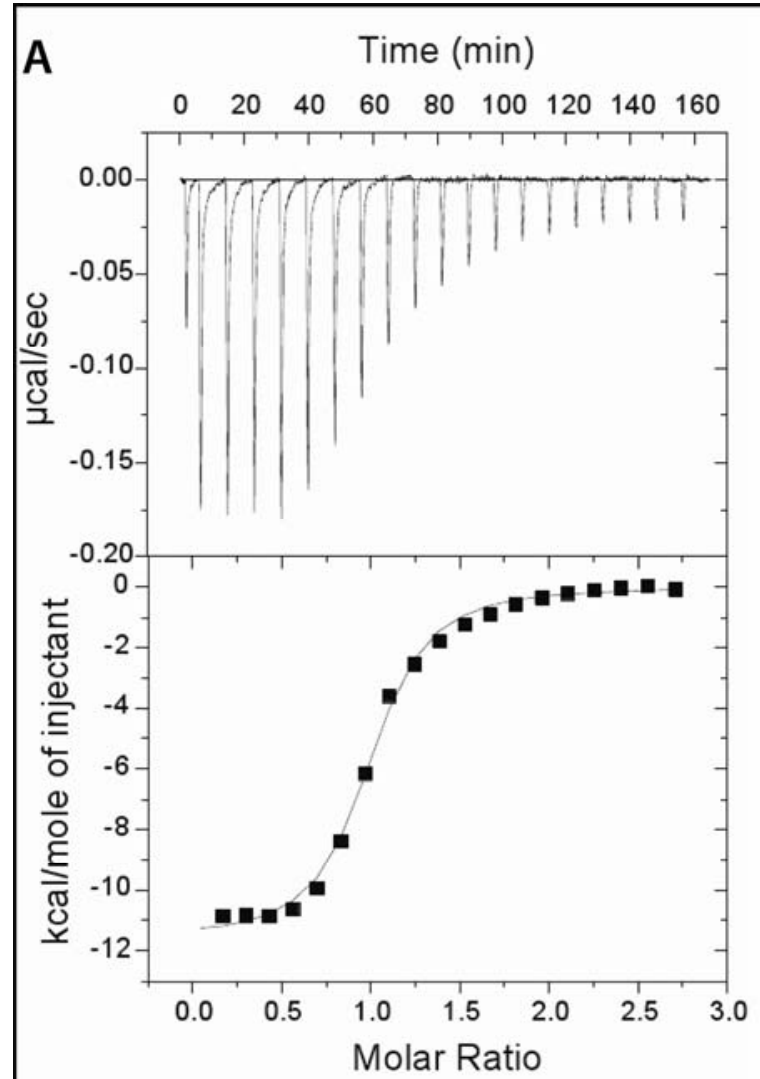

\section{B Time $(\mathrm{min})$}

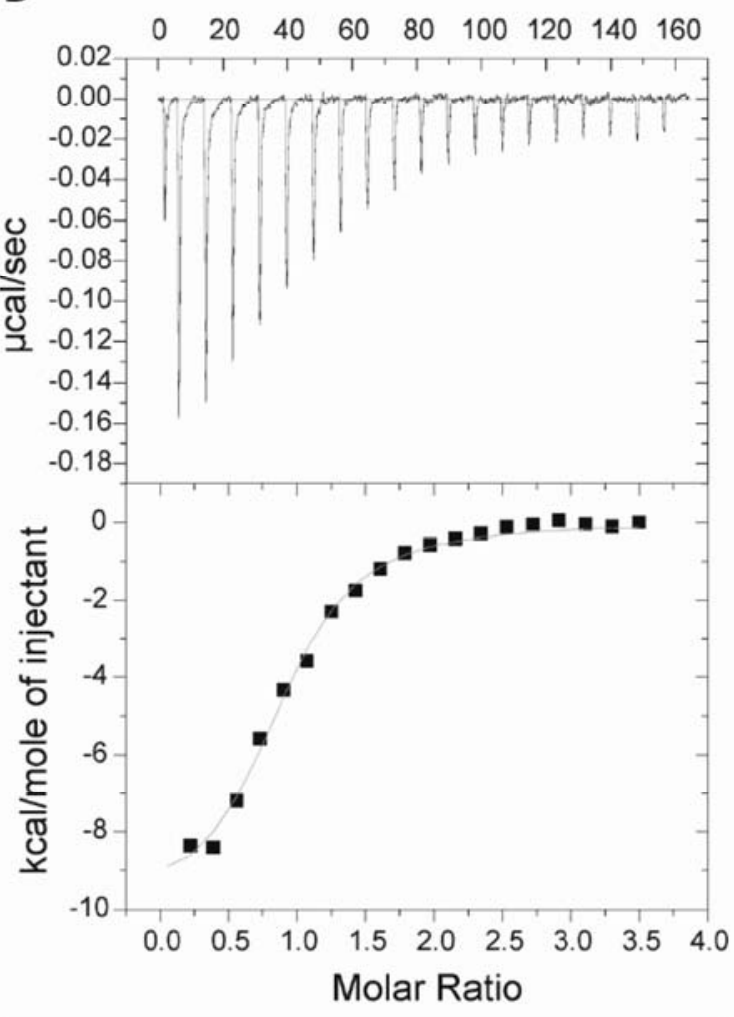

Atg5 Atg16L1(1-265) +

Rab33B(30-202)Q92L

$\mathrm{N}=0.961$

$\mathrm{Kd}=139 \mathrm{nM}$

$\Delta \mathrm{H}=-1.162 \times 10^{4} \mathrm{kcal} / \mathrm{mole}$

\section{Atg5 Atg16L1(1-231)+ Rab33B(30-202)Q92L \\ $\mathrm{N}=0.872$ \\ $\mathrm{Kd}=431 \mathrm{nM}$ \\ $\Delta \mathrm{H}=-1.007 \times 10^{4} \mathrm{kcal} / \mathrm{mole}$}

Figure 3.27: Isothermal titration calorimetry titration curves of Rab33B(30-202)Q92L and the Atg5 Atg16L1 complexes. Isothermal titration calorimetry experiments of Rab33B(30-202)Q92L with (A) Atg5 Atg16L1(1-265) and (B) Atg5 Atg16L1(1-231) are shown. 


\subsubsection{Co-purification of Rab33B Q92L with Atg16L1 constructs}

It was shown by the Fukuda lab that the C-terminal portion of the Atg16L1 coiled coil domain (residues 141-265) is responsible for binding to Rab33B (Itoh 2008). To investigate further the Rab33B-binding site of Atg16L1, three truncated Atg16L1 constructs $(172-265,203-265,172-234)$ were prepared (Figure 3.28 panel A). These constructs of Atg16L1 were co-expressed with Rab33B(30-202)Q92L and purified with Ni-sepharose beads (Figure 3.28 panel B). The Atg16L1 constructs were untagged since they were expressed from multiple cloning site 2 of the pETDuet-1 plasmid. Only if they bound to His-Rab33B(30-202)Q92L, would they co-elute with the GTPase from the Nisepharose beads. The longest Atg16L1 construct comprising the published Rab33Bbinding site (residues 141-265) co-eluted with His-Rab33B(30-202)Q92 as did the shorter Atg16L1 fragments comprising residues 172-265 and 172-234. In contrast, Atg16L1(203265) did not interact with Rab33B (Figure 3.28 panel B).
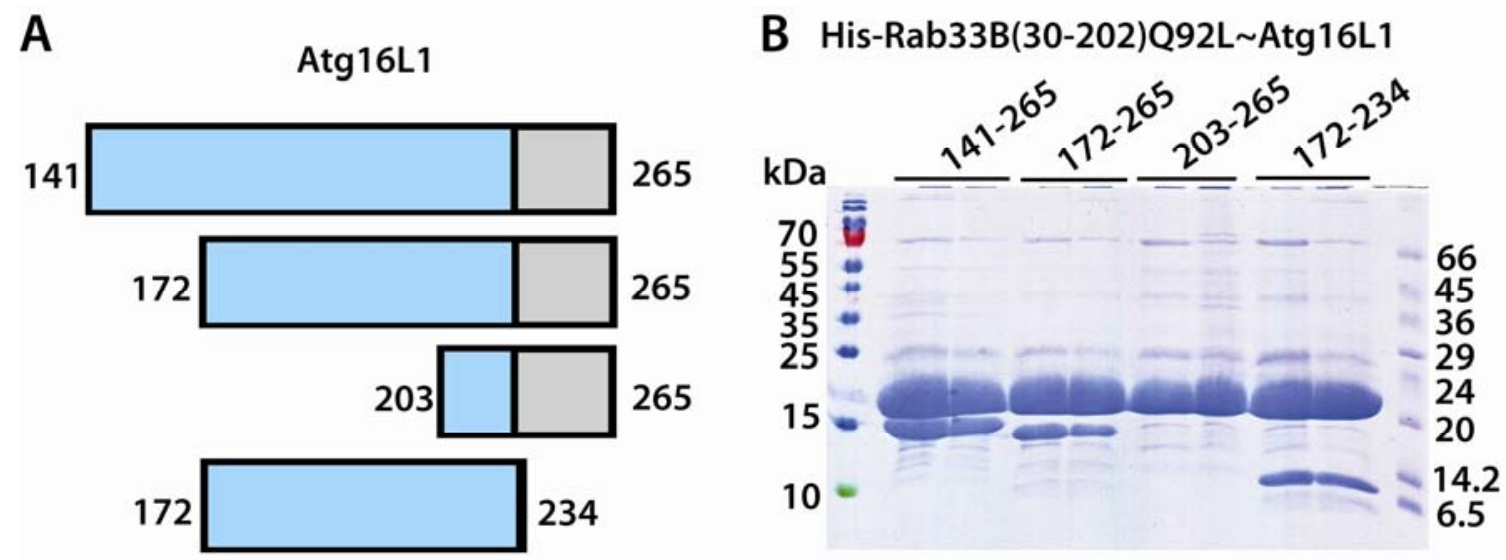

Figure 3.28: Co-expression of Atg16L1 with His-Rab33B(30-202)Q92L. (A) Atg16L1 coiled coil constructs tested for interaction with His-Rab33B(30-202)Q92L. (B) 15\% SDS-PAGE gel of elution fractions from a Ni-sepharose beads pulldown of untagged Atg16L1 constructs with His-Rab33B(30-202)Q92L.

Purifications of Rab33B(30-202)Q92L Atg16L1(141-265), Rab33B(30202)Q92L Atg16L1(172-265), and Rab33B(30-202)Q92L Atg16L1(172-234) were then up-scaled to generate sufficient amounts for crystallization trials. The complexes were first purified by IMAC followed by a polishing step with size exclusion chromatography using a HiLoad 16/60 Superdex 75 column. As seen in Figure 3.29, the Rab33B(30202)Q92L Atg16L1 complexes eluted first from the gel filtration column followed by a second peak corresponding to unbound Rab33B(30-202)Q92L. 


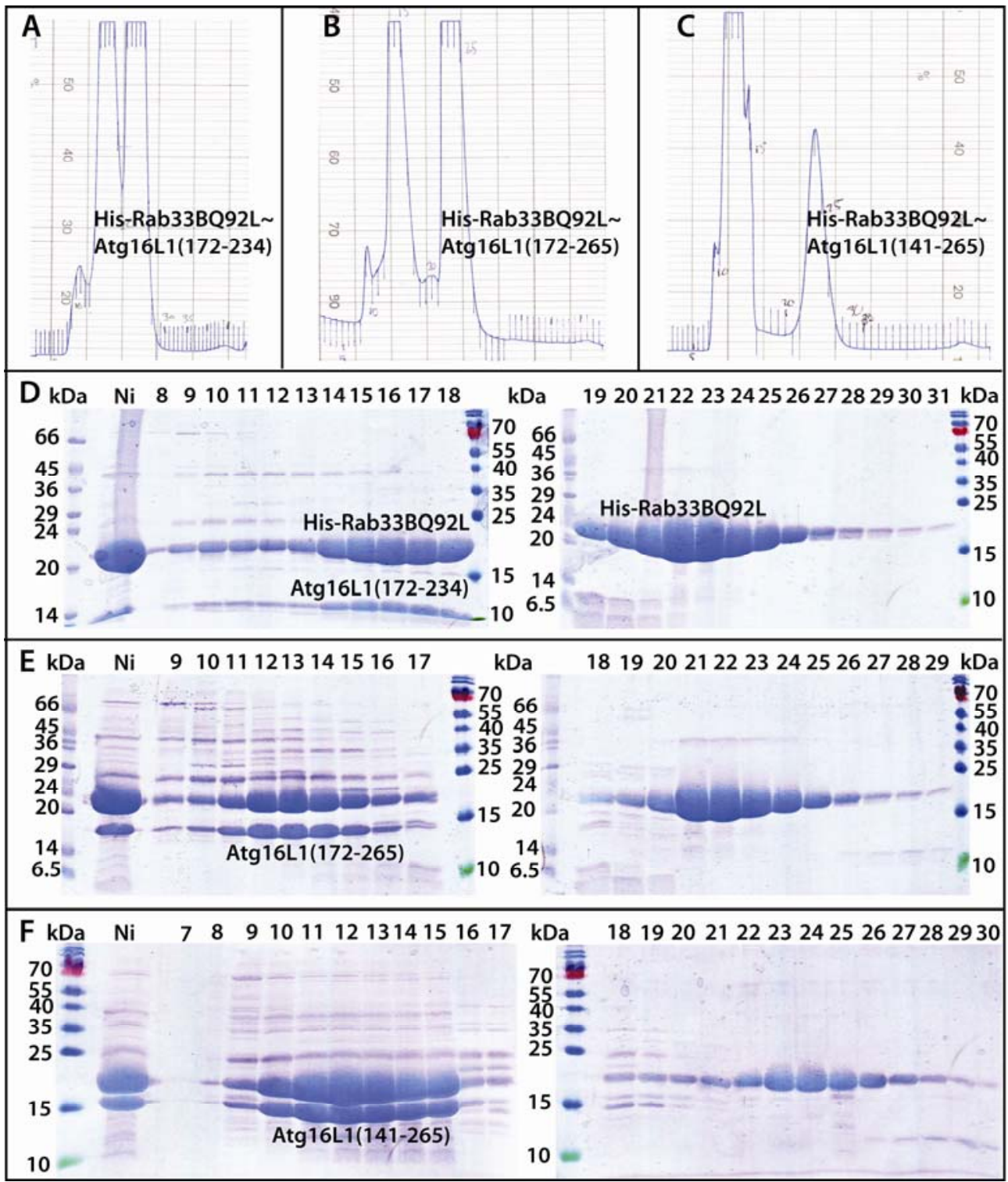

Figure 3.29: Gel filtration purifications of the Rab33B(30-202)Q92L Atg16L1 complexes. Chromatograms of size exclusion chromatography using a HiLoad 16/60 Superdex 75 column for complexes of Rab33B(30-202)Q92L with (A) Atg16L1(172-234), (B) Atg16L1(172-265), and (C) Atg16L1(141-265). 15\% SDS-PAGE gels of gel filtration runs from (D) Rab33B(30-202)Q92L Atg16L1(172234), (E) Rab33B(30-202)Q92L Atg16L1(172-265), and (F) Rab33B(30-202)Q92L Atg16L1(141-265). $\mathrm{Ni}=$ sample from pooled fractions after IMAC.

\subsubsection{Limited proteolysis experiments of Rab33B Q92 L Atg16L1 complexes}

In addition to the three constructs of Atg16L1 comprising different portions of the Rab33B-binding domain, it was attempted to identify smaller, stable fragments of Atg16L1 to use for co-crystallization with Rab33B(30-202)Q92L. Limited proteolysis was performed on Rab33B(30-202)Q92L and the Atg5 Atg16L1(1-265) complex both separately and as a ternary complex using trypsin, chymotrypsin, elastase, and proteinase K (Figure 3.30). However there was no discernable, single band that most likely corresponded to $\operatorname{Atg} 16 \mathrm{~L} 1$. 

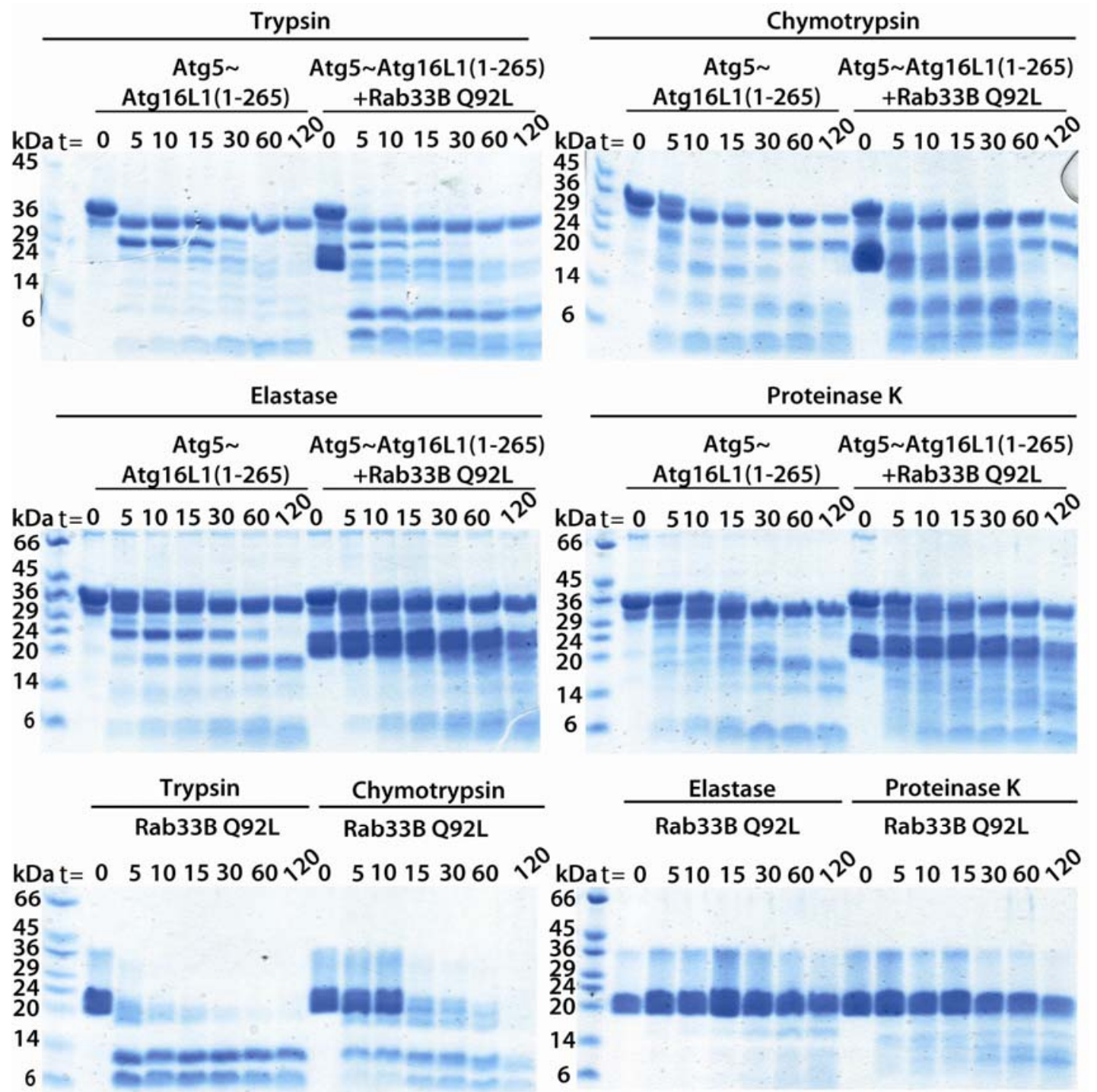

Figure 3.30: Limited proteolysis experiments of the Atg5 Atg16L1(1-265) complex and Rab33B(30-202)Q92L. Schägger Jagow gels showing the digestions of Rab33B(30-202)Q92L and the Atg5 Atg16L1(1-265) complex, separately and as a ternary complex, at RT with trypsin, chymotrypsin, and elastase at a ratio of 1:200 (w/w) and proteinase $K$ at a ratio of 1:2000 $(\mathrm{w} / \mathrm{w}) . \mathrm{t}=\mathrm{min}$.

Due to the inability to distinguish single bands of Atg16L1 from the proteolysis experiments even when using Schägger Jagow gels, which are suitable for resolving proteins smaller than $10 \mathrm{kDa}$, ion exchange chromatography was utilized to purify the proteolytic fragments. The intact ternary complex did not bind to the cation exchange Mono S 5/50 GL column using $20 \mathrm{mM}$ MES buffer at $\mathrm{pH}$ 6.0; yet after undergoing digestion with trypsin at a ratio of 1:200 (w/w), a fragment of Rab33B co-eluted with a $\sim 30 \mathrm{kDa}$ fragment which could be either Atg5 or Atg16L1 (Figure 3.31 panels $\mathrm{C}$ and E). However, when using an anion exchange column (Mono Q 5/50 GL) with $20 \mathrm{mM}$ Tris pH 8.0, intact Rab33B(30-202)Q92L no longer co-eluted with the Atg5 Atg16L1(1-265) complex. The Atg5 Atg16L1(1-265) complex eluted alone from the anion exchange 
column (Figure 3.31 panels $A$ and B). In contrast, when the Atg5 Atg16L1(1265) Rab33B(30-202)Q92L complex was digested with trypsin at a ratio of 1:200 (w/w), Rab33B(30-202)Q92L was retained on the Mono Q 5/50 GL column and co-eluted with a $\sim 30 \mathrm{kDa}$ fragment which could be Atg5 or Atg16L1 (Figure 3.31 panels A and B).

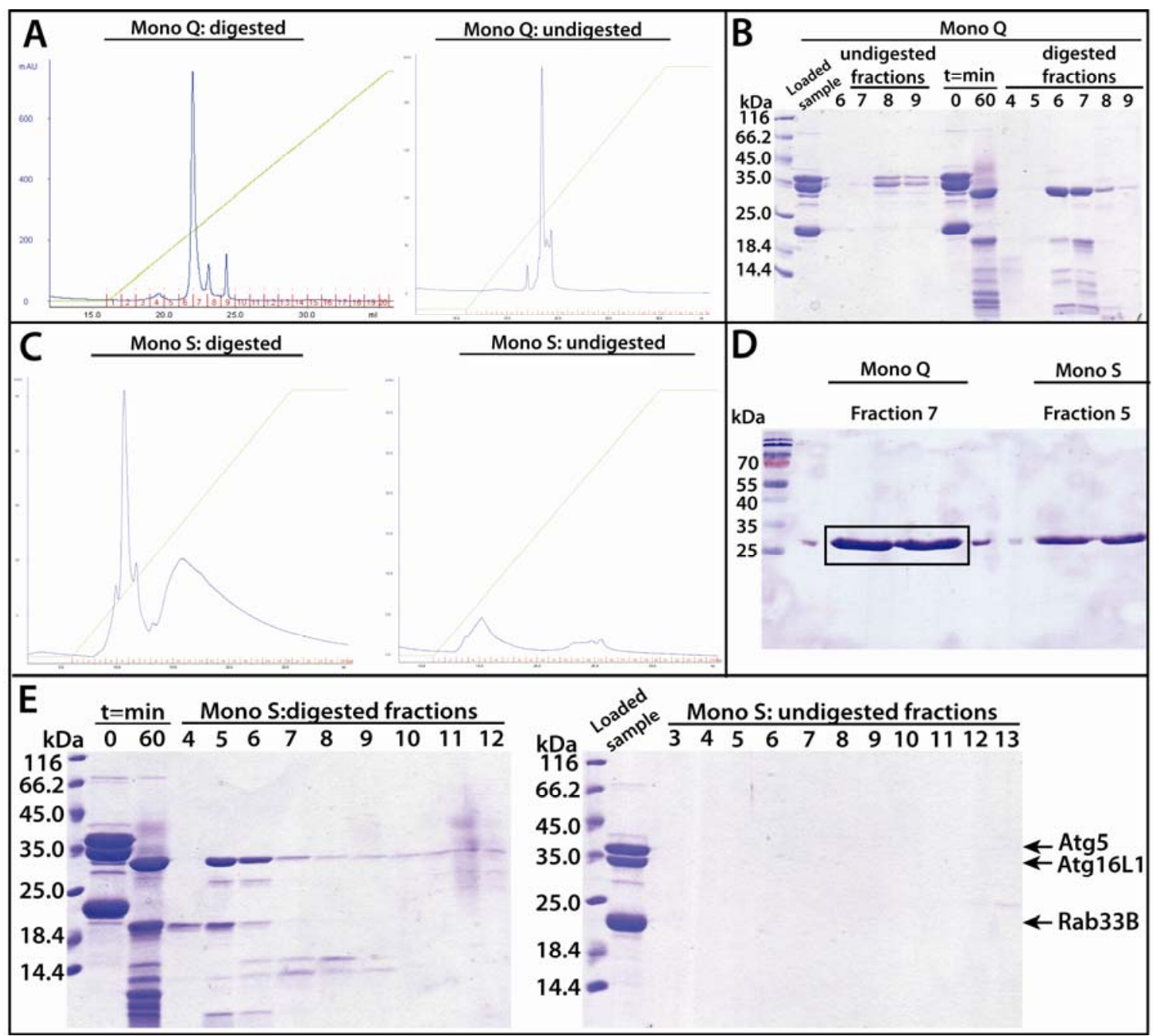

Figure 3.31: Ion exchange chromatography of the Rab33B(30-202)Q92L Atg16L1(1-265) Atg5 complex undigested and digested with trypsin. The Rab33B(20-202)Q92L Atg16L1(1-265) Atg5 complex was incubated in the absence or presence of trypsin at a ratio of 1:200 $(\mathrm{w} / \mathrm{w})$ for $60 \mathrm{~min}$ before the reaction was stopped with Pefabloc. The digested samples were diluted in binding buffer (Mono $\mathrm{Q}=20 \mathrm{mM}$ Tris pH 8.0; Mono $\mathrm{S}=20 \mathrm{mM}$ MES pH 6.0). (A) Chromatograms and (B) $15 \%$ SDS-PAGE gels of elution fractions (elution buffer=binding buffer supplemented with $1 \mathrm{M} \mathrm{NaCl}$ ) from a Mono Q 5/50 GL column. (C) Chromatograms from a Mono S 5/50 GL column. 15\% SDS-PAGE gels of (D) boxed band from the fraction transferred to PVDF and sent for $\mathrm{N}$-terminal sequencing and (E) elution fractions from a Mono $Q 5 / 50 \mathrm{GL}$ column. $\mathrm{t}=\mathrm{min}$. Blue line $=\mathrm{UV}_{280}$, green line $=\%$ elution buffer.

To identify this fragment, the reaction was repeated with more protein and the sample was immobilized on PVDF membrane and sent for N-terminal sequencing (Figure 3.31 panel C). The fragment sent for sequencing corresponded to the residues X-S-M-T (X could not be identified) which matched the thrombin cleavage site of Atg5. The smaller 
$\sim 10 \mathrm{kDa}$ band which eluted from the Mono $\mathrm{S}$ column was sent for $\mathrm{N}$-terminal sequencing (Figure 3.32). The four amino acids determined by N-terminal sequencing, R-G-E-L, match residues 103 to 106 of murine Atg16L1. This would yield a fragment ending approximately between residues 200 and 215 .

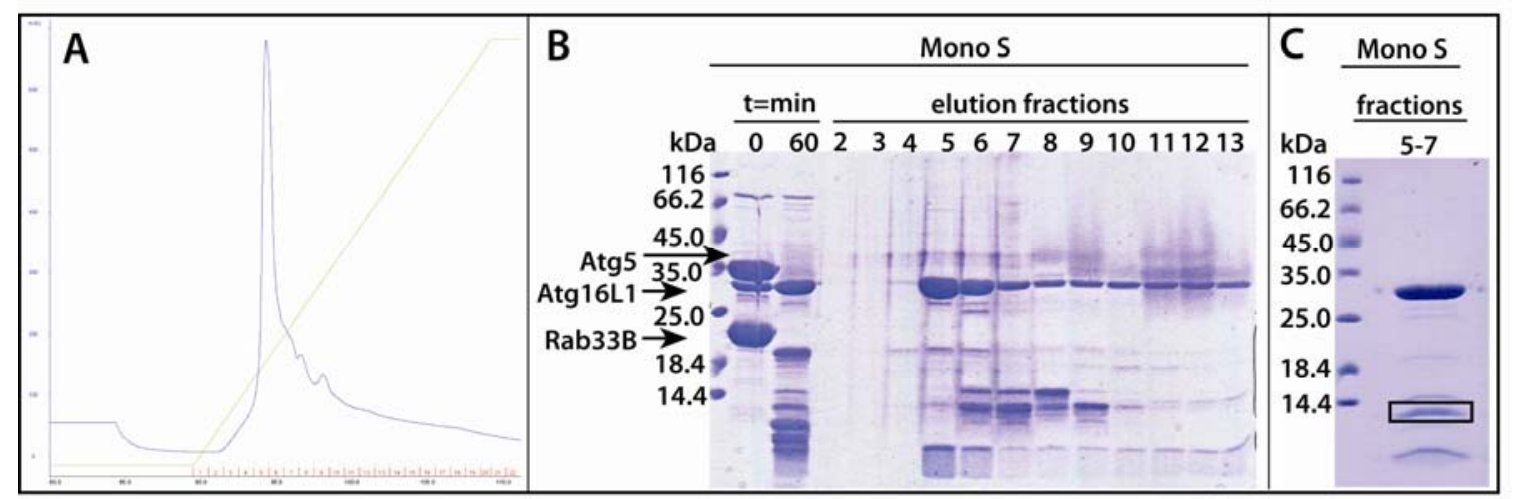

Figure 3.32: Cation exchange chromatography of the ternary Rab33B(30-202)Q92L Atg16L1(1265) Atg 5 complex digested with trypsin and the sample sent for $\mathbf{N}$-terminal sequencing. The Atg5 Atg16L1(1-265) complex together with Rab33B(20-202)Q92L was incubated in the presence of trypsin at a ratio of 1:200 (w/w) for 60 min before the reaction was stopped with Pefabloc. The digested sample was loaded onto a Mono S 5/50 GL cation exchange column.

(A) Chromatogram of the run. Blue line $=U V_{280}$, green line $=\%$ elution buffer. $15 \%$ SDS-PAGE gels of $(B)$ the digestion and elution fractions and $(C)$ pooled fractions 5 to 7 with the boxed band having been blotted onto PVDF membrane and sent for $\mathrm{N}$-terminal sequencing. Binding buffer $=20 \mathrm{mM} \mathrm{MES} \mathrm{pH} \mathrm{6.0.} \mathrm{Elution} \mathrm{buffer}=20 \mathrm{mM} \mathrm{MES} \mathrm{pH}$ $6.0,1 \mathrm{M} \mathrm{NaCl}$.

Since a truncated Atg16L1 fragment could not be identified by digestion of the ternary $\operatorname{Rab33B(30-202)Q92L\sim Atg16L1\sim Atg5~complex,~limited~proteolysis~of~the~}$ Rab33B(30-202)Q92L Atg16L1(141-265) was also carried out. The Rab33B(30202)Q92L Atg16L1(141-265) complex was digested with trypsin and chymotrypsin at a ratio of 1:200 (w/w) (Figure 3.33). Several $\sim 10 \mathrm{kDa}$ cleavage products were formed during the digestions of both the Rab33B(30-202)Q92L Atg16L1(141-265) complex and Rab33B(30-202)Q92L alone. This presented a challenge to the identification of an Atg16L1 fragment. Therefore the purification of the digestion products through ion exchange chromatography was tested.

Rab33B(30-202)Q92L alone and the Rab33B(30-202)Q92L Atg16L1(141-265) complex were digested with trypsin at a ratio of 1:200 (w/w) and the reaction was stopped with Pefabloc after $5 \mathrm{~min}$ incubation at room temperature. The digested samples were loaded onto both cation (Mono S 5/50 GL, MES pH 6.0) (Figure 3.34) and anion (Mono Q 5/50 GL, Tris pH 8.0) (Figure 3.35) exchange columns. Only one band corresponding to Rab33B eluted from the Mono S column (Fig. 3.34 panel B) after digestion of the 
Rab33BQ92L Atg16L1 complex. However, several bands co-eluted from the Mono Q column, enabling separation of the digested Rab33BQ92L Atg16L1 complex (Figure 3.35 panel B). These bands were not observed when digested Rab33B(30-202)Q92L alone was loaded onto the Mono Q column (Fig. 3.35 panel B).

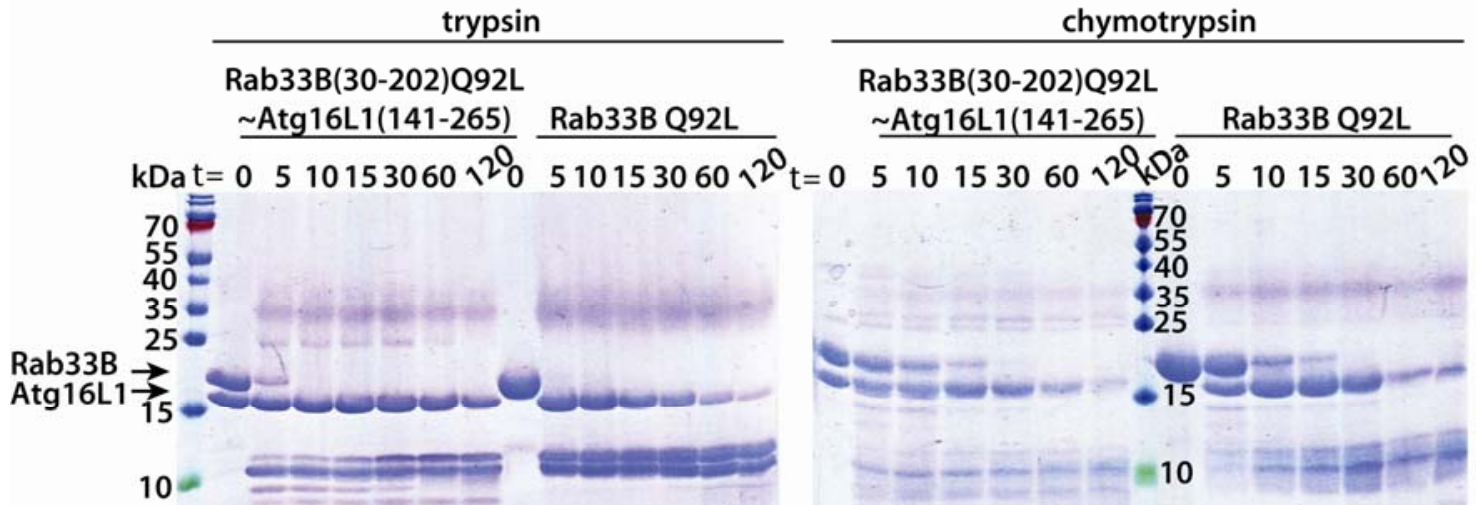

Figure 3.33: Limited proteolysis of the Rab33BQ92L Atg16L1(141-265) complex. 15\% SDS-PAGE gels of digestion of the Rab33B(30-202)Q92L Atg16L1(141-265) complex and Rab33B(30-202)Q92L alone with trypsin and chymotrypsin, both at a ratio of 1:200 (w/w). $t=\mathrm{min}$.

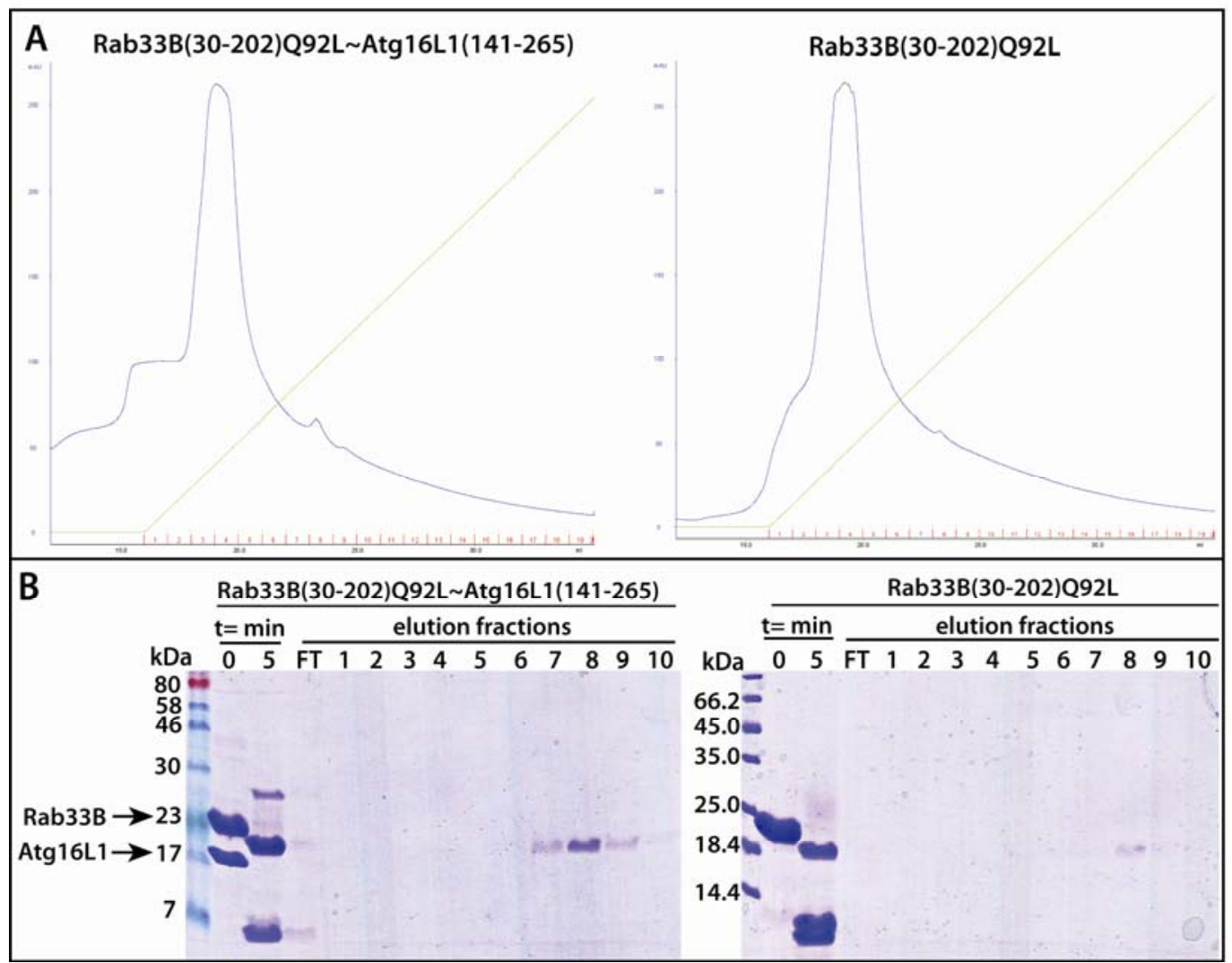

Figure 3.34: Cation exchange chromatography of the Rab33B(30-202)Q92L Atg16L1(141-265) complex and Rab33B(30-202)Q92L alone digested by trypsin. The Rab33B(20-202)Q92L Atg16L1(1265) complex and Rab33B(20-202)Q92L alone were incubated in the presence of trypsin at a ratio of 1:200 (w/w) for $5 \mathrm{~min}$ at RT before the reaction was stopped with Pefabloc. (A) Chromatograms of cation exchange chromatography using a Mono S 5/50 GL column. (B) 15\% SDS-PAGE gels of elution fractions. $\mathrm{FT}=$ flow-through, blue line $=\mathrm{UV}_{280}$, green line $=\%$ elution buffer $(20 \mathrm{mM} \mathrm{MES} \mathrm{pH} \mathrm{6.0,} 1 \mathrm{M} \mathrm{NaCl})$. 


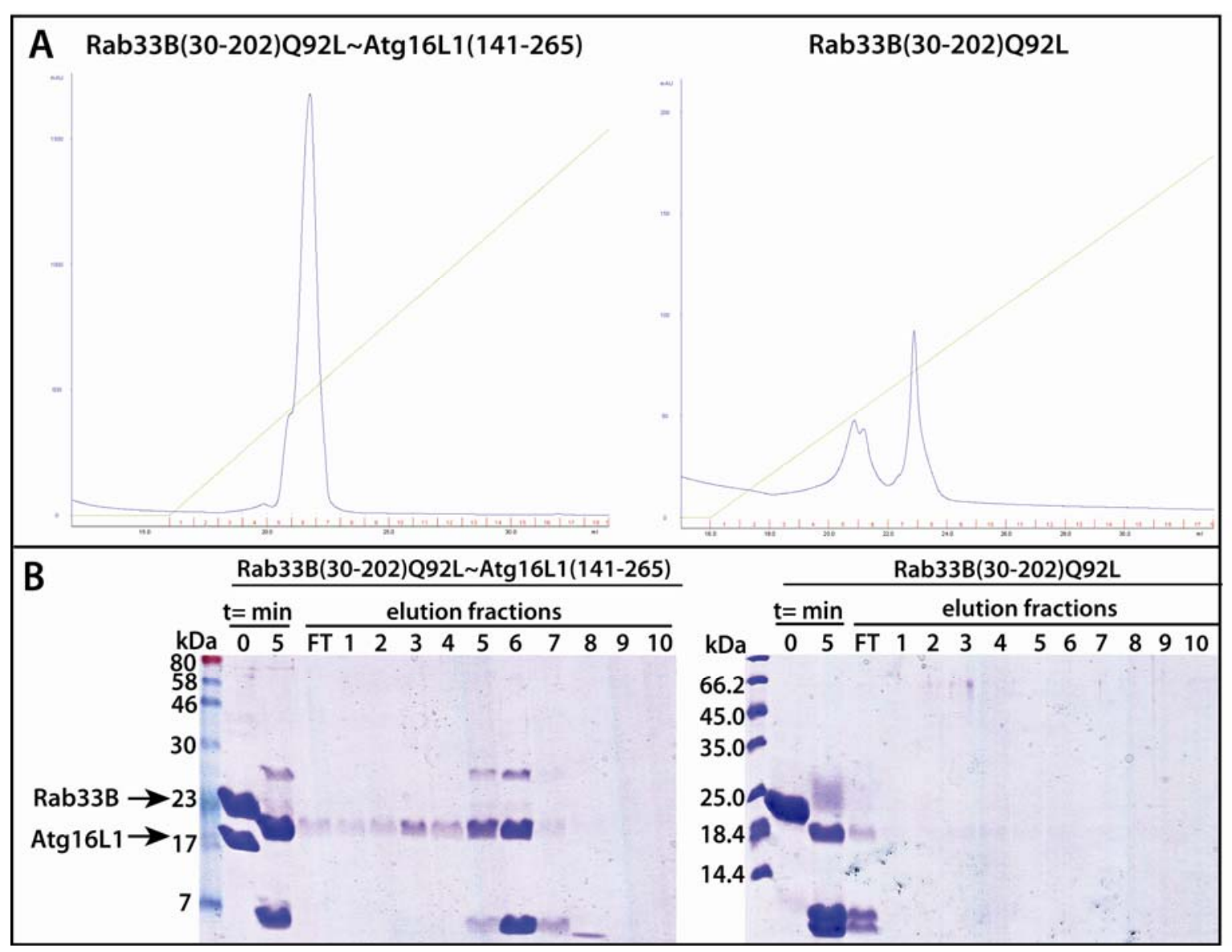

Figure 3.35: Anion exchange chromatography of the Rab33B(30-202)Q92L Atg16L1(141-265) complex and Rab33B(30-202)Q92L alone digested by trypsin. The Rab33B(20-202)Q92L Atg16L1(1265) complex and Rab33B(20-202)Q92L alone were incubated in the presence of trypsin at a ratio of 1:200 (w/w) for 5 min at RT before the reaction was stopped with Pefabloc. (A) Chromatograms of anion exchange chromatography using a Mono Q 5/50 GL column. Blue line $=\mathrm{UV}_{280}$, green line $=\%$ elution buffer (20 mM Tris pH 8.0, $1 \mathrm{M} \mathrm{NaCl}$ ). (B) 15\% SDS-PAGE gels of elution fractions. FT = flow-through.

A sample of the digested Rab33B(30-202)Q92L Atg16L1(141-265) complex that eluted from the Mono Q 5/50 GL column was immobilized onto PVDF membrane and was sent off for N-terminal sequencing (shown boxed in Fig. 3.36). As can be best seen in the Schägger Jagow gel in Figure 3.35 panel B, this band corresponds to a MW of approximately 7 to $10 \mathrm{kDa}$. The first four amino acids of this fragment were M-L-E-T which corresponds to Atg16L1 residues 141-143 with the methionine residue at the Nterminus having been introduced via the NdeI restriction site during cloning into the second multiple cloning site of the pETDuet-1 plasmid. Since the N-terminus of Atg16L1 was intact, this would correspond to a fragment ending approximately in the range $\sim 195$ 200 in the Atg16L1 sequence. 

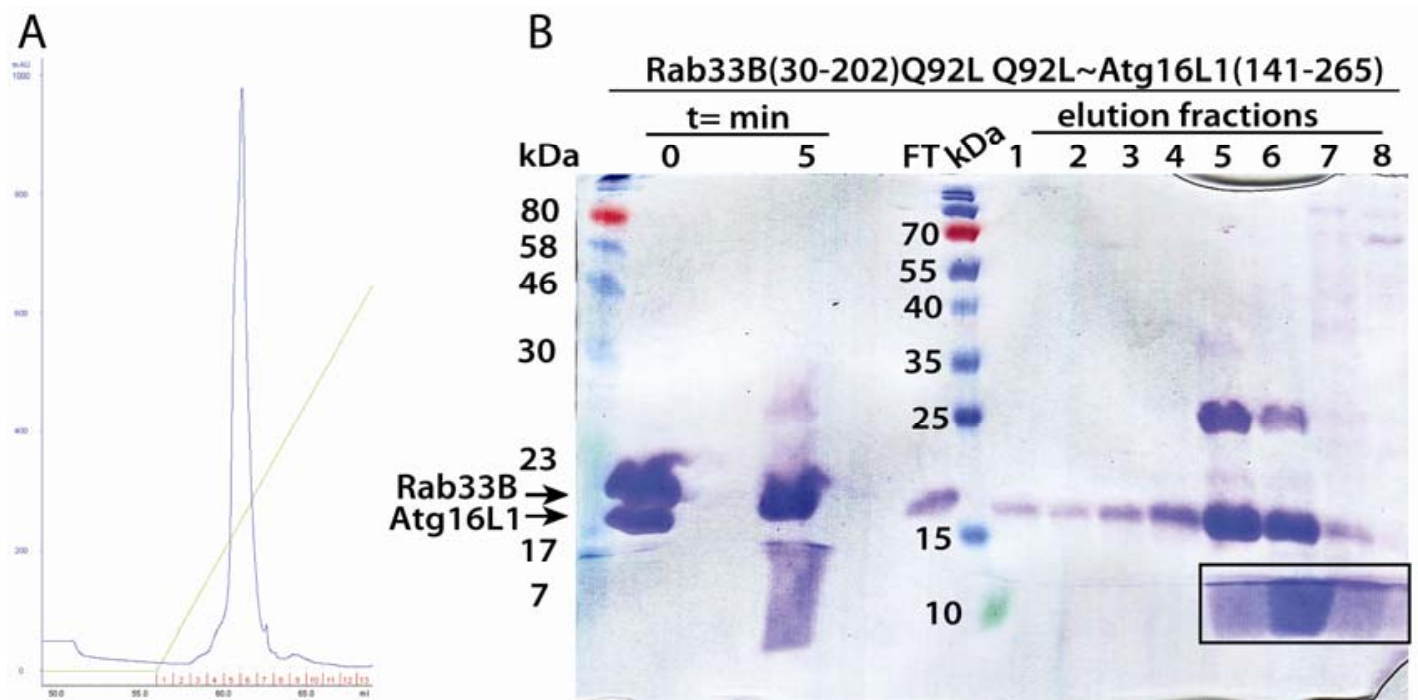

Figure 3.36: Anion exchange chromatography of the Rab33B(30-202)Q92L Atg16L1(141-265) complex digested with trypsin. The Rab33B(20-202)Q92L Atg16L1(141-265) complex was incubated in the presence of trypsin at a ratio of 1:200 (w/w) for $5 \mathrm{~min}$ at RT before the reaction was stopped with Pefabloc. (A) Chromatogram of the digested complex run on a Mono Q 5/50 GL column (20 mM Tris pH 8.0). Blue line $=\mathrm{UV}_{280}$, green line $=\%$ elution buffer ( $20 \mathrm{mM}$ Tris $\mathrm{pH}$ 8.0, $1 \mathrm{M} \mathrm{NaCl}$ ). (B) $15 \%$ SDS-PAGE gel of digestion and elution fractions. The box indicates the fraction later sent for $\mathrm{N}$-terminal sequencing after immobilization onto PVDF membrane.

Based on the N-terminal sequencing results and crystallization of the Rab33B(30202)Q92L Atg16L1(141-265) complex (see section 3.2.5), the construct Atg16L1(125204 was cloned. The N-terminus of Atg16L1 was extended further since residues Nterminal of position 141 could potentially be involved in Rab33B binding. The Cterminus was also extended 5-10 residues past that predicted by the digestion fragment to make sure that the complete Rab33B binding site was included. Purification of the coexpressed Rab33B(30-202)Q92L Atg16L1(125-204) complex by IMAC resulted in a single peak (Fig. 3.37 panel A). However, analysis of the elution fractions showed the protein eluted consisted not of the complex but only of His-Rab33B(30-202)Q92L on its own (Fig. 3.37 panel B). Atg16L1(125-204) is soluble and found in the flow-through fraction. This shows that no stable complex was formed between Rab33BQ92L and Atg16L1. 


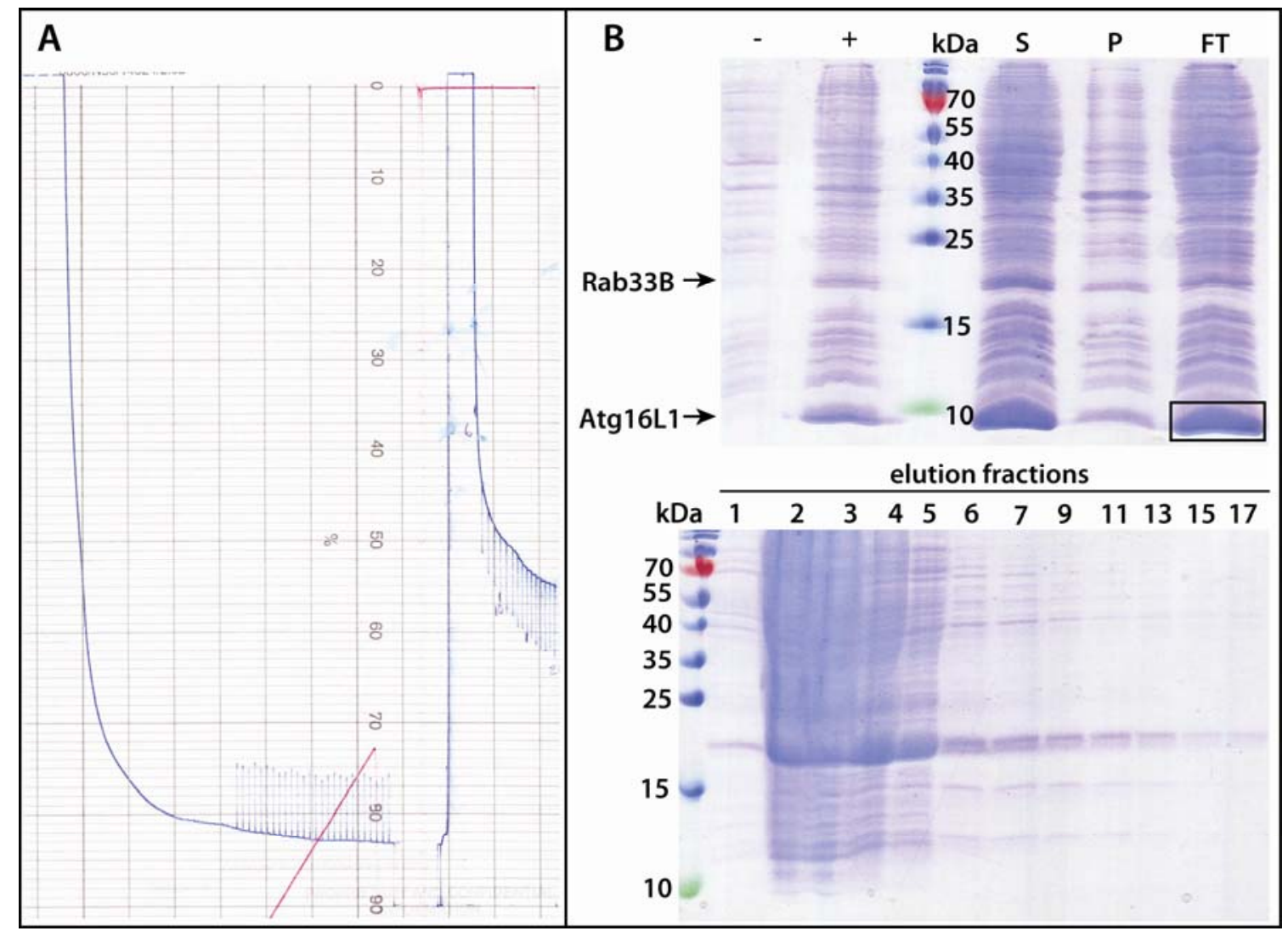

Figure 3.37: Purification of His-Rab33B(30-202)Q92L Atg16L1(125-204). (A) Chromatogram of Nisepharose column purification of co-expressed His-Rab33B(30-202)Q92L Atg16L1(125-204). (B) 15\% SDS-PAGE gels of the expression analysis showing Atg16L1 primarily in the flow-through (box) and only Rab33B(30-202)Q92L in the elution fractions. - = uninduced, + = induced, $\mathrm{S}=$ supernatant, $\mathrm{P}=$ pellet, $\mathrm{FT}=$ flow-through, blue line $=U V_{280}$ curve, red line $=\%$ elution buffer.

\subsubsection{Crystallization of $\operatorname{Rab33B(30-202)Q92L~Atg16L1~complexes~}$}

With the aim of determining the structure of a Rab33B Q92L Atg16L1 complex using x-ray crystallography, a large variety of crystallization screens were set up. The first ones consisted of the ternary complex of His-Rab33B(30-202)Q92L Atg16L1(1265) Atg5. This was followed by screens set up with the three co-purified complexes: His-Rab33B(30-202)Q92L Atg16L1(141-265), His-Rab33B(30-202)Q92L Atg16L1(172-265), and His-Rab33B(30-202) Q92L Atg16L1(172-234). Furthermore, crystallization screens were also set up with the His-Rab33B(30-202) Q92L Atg16L1(141-265) and His-Rab33B(30-202)Q92L Atg16L(1-265) Atg5 complexes after digestion with trypsin. All of these screens were set up at $20{ }^{\circ} \mathrm{C}$. Despite the various complexes and crystallization screens utilized, crystals only grew of the HisRab33B(30-202)Q92L Atg16L1(141-265) complex which took from 2 to 6 weeks.

Conditions for crystallization of the Rab33B(30-202)Q92L Atg16L1(141-265) complex were also scanned in 24 -well Linbro plates by the hanging drop method. The $\mathrm{pH}$ 
was varied from pH 6.5 to 9.0, the precipitants PEG 20000, PEG 12000, PEG 10000, and PEG 4000 were tested from $~ 12-24 \%(w / v)$, and magnesium chloride hexahydrate was added in some of the screens. The concentration of the protein tested in the 24-well Linbro plates ranged from 15 to $45 \mathrm{mg} / \mathrm{mL}$. Attempts at streak seeding did not yield crystals. The very few crystals that grew in the optimized conditions grew very slowly, taking up to four months to grow. Since crystals were not easily reproduced in the 24-well Linbro plates, several factors were scanned in the 96-well Greiner and MRC plates by the sitting drop method using the screens from Qiagen that had produced initial hits. The protein concentration was tested from 5 to $55 \mathrm{mg} / \mathrm{mL}$ and additional screens were set up and stored at $4{ }^{\circ} \mathrm{C}$. Crystallization conditions of the Rab33B(30-202) Q92L Atg16L1(141-265) complex (Figure 3.38) are summarized in Table 3.9.

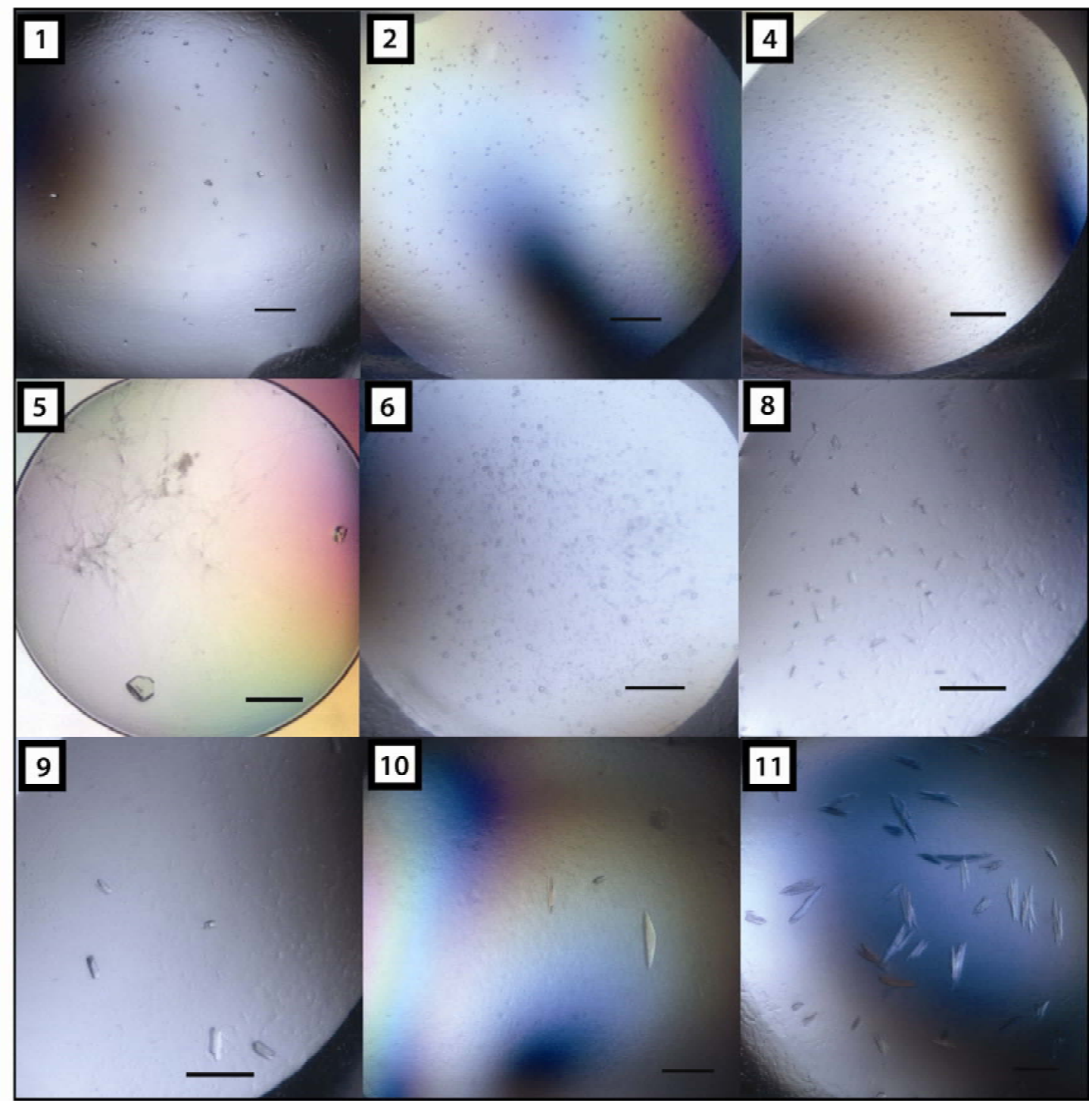

Figure 3.38: Crystals of the murine His-Rab33B(30-202)Q92L Atg16L1(141-265) complex. Numbers correspond to the crystallization conditions in Table 3.9. Scale bar $=200 \mu \mathrm{m}$. 
Crystals of murine Rab33B(30-202)Q92L Atg16L1(141-265) were equilibrated in cryoprotectants consisting of $20 \%$ PEG 400 or $25 \%$ ethylene glycol using a 3 -step serial dilution. After equilibration in cryoprotectant, the crystals were flash-cooled in liquid nitrogen. Crystals were sent to the Swiss Light Source (Switzerland) and their diffraction was tested at beamline PXII. However, these crystals did not diffract.

Table 3.9: Crystallization conditions for His-Rab33B(30-202) Q92L Atg16L1(141-265)

\begin{tabular}{|c|c|c|c|c|c|c|}
\hline & Buffer & Precipitant & Salt & $\begin{array}{l}\text { [Protein] } \\
(\mathrm{mg} / \mathrm{mL})\end{array}$ & $\begin{array}{l}\text { Drop } \\
\text { setup }\end{array}$ & Temp. \\
\hline 1 & $\begin{array}{c}0.1 \mathrm{M} \text { HEPES sodium } \\
\mathrm{pH} 7.5\end{array}$ & $30 \%(\mathrm{v} / \mathrm{v})$ isopropanol & $0.2 \mathrm{M} \mathrm{MgCl}_{2}$ & 30,20 & $\begin{array}{c}100: \\
100 \mathrm{~nL}\end{array}$ & $20^{\circ} \mathrm{C}$ \\
\hline 2 & 0.1 M HEPES pH 7.5 & $30 \%(\mathrm{v} / \mathrm{v})$ PEG $550 \mathrm{MME}$ & $0.05 \mathrm{M} \mathrm{MgCl}_{2}$ & $60,50,40$ & $\begin{array}{c}100: \\
100 \mathrm{~nL}\end{array}$ & $20^{\circ} \mathrm{C}$ \\
\hline 3 & $0.1 \mathrm{M}$ HEPES $\mathrm{pH} 7.5$ & $30 \%(\mathrm{v} / \mathrm{v})$ PEG $550 \mathrm{MME}$ & $0.05 \mathrm{M} \mathrm{MgCl}_{2}$ & 30 & $\begin{array}{c}100: \\
100 \mathrm{~nL}\end{array}$ & $20^{\circ} \mathrm{C}$ \\
\hline 4 & $0.1 \mathrm{M}$ Tris $\mathrm{pH} 8.5$ & 25\% (w/v) PEG 3350 & $0.2 \mathrm{M} \mathrm{MgCl}_{2}$ & 20,10 & $\begin{array}{c}100: \\
100 \mathrm{~nL}\end{array}$ & $20^{\circ} \mathrm{C}$ \\
\hline 5 & 0.1 M HEPES pH 7.0 & $18 \%(\mathrm{w} / \mathrm{v})$ PEG 12000 & -------------------- & 40,30 & $\begin{array}{c}100: \\
100 \mathrm{~nL}\end{array}$ & $20^{\circ} \mathrm{C}$ \\
\hline 6 & $0.1 \mathrm{M}$ HEPES $\mathrm{pH} 7.5$ & $30 \%(\mathrm{w} / \mathrm{v})$ PEG 400 & $0.2 \mathrm{M} \mathrm{MgCl}_{2}$ & 40,30 & $\begin{array}{c}100: \\
100 \mathrm{~nL}\end{array}$ & $20^{\circ} \mathrm{C}$ \\
\hline 7 & $0.1 \mathrm{M}$ Tris $\mathrm{pH} 8.5$ & $16 \%(\mathrm{w} / \mathrm{v})$ PEG 4000 & $0.2 \mathrm{M} \mathrm{MgCl}_{2}$ & 40 & $\begin{array}{c}100: \\
100 \mathrm{~nL}\end{array}$ & $20^{\circ} \mathrm{C}$ \\
\hline 8 & $\begin{array}{l}0.09 \mathrm{M} \text { HEPES } \\
\text { sodium } \mathrm{pH} 7.5\end{array}$ & $\begin{array}{c}27 \%(\mathrm{v} / \mathrm{v}) \text { isopropanol, } \\
10 \%(\mathrm{v} / \mathrm{v}) \text { glycerol }\end{array}$ & $0.18 \mathrm{M} \mathrm{MgCl}_{2}$ & 40 & $\begin{array}{c}100: \\
100 \mathrm{~nL}\end{array}$ & $20^{\circ} \mathrm{C}$ \\
\hline 9 & $\begin{array}{l}0.09 \mathrm{M} \text { HEPES } \\
\text { sodium } \mathrm{pH} 7.5 \\
\end{array}$ & $\begin{array}{c}27 \%(\mathrm{v} / \mathrm{v}) \text { isopropanol, } \\
10 \%(\mathrm{v} / \mathrm{v}) \text { glycerol }\end{array}$ & $0.18 \mathrm{M} \mathrm{MgCl}_{2}$ & 30,20 & $\begin{array}{c}100: \\
100 \mathrm{~nL}\end{array}$ & $20^{\circ} \mathrm{C}$ \\
\hline 10 & $0.1 \mathrm{M}$ Tris $\mathrm{pH} 8.5$ & $20 \%(\mathrm{w} / \mathrm{v})$ PEG 6000 & -------------------- & 50 & $\begin{array}{c}100: \\
100 \mathrm{~nL}\end{array}$ & $4^{\circ} \mathrm{C}$ \\
\hline 11 & $0.1 \mathrm{M}$ Tris $\mathrm{pH} 8.5$ & $20 \%(\mathrm{w} / \mathrm{v})$ PEG 6000 & -------------------- & 30 & $\begin{array}{c}100: \\
100 \mathrm{~nL}\end{array}$ & $4^{\circ} \mathrm{C}$ \\
\hline 12 & $0.1 \mathrm{M}$ Tris $\mathrm{pH} 8.0$ & $22 \%(\mathrm{w} / \mathrm{v})$ PEG 10000 & --------------------- & 35 & $1: 1 \mu \mathrm{L}$ & $20^{\circ} \mathrm{C}$ \\
\hline 13 & $0.1 \mathrm{M}$ Tris $\mathrm{pH} 8.0$ & 24\%(w/v) PEG 10000 & -------------------- & 40 & $1: 1 \mu \mathrm{L}$ & $20^{\circ} \mathrm{C}$ \\
\hline 14 & $0.1 \mathrm{M}$ Tris $\mathrm{pH} 8.0$ & $18 \%(\mathrm{w} / \mathrm{v})$ PEG 4000 & $0.2 \mathrm{M} \mathrm{MgCl}_{2}$ & 40 & $1: 1 \mu \mathrm{L}$ & $20^{\circ} \mathrm{C}$ \\
\hline
\end{tabular}




\subsection{Characterization of phosphoinositide binding of the}

\section{Atg5 Atg16 complex}

\subsubsection{Atg5 Atg16L1 binds phosphoinositides}

The association of the Atg12-Atg5 Atg16L1 complex with the growing isolation membrane is essential for autophagosome formation but the mechanism of recruitment of the Atg12-Atg5 Atg16 complex to the membrane is still unclear (Suzuki 2001, Mizushima 2001). To investigate whether a direct interaction of membrane lipids with the mammalian Atg5 Atg16L1 complex could occur, the Atg5 Atg16L1(1-70) complex was used as a probe in a protein lipid overlay assay with a membrane lipid strip (Echelon) which contains 15 different spotted lipids. The mammalian Atg5 Atg16L1(1-70) complex bound to phosphatidylinositol-4-phosphate (PI(4)P) (Fig. 3.39).

\section{His-Atg5 His-Atg16L1(1-70)}

$\begin{aligned} \text { Triglyceride } & \text { Phosphatidylinositol (PI) } \\ \text { Diacylglycerol } & \mathrm{PI}(4) \mathrm{P} \\ \text { Phosphatidic acid (PA) } & \mathrm{PI}(4,5) \mathrm{P}_{2} \\ \text { Phosphatidylserine (PS) } & \mathrm{PI}(3,4,5) \mathrm{P}_{3} \\ \text { Phosphatidylethanolamine (PE) } & \text { Cholesterol } \\ \text { Phosphatidylcholine (PC) } & \text { Sphingomyelin } \\ \text { Phosphatidylglycerol (PG) } & \text { 3-sulfogalactosylceramide } \\ \text { Cardiolipin } & \text { Blank }\end{aligned}$

Figure 3.39: Membrane lipid strip protein lipid overlay assay using His-Atg5 His-Atg16L1(1-70). A protein lipid overlay assay (membrane lipid strip, Echelon) was performed with the mammalian Atg5 Atg16L1(1-70) complex at a concentration of $5 \mu \mathrm{g} / \mathrm{mL}$. Binding was detected with a murine monoclonal anti-His-tag antibody (Dianova).

The phosphoinositide PI(3)P is enriched in autophagosomal membranes (Obara 2008). To further investigate the specificity of phosphoinositide binding of Atg5 Atg16L1, PIP strips (Echelon) were used. Here the mammalian Atg5 Atg16L1(170) complex interacted with both PI(3)P and PI(5)P (Fig. 3.40). The Atg5 Atg16L1(1265) complex bound all three phosphatidylinositol-monophosphates in addition to $\mathrm{PI}(3,5) \mathrm{P}_{2}$ (Fig. 3.40). 
His-Atg5 His-Atg16L1(1-70)

$\begin{array}{rl}\text { Lysophosphatidic acid } & \text { Sphingosine-1-Phosphate } \\ \text { Lysophosphocholine } & \mathrm{PI}(3,4) \mathrm{P}_{2} \\ \text { Phosphatidylinositol } & \mathrm{PI}(3,5) \mathrm{P}_{2} \\ \mathrm{PI}(3) \mathrm{P} & \mathrm{PI}(4,5) \mathrm{P}_{2} \\ \mathrm{PI}(4) \mathrm{P} & \mathrm{PI}(3,4,5) \mathrm{P}_{3} \\ \mathrm{PI}(5) \mathrm{P} & \text { Phosphatidic acid } \\ \text { Phosphatidylethanolamine } & \text { Phosphatidylserine } \\ \text { Phosphatidylcholine } & \text { Blank }\end{array}$

His-Atg5 His-Atg16L1(1-265)

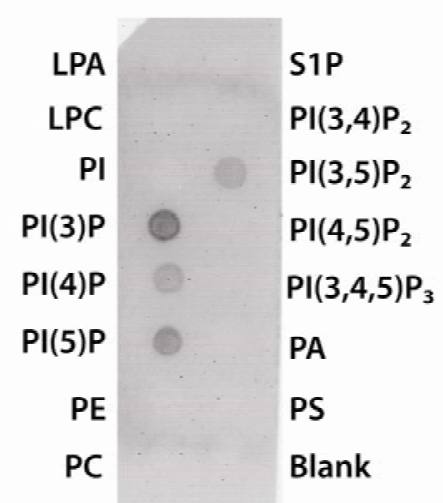

Figure 3.40: PIP strip protein lipid overlay assay using the Atg5 Atg16L1(1-70) and Atg5 Atg16L1(1-265) complexes. The PIP strips (Echelon) contain all naturally occurring phosphoinositides. Binding of the mammalian Atg5 Atg16L1 complexes at a concentration of $5 \mu \mathrm{g} / \mathrm{mL}$ to PIPs was detected with a murine monoclonal anti-His-tag antibody (Dianova).

The Atg5 Atg16L1(1-265) complex demonstrated a higher affinity for PIPs than the Atg5 Atg16L1(1-70) complex (Fig. 3.40). The difference could be due to the dimerization of the Atg5 Atg16L1(1-265) complex, which contains the complete Atg16L1 coiled coil domain. To further probe whether dimerization of the Atg5 Atg16L1 complex increases binding affinity to PIPs, PIP arrays which are quantitative protein lipid overlay assays were used. Here Atg5 Atg16L1 complexes which contained different lengths of the Atg16L1 coiled coil domain were tested.

The complexes showed interactions with phosphatidylinositol-monophosphates. In Figure 3.41, it can be seen that the minimal Atg5 Atg16L1(1-70) complex bound the weakest, requiring at least $50 \mathrm{pmol} \mathrm{PIP} / \mathrm{spot}$. The murine Atg5 Atg16L1(1-106) and Atg5 Atg16L1(1-113) complexes, comprising parts of the coiled coil domain, exhibited binding affinities similar to each other and bound stronger than the minimal complex (Fig. 3.41). For the murine Atg5 Atg16L1(1-265) complex with the full coiled coil domain, only $12.5 \mathrm{pmol} \mathrm{PIP/spot} \mathrm{were} \mathrm{needed} \mathrm{to} \mathrm{observe} \mathrm{an} \mathrm{interaction} \mathrm{(Figure} \mathrm{3.41).} \mathrm{The}$ presence of the full coiled coil domain of Atg16L1 increased the affinity roughly four-fold compared to the minimal complex (Figure 3.41). These data show that the coiled coil domain enhances PIP binding of the mammalian Atg5 Atg16L1 complex. 


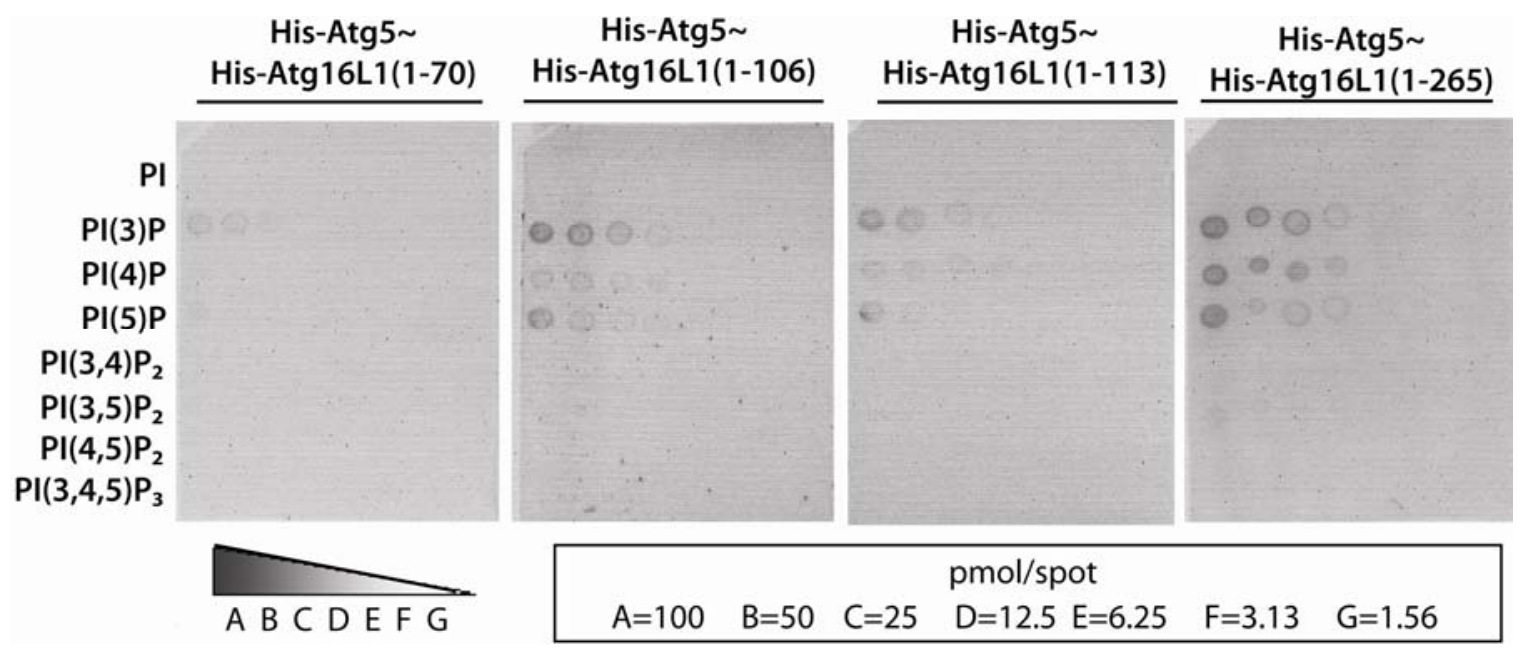

Figure 3.41: PIP array protein lipid overlay assay with mammalian Atg5 Atg16L1 complexes containing varying lengths of the coiled coil domain of Atg16L1. Protein lipid overlay assay with an assortment of PIPs (PIP array, Echelon) in decreasing amounts from left to right. Interaction of the mammalian His-Atg5 His-Atg16L1 complexes $(5 \mu \mathrm{g} / \mathrm{mL})$ with PIPs was shown via His-tag antibody detection.

\subsubsection{Phosphoinositide binding of the yeast Atg5 Atg16 complex}

In order to elucidate whether PIP binding of Atg5 Atg16L1 is evolutionarily conserved, the Saccharomyces cerevisiae Atg5 Atg16 complex was also tested for PIP interaction. It was attempted to purify the full-length Atg5 Atg16(1-150) complex by our technician, Michaela Hellwig. However the complex was insoluble and could not be purified. Three yeast Atg5 Atg16 complexes were cloned which contained varying lengths of Atg16 (Figure 3.42 panel A). The synthetic genes encoding Saccharomyces cerevisiae Atg5 and Atg16 were codon optimized for expression in E. coli (Mr.Gene, Regensburg). Atg5 and Atg16 were co-expressed using the pETDuet-1 plasmid. Atg5 was cloned into the first multiple cloning site as a His-tag protein and Atg16 was cloned into the second multiple cloning site without an affinity tag. Cloning of the Atg5 Atg16(1-46) and Atg5 Atg16(1-57) complexes was performed by our lab rotation student Sebastian Morales. Atg5 Atg16(1-67) cloning and purification was done by Michaela Hellwig. The purified complexes are shown in panel B of Figure 3.42. Atg16(1-46) and Atg16(1-57) comprise the Atg5-binding domain of Atg16 and were crystallized previously (Matsushita 2006). Atg16(1-67) comprises the first 10 residues of the coiled coil domain in addition to the Atg5-binding domain. Interaction of Atg16 with Atg5 was confirmed by its co-elution with His-Atg5 during IMAC purification (Fig. 3.43 panels A,C and E,F) followed by size exclusion chromatography with a HiLoad 16/60 Superdex 200 column (Fig. 3.43 panels B and D). Final yields of the yeast Atg5 Atg16(1-46) and Atg5 Atg16(1-57) complexes were $~ 5 \mathrm{mg}$ per liter culture. 


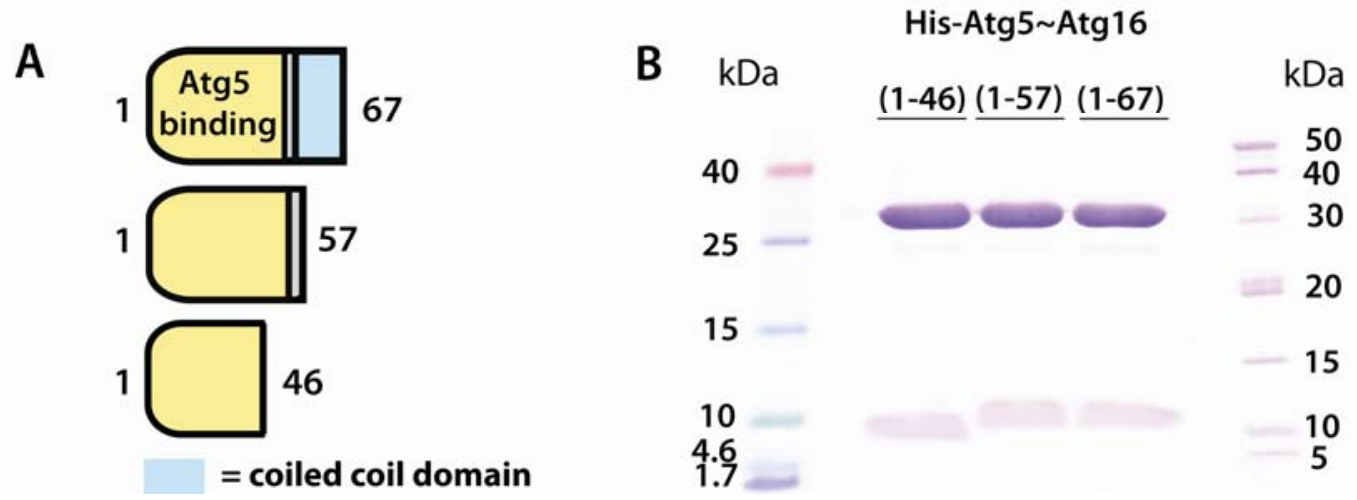

Figure 3.42: Yeast Atg5 Atg16L1 complexes used for lipid interaction studies. (A) Scheme of yeast Atg16 constructs co-purified with full-length Atg5. (B) 15\% SDS-PAGE gel of the three yeast Atg5 Atg16 complexes after gel filtration purification using a HiLoad 16/60 Superdex 200 column.

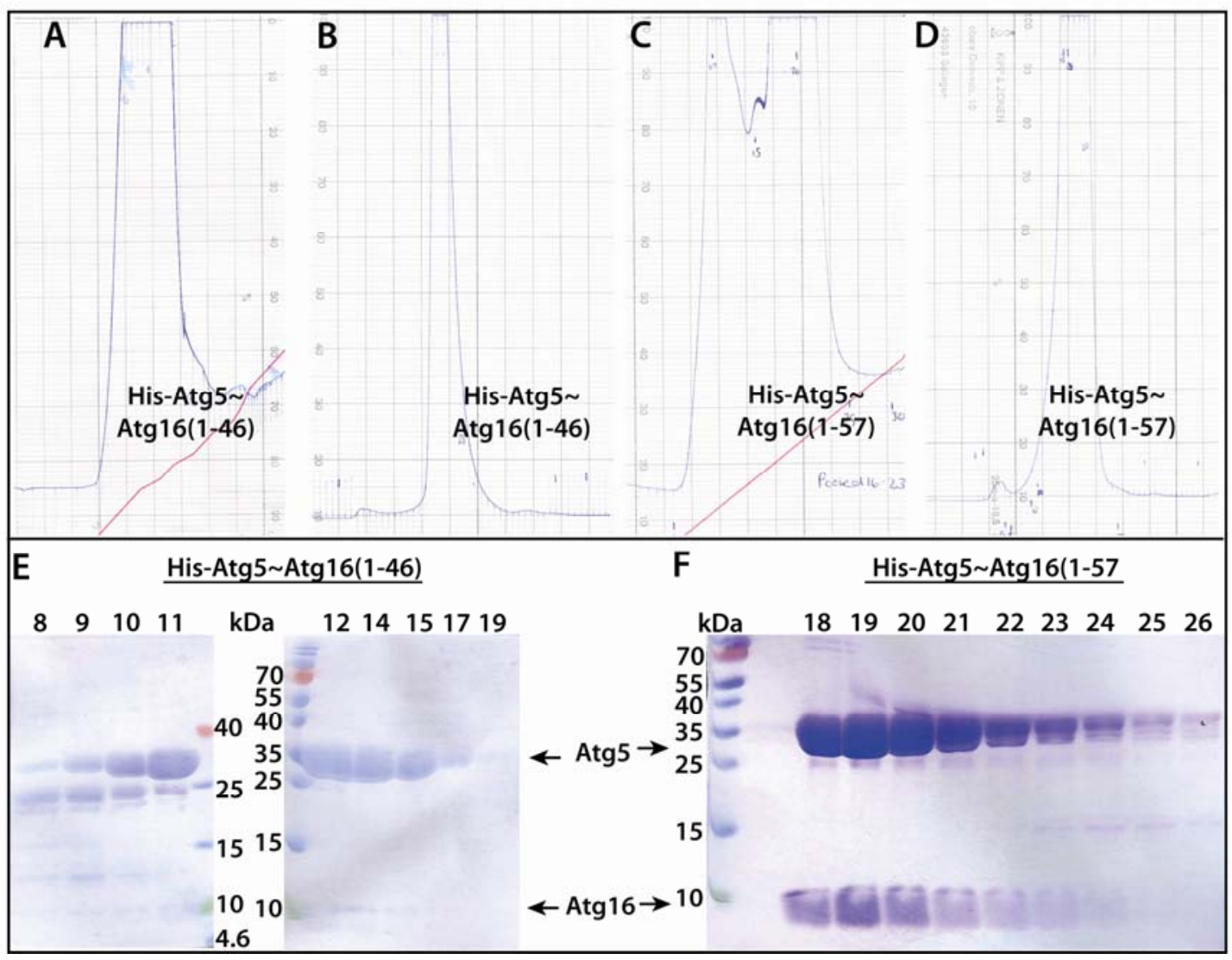

Figure 3.43: Purification of the yeast His-Atg5 Atg16(1-46) and His-Atg5 Atg16(1-57) complexes. Chromatograms of (A) Ni-sepharose column purification of His-Atg5 Atg16(1-46) followed by a (B) gel filtration run with a HiLoad 16/60 Superdex 200 column and (C) Ni-sepharose column purification of HisAtg5 Atg16(1-57) followed by a (D) gel filtration run with a HiLoad 16/60 Superdex 200 column. 15\% SDS-PAGE gels of Ni-sepharose purification of (E) His-Atg5 Atg16(1-46) and (F) His-Atg5 Atg16(1-57). Blue line $=U_{280}$ curve, red line $=\%$ elution buffer.

PIP strip protein lipid overlay assays were used to investigate whether the yeast Atg5 Atg16 complexes also bind phosphoinositides. All three yeast Atg5 Atg16 complexes bound phosphatidylinositol-monophosphates (Fig. 3.44), which shows that PIP binding of the Atg5 Atg16 complex is conserved from yeast to mammals. 


\begin{tabular}{|c|c|c|c|c|c|c|c|c|}
\hline & $(1-46)$ & & & $(1-57)$ & & & $(1-67)$ & \\
\hline LPA & & S1P & LPA & & S1P & LPA & & S1P \\
\hline LPC & & $\mathrm{PI}(3,4) \mathrm{P}_{2}$ & LPC & & $\mathrm{PI}(3,4) \mathrm{P}_{2}$ & LPC & & $\mathrm{PI}(3,4) \mathrm{P}_{2}$ \\
\hline PI & & $\mathrm{PI}(3,5) \mathrm{P}_{2}$ & PI & & $\mathrm{PI}(3,5) \mathrm{P}_{2}$ & PI & & $\mathrm{PI}(3,5) \mathrm{P}_{2}$ \\
\hline $\mathrm{PI}(3) \mathrm{P}$ & at. & $\mathrm{PI}(4,5) \mathrm{P}_{2}$ & $\mathrm{PI}(3) \mathrm{P}$ & & $\mathrm{PI}(4,5) \mathrm{P}_{2}$ & $\mathrm{PI}(3) \mathrm{P}$ & e & $\mathrm{PI}(4,5) \mathrm{P}_{2}$ \\
\hline $\mathrm{PI}(4) \mathrm{P}$ & t & $\mathrm{PI}(3,4,5) \mathrm{P}_{3}$ & $\mathrm{PI}(4) \mathrm{P}$ & & $\mathrm{PI}(3,4,5) \mathrm{P}_{3}$ & $\mathrm{PI}(4) \mathrm{P}$ & 항 & $\mathrm{PI}(3,4,5) \mathrm{P}_{3}$ \\
\hline $\mathrm{PI}(5) \mathrm{P}$ & $=$ & PA & $\mathrm{PI}(5) \mathrm{P}$ & 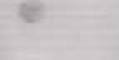 & PA & $\mathrm{PI}(5) \mathrm{P}$ & $\bullet$ & PA \\
\hline PE & & PS & PE & & PS & PE & & PS \\
\hline PC & & Blank & PC & & Blank & PC & & Blank \\
\hline
\end{tabular}

Figure 3.44: PIP strip protein lipid overlay assay using yeast His-Atg5 Atg16 complexes. Interaction of the yeast Atg5 Atg16 complexes at a concentration of $20 \mu \mathrm{g} / \mathrm{mL}$ was observed via His-tag antibody detection.

The His-Atg5 Atg16(1-67) complex, which included the first 10 residues of the coiled coil domain of Atg16, displayed the strongest binding of the three complexes (Fig. 3.44). Interestingly, unlike the mammalian complex it showed a higher affinity for PI(5)P than PI(3)P. These findings were also confirmed using the more quantitative PIP array protein lipid overlay assay. The Atg5 Atg16(1-67) complex showed a stronger interaction to the phosphatidylinositol-monophosphates compared to the minimal yeast complex, Atg5 Atg16(1-46), which lacks the coiled coil domain of Atg16 (Figure 3.45). These observations emphasize the enhancing effect of the Atg16 coiled coil domain which is consistent with our findings for the mammalian Atg5 Atg16L1 complex.

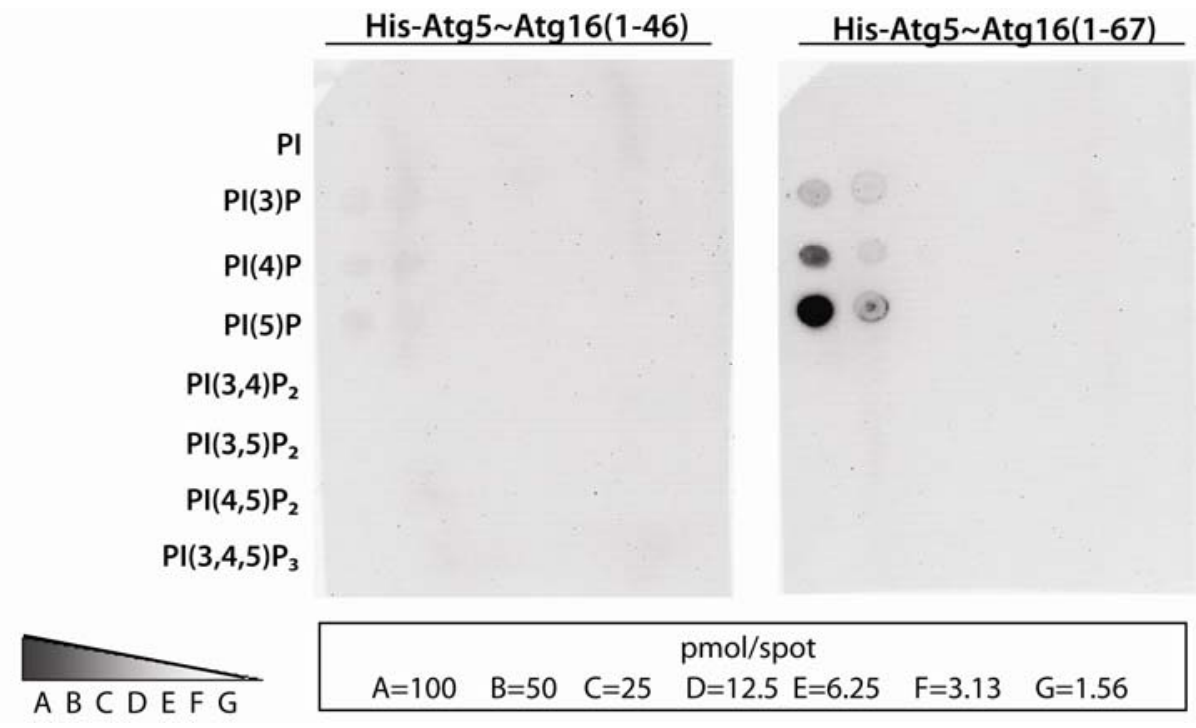

Figure 3.45: PIP array protein lipid overlay assay using the yeast His-Atg5 Atg16 complexes. Protein lipid overlay assay (PIP array, Echelon) containing an assortment of phosphoinositides in decreasing amounts. Interaction of the yeast Atg5 Atg16 complexes $(20 \mu \mathrm{g} / \mathrm{mL})$ with PIPs was observed via His-tag antibody detection. 


\subsubsection{Dissection of the interaction of Atg5 Atg16 with phosphoinositides}

While the coiled coil domain of Atg16 enhances phosphoinositide binding for both the mammalian and yeast complexes, the minimal Atg5-binding domain of Atg16 was sufficient to observe an interaction. The yeast Atg5 Atg16(1-57) structure, however, does not resemble any known PIP binding domain and the N-terminal region of Atg16 is missing in the yeast Atg5 Atg16 structure. Furthermore, the first 22 residues of Atg16 are disordered in the yeast $\operatorname{Atg} 5 \sim \operatorname{Atg} 16(1-46)$ structure. In the yeast $\operatorname{Atg} 5 \sim \operatorname{Atg} 16(1-57)$ structure, the first 21 residues of Atg15 are not included in the model (Matsushita 2007).

To gain further insight into the structure of the missing N-terminus of Atg16, secondary structure prediction was done with the JPRED3 server (Cole 2008). Both yeast Atg16 (Fig. 3.46 panel A) and murine Atg16L1 (Fig. 3.46 panel B) are predicted to have an additional $\alpha$-helix at their N-termini. As shown in the boxes on Figure 3.46 panels $\mathrm{A}$ and $\mathrm{B}$, the N-termini of $A \operatorname{tg} 16$ and $\operatorname{Atg} 16 \mathrm{~L} 1$ are rich in basic residues and the first predicted $\alpha$-helix has a basic patch. The N-termini of yeast and mammalian Atg16 are only $67 \%$ similar according to alignment performed by T-COFFEE version 7.38 (Notredame 2000, Poirot 2003). It is of note that the N-terminus of Atg16 can vary between different species of fungi as well as higher eukaryotes like Xenopus tropicalis. This is shown in a multiple sequence alignment performed by T-COFFEE and shown in Figure 3.46 (Notredame 2000, Poirot 2003).

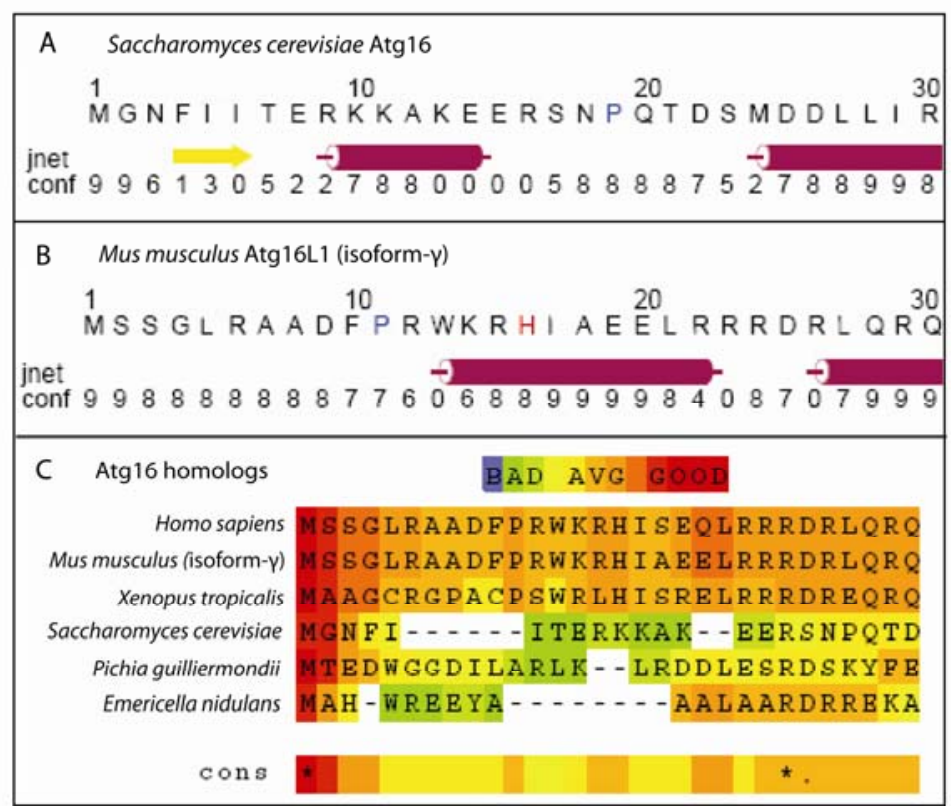

Figure 3.46: Secondary structure prediction and multiple alignments of Atg16 from yeast to murine. The JPRED3 server (http://www.compbio.dundee.ac.uk/www-jpred/) was used to predict the secondary structure of (A) yeast Atg16 and (B) murine Atg16L1. (C) The T-COFFEE server was used to align homologues of Atg16 from fungal species up to higher eukaryotes (http://www.igs.cnrs-mrs.fr/Tcoffee). 
To test whether these N-terminal basic residues of Atg16L1 might be important for binding, the His-Atg5 His-Atg16L1(24-168) complex, where the N-terminus of Atg16L1 is deleted, was examined for PIP binding. As seen in Figure 3.47 panel A, only a very weak signal can be detected for PI(3)P. The reduction in PIP binding could be due to the absence of these N-terminal basic residues of Atg16L1, or alternatively the reduction in PIP binding could be due to a disruption in complex formation between Atg5 and Atg16L1. Indeed, the deletion of the N-terminal 23 residues of Atg16L1 greatly diminished the amount of Atg5 bound during co-purification of the complex (Fig. 3.47 panel B).

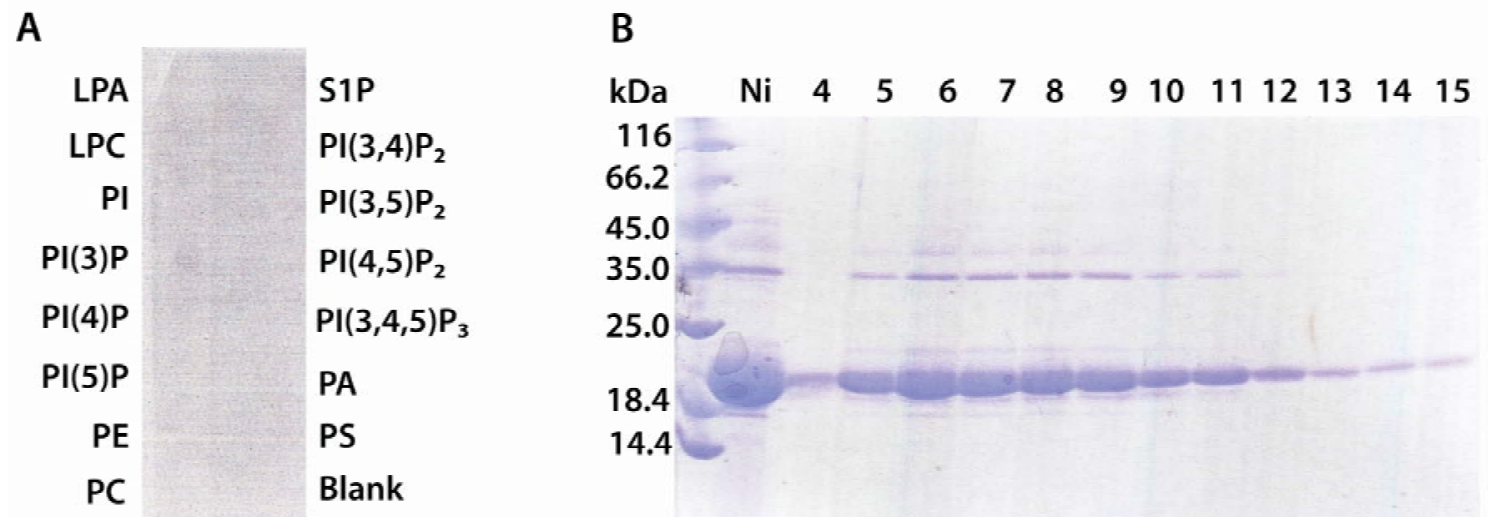

Figure 3.47: PIP strip protein lipid overlay assay using the His-Atg5 His-Atg16L1(24-168) complex. (A) Protein lipid overlay assay (PIP strip, Echelon) with the His-Atg5 His-Atg16L1(24-168) complex at a concentration of $5 \mu \mathrm{g} / \mathrm{mL}$. Binding was detected by a monoclonal His-tag antibody (Echelon). (B) $15 \%$ SDS-PAGE gel of the Atg5 Atg16L1(24-2168) complex after gel filtration purification using a HiLoad 16/60 Superdex 200 column.

It was then investigated whether the first 25 residues of murine Atg16L1 alone would be sufficient for PIP binding using a synthesized peptide (Biosyntan) with a StrepIItag at the C-terminal end. To optimize the concentration of Atg16L1 peptide required for detection, a dot blot assay was used (Figure 3.48. panel A). However, no binding of Atg16L1(1-25)-StrepII was observed in the PIP strip protein lipid overlay assay (Figure 3.48 panel B). 


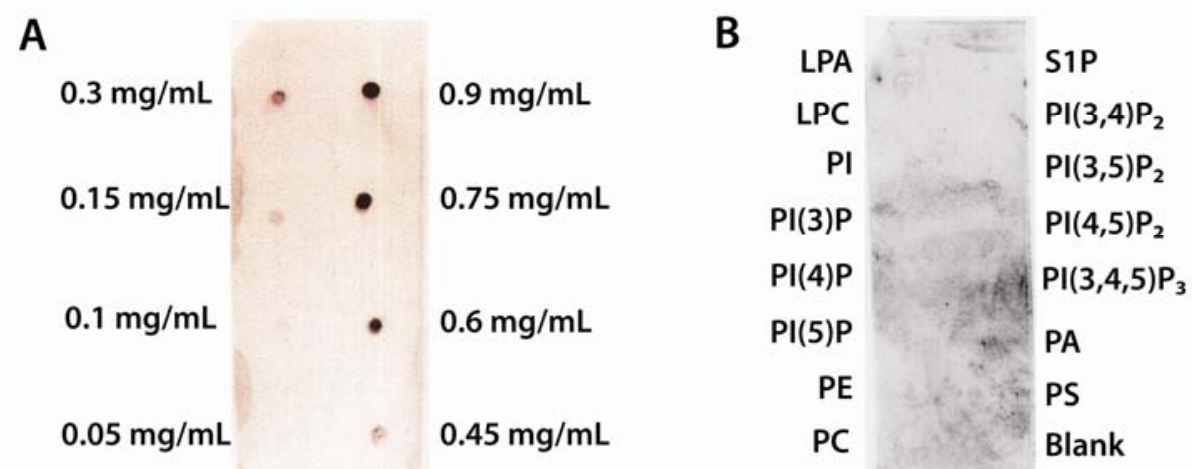

Figure 3.48. Dot blot assay and protein lipid overlay assay the using Atg16L1(1-25)-Strepll peptide. (A) Dot blot assay with varying concentrations of the Atg16L1(1-25)-Strepll peptide spotted onto the membrane to determine which concentration was sufficient for detection with murine antiStrep-tag II antibody (IBA GmbH). (B) PIP strip protein lipid overlay assay, where anti-Strepll-tag antibody was used for detection of peptide binding.

These data show that the basic N-terminus of Atg16L1 on its own is insufficient for binding to PIPs and that the association of Atg5 and Atg16L1 is essential for an interaction of Atg5 Atg16L1 with phosphoinositides.

Based on these results, a series of Atg16L1(1-265) mutants were made to convert basic residues in the N-terminus to nonpolar alanines and test how binding was affected (Fig. 3.49). Residues K14, R15, and R22 were all mutated separately. A double mutant comprising K14A and R15A was prepared. Additionally, a triple mutant containing all three residues (K14A, R15A, R22A) was also constructed.

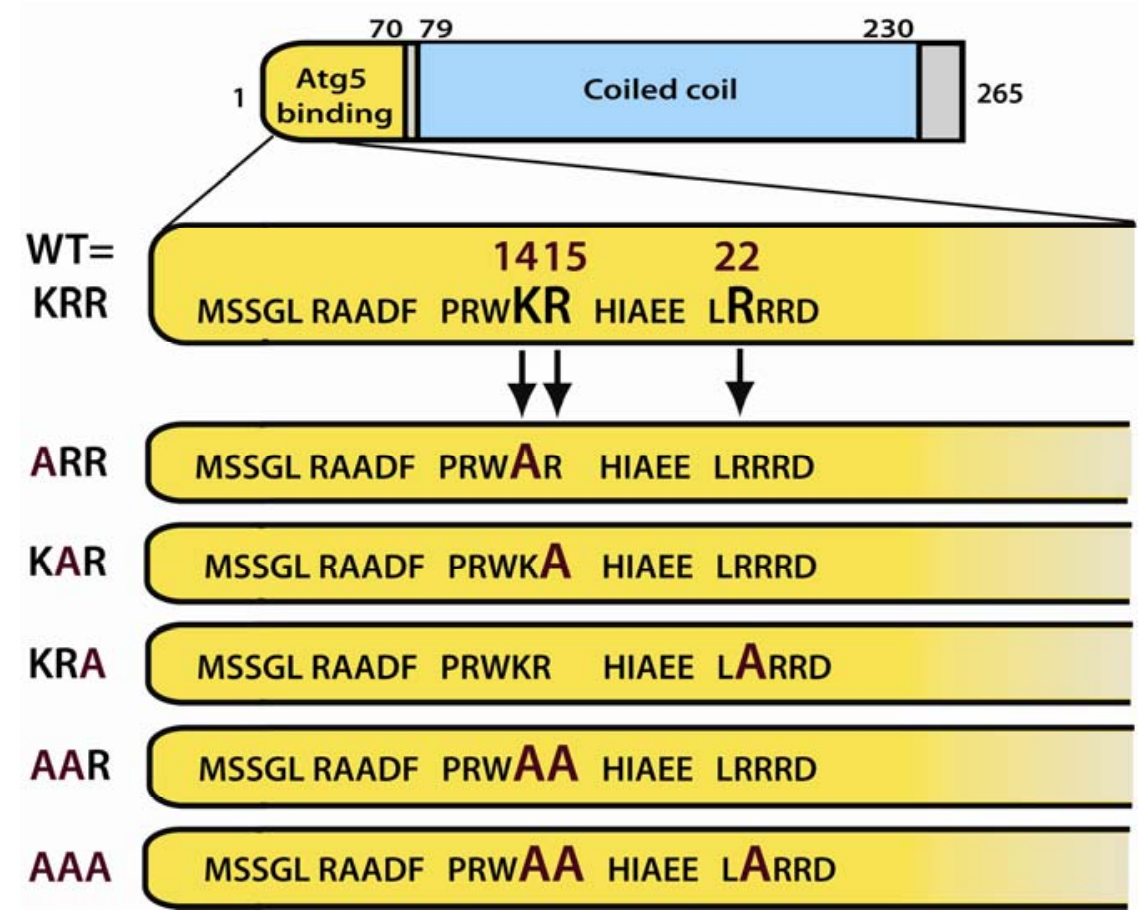

Figure 3.49: Scheme of mutations of murine Atg16L1 $\mathrm{N}$-terminal basic residues. Site-directed mutagenesis was performed to mutate K14, R15, and R22 to nonpolar alanines. A double mutant construct comprising K14A and R15A and the triple mutant construct were prepared. 
As shown in Figure 3.50, the mutation of these Atg16L1 basic residues to nonpolar alanines impaired PIP binding of Atg5 Atg16L1(1-265). Binding was almost entirely abolished for the ARR (K14A) mutant and was slightly reduced for the KRA (R22A) mutant. The KAR (R15A) mutant showed similar strength in binding to the wild-type. The double and triple mutants do not bind to phosphoinositides.

An important aspect to take into account for the PIP strip protein lipid overlay assay is the amount of time that the lipids on the membranes are being incubated in aqueous buffer (Narayan 2006). The solubility of $\mathrm{PI}(3,4,5) \mathrm{P}_{3}$ in aqueous buffer is greater than that of the $\mathrm{PIP}_{2} \mathrm{~S}$ due to the polarity of the phosphate groups. In turn, the $\mathrm{PIP}_{2} \mathrm{~S}$ are more water-soluble than PI(3)P, PI(4)P, and PI(5)P. To reduce the effect of $\mathrm{PIP}_{2} \mathrm{~S}$ and $\mathrm{PIP}_{3}$ being washed off the membrane, the length of incubation times was reduced by using a His-tag antibody conjugated with horseradish peroxidase (HRP) to eliminate the incubation step with the secondary antibody and associated washing steps.

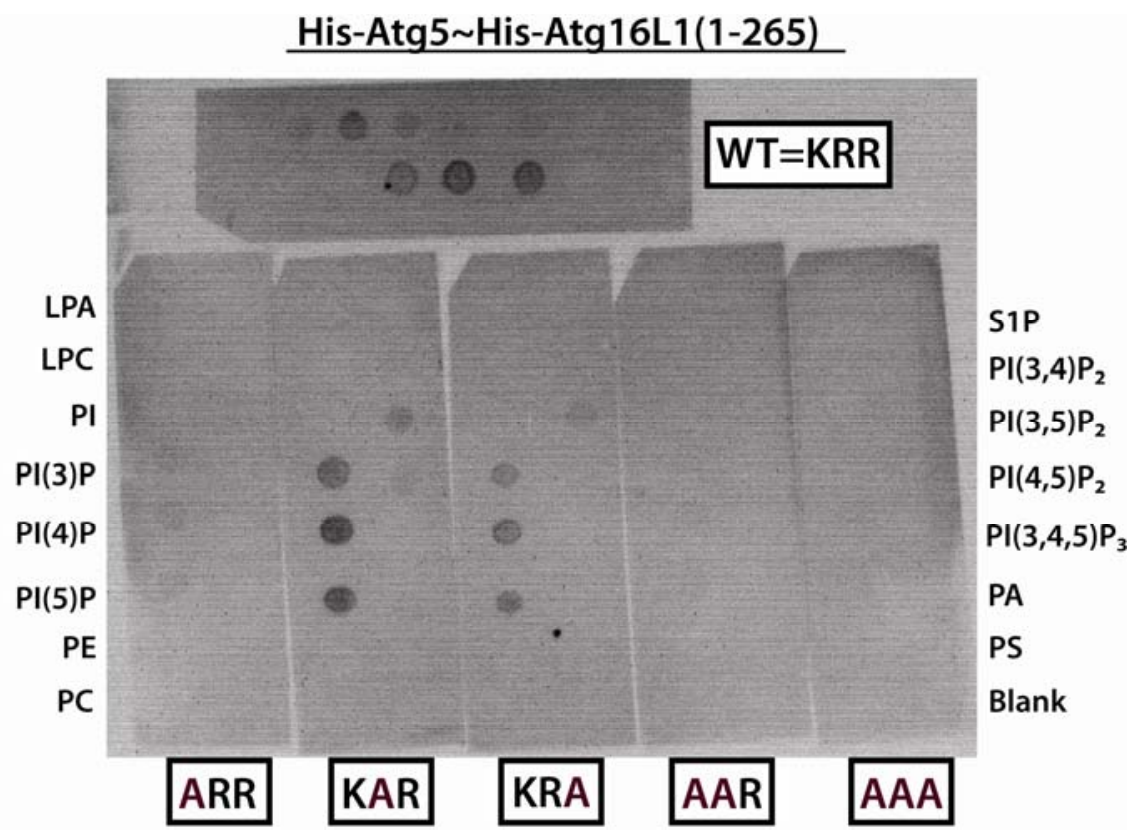

Figure 3.50: PIP strip protein lipid overlay assay using mammalian His-Atg5 His-Atg16L1(1-265) wild-type and $\mathbf{N}$-terminal mutant complexes. Binding of the mammalian His-Atg5 His-Atg16L1 (1-265) wild type and mutant complexes $(5 \mu \mathrm{g} / \mathrm{mL})$ was detected via a His-tag antibody. ARR, KAR, and KRA are the single mutants $K 14 A, R 15 A$, and R22A, respectively. AAR denotes the K14AR15A double mutant. AAA denotes the triple mutant.

Indeed, decreasing the incubation time of the membrane in aqueous buffer increased the overall signal of Atg5 Atg16L1(1-265) complex binding (Figure 3.51). This improved protocol also led to the detection of $\mathrm{PI}(3,5) \mathrm{P}_{2}$ binding of mammalian Atg5 Atg16L1(1-265). Even with an increase of signal strength, a drastic reduction of PIP binding by the triple mutant compared to the wild-type was observed. The single 
mutant ARR (K14A) also exhibited a reduction in PIP binding to a similar extent as seen for the triple mutant.

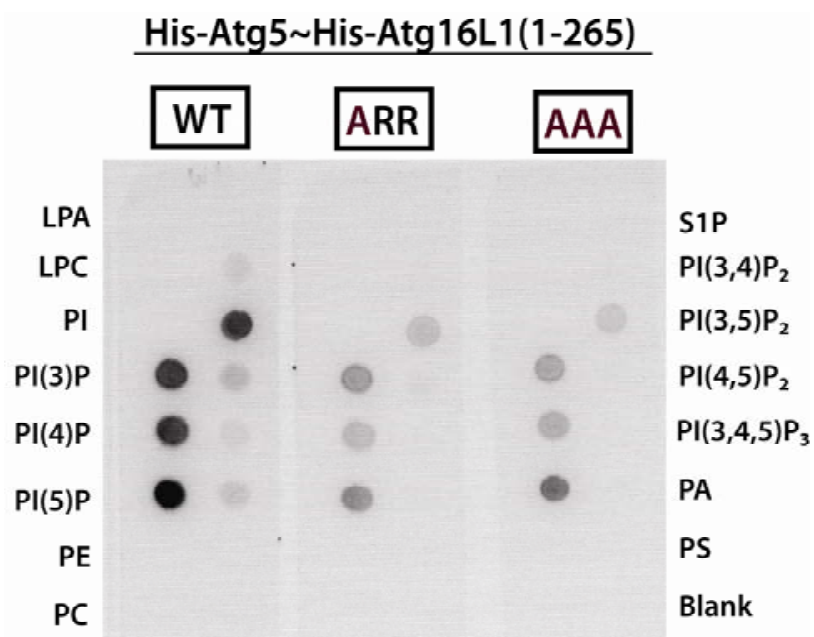

Figure 3.51: PIP strip protein lipid overlay assay using mammalian His-Atg5 His-Atg16L1(1-265) wild type and mutant complexes. The total length of incubation of the PIP strips (Echelon) in aqueous buffer was reduced by detection with a His-tag antibody directly coupled to HRP. The protein complexes were used at a concentration of $5 \mu \mathrm{g} / \mathrm{mL}$.

To investigate if basic residues essential for PIP binding could also be identified in yeast Atg16, the Atg5 Atg16(1-67) complex was used to create a series of single and double mutants as well as a triple mutant for basic residues in the N-terminus of Atg 16. Site-directed mutagenesis as well as purification of these complexes was done by our technician Michaela Hellwig. A scheme of the generated mutants is shown in Figure 3.52.

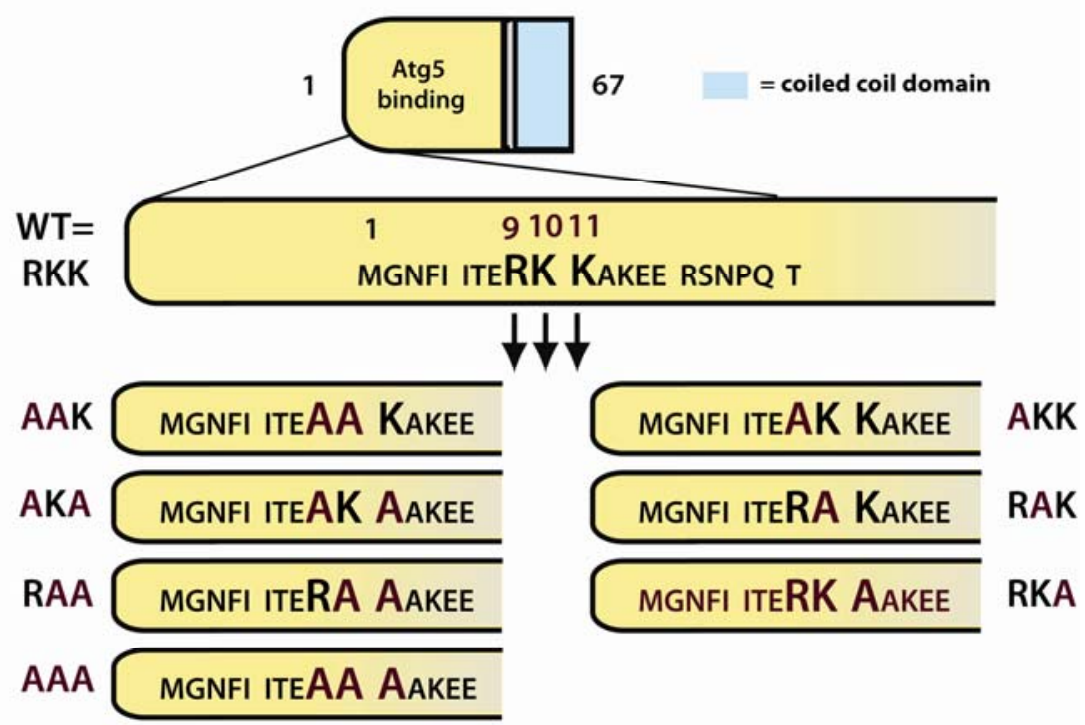

Figure 3.52: Scheme of single, double, and triple mutations of yeast Atg16 N-terminal basic residues to alanines as part of the Atg5 Atg16(1-67) complex. The wild-type is denoted as RKK. AKK, RAK, and RKA are the single mutants R9A, K10A, and K11A, respectively. AAK, AKA, and RAA denote the double mutants R9AK10A, R9AK11A, and K10AK11A, and AAA denotes the triple mutant. Site-directed mutagenesis of the yeast Atg5 Atg16(1-67) complex and purification of the mutants was performed by our technician, Michaela Hellwig. 
Protein lipid overlay assays were conducted on the series of yeast Atg5 Atg16(167) wild-type and mutant complexes. All combinations of the double mutants as well as the triple mutant displayed almost complete abolishment of PIP binding (Fig. 3.53 second row). The single mutants RAK (K10A) and RKA (K11A) were also impaired in PIP binding (Fig. 3.53 first row). A striking phenotype was observed for the AKK (R9A) mutant. A markedly increased binding affinity to PIPs was observed in comparison to the wild-type complex (Fig. 3.53 first row).

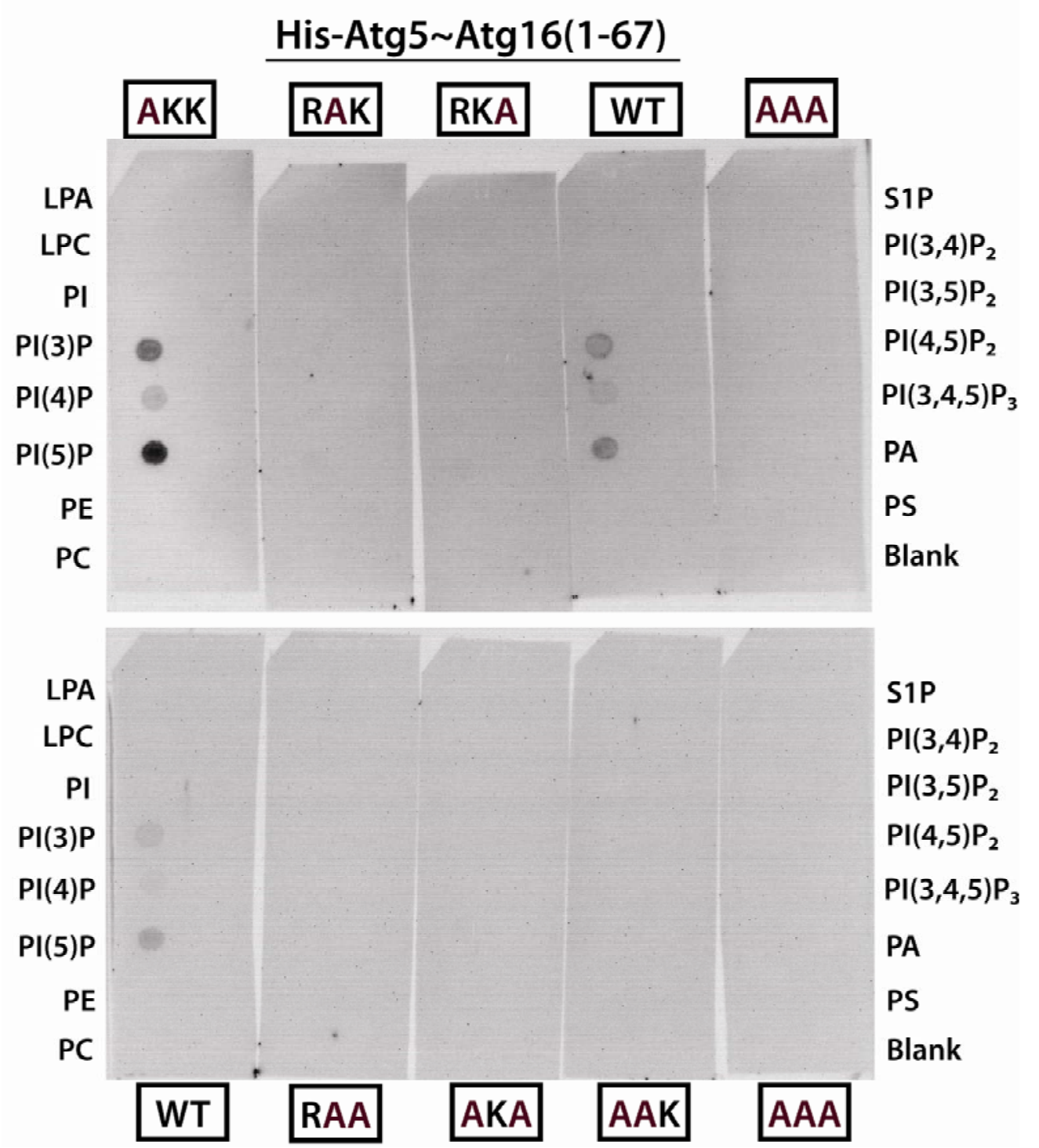

Figure 3.53: PIP strip protein lipid overlay assays of the yeast His-Atg5 Atg16(1-67) wild-type as well as the single, double, and triple mutant complexes. The interaction of the yeast Atg5 Atg16 (167) RKK (wild-type) $(20 \mu \mathrm{g} / \mathrm{mL})$ or mutant complexes with PIPs was observed via detection with a His-tag antibody coupled to HRP. 
Next it was investigated whether the increased signal of the AKK (R9A) mutant compared to the wild-type complex could be due to possible aggregation of the latter. However, it was shown by analytical gel filtration that neither the wild-type Atg5 Atg16(1-67) RKK complex nor the AKK (R9A) or AAA mutant complexes eluted in the void volume of the column which would indicate aggregation (Fig. 3.54). Using Dextran Blue (MW $2000 \mathrm{kDa}$ ), the void volume of the column was determined to be approximately $8 \mathrm{~mL}$.

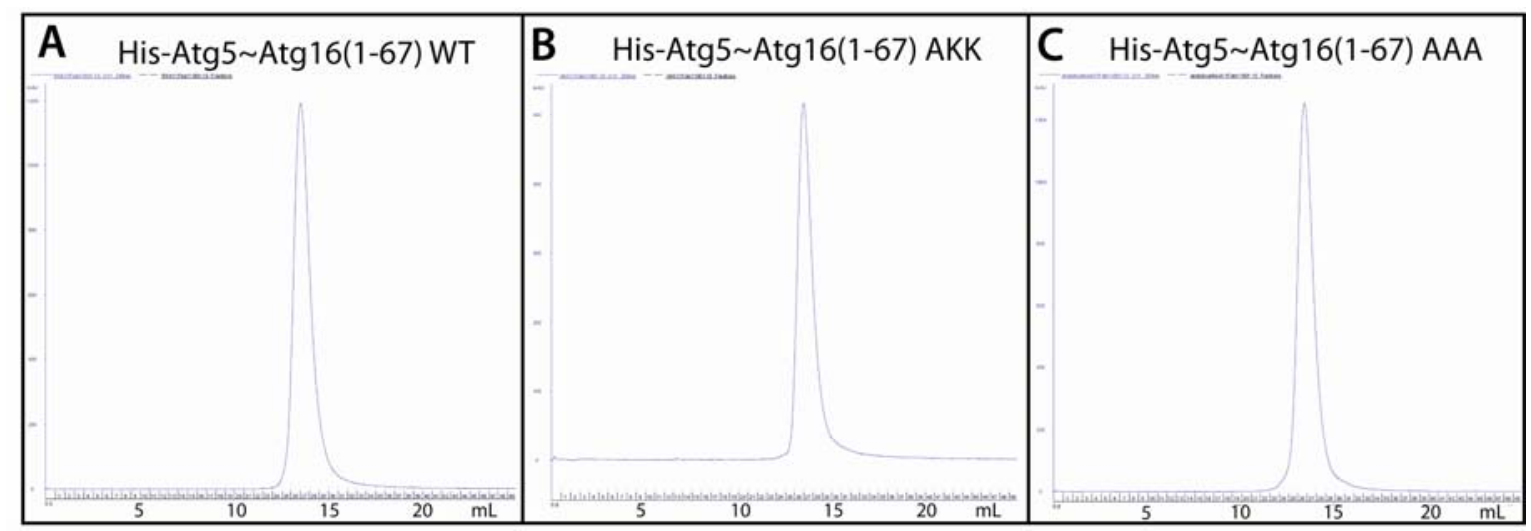

Fig. 3.54: Analytical gel filtration of yeast His-Atg5 Atg16(1-67) RKK (wild-type), AKK (R9A), and triple mutant AAA (R9A,K10A,K11A) complexes. The Atg5 Atg16(1-67) complexes (A) RKK (wildtype), (B) AKK (R9A), and (C) AAA (R9A,K10A,K11A) were diluted in the buffer used for protein lipid overlay assays and loaded onto a Superdex 200 10/300 GL column.

The increased affinity of the His-Atg5 Atg16(1-67) AKK (R9A) mutant for PIPs was also not due to an increased amount of protein used since their concentrations were carefully determined and the samples were stepwise diluted into the blocking buffer used for incubation with the PIP strips. As an additional control that equal amounts of proteins were used, samples were run on a 15\% SDS-PAGE gel (Fig. 3.55).

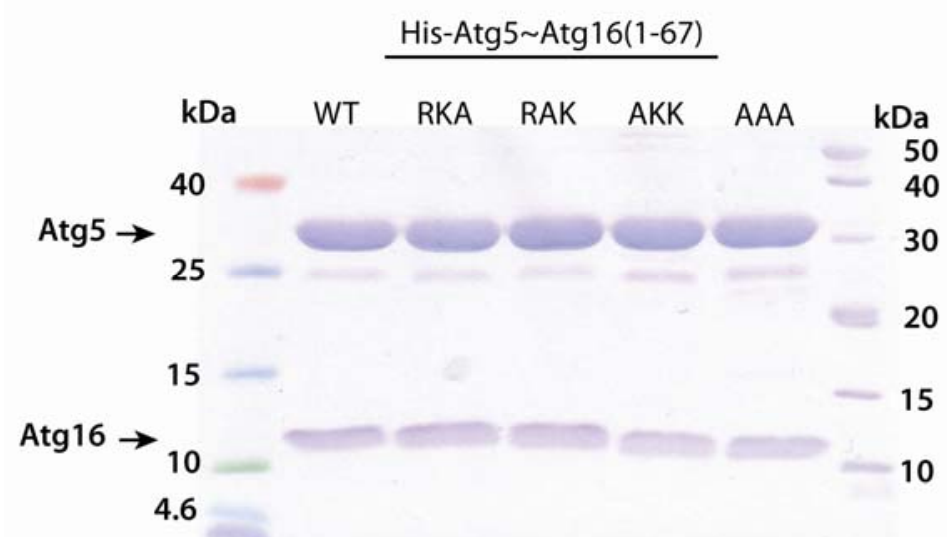

Fig. 3.55: 15\% SDS-PAGE gel of the Atg5 Atg16(1-67) wild-type and mutant complexes. The protein complexes were diluted to $1.0 \mathrm{mg} / \mathrm{mL}$ before being further diluted into blocking buffer with a final concentration of $20 \mu \mathrm{g} / \mathrm{mL}$. 


\subsubsection{Atg5 Atg16L1 binds phosphoinositide-containing liposomes}

Protein lipid overlay assays are a fast way to test for protein-lipid interactions. However, since the lipids are in a non-physiological setting immobilized on a flat surface and highly concentrated in a small area, a second method was employed to confirm binding. Flotation assays were utilized to investigate binding of the murine HisAtg5 His-Atg16L1(1-265) complex to phosphoinositides incorporated into liposomes. Since the exact lipid composition of autophagosomes is still unknown, the lipid composition of endosomes was used instead to mimic a physiological membrane which contains PI(3)P (Ohya 2009). As shown in Figure 3.56, more of the Atg5 Atg16L1(1265) complex floated to the top with the PI(3)P-containing liposomes compared to the liposomes that lacked PI(3)P.

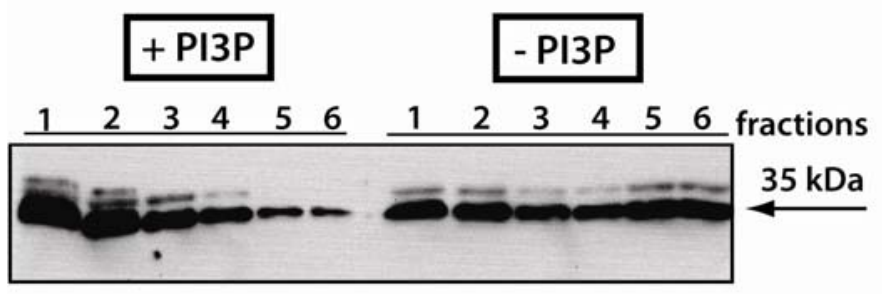

Figure 3.56: Flotation assay of the murine His-Atg5 His-Atg16L1(1-265) complex with liposomes either with or without PI(3)P. Small unilamellar vesicles contained $23 \% \mathrm{PE}, 2 \%$ Oregon Green-PE, 9\% SM, 9\% PS, 9\% PI, 43/48\% PC, and 5/0\% PI(3)P (Ohya 2009). Fraction 1 was collected where the liposomes floated up to the top of the ultracentrifuge tube. Fractions were then taken to the bottom of the tube where protein that did not bind to the liposomes would remain. The protein complex was detected by immunoblotting with an anti-His tag antibody.

His-Atg5 Atg16L1(1-265) co-flotation with liposomes was further enhanced when $\mathrm{PI}(4,5) \mathrm{P}_{2}$ was also incorporated into the liposomes (Fig. 3.57). This could be due to its higher net negative charge to which the basic patch on Atg16L1 could be attracted. However, $\mathrm{PI}(4,5) \mathrm{P}_{2}$ binding was not observed with the PIP strips.

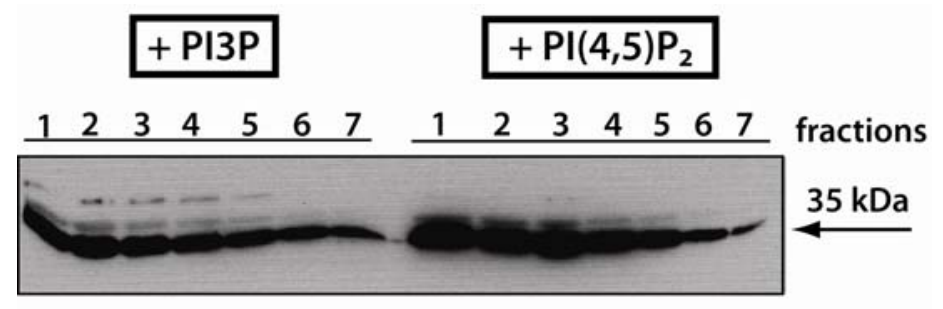

Figure 3.57: Flotation assay of the His-Atg5 His-Atg16L1(1-265) complex with liposomes containing $\mathbf{P I}(\mathbf{3}) \mathbf{P}$ or $\mathbf{P I}(\mathbf{4}, \mathbf{5}) \mathbf{P}_{2}$. Small unilamellar liposomes contained $23 \% \mathrm{PE}$, 2\% Oregon Green-PE, $10 \% \mathrm{SM}, 5 \% \mathrm{PS}, 5 \% \mathrm{PI}, 50 \% \mathrm{PC}$, and $5 \% \mathrm{PI}(3) \mathrm{P} / \mathrm{PI}(4,5) \mathrm{P}_{2}$. Fractions were collected from the top of the ultracentrifuge tube (fraction 1) to the bottom (fraction 7). The presence of the protein complex was detected by immunoblotting with an anti-His tag antibody. 
Even when using a non-physiological lipid composition where PI(3)P was the only negatively charged phospholipid, the His-Atg5 His-Atg16L1(1-265) complex co-migrated with liposomes (Fig. 3.58). Furthermore, this co-migration was greatly reduced for the His-Atg5 His-Atg16L1(1-265) AAR (K14A,R15A) double mutant in comparison to the wild-type complex. This observation gives additional evidence to the importance of these basic residues for PIP binding.

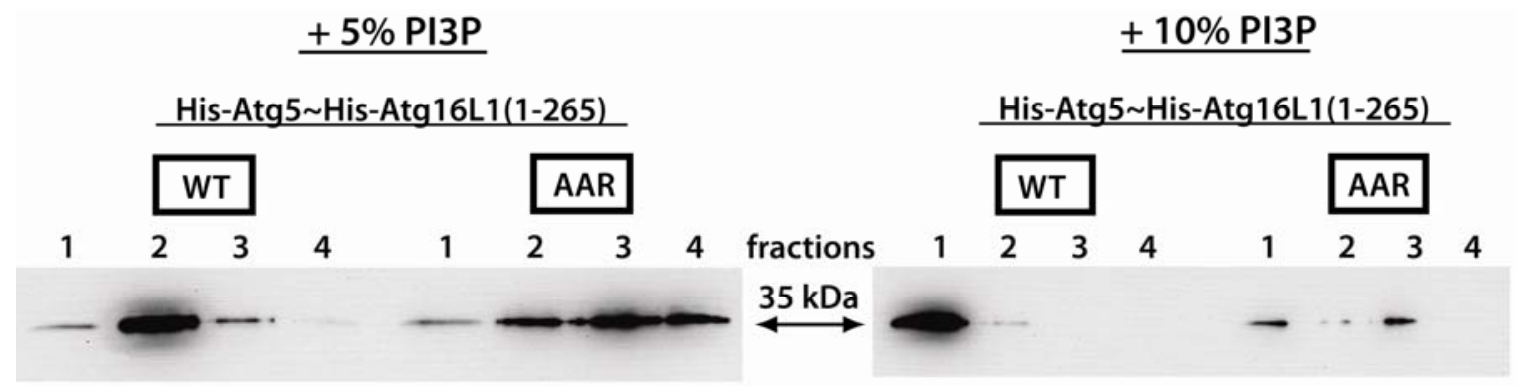

Figure 3.58: Flotation assay of PI(3)P-containing liposomes with the His-Atg5 His-Atg16L1(1-265) KRR (wild-type) and AAR double mutant (K14A,R15A) complexes. Small unilamellar liposomes contained $23 \% \mathrm{PE}$, $2 \%$ Texas Red-PE, 70/65\% PC, and 5/10\% PI(3)P. Fractions were collected from the top of the ultracentrifuge tube (fraction 1) to the bottom (fraction 4). The presence of the protein complex was detected by immunoblotting with an anti-His tag antibody.

\subsubsection{Limited proteolysis of Atg5 Atg16L1(1-106) complex with IP(1,3)}

Limited proteolysis experiments with Atg5 Atg16L1(1-106) were carried out either in the presence or absence of $\operatorname{IP}(1,3)_{2}$. If $\operatorname{IP}(1,3)_{2}$ binding induces a conformational change in the protein complex, i.e. the predicted N-terminal $\alpha$-helix becomes ordered upon IP $(1,3)_{2}$ binding, a different digestion pattern might be observed. Twelve different proteases from the Proti-Ace kit (Hampton Research) were tested. When the reactions were done at $37{ }^{\circ} \mathrm{C}$, the samples were completely digested. Therefore, the reaction temperature was lowered to room temperature. In digestion experiments done with $\alpha$ chymotrypsin and clostripain, a band from the Atg5 Atg16L1(1-106) complex in the presence of $\operatorname{IP}(1,3)_{2}$ appeared to be slightly more stable compared to when the complex was digested alone (Figure 3.59). But there are no clear differences between the digestions performed with or without $\operatorname{IP}(1,3)_{2}$.

However, since the identification of shorter, stable fragments could be useful in subsequent crystallization experiments, the reactions were up-scaled and bands were immobilized on PVDF membrane and sent for N-terminal sequencing. N-terminal sequencing results revealed that the first four amino acids of the Atg16L1 fragment 
cleaved by $\alpha$-chymotrypsin were Y-A-A-D. This does not match the sequence of Atg16L1. However, ${ }^{6} \mathrm{R}-\mathrm{A}-\mathrm{A}-\mathrm{D}^{9}$ occurs at the N-terminus of Atg16L1, directly after a leucine residue, after which $\alpha$-chymotrypsin can cleave. This fragment corresponds to 12 $\mathrm{kDa}$ which fits with the size of the fragment seen in the gel (Figure 3.59 panel A). This cleavage site would precede the predicted N-terminal $\alpha$-helix.

Clostripain, which is also referred to as endoproteinase Arg-C, cleaves the peptide bonds of arginine residues on the carboxyl side. The first four residues determined by Nterminal sequencing were $\mathrm{X}-\mathrm{S}-\mathrm{H}-\mathrm{M}$, where $\mathrm{X}$ could not be assigned. These residues would best correspond to G-S-H-M which occurs after an arginine and would produce a $12.9 \mathrm{kDa}$ fragment matching the size of the fragment seen on the Schägger Jagow gel in Figure 3.59 panel B. These residues are not within the sequence of Atg16L1. Instead they belong to the thrombin cleavage site after the His-tag as part of the pET-28a plasmid. These results indicate that the N-terminal region of Atg16 is ordered.

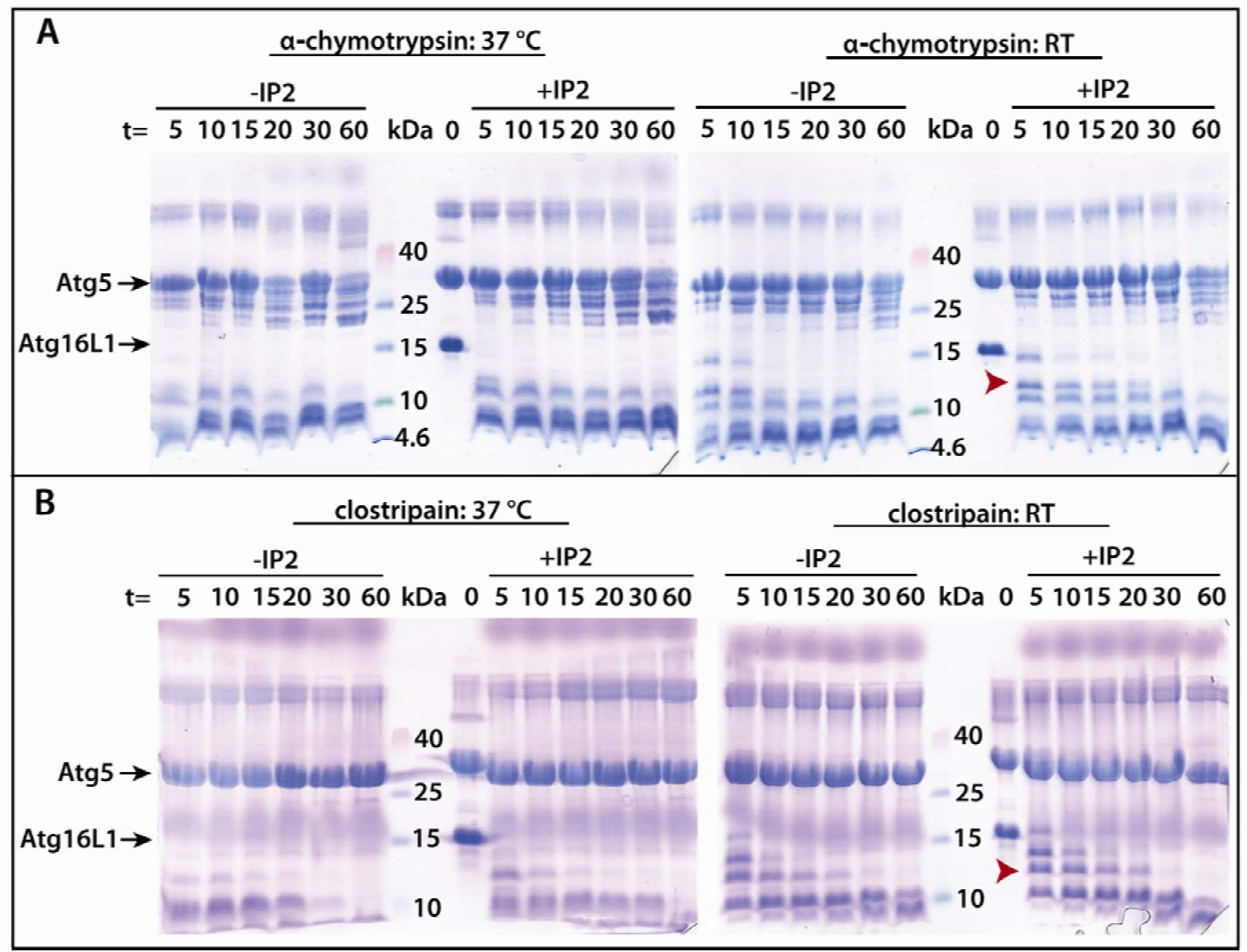

Figure 3.59: Digestions of the murine Atg5 Atg16L1(1-106) complex with or without $\operatorname{IP}(1,3)_{2}$. Limited proteolysis time course $(\mathrm{t}=\mathrm{min})$ with a-chymotrypsin and clostripain of the murine Atg5 Atg16L1(1-106) complex in either the presence or absence of $\operatorname{IP}(1,3)_{2}$. Schägger Jagow gels of digestions with (A) a-chymotrypsin and (B) clostripain are shown.. The red arrowheads indicate the bands that were sent for $\mathrm{N}$-terminal sequencing. 


\subsubsection{Crystallization of mammalian Atg5 Atg16L1 complexes with inositol diphosphate}

\section{Mammalian Atg5 Atg16L1(1-70)}

To elucidate the interaction between Atg5 Atg16L1 and PIPs, crystallization screens in 96-well Greiner or MRC sitting drop plates of the mammalian Atg5 Atg1616L1(1-70) complex and the inositol headgroup moiety from PI(3)P, D-myoInositol-1,3-disphosphate (sodium salt, Cayman) [IP $\left.(1,3)_{2}\right]$, were set up with the following commercial screens from Qiagen: Classics, ClassicsII, PEGs, PEGsII, Protein Complex, JCSG+, and the Cryos suite. A Cartesian dispensing robot (Genomic Solutions) was used for pipetting of the drops. Screens were set up with a wide array of combinations of the variable factors: temperature $\left(20{ }^{\circ} \mathrm{C}\right.$ and $\left.4{ }^{\circ} \mathrm{C}\right)$, different protein and $\operatorname{IP}(1,3)_{2}$ concentrations, as well as changes in the molar ratio between $\operatorname{IP}(1,3)_{2}$ and the Atg5 Atg16L1(1-70) complex. The Atg5 Atg16L1(1-70) complex + IP(1,3) $)_{2}$ crystals typically grew within one week in PEG-containing solutions. These conditions are summarized in Table 3.10. Different crystal morphologies were observed, including cubelike and rod-like shapes and thin plates (Fig. 3.60).

Table 3.10: Initial screening conditions of mammalian Atg5 Atg16L1(1-70) with $\mathrm{IP}(1,3)_{2}$

\begin{tabular}{|c|c|c|c|c|c|c|}
\hline & Buffer & Precipitant (w/v)/ Salt & {$\left[\mathrm{IP}(\mathbf{1}, \mathbf{3})_{2}\right]$} & [Protein] & $\begin{array}{c}\text { Molar } \\
\text { Ratio } \\
\end{array}$ & Temp. \\
\hline $\mathbf{1 A}$ & $0.1 \mathrm{M}$ sodium acetate $\mathrm{pH} 5.5$ & $\begin{array}{l}10 \% \text { PEG } 2000 \mathrm{MME}, \\
0.2 \mathrm{M} \text { ammonium sulfate }\end{array}$ & $1.04 \mathrm{mM}$ & $0.87 \mathrm{mM}$ & $1.2: 1$ & $4^{\circ} \mathrm{C}$ \\
\hline 1B & $0.1 \mathrm{M}$ sodium acetate $\mathrm{pH} 5.5$ & $\begin{array}{l}10 \% \text { PEG } 2000 \mathrm{MME} \\
0.2 \mathrm{M} \text { ammonium sulfate }\end{array}$ & $1.36 \mathrm{mM}$ & $0.68 \mathrm{mM}$ & $2: 1$ & $4^{\circ} \mathrm{C}$ \\
\hline $\mathbf{1 C}$ & $0.1 \mathrm{M}$ sodium acetate $\mathrm{pH} 5.5$ & $\begin{array}{c}10 \% \text { PEG } 2000 \mathrm{MM}, \\
0.2 \mathrm{M} \text { ammonium sulfate E}\end{array}$ & $1.62 \mathrm{mM}$ & 0.54 & $3: 1$ & $4^{\circ} \mathrm{C}$ \\
\hline $\mathbf{2 A}$ & $0.1 \mathrm{M}$ HEPES pH 7 & $\begin{array}{c}10 \% \text { PEG } 4000,10 \%(\mathrm{v} / \mathrm{v}) \\
\text { isopropanol }\end{array}$ & 1.04 & $0.8^{\prime}-x-3$ & $1.2: 1$ & $4^{\circ} \mathrm{C}$ \\
\hline 2B & $0.1 \mathrm{M}$ HEPES pH 7 & $\begin{array}{c}\text { 10\% PEG 4000, } 10 \%(\mathrm{v} / \mathrm{v}) \\
\text { isopropanol }\end{array}$ & $1.36 \mathrm{mM}$ & 0.68 & $2: 1$ & $4^{\circ} \mathrm{C}$ \\
\hline $2 \mathrm{C}$ & $0.1 \mathrm{M}$ HEPES $\mathrm{pH} 7$ & $\begin{array}{l}\text { 10\% PEG 4000, } 10 \%(\mathrm{v} / \mathrm{v}) \\
\text { isopropanol }\end{array}$ & $1.62 \mathrm{mM}$ & 0.5 & $3: 1$ & $4^{\circ} \mathrm{C}$ \\
\hline 3 & 0.1 Bis-Tris pH 5.5 & $2 \mathrm{M}$ ammonium sulfate & $1.36 \mathrm{mM}$ & $0.68 \mathrm{mM}$ & $2: 1$ & $4^{\circ} \mathrm{C}$ \\
\hline 4A & $0.1 \mathrm{M}$ sodium acetate $\mathrm{pH} 5$ & $15 \%$ MPD, 2\% PEG 4000 & $0.96 \mathrm{mM}$ & $0.87 \mathrm{mM}$ & $1.1: 1$ & $20^{\circ} \mathrm{C}$ \\
\hline 4B & $0.1 \mathrm{M}$ sodium acetate $\mathrm{pH} 5$ & 15\% MPD, 2\% PEG 4000 & $1.29 \mathrm{mM}$ & $0.68 \mathrm{mM}$ & $1.9: 1$ & $20^{\circ} \mathrm{C}$ \\
\hline $4 \mathrm{C}$ & $0.1 \mathrm{M}$ sodium acetate $\mathrm{pH} 5$ & $15 \%$ MPD, $2 \%$ PEG 4000 & $1.57 \mathrm{mM}$ & $0.54 \mathrm{mM}$ & $2.9: 1$ & $20^{\circ} \mathrm{C}$ \\
\hline $\mathbf{5 A}$ & $0.1 \mathrm{M}$ HEPES $\mathrm{pH} 7$ & $10 \%$ PEG 6000 & $0.96 \mathrm{mM}$ & $0.87 \mathrm{mM}$ & $1.1: 1$ & $20^{\circ} \mathrm{C}$ \\
\hline 5B & $0.1 \mathrm{M}$ HEPES $\mathrm{pH} 7$ & $10 \%$ PEG 6000 & $1.29 \mathrm{mM}$ & $0.68 \mathrm{mM}$ & 1.9:1 & $20^{\circ} \mathrm{C}$ \\
\hline $5 C$ & $0.1 \mathrm{M}$ HEPES pH 7 & $10 \%$ PEG 6000 & $1.57 \mathrm{mM}$ & $0.54 \mathrm{mM}$ & 2.9:1 & $20^{\circ} \mathrm{C}$ \\
\hline 6A & $0.1 \mathrm{M}$ Tris $\mathrm{pH} 8.5$ & $30 \%$ PEG 300 & $0.96 \mathrm{mM}$ & $0.87 \mathrm{mM}$ & $1.1: 1$ & $20^{\circ} \mathrm{C}$ \\
\hline $6 B$ & $0.1 \mathrm{M}$ Tris $\mathrm{pH} 8.5$ & $30 \%$ PEG 300 & $1.29 \mathrm{mM}$ & $0.68 \mathrm{mM}$ & $1.9: 1$ & $20^{\circ} \mathrm{C}$ \\
\hline
\end{tabular}




\begin{tabular}{|l|llllll|}
\hline 6C & $0.1 \mathrm{M}$ Tris pH 8.5 & $30 \%$ PEG 300 & $1.57 \mathrm{mM}$ & $0.54 \mathrm{mM}$ & $2.9: 1$ & $20{ }^{\circ} \mathrm{C}$ \\
\hline 7A & $0.1 \mathrm{M}$ Tris $\mathrm{pH} 8.5$ & $12 \%$ PEG 4000 & $0.96 \mathrm{mM}$ & $0.87 \mathrm{mM}$ & $1.1: 1$ & $20^{\circ} \mathrm{C}$ \\
\hline
\end{tabular}

Table 3.10: Initial screening conditions of mammalian Atg5 Atg16L1(1-70) with IP $(1,3)_{2}$ (continued).

\begin{tabular}{|c|c|c|c|c|c|c|}
\hline & Buffer & Precipitant (w/v)/ Salt & {$\left[\mathrm{IP}(\mathbf{1}, \mathbf{3})_{2}\right]$} & [Protein] & $\begin{array}{l}\text { Molar } \\
\text { Ratio }\end{array}$ & Temp. \\
\hline $7 \mathrm{~B}$ & $0.1 \mathrm{M}$ Tris $\mathrm{pH} 8.5$ & $12 \%$ PEG 4000 & $1.29 \mathrm{mM}$ & $0.68 \mathrm{mM}$ & $1.9: 1$ & $20^{\circ} \mathrm{C}$ \\
\hline $7 \mathrm{C}$ & $0.1 \mathrm{M}$ Tris $\mathrm{pH} 8.5$ & $12 \%$ PEG 4000 & $1.57 \mathrm{mM}$ & $0.54 \mathrm{mM}$ & $2.9: 1$ & $20^{\circ} \mathrm{C}$ \\
\hline 8 & $0.1 \mathrm{M}$ sodium citrate $\mathrm{pH} 5$ & $8 \%$ PEG 8000 & $1.36 \mathrm{mM}$ & $0.68 \mathrm{mM}$ & $2: 1$ & $4{ }^{\circ} \mathrm{C}$ \\
\hline 9 & 0.1 M HEPES $p H 7$ & $\begin{array}{c}\text { 15\% PEG 4000, } \\
0.1 \text { M magnesium chloride }\end{array}$ & $1.29 \mathrm{mM}$ & $0.68 \mathrm{mM}$ & $1.9: 1$ & $20^{\circ} \mathrm{C}$ \\
\hline 10 & 0.1 M HEPES $\mathrm{pH} 7.5$ & $\begin{array}{c}\text { 15\% PEG 6000, } \\
0.1 \mathrm{M} \text { potassium chloride }\end{array}$ & $\begin{array}{l}0.83 \\
\mathrm{mM}^{*}\end{array}$ & $0.75 \mathrm{mM}$ & 1.1:1 & $20^{\circ} \mathrm{C}$ \\
\hline 11 & $0.1 \mathrm{M}$ MES pH 6.5 & $\begin{array}{c}7 \% \text { PEG } 4000, \\
10 \%(\mathrm{v} / \mathrm{v}) \text { isopropanol }\end{array}$ & $1.36 \mathrm{mM}$ & $0.68 \mathrm{mM}$ & $2: 1$ & $4{ }^{\circ} \mathrm{C}$ \\
\hline 12 & $0.1 \mathrm{M}$ MES pH 6 & $\begin{array}{c}7 \% \text { PEG } 4000, \\
10 \%(\mathrm{v} / \mathrm{v}) \text { isopropanol }\end{array}$ & $1.25 \mathrm{mM}$ & $0.6 \mathrm{mM}$ & $2.1: 1$ & $4{ }^{\circ} \mathrm{C}$ \\
\hline 13A & $0.1 \mathrm{M}$ HEPES sodium $\mathrm{pH} 8$ & $\begin{array}{c}7 \% \text { PEG } 4000, \\
10 \%(\mathrm{v} / \mathrm{v}) \text { isopropanol }\end{array}$ & $1.36 \mathrm{mM}$ & $0.68 \mathrm{mM}$ & $2: 1$ & $20^{\circ} \mathrm{C}$ \\
\hline 13B & $0.1 \mathrm{M}$ HEPES sodium $\mathrm{pH} 8$ & $\begin{array}{c}7 \% \text { PEG } 4000, \\
10 \%(\mathrm{v} / \mathrm{v}) \text { isopropanol }\end{array}$ & $1.25 \mathrm{mM}$ & $0.6 \mathrm{mM}$ & $2.1: 1$ & $20^{\circ} \mathrm{C}$ \\
\hline $13 \mathrm{C}$ & $0.1 \mathrm{M}$ HEPES sodium $\mathrm{pH} 8$ & $\begin{array}{c}7 \% \text { PEG } 4000, \\
10 \%(\mathrm{v} / \mathrm{v}) \text { isopropanol }\end{array}$ & $\begin{array}{l}1.38 \\
\mathrm{mM}^{*}\end{array}$ & $0.45 \mathrm{mM}$ & $3: 1$ & $20^{\circ} \mathrm{C}$ \\
\hline 14 & $0.1 \mathrm{M}$ HEPES $\mathrm{pH} 7$ & $\begin{array}{c}5 \% \text { PEG } 4000, \\
10 \%(\mathrm{v} / \mathrm{v}) \text { isopropanol }\end{array}$ & $1.36 \mathrm{mM}$ & $0.68 \mathrm{mM}$ & $2: 1$ & $20^{\circ} \mathrm{C}$ \\
\hline 15A & $0.1 \mathrm{M}$ Tris $\mathrm{pH} 9$ & $\begin{array}{c}\text { 6\% PEG 4000, } \\
10 \%(\mathrm{v} / \mathrm{v}) \text { isopropanol }\end{array}$ & $1.3 \mathrm{mM}^{*}$ & $0.65 \mathrm{mM}$ & $2: 1$ & $20^{\circ} \mathrm{C}$ \\
\hline 15B & $0.1 \mathrm{M}$ Tris $\mathrm{pH} 9$ & $\begin{array}{c}6 \% \text { PEG } 4000, \\
10 \%(\mathrm{v} / \mathrm{v}) \text { isopropanol }\end{array}$ & $1.5 \mathrm{mM}^{*}$ & $0.5 \mathrm{mM}$ & $3: 1$ & $20^{\circ} \mathrm{C}$ \\
\hline
\end{tabular}

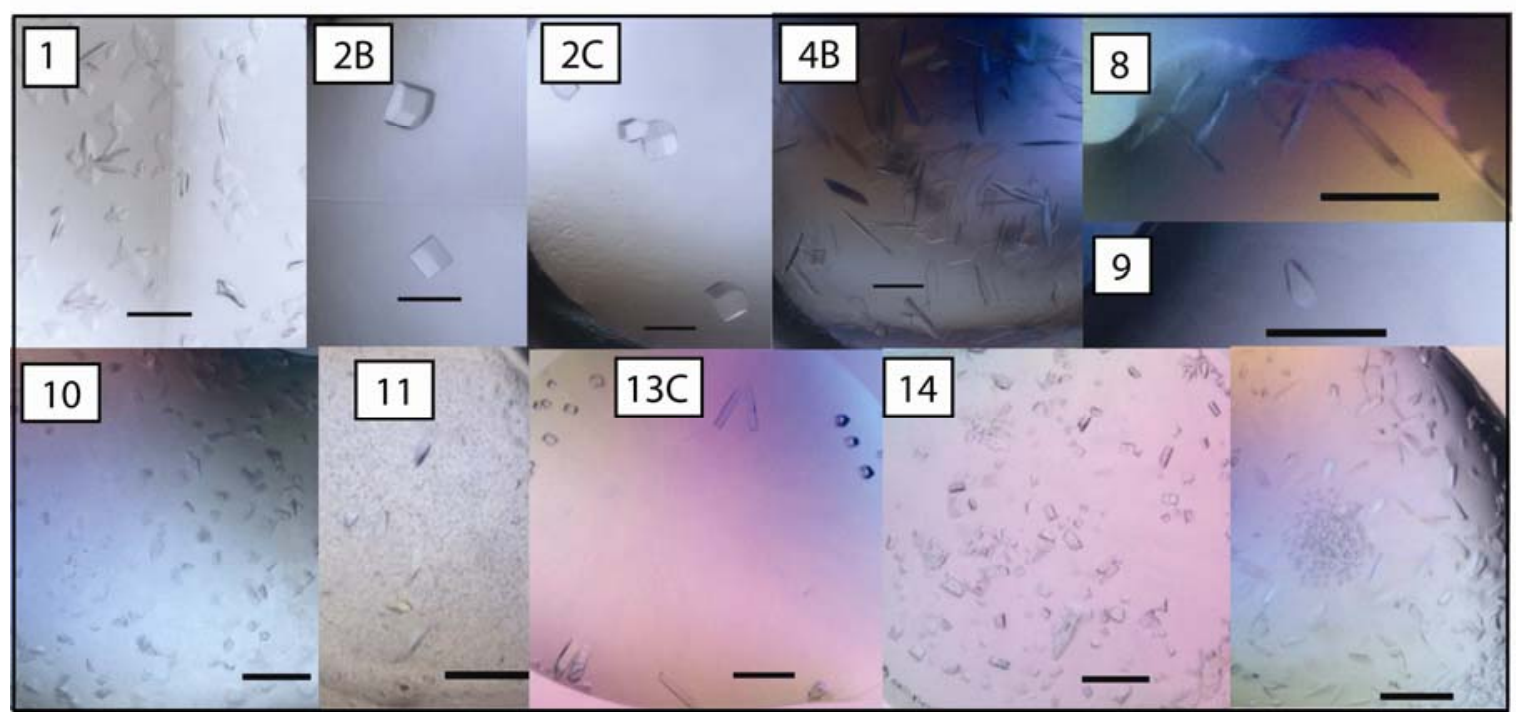

Figure 3.60: Crystals of the mammalian Atg5 Atg16L1(1-70) complex co-crystallized with D-myoInositol-(1,3)-diphosphate (sodium salt) $\left[\mathrm{IP}(\mathbf{1}, \mathbf{3})_{2}\right]$. Numbers correspond to the crystallization conditions given in Table 3.10. Scale bar $=200 \mu \mathrm{m}$.

Since attempts to reproduce crystal growth in 24-well Linbro plates failed, 96-well plates were used for all subsequent optimization screens. Different PEG concentrations, protein and $\mathrm{IP}(1,3)_{2}$ concentrations were tested at different $\mathrm{pH}$ values. In addition, further 
crystallization trials were set-up with the Additive screen (HR2-428), Silver Bullets (HR2078) and Silver Bullets Bio (HR2-080) from Hampton Research. The Additive screen contains 96 different organic and inorganic compounds and allows rapid testing of their influence on crystal growth. The Silver Bullets and Silver Bullets Bio screens are libraries of small molecules, which stabilize intermolecular interactions and could thereby promote lattice formation (McPherson 2006). These screens were also set-up with different combinations of buffer, precipitant, and protein concentrations and this helped to improve the crystal size. Pictures of these crystals are shown in Figure 3.61 and crystallization conditions are summarized in Table 3.11. Crystal growth typically took three days for the Silver Bullets screens and up to two weeks for the Additive screen plates.

Crystals of Atg5 Atg16L1(1-70) $+\operatorname{IP}(1,3)_{2}$ grown either in the presence or absence of additives were flash-cooled in liquid nitrogen after being equilibrated in the cryoprotectants using a three-step serial dilution from the mother liquor to $100 \%$ cryoprotectant. Cryoprotectants were composed of reservoir solution supplemented with either $20-30 \%$ ethylene glycol, 10\% (w/v) xylitol/10\% (w/v) sucrose, 30\% PEG 400, or $30 \%$ ethylene glycol containing $1 \mathrm{mM} \mathrm{IP}(1,3)_{2}$. Alternatively crystals were slowly streaked through perfluoropolyether oil to remove excess liquid before flash-cooling.

Diffraction data of cryo-cooled crystals were collected at $100 \mathrm{~K}$ on a Pilatus detector at the Swiss Light Source (Switzerland) beamline PXII. The growth conditions, cryoprotectants and diffraction limits of the 10 best tested crystals are summarized in Table 3.11 and a selection of their pictures are shown in Figure 3.61. The additive screens improved the diffraction quality of the crystals. However, these crystals still diffracted only weakly and the highest resolution observed was $6 \AA$.

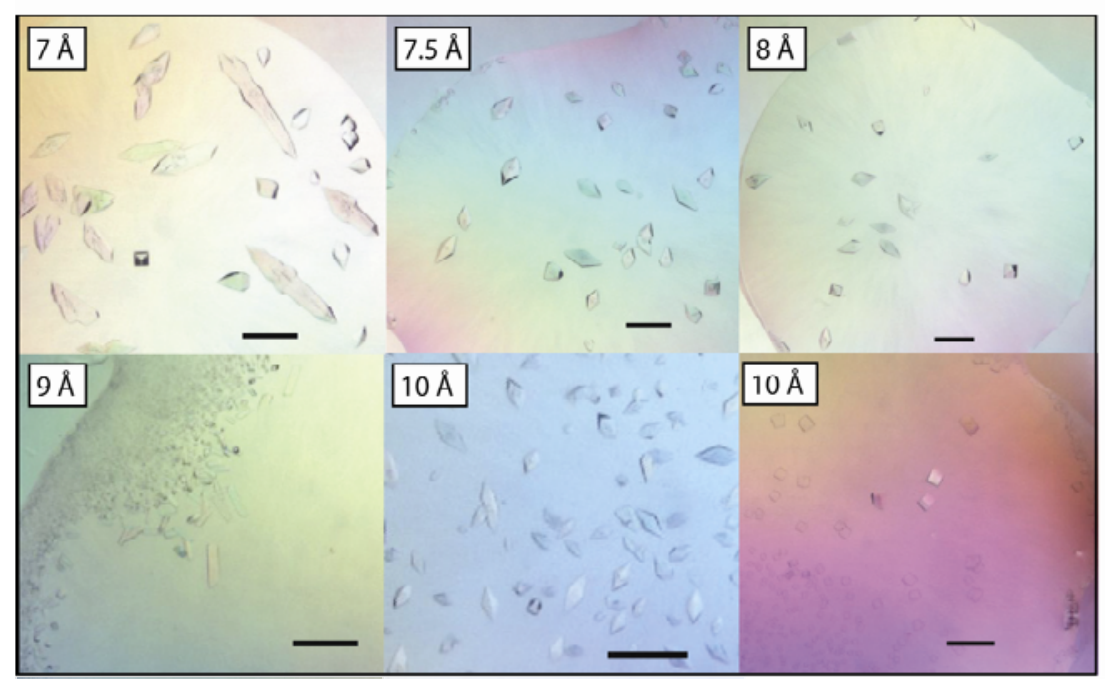


Figure 3.61: Crystals of the mammalian Atg5 Atg16L1(1-70) complex co-crystallized with IP(1,3) and additives. Numbers correspond to the diffraction resolution limit. Scale bar $=200 \mu \mathrm{m}$. 
Table 3.11. Crystallization conditions, cryoprotectants, and diffraction resolution data for the 10 best crystals of mammalian $\operatorname{Atg5} \sim \operatorname{Atg} 16(1-70)$ with IP(1,3)2

\begin{tabular}{|c|c|c|c|c|c|c|c|c|}
\hline $\begin{array}{c}\text { Crystallization } \\
\text { Condition }\end{array}$ & Additive composition & $\begin{array}{c}\text { IP }(1,3)_{2} \\
\text { Conc. }\end{array}$ & $\begin{array}{l}\text { Prot. } \\
\text { Conc. }\end{array}$ & Ratio & $\begin{array}{l}\text { Drop } \\
\text { setup }\end{array}$ & Temp. & $\begin{array}{c}\text { Cryo- } \\
\text { protectant }\end{array}$ & $\begin{array}{l}\text { Diff. } \\
\text { Res. }\end{array}$ \\
\hline $\begin{array}{c}0.1 \mathrm{M} \text { Tris } \mathrm{pH} 9 \\
6 \% \text { PEG } 4000\end{array}$ & $\begin{array}{l}0.20 \% \mathrm{w} / \mathrm{v} \text { Adenosine 5'-triphosphate disodium salt hydrate, } 0.20 \% \mathrm{w} / \mathrm{v} 2 \text { '- } \\
\text { Deoxycytidine 5'-monophosphate sodium salt, } 0.20 \% \mathrm{w} / \mathrm{v} \text { B-Estradiol, } 0.20 \% \mathrm{w} / \mathrm{v} \mathrm{D} \text { - } \\
(+) \text {-Galactose, } 0.20 \% \mathrm{w} / \mathrm{v} \text { 2'-Deoxyguanosine hydrate }\end{array}$ & $\begin{array}{l}1.38 \\
\mathrm{mM}\end{array}$ & $\begin{array}{l}0.55 \\
\mathrm{mM}\end{array}$ & $2.5: 1$ & $\begin{array}{l}\text { 100:100: } \\
\text { 20nL }\end{array}$ & $20^{\circ} \mathrm{C}$ & $\begin{array}{l}20 \% \text { ethylene } \\
\text { glycol }\end{array}$ & $6 \AA$ \\
\hline $\begin{array}{l}0.1 \mathrm{M} \text { HEPES } \mathrm{pH} \\
7,5 \% \text { PEG } 4000\end{array}$ & $\begin{array}{l}0.20 \% \mathrm{w} / \mathrm{v} \text { Sodium pyrophosphate tetrabasic decahydrate, } 0.20 \% \mathrm{w} / \mathrm{v} \mathrm{D}-(-)-\text { Ribose, } \\
0.20 \% \mathrm{w} / \mathrm{v} \text { Phytic acid sodium salt hydrate, } 0.20 \% \mathrm{w} / \mathrm{v} \text { Adenosine } 3 \text { ', } 5 \text { '-cyclic } \\
\text { monophosphate sodium salt monohydrate, } 0.04 \% \mathrm{w} / \mathrm{v} \text { Acetyl coenzyme A sodium salt }\end{array}$ & $1.2 \mathrm{mM}$ & $\begin{array}{l}0.45 \\
\mathrm{mM}\end{array}$ & $2.7: 1$ & $\begin{array}{l}\text { 200:200: } \\
\text { 40nL }\end{array}$ & $20^{\circ} \mathrm{C}$ & $\begin{array}{c}10 \% \text { sucrose, } \\
10 \% \text { xylitol }\end{array}$ & $7 \AA$ \\
\hline $\begin{array}{c}0.1 \mathrm{M} \text { HEPES } \mathrm{pH} \\
7,5 \% \text { PEG } 4000\end{array}$ & see above & $1.2 \mathrm{mM}$ & $\begin{array}{l}0.45 \\
\mathrm{mM}\end{array}$ & $2.7: 1$ & $\begin{array}{c}\text { 200:200: } \\
\text { 40nL }\end{array}$ & $20^{\circ} \mathrm{C}$ & $\begin{array}{l}1 \mathrm{mM} \mathrm{IP}(1,3)_{2} \\
\text { in } 30 \% \text { ethylene } \\
\text { glycol }\end{array}$ & $\begin{array}{l}7.5 \\
\AA\end{array}$ \\
\hline $\begin{array}{l}0.1 \text { M HEPES } \\
\text { sodium pH } 8,7 \% \\
\text { PEG } 4000\end{array}$ & $\begin{array}{l}0.16 \% \mathrm{w} / \mathrm{v} \text { D-3-Phosphoglyceric acid disodium salt, } 0.16 \% \mathrm{w} / \mathrm{v} \text { D-Fructose } 1,6- \\
\text { diphosphate trisodium salt octahydrate, } 0.16 \% \mathrm{w} / \mathrm{v} \text { D-Glucose } 6-\text { phosphate, } 0.16 \% \\
\mathrm{w} / \mathrm{v} \text { L-O-Phosphoserine, } 0.16 \% \mathrm{w} / \mathrm{v} \text { O-Phospho-L-tyrosine, } 0.16 \% \mathrm{w} / \mathrm{v} \text { Phytic acid } \\
\text { sodium salt hydrate }\end{array}$ & $1.2 \mathrm{mM}$ & $\begin{array}{l}0.45 \\
\mathrm{mM}\end{array}$ & $2.7: 1$ & $\begin{array}{l}\text { 200:200: } \\
\text { 40nL }\end{array}$ & $20^{\circ} \mathrm{C}$ & $\begin{array}{c}30 \% \text { ethylene } \\
\text { glycol }\end{array}$ & $\begin{array}{l}7.5 \\
\AA\end{array}$ \\
\hline $\begin{array}{l}0.1 \mathrm{M} \mathrm{HEPES} \\
\text { sodium } \mathrm{pH} 8,7 \% \\
\text { PEG } 4000\end{array}$ & see above & $1.2 \mathrm{mM}$ & $\begin{array}{l}0.45 \\
\mathrm{mM}\end{array}$ & $2.7: 1$ & $\begin{array}{l}\text { 200:200: } \\
\text { 40nL }\end{array}$ & $20^{\circ} \mathrm{C}$ & $\begin{array}{l}1 \mathrm{mM} \mathrm{IP}(1,3)_{2} \\
\text { in } 30 \% \text { ethylene } \\
\text { glycol }\end{array}$ & $8 \AA$ \\
\hline $\begin{array}{l}\text { 0.1 M HEPES } \\
\text { sodium pH } 8,7 \% \\
\text { PEG } 4000\end{array}$ & $\begin{array}{l}0.07 \% \mathrm{w} / \mathrm{v} \text { Barbituric acid, } 0.07 \% \mathrm{w} / \mathrm{v} \text { Benzidine, } 0.07 \% \mathrm{w} / \mathrm{v} \text { Cystathionine, } 0.07 \% \\
\mathrm{w} / \mathrm{v} \text { L-Canavanine, } 0.07 \% \mathrm{w} / \mathrm{v} \text { L-Carnitine hydrochloride, } 0.07 \% \mathrm{w} / \mathrm{v} \text { L-Cystine, } \\
0.07 \% \mathrm{w} / \mathrm{v} \text { Mellitic acid }\end{array}$ & $1 \mathrm{mM}$ & $\begin{array}{l}0.34 \\
\mathrm{mM}\end{array}$ & $2.9: 1$ & $\begin{array}{l}\text { 200:200: } \\
\text { 40nL }\end{array}$ & $20^{\circ} \mathrm{C}$ & $\begin{array}{l}1 \mathrm{mM} \mathrm{IP}(1,3)_{2} \\
\text { in } 30 \% \text { ethylene } \\
\text { glycol }\end{array}$ & $9 \AA$ \\
\hline $\begin{array}{l}0.1 \mathrm{M} \mathrm{HEPES} \\
\text { sodium } \mathrm{pH} 8,7 \% \\
\text { PEG } 4000\end{array}$ & $\begin{array}{l}0.2 \% \mathrm{w} / \mathrm{v} \text { D-Fructose } 1,6 \text {-diphosphate trisodium salt octahydrate, } 0.2 \% \mathrm{w} / \mathrm{v} \text { Glycerol } \\
\text { phosphate disodium salt hydrate, } 0.2 \% \mathrm{w} / \mathrm{v} \text { L-O-Phosphoserine, } 0.2 \% \mathrm{w} / \mathrm{v} \mathrm{O}- \\
\text { Phospho-L-tyrosine, } 0.2 \% \mathrm{w} / \mathrm{v} \text { Phytic acid sodium salt hydrate }\end{array}$ & $1 \mathrm{mM}$ & $\begin{array}{c}0.4 \\
\mathrm{mM}\end{array}$ & $2.5: 1$ & $\begin{array}{c}\text { 200:200: } \\
\text { 40nL }\end{array}$ & $20^{\circ} \mathrm{C}$ & $\begin{array}{c}10 \% \text { sucrose } \\
10 \% \text { xylitol }\end{array}$ & $10 \AA$ \\
\hline $\begin{array}{l}0.1 \mathrm{M} \text { HEPES } \mathrm{pH} \\
7,5 \% \text { PEG } 4000\end{array}$ & $\begin{array}{l}0.16 \% \mathrm{w} / \mathrm{v} 3 \text {-Aminobenzenesulfonic acid, } 0.16 \% \mathrm{w} / \mathrm{v} 5 \text {-Sulfosalicylic acid dihydrate, } \\
0.16 \% \mathrm{w} / \mathrm{v} \text {-Coumaric acid, } 0.16 \% \mathrm{w} / \mathrm{v} \text { PIPES, } 0.16 \% \mathrm{w} / \mathrm{v} \text { Terephthalic acid, } 0.16 \% \\
\mathrm{w} / \mathrm{v} \text { Vanillic acid }\end{array}$ & $0.6 \mathrm{mM}$ & $\begin{array}{c}0.2 \\
\mathrm{mM}\end{array}$ & 3.1 & $\begin{array}{l}\text { 200:200: } \\
\text { 40nL }\end{array}$ & $20^{\circ} \mathrm{C}$ & $\begin{array}{l}1 \mathrm{mM} \mathrm{IP}(1,3)_{2} \\
\text { in } 30 \% \text { ethylene } \\
\text { glycol }\end{array}$ & $10 \AA$ \\
\hline $\begin{array}{l}0.1 \text { M HEPES } \\
\text { sodium pH } 8,7 \% \\
\text { PEG } 4000\end{array}$ & $\begin{array}{l}0.16 \% \mathrm{w} / \mathrm{v} \text { D-3-Phosphoglyceric acid disodium salt, } 0.16 \% \mathrm{w} / \mathrm{v} \text { D-Fructose } 1,6- \\
\text { diphosphate trisodium salt octahydrate, } 0.16 \% \mathrm{w} / \mathrm{v} \text { D-Glucose } 6-\text { phosphate, } 0.16 \% \\
\mathrm{w} / \mathrm{v} \text { L-O-Phosphoserine, } 0.16 \% \mathrm{w} / \mathrm{v} \text { O-Phospho-L-tyrosine, } 0.16 \% \mathrm{w} / \mathrm{v} \text { Phytic acid } \\
\text { sodium salt hydrate }\end{array}$ & $1.2 \mathrm{mM}$ & $\begin{array}{l}0.45 \\
\mathrm{mM}\end{array}$ & $2.7: 1$ & $\begin{array}{c}\text { 200:200: } \\
\text { 40nL }\end{array}$ & $20^{\circ} \mathrm{C}$ & $\begin{array}{l}\text { Perfluoropoly- } \\
\text { ether oil }\end{array}$ & $10 \AA$ \\
\hline $\begin{array}{l}0.1 \mathrm{M} \text { HEPES } \mathrm{pH} \\
7,5 \% \text { PEG } 4000\end{array}$ & $\begin{array}{l}0.20 \% \mathrm{w} / \mathrm{v} \text { Sodium pyrophosphate tetrabasic decahydrate, } 0.20 \% \mathrm{w} / \mathrm{v} \mathrm{D}-(-) \text {-Ribose, } \\
0.20 \% \mathrm{w} / \mathrm{v} \text { Phytic acid sodium salt hydrate, } 0.20 \% \mathrm{w} / \mathrm{v} \text { Adenosine } 3 \text { ', } 5 \text { '-cyclic } \\
\text { monophosphate sodium salt monohydrate, } 0.04 \% \mathrm{w} / \mathrm{v} \text { Acetyl coenzyme A sodium salt }\end{array}$ & $1.2 \mathrm{mM}$ & $\begin{array}{l}0.45 \\
\mathrm{mM}\end{array}$ & $2.7: 1$ & $\begin{array}{l}\text { 200:200: } \\
\text { 40nL }\end{array}$ & $20^{\circ} \mathrm{C}$ & $\begin{array}{c}10 \% \text { sucrose, } \\
10 \% \text { xylitol }\end{array}$ & $10 \AA$ \\
\hline
\end{tabular}

Drop setup= protein: reservoir 


\section{Murine Atg5 Atg16L1(1-106) and Atg5 Atg16L1(1-113)}

At the same time when the mammalian Atg5 Atg16L1(1-70) complex was being co-crystallized with IP $(1,3)_{2}$, I found that complex oligomerization increases the PIP binding affinity of Atg5 Atg16L1 (Figures 3.40 and 3.41). Therefore, I also tried to cocrystallize the murine Atg5 Atg16L1(1-106) and Atg5 Atg16L1(1-113) complexes with $\operatorname{IP}(1,3)_{2}$.

First, the commercial screens Classics, ClassicsII, Protein Complex, Cations, PACT, JCSG+, MbClass, MbClass II, Nucleix, Classics Lite, and ComPAS suites from Qiagen; SaltRx and Index from Hampton; and Wizard I\&II and Wizard III\&IV from Emerald Biosystems were set-up for both complexes at different protein concentrations. Next, self-made screens were set up at different $\mathrm{pH}$ values using different buffers and testing out various concentrations of the different precipitants. Both 96-well plates (sitting drops) and 24-well Linbro plates (hanging drops) were used for optimization. In addition to setting the plates up at both $20{ }^{\circ} \mathrm{C}$ and $4{ }^{\circ} \mathrm{C}$, the protein and $\operatorname{IP}(1,3)_{2}$ concentrations, their ratio, and buffer and salt composition of the $\operatorname{IP}(1,3)_{2}$ were also varied. Also the Additive screen was used for optimization trials.

Crystals of the Atg5 Atg16L1(1-106) complex with IP(1,3) grew within 1 to 2 weeks and could be optimized in 24-well Linbro plates where larger drops can be pipetted enabling larger crystals to grow. Streak seeding was also tried in an attempt to obtain larger crystals. Larger crystals grew when the drops were covered with Al's oil, a 1:1 mixture of paraffin oil and silicon oil. The oil acts as diffusion barrier between the reservoir and the drop and slows down equilibration (Chayen 1997). In contrast, the Atg5 Atg16L1(1-113) complex co-crystallized with IP(1,3) $)_{2}$ took about two months to grow and although they grew in three drops in a 24 -well Linbro plate, these crystals were difficult to reproduce.

It was also tried to crystallize Atg5 Atg16L1(1-106) in the presence of $200 \mathrm{mM}$ $\mathrm{Na}_{2} \mathrm{SO}_{4}$. The idea was that the sulfate group might bind in the same pocket as the phosphate groups of $\operatorname{IP}(1,3)_{2}$ revealing the binding site for the PI(3)P head group. The Protein Complex, Classics, and Classics II suite commercial screens from Qiagen were set up at $4{ }^{\circ} \mathrm{C}$ and yielded one hit (Table 3.12). The crystallization conditions for Atg5 Atg16L1(1-113) and Atg5 Atg16L1(1-106) complexes with $\operatorname{IP}(1,3)_{2}$ are summarized in Table 3.12 and pictures of the crystals are shown in Figure 3.62. 
Table 3.12: Crystallization conditions for mammalian Atg5 Atg16L1(1-106) and Atg5 Atg16L1(1-113) with IP(1,3)

\begin{tabular}{|c|c|c|c|c|c|c|c|c|}
\hline & & Buffer & $\begin{array}{c}\text { Precipitant }(w / v) / \\
\text { Other }\end{array}$ & {$\left[\mathrm{IP}(\mathbf{1}, \mathbf{3})_{2}\right]$} & [Protein] & $\begin{array}{l}\text { Molar } \\
\text { Ratio } \\
\end{array}$ & Temp. & $\begin{array}{l}\text { Drop } \\
\text { setup }\end{array}$ \\
\hline 1 & 113 & 0.1 M HEPES pH 7.5 & $\begin{array}{c}15 \% \text { PEG 6000, } \\
0.1 \mathrm{M} \mathrm{KCl}\end{array}$ & $0.85 \mathrm{mM}$ & $0.77 \mathrm{mM}$ & $1.1: 1$ & $20^{\circ} \mathrm{C}$ & $\begin{array}{c}\text { 100: } \\
100 \mathrm{~nL}\end{array}$ \\
\hline 2 & 113 & $0.1 \mathrm{M}$ HEPES pH 7 & $10 \%$ PEG 6000 & $1.33 \mathrm{mM}$ & $0.59 \mathrm{mM}$ & $2.25: 1$ & $4{ }^{\circ} \mathrm{C}$ & $\begin{array}{c}100: \\
100 \mathrm{~nL}\end{array}$ \\
\hline 3 & 113 & $0.1 \mathrm{M}$ MES pH 6 & $11 \%$ PEG 6000 & $0.6 \mathrm{mM}$ & $0.2 \mathrm{mM}$ & $3: 1$ & $4{ }^{\circ} \mathrm{C}$ & $1: 1 \mu \mathrm{L}$ \\
\hline 4 & 113 & $0.1 \mathrm{M}$ MES pH 6.5 & $11 \%$ PEG 6000 & $0.9 \mathrm{mM}$ & $0.3 \mathrm{mM}$ & $3: 1$ & $4{ }^{\circ} \mathrm{C}$ & $1: 1 \mu \mathrm{L}$ \\
\hline 5 & 113 & $0.1 \mathrm{M}$ MES pH 6.5 & $13 \%$ PEG 6000 & $0.9 \mathrm{mM}$ & $0.3 \mathrm{mM}$ & $3: 1$ & $4{ }^{\circ} \mathrm{C}$ & $1: 1 \mu \mathrm{L}$ \\
\hline 6 & 106 & $0.1 \mathrm{M}$ HEPES $\mathrm{pH} 7$ & $8 \%$ PEG 8000 & $1.33 \mathrm{mM}$ & $0.59 \mathrm{mM}$ & $2.25: 1$ & $4{ }^{\circ} \mathrm{C}$ & $\begin{array}{c}100: \\
100 \mathrm{~nL}\end{array}$ \\
\hline 7 & 106 & $0.1 \mathrm{M}$ MES pH 6.5 & $8 \%$ PEG 8000 & $1.47 \mathrm{mM}$ & $0.49 \mathrm{mM}$ & $3: 1$ & $4^{\circ} \mathrm{C}$ & $1: 1 \mu \mathrm{L}$ \\
\hline 8 & 106 & $0.1 \mathrm{M}$ MES pH 6.5 & $10 \%$ PEG 8000 & $1.47 \mathrm{mM}$ & $0.49 \mathrm{mM}$ & $3: 1$ & $4{ }^{\circ} \mathrm{C}$ & $1: 1 \mu \mathrm{L}$ \\
\hline 9 & 106 & $0.1 \mathrm{M}$ MES pH 6.5 & $12 \%$ PEG 8000 & $1.47 \mathrm{mM}$ & $0.49 \mathrm{mM}$ & $3: 1$ & $4{ }^{\circ} \mathrm{C}$ & $1: 1 \mu \mathrm{L}$ \\
\hline 10 & 106 & $0.1 \mathrm{M}$ HEPES $\mathrm{pH} 7$ & $6 \%$ PEG 8000 & $1.33 \mathrm{mM}$ & $0.59 \mathrm{mM}$ & $2.25: 1$ & $4{ }^{\circ} \mathrm{C}$ & $1: 1 \mu \mathrm{L}$ \\
\hline $11 \mathrm{~A}$ & 106 & $0.1 \mathrm{M}$ HEPES pH 7 & $8 \%$ PEG 8000 & $1.33 \mathrm{mM}$ & $0.59 \mathrm{mM}$ & $2.25: 1$ & $4{ }^{\circ} \mathrm{C}$ & $1: 1 \mu \mathrm{L}$ \\
\hline 11B & 106 & $0.1 \mathrm{M}$ HEPES pH 7 & $8 \%$ PEG 8000 & $1.47 \mathrm{mM}$ & $0.49 \mathrm{mM}$ & $3: 1$ & $4{ }^{\circ} \mathrm{C}$ & $1: 1 \mu \mathrm{L}$ \\
\hline 12 & 106 & $0.1 \mathrm{M}$ HEPES pH 7 & $10 \%$ PEG 8000 & $1.33 \mathrm{mM}$ & $0.59 \mathrm{mM}$ & $2.25: 1$ & $4{ }^{\circ} \mathrm{C}$ & $1: 1 \mu \mathrm{L}$ \\
\hline 13 & 106 & 0.1 M HEPES pH 7 & $12 \%$ PEG 8000 & $1.33 \mathrm{mM}$ & $0.59 \mathrm{mM}$ & $2.25: 1$ & $4{ }^{\circ} \mathrm{C}$ & $1: 1 \mu \mathrm{L}$ \\
\hline $14 \mathrm{~A}$ & 106 & $0.1 \mathrm{M}$ HEPES $\mathrm{pH} 7.5$ & $12 \%$ PEG 8000 & $1.33 \mathrm{mM}$ & $0.59 \mathrm{mM}$ & $2.25: 1$ & $4{ }^{\circ} \mathrm{C}$ & $1: 1 \mu \mathrm{L}$ \\
\hline 14B & 106 & $0.1 \mathrm{M}$ HEPES $\mathrm{pH} 7.5$ & $12 \%$ PEG 8000 & $1.47 \mathrm{mM}$ & $0.49 \mathrm{mM}$ & $2.25: 1$ & $4{ }^{\circ} \mathrm{C}$ & $1: 1 \mu \mathrm{L}$ \\
\hline 15 & 106 & 0.1 M HEPES pH 7.5 & 14\% PEG 8000 & $1.47 \mathrm{mM}$ & $0.49 \mathrm{mM}$ & $2.25: 1$ & $4{ }^{\circ} \mathrm{C}$ & $1: 1 \mu \mathrm{L}$ \\
\hline 16A & 106 & 0.1 M HEPES pH 7 & $\begin{array}{l}8 \% \text { PEG } 8000, \\
250 \mu \mathrm{L} \text { Al's oil } \\
\end{array}$ & $1.5 \mathrm{mM}$ & $0.5 \mathrm{mM}$ & $3: 1$ & $4{ }^{\circ} \mathrm{C}$ & $1: 1 \mu \mathrm{L}$ \\
\hline $16 B$ & 106 & $0.1 \mathrm{M}$ HEPES pH 7 & $\begin{array}{l}\text { 8\% PEG } 8000, \\
250 \mu \mathrm{L} \text { Al's oil }\end{array}$ & $1.2 \mathrm{mM}$ & $0.4 \mathrm{mM}$ & $3: 1$ & $4{ }^{\circ} \mathrm{C}$ & $1: 1 \mu \mathrm{L}$ \\
\hline 17 & 106 & 0.1 M HEPES pH 7 & $\begin{array}{l}\text { 8\% PEG 8000, } \\
500 \mu \mathrm{L} \text { Al's oil } \\
\end{array}$ & $1.2 \mathrm{mM}$ & $0.4 \mathrm{mM}$ & $3: 1$ & $4{ }^{\circ} \mathrm{C}$ & $1: 1 \mu \mathrm{L}$ \\
\hline 18 & 106 & $0.1 \mathrm{M}$ MES pH 6.5 & $6 \%$ PEG 8000 & $1.75 \mathrm{mM}$ & $0.35 \mathrm{mM}$ & $5: 1$ & $4{ }^{\circ} \mathrm{C}$ & $1: 1 \mu \mathrm{L}$ \\
\hline 19 & 106 & $0.1 \mathrm{M}$ Tris $\mathrm{pH} 8.5$ & $\begin{array}{c}30 \%(\mathrm{v} / \mathrm{v}) \\
\text { isopropanol, } \\
0.2 \mathrm{M} \text { ammonium } \\
\text { acetate, } \\
0.2 \mathrm{M} \mathrm{Na}_{2} \mathrm{SO}_{4} \\
\end{array}$ & $\begin{array}{l}200 \mathrm{mM} \\
\mathrm{Na}_{2} \mathrm{SO}_{4} \\
\end{array}$ & $25 \mathrm{mg} / \mathrm{mL}$ & & $20^{\circ} \mathrm{C}$ & $\begin{array}{c}100: \\
100 \mathrm{~nL}\end{array}$ \\
\hline 20A & 106 & $\begin{array}{c}0.1 \mathrm{M} \text { HEPES } \\
\text { sodium pH } 7\end{array}$ & $12 \%$ PEG 8000 & $1.34 \mathrm{mM}$ & $0.55 \mathrm{mM}$ & $2.4: 1$ & $4^{\circ} \mathrm{C}$ & $1: 1 \mu \mathrm{L}$ \\
\hline 20B & 106 & $\begin{array}{c}0.1 \mathrm{M} \text { HEPES } \\
\text { sodium } \mathrm{pH} 7 \\
\end{array}$ & $12 \%$ PEG 8000 & $1.34 \mathrm{mM}$ & $0.55 \mathrm{mM}$ & $2.4: 1$ & $4{ }^{\circ} \mathrm{C}$ & $2: 2 \mu \mathrm{L}$ \\
\hline 21 & 106 & $0.1 \mathrm{M}$ HEPES $\mathrm{pH} 7$ & $\begin{array}{l}\text { 6\% PEG 8000, } \\
250 \mu \mathrm{L} \text { Al's oil }\end{array}$ & $1.2 \mathrm{mM}$ & $0.4 \mathrm{mM}$ & $3: 1$ & $4{ }^{\circ} \mathrm{C}$ & $2: 2 \mu \mathrm{L}$ \\
\hline 22 & 106 & $0.1 \mathrm{M}$ MES pH 6.5 & $\begin{array}{l}\text { 9\% PEG } 8000 \\
0.1 \mathrm{M} \text { spermine } \\
\text { tetrachloride }\end{array}$ & $1.5 \mathrm{mM}$ & $0.5 \mathrm{mM}$ & $3: 1$ & $4{ }^{\circ} \mathrm{C}$ & $\begin{array}{c}1.25: 1: . \\
25 \mu \mathrm{L}\end{array}$ \\
\hline 23 & 106 & 0.1 M HEPES pH 7 & $\begin{array}{l}8 \% \text { PEG } 8000, \\
5 \% \text { w/v polyvinyl- } \\
\text { pyrrolidone K15 }\end{array}$ & $1.49 \mathrm{mM}$ & $0.55 \mathrm{mM}$ & $2.7: 1$ & $4{ }^{\circ} \mathrm{C}$ & $\begin{array}{c}1.25: 1: . \\
25 \mu \mathrm{L}\end{array}$ \\
\hline \multicolumn{9}{|c|}{$\begin{array}{l}\text { Buffer of protein= } 0.2 \mathrm{M} \mathrm{NaCl}, 30 \mathrm{mM} \text { HEPES } \mathrm{pH} 7.5 \\
* \mathrm{IP}(1,3) 2 \text { adjusted to } 30 \mathrm{mM} \text { HEPES } \mathrm{pH} 7.5 \\
\text { Drop setup= protein: reservoir }\end{array}$} \\
\hline
\end{tabular}




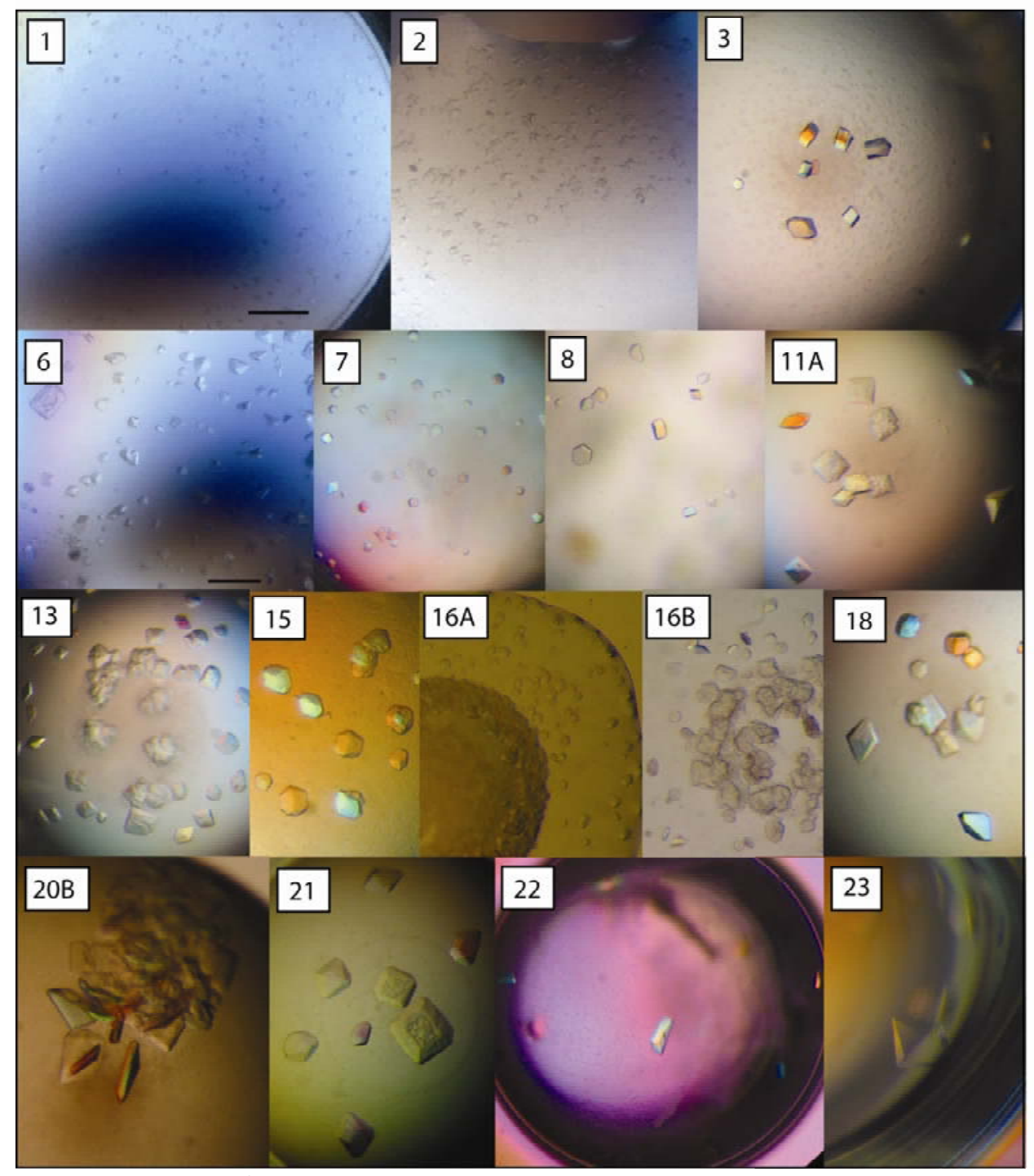

Figure 3.62: Crystals of the mammalian Atg5 Atg16L1(1-113) and Atg5 Atg16L1(1-106) complexes co-crystallized with IP(1,3) 2 . Numbers correspond to the crystallization conditions given in Table 3.12. Scale bar $=200 \mu \mathrm{m}$. 22-23 were crystallized in the presence of additives.

Crystals of mammalian Atg5 Atg16L1(1-113) + IP(1,3) $)_{2}$ and Atg5 Atg16L1(1106) $+\operatorname{IP}(1,3)_{2}$ were flash-cooled in liquid nitrogen after being serially transferred into cryoprotectants containing either 25 to $35 \%$ ethylene glycol or 25 to $30 \%$ glycerol. Alternatively crystals were slowly streaked through perfluoropolyether oil before flashcooling them. Diffraction data of cryo-cooled crystals were collected at $100 \mathrm{~K}$ on a Pilatus detector at the Swiss Light Source (Switzerland) beamline PXII. Approximately 50 crystals were tested. The crystallization conditions, cryoprotectant and diffraction limits of the best crystals are summarized in Table 3.13. The best resolution observed for the Atg5 Atg16L1(1-113) complex with $\operatorname{IP}(1,3)_{2}$ was $7 \AA$. The Atg5 Atg16L1(1-106) crystals with $\operatorname{IP}(1,3)_{2}$ diffracted even weaker, only up to $10 \AA$. 
Table 3.13: Crystallization conditions, cryoprotectants, and diffraction resolution data for the 10 best tested crystals of murine Atg5 Atg16L1(1-106) with IP(1,3) 2 and Atg5 Atg16L1(1-113) with IP(1,3)

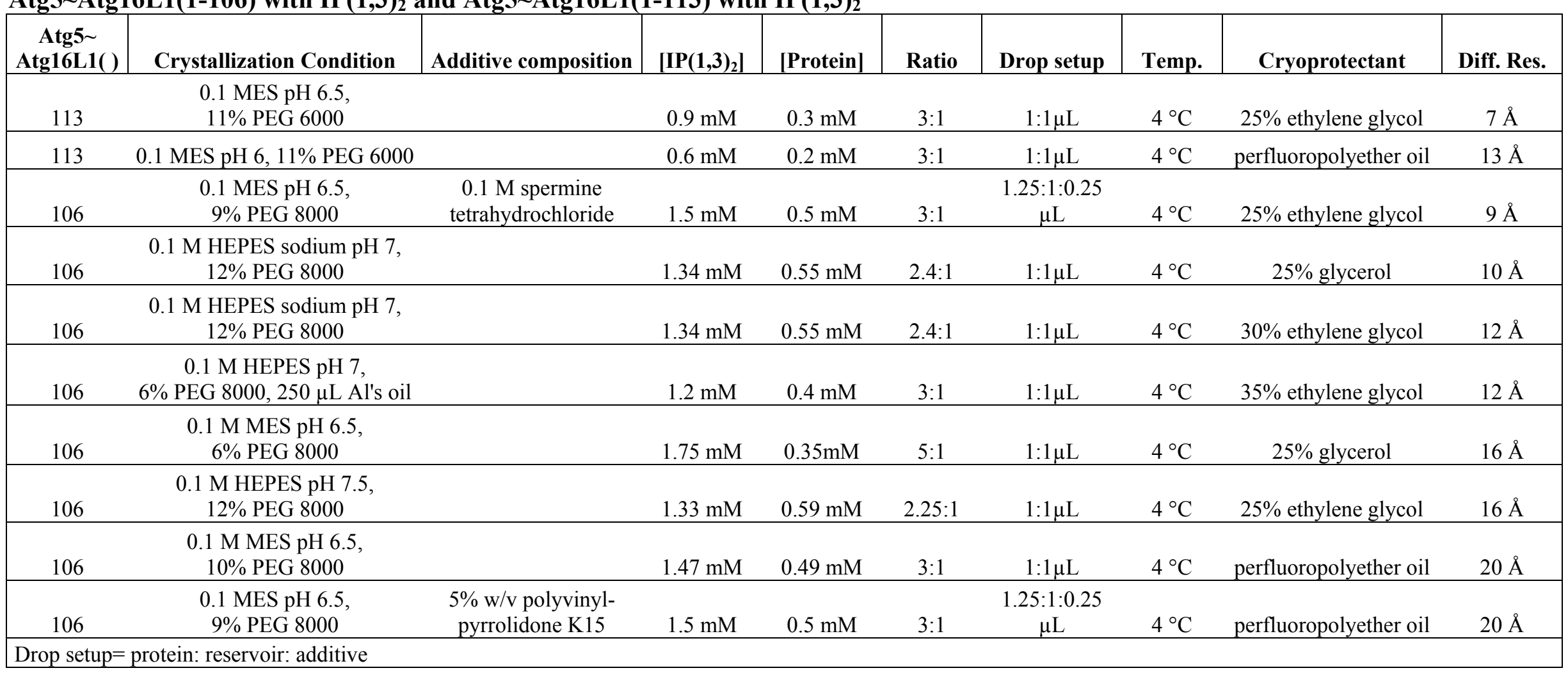




\subsubsection{Crystallization and structure determination of yeast Atg5 Atg16 complexes with inositol diphosphate}

\section{Crystallization of yeast Atg5 Atg16(1-46)}

The structures of the yeast Atg5 Atg16(1-46) and Atg5 Atg16(1-57) complexes were determined at $2.2 \AA$ and $2.0 \AA$, respectively, by Matsushita et al. (2007). Since I could show that the yeast Atg5 Atg16(1-46) and Atg5 Atg16(1-57) complexes also bind phosphoinositides (Fig. 3.44), I tried to reproduce the published crystallization conditions of the Atg5 Atg16(1-46) complex and soak the crystals with IP(1,5)2. Crystals grew at different concentrations of PEG 3350 at $\mathrm{pH} 7.5$ in 24-well Linbro plates using the hanging drop method at $20^{\circ} \mathrm{C}$. The concentration of Atg5 Atg16(1-46) tested ranged from 3 to 28 $\mathrm{mg} / \mathrm{mL}$. Rod-shaped crystals grew within 1 to 2 weeks. The crystallization conditions are summarized in Table 3.14. Pictures of the yeast Atg5 Atg16(1-46) crystals are shown in Figure 3.3.7.A.

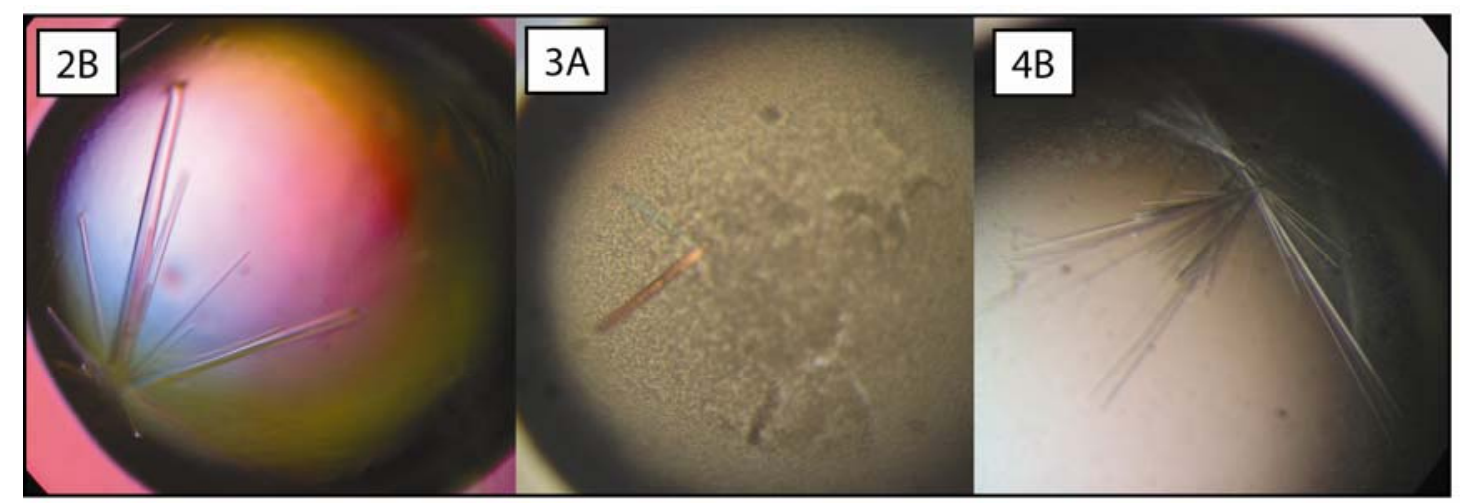

Figure 3.63: Crystals of the yeast Atg5 Atg16(1-46) complex used for soaking with $\operatorname{IP}(1,5)_{2}$ or $\mathrm{Na}_{2} \mathrm{SO}_{4}$. Numbers correspond to the crystallization conditions given in Table 3.14.

Crystals of yeast Atg5 Atg16(1-46) were directly soaked in cryoprotectant containing $\operatorname{IP}(1,5)_{2}$ between 3 and 30 minutes at $20{ }^{\circ} \mathrm{C}$ or $4{ }^{\circ} \mathrm{C}$ before being flash-cooled in liquid nitrogen. Different cryoprotectant combinations were tested: 24\% PEG 3350 and $35 \%$ glycerol with $2 \mathrm{mg} / \mathrm{mL}$ IP( $(1,5)_{2} ; 24 \%$ PEG 3350 and 30\% PEG 400 with 0.44 $\mathrm{mg} / \mathrm{mL} \mathrm{IP}(1,5)_{2} ; 35 \%$ PEG 3350, $15 \%$ PEG 400 with $0.3 \mathrm{mg} / \mathrm{mL} \mathrm{IP}(1,5)_{2}$; and $30 \%$ ethylene glycol with $1 \mathrm{mM} \operatorname{IP}(1,5)_{2}$. A number of crystals were also soaked in $30 \%$ ethylene glycol with $200 \mathrm{mM} \mathrm{Na}_{2} \mathrm{SO}_{4}$. The positions of sulfate ions could help to identify the PIP binding site since they might bind at the sites of the phosphates in the PIP head group. 


\section{Data collection and structure determination of IP(1,5) ${ }_{2}$-soaked yeast Atg5 Atg16(1-46) crystals}

Diffraction data of cryo-cooled crystals were collected at $100 \mathrm{~K}$ on a Pilatus detector at the Swiss Light Source (Switzerland) beamline PXII. Four datasets of the Atg5 Atg16(1-46) complex soaked in IP $(1,5)_{2}$ were collected with resolutions ranging from $3.1 \AA$ up to $2.3 \AA$ as summarized in Table 3.14. Data were processed and scaled using the $X D S$ software package (Kabsch 2010a, b).

Table 3.14: Diffraction of yeast Atg5 Atg16(1-46) crystals soaked with ligand

\begin{tabular}{|c|c|c|c|c|c|c|c|c|}
\hline $\begin{array}{c}\text { Crystallization } \\
\text { condition }\end{array}$ & $\begin{array}{l}\text { Protein } \\
\text { conc. }\end{array}$ & $\begin{array}{l}\text { Drop } \\
\text { setup }\end{array}$ & $\begin{array}{l}\text { Setup } \\
\text { Temp. }\end{array}$ & $\begin{array}{c}\text { Cryo- } \\
\text { protectant }\end{array}$ & $\begin{array}{l}\text { Soaking } \\
\text { ligand }\end{array}$ & $\begin{array}{l}\text { Soak } \\
\text { time }\end{array}$ & $\begin{array}{l}\text { Soak } \\
\text { temp. }\end{array}$ & $\begin{array}{l}\text { Diff. } \\
\text { Res. }\end{array}$ \\
\hline $\begin{array}{c}0.1 \mathrm{M} \text { HEPES pH } \\
7.5,22 \% \text { PEG } 3350\end{array}$ & $5 \mathrm{mg} / \mathrm{mL}$ & $2: 2 \mu \mathrm{L}$ & $4^{\circ} \mathrm{C}$ & $\begin{array}{c}24 \% \text { PEG } 3350, \\
35 \% \text { glycerol }\end{array}$ & $\begin{array}{l}2 \mathrm{mg} / \mathrm{mL} \\
\operatorname{IP}(1,5) 2\end{array}$ & $30 \mathrm{~min}$ & $4{ }^{\circ} \mathrm{C}$ & $2.3 \AA$ \\
\hline $\begin{array}{c}0.1 \mathrm{M} \text { HEPES pH } \\
7.5,22 \% \text { PEG } 3350\end{array}$ & $5 \mathrm{mg} / \mathrm{mL}$ & $2: 2 \mu \mathrm{L}$ & $4^{\circ} \mathrm{C}$ & $\begin{array}{c}24 \% \text { PEG } 3350, \\
35 \% \text { glycerol }\end{array}$ & $\begin{array}{c}2 \mathrm{mg} / \mathrm{mL} \\
\operatorname{IP}(1,5)_{2}\end{array}$ & $30 \mathrm{~min}$ & $20^{\circ} \mathrm{C}$ & $2.8 \AA$ \\
\hline $\begin{array}{c}0.1 \mathrm{M} \text { HEPES pH } \\
7.5,22 \% \text { PEG } 3350\end{array}$ & $5 \mathrm{mg} / \mathrm{mL}$ & $2: 2 \mu \mathrm{L}$ & $4^{\circ} \mathrm{C}$ & $\begin{array}{c}24 \% \text { PEG } 3350, \\
30 \% \text { PEG } 400\end{array}$ & $\begin{array}{c}0.4 \mathrm{mg} / \mathrm{mL} \\
\operatorname{IP}(1,5)_{2}\end{array}$ & $30 \mathrm{~min}$ & $20^{\circ} \mathrm{C}$ & $3.1 \AA$ \\
\hline $\begin{array}{c}0.1 \mathrm{M} \text { HEPES pH } \\
7.5,22 \% \text { PEG } 3350\end{array}$ & $5 \mathrm{mg} / \mathrm{mL}$ & $2: 2 \mu \mathrm{L}$ & $4^{\circ} \mathrm{C}$ & $\begin{array}{c}35 \% \text { PEG } 3350, \\
15 \% \text { PEG } 400\end{array}$ & $\begin{array}{c}0.3 \mathrm{mg} / \mathrm{mL} \\
\mathrm{IP}(1,5)_{2}\end{array}$ & $30 \mathrm{~min}$ & $20^{\circ} \mathrm{C}$ & $3.1 \AA$ \\
\hline $\begin{array}{c}\text { 0.1 M HEPES pH } \\
7.5,11 \% \text { PEG } 3350\end{array}$ & $\begin{array}{c}18.5 \\
\mathrm{mg} / \mathrm{mL} \\
\end{array}$ & $2: 2 \mu \mathrm{L}$ & $4^{\circ} \mathrm{C}$ & $\begin{array}{c}30 \% \text { ethylene } \\
\text { glycol }\end{array}$ & $\begin{array}{r}1 \mathrm{mM} \\
\operatorname{IP}(1,5)_{2}\end{array}$ & $\begin{array}{l}3-5 \\
\min \\
\end{array}$ & $20^{\circ} \mathrm{C}$ & $4.5 \AA$ \\
\hline $\begin{array}{c}\text { 0.1 M HEPES pH } \\
7.5,12 \% \text { PEG } 3350\end{array}$ & $\begin{array}{c}20 \\
\mathrm{mg} / \mathrm{mL}\end{array}$ & $2: 2 \mu \mathrm{L}$ & $4^{\circ} \mathrm{C}$ & $\begin{array}{c}30 \% \text { ethylene } \\
\text { glycol }\end{array}$ & $\begin{array}{r}1 \mathrm{mM} \\
\operatorname{IP}(1,5)_{2}\end{array}$ & $\begin{array}{l}3-5 \\
\min \end{array}$ & $20^{\circ} \mathrm{C}$ & $8 \AA$ \\
\hline $\begin{array}{c}\text { 0.1 M HEPES pH } \\
7.5,14 \% \text { PEG } 3350\end{array}$ & $5 \mathrm{mg} / \mathrm{mL}$ & $2: 2 \mu \mathrm{L}$ & $4{ }^{\circ} \mathrm{C}$ & $\begin{array}{c}30 \% \text { ethylene } \\
\text { glycol }\end{array}$ & $\begin{array}{l}200 \mathrm{mM} \\
\mathrm{Na}_{2} \mathrm{SO}_{4} \\
\end{array}$ & $\begin{array}{l}3-5 \\
\min \\
\end{array}$ & $20^{\circ} \mathrm{C}$ & $\begin{array}{l}\text { No } \\
\text { diff. }\end{array}$ \\
\hline $\begin{array}{c}\text { 0.1 M HEPES pH } \\
7.5,11 \% \text { PEG } 3350\end{array}$ & $\begin{array}{c}18.5 \\
\mathrm{mg} / \mathrm{mL}\end{array}$ & $2: 2 \mu \mathrm{L}$ & $4^{\circ} \mathrm{C}$ & $\begin{array}{c}30 \% \text { ethylene } \\
\text { glycol }\end{array}$ & $\begin{array}{l}200 \mathrm{mM} \\
\mathrm{Na}_{2} \mathrm{SO}_{4}\end{array}$ & $\begin{array}{l}3-5 \\
\min \end{array}$ & $20^{\circ} \mathrm{C}$ & $\begin{array}{l}\text { No } \\
\text { diffr. }\end{array}$ \\
\hline $\begin{array}{c}0.1 \text { M HEPES pH } \\
7.5,14 \% \text { PEG } 3350\end{array}$ & $5 \mathrm{mg} / \mathrm{mL}$ & $2: 2 \mu \mathrm{L}$ & $4^{\circ} \mathrm{C}$ & $\begin{array}{c}30 \% \text { ethylene } \\
\text { glycol }\end{array}$ & $\begin{array}{l}200 \mathrm{mM} \\
\mathrm{Na}_{2} \mathrm{SO}_{4}\end{array}$ & $\begin{array}{l}3-5 \\
\min \end{array}$ & $20^{\circ} \mathrm{C}$ & $\begin{array}{l}\text { No } \\
\text { diffr. }\end{array}$ \\
\hline
\end{tabular}

The crystal belonged to the monoclinic space group $\mathrm{P} 2{ }_{1}$. The unit-cell parameters of the best diffracting crystal are $a=56.9 \AA, b=98.0 \AA, c=66.3 \AA, \alpha=\gamma=90.0^{\circ}, \beta=108.6^{\circ}$. The data collection statistics are found in Table 3.15. Programs supported by the CCP4 software suite were used for further analysis (Collaborative Computational Project Number 4 1994, Potterton 2003). The Matthews coefficient (Matthews 1968, 1976) indicated that there were two molecules of Atg5 Atg16 per asymmetric unit (Fig. 3.64 panel A). The program MOLREP (Vagin 1997) was then used for molecular replacement using the Atg5 Atg16(1-46) structure (PDB ID: 2DYM) (Matsushita 2007) as a search model. REFMAC5 (Murshudov 1997) was used for subsequent rigid body and restrained 
refinement and calculation of the electron density maps. The R-factor after restrained refinement was $26.0 \%$ and the free R-factor was $31.0 \%$.

Table 3.15: Data statistics for yeast Atg5 Atg16(1-46) soaked with IP(1,5)2

\begin{tabular}{|c|c|}
\hline & $\operatorname{Atg5} \sim \operatorname{Atg} 16(1-46)+\operatorname{IP}(1,5)_{2}$ \\
\hline Space group & \multirow{4}{*}{$\begin{array}{c}\text { P } 2_{1} \\
56.9,98.0,66.3\left(\beta=108.6^{\circ}\right) \\
\text { SLS PXII } \\
1.0 \AA\end{array}$} \\
\hline Unit cell dimensions $a, b, c,(\AA)$ & \\
\hline Beamline & \\
\hline Wavelength & \\
\hline Resolution of data ( $(\AA)$ (high-resolution bin) & $50-2.3(2.3-2.4)$ \\
\hline No. of observations/ no. unique reflections & $105607 / 30498$ \\
\hline Completeness (\%), total (high) & $99.2(99.2)$ \\
\hline$I / \sigma(I)$, total (high) & $14.6(3.6)$ \\
\hline R-factor observed (\%), total (high) & $6.6(40.5)$ \\
\hline Wilson $B$ factor $\left(\AA^{2}\right)$ & 40.5 \\
\hline
\end{tabular}

$R$-factor $=\Sigma_{\mathrm{hkl}} \Sigma_{i}\left|I_{i}(\mathrm{hkl})-\langle I(\mathrm{hkl})\rangle\right| / \Sigma_{\mathrm{hkl}} \Sigma_{i}\left|I_{i}(\mathrm{hkl})\right| ;$ for $n$ independent reflections and $i$ observations of a given reflection, where $\langle I(\mathrm{hkl})\rangle=$ average intensity of the $i$ observations.

The graphics program COOT (Emsley 2004, 2010) was used to visualize the structure and electron density maps. The backbone and also the side chains were clearly resolved (Fig. 3.64 panels B and C). However, no electron density was observed for the first 22 amino acid residues of Atg16 as they were disordered. Furthermore, no positive peaks in the difference electron density maps which could correspond to the $\operatorname{IP}(1,5)_{2}$ ligand were found for either pair of Atg5 Atg16 chains (Fig. 3.64 panels D and E). All collected datasets were processed but none of them contained a bound $\operatorname{IP}(1,5)_{2}$ molecule. 


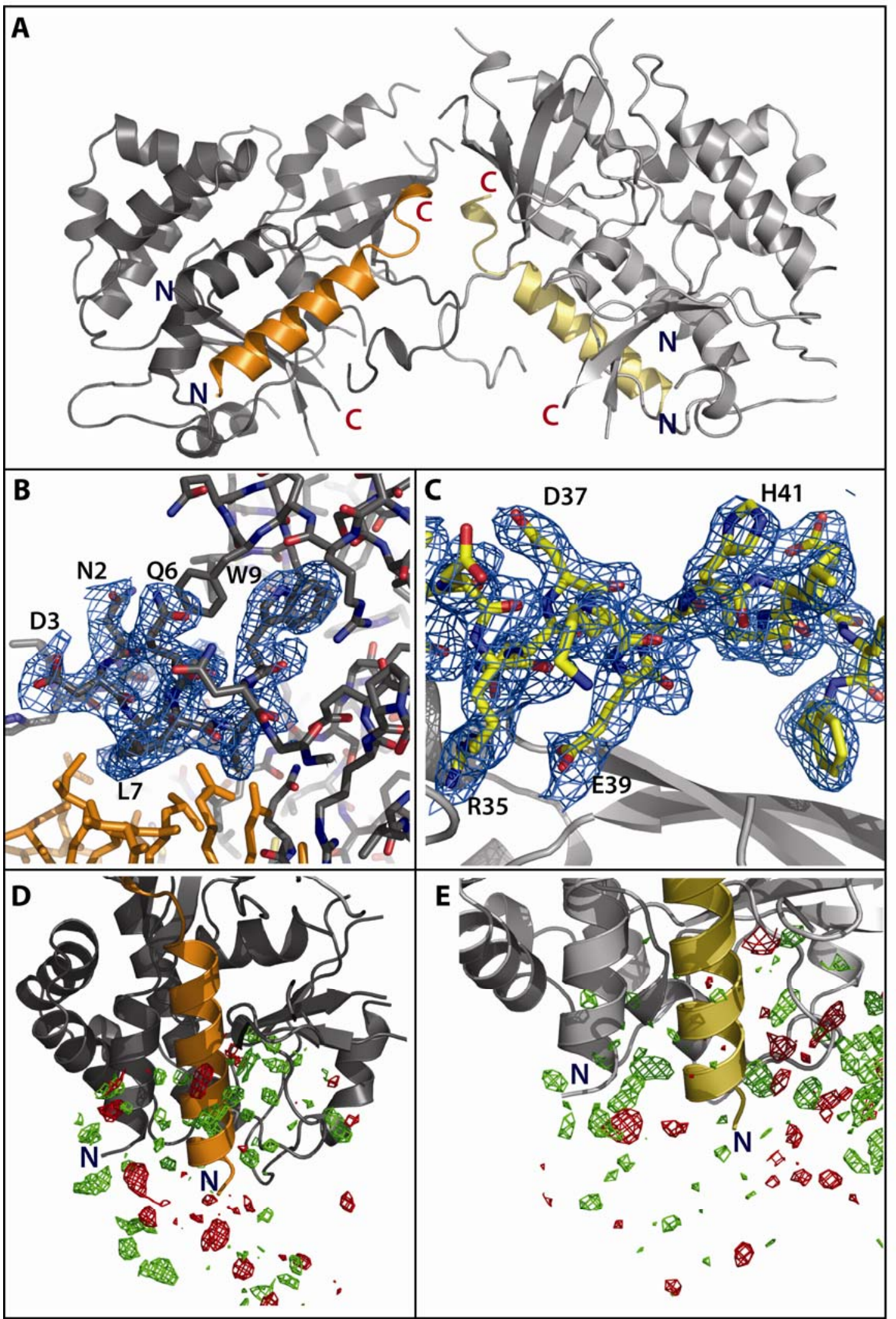

Fig. 3.64: Structure of the yeast Atg5 Atg16(1-46) complex soaked in IP(1,5) $)_{2}$ (A) Cartoon representation of the two molecules of Atg5 Atg16 present in the asymmetric unit. The $2.3 \AA$ resolution $2 \mathrm{mF}_{\mathrm{o}}-\mathrm{DF}_{\mathrm{c}}$ electron density map around (B) Atg5 chain A, contoured at $1 \sigma$ and (C) Atg16 chain $\mathrm{D}$, contoured at $1 \sigma . \mathrm{mF}_{\mathrm{o}}-\mathrm{DF}_{\mathrm{c}}$ difference electron density maps showing positive (green) and negative (red) density, contoured at $\pm 2.5 \sigma$ for Atg5 Atg16 chains (D) A \& B and (E) C \& D. N and C indicate the termini. Figures were prepared with PYMOL (http://pymol.org). 


\section{Crystallization of yeast Atg5 Atg16(1-57)}

I also tried to co-crystallize and soak yeast Atg5 Atg16(1-57) crystals with $\operatorname{IP}(1,3)_{2}$. Crystallization screening in 96-well MRC sitting drop plates was performed using IP $(1,3)_{2}$ which was directly reconstituted with a solution of the Atg5 Atg16(1-57) complex to achieve the highest ligand concentration possible (approx. $2.5 \mathrm{mM}$ ). The following commercial screening suites from Qiagen were utilized: Classics, Classics Lite, Protein Complex, PEGs, PEGsII, and Cryos. The plates were set up and stored at $4{ }^{\circ} \mathrm{C}$.

A condition comprised of 15\% (w/w) PEG 6000 and 5\% (v/v) glycerol yielded crystals. Crystallization conditions were optimized using 24-well Linbro plates with the hanging drop method. The concentration of PEG 6000 was varied from 6 to $25 \%$. The second precipitant component was either glycerol, which was tested in the range of 5 to $40 \%$ or ethylene glycol. Here the concentration was varied between 20 and $35 \%$. The protein concentration was tested from 1 to $20 \mathrm{mg} / \mathrm{mL}(0.02 \mathrm{mM}$ to $0.48 \mathrm{mM})$. The concentration of $\operatorname{IP}(1,3)_{2}$ ranged from 1.3 to $2.6 \mathrm{mM}$ with the ratio of $\operatorname{IP}(1,3)_{2}$ :protein being as high as was attainable at that protein concentration. To add another variable, in some cases the IP(1,3) 2 was adjusted to $20 \mathrm{mM}$ Tris $\mathrm{pH} 7.4$ and $200 \mathrm{mM} \mathrm{NaCl}$ before being added to the Atg5 Atg16(1-57) complex. Crystals grew very quickly, usually within one to two days. The crystallization conditions for the best diffracting crystals are summarized in Table 3.16 and crystal pictures are shown in Figure 3.65.

As glycerol was already a component of the precipitant, it was endeavored to increase its concentration to the point that the mother liquor could serve as a cryoprotectant. In such a case, the crystals can be transferred directly from the crystallization drop into liquid nitrogen. Some crystals were also slowly streaked in perfluoropolyether oil to provide a cryoprotective coating before flash cooling in liquid nitrogen. For some crystals whose concentration of glycerol included in the precipitant was too low for the formation of vitreous glass, an additional soaking step directly in cryoprotectant solution containing the ligand was performed. The amount of time the crystals were grown before being harvested was an additional variable tested in the event that $\operatorname{IP}(1,3)_{2}$ hydrolysis and dissociation from the complex occurred rapidly despite being kept at $4{ }^{\circ} \mathrm{C}$. 
Table 3.16: Crystallization conditions, cryoprotectants, and diffraction resolution data for the best crystals of yeast Atg5 Atg16(1-57) with $\operatorname{IP}(1,3)_{2}$

\begin{tabular}{|c|c|c|c|c|c|c|c|c|c|c|c|}
\hline & Crystallization Condition & $\begin{array}{c}{\left[\mathrm{IP}(1,3)_{2}\right]} \\
\mathrm{mM}\end{array}$ & $\begin{array}{c}\text { [Protein] } \\
\text { mM }\end{array}$ & $\begin{array}{l}\text { Molar } \\
\text { ratio }\end{array}$ & $\begin{array}{l}\text { Drop } \\
\text { setup }\end{array}$ & Temp. & Soaking solution & $\begin{array}{l}\text { Soak } \\
\text { time }\end{array}$ & $\begin{array}{l}\text { Growth } \\
\text { before } \\
\text { harvest }\end{array}$ & Cryoprotectant & $\begin{array}{l}\text { Diff. } \\
\text { Res. }\end{array}$ \\
\hline 1 & $15 \%$ PEG $6000,25 \%$ glycerol & $2.0,2.3 *$ & $0.24,0.12$ & $8: 1,19: 1$ & $2: 2 \mu \mathrm{L}$ & $4^{\circ} \mathrm{C}$ & & & 2 weeks & & $1.8 \AA$ \\
\hline 2 & $\begin{array}{l}15 \% \text { PEG } 6000, \quad 35 \% \\
\text { ethylene glycol }\end{array}$ & $2.0 *$ & 0.24 & $8: 1$ & $1: 1 \mu \mathrm{L}$ & $4^{\circ} \mathrm{C}$ & & & 3-4 days & & $1.8 \AA$ \\
\hline 3 & $\begin{array}{c}15 \% \text { PEG } 6000, \\
35 \% \text { ethylene glycol }\end{array}$ & $2.0 *$ & 0.24 & $8: 1$ & $1: 1 \mu \mathrm{L}$ & $4{ }^{\circ} \mathrm{C}$ & & & 3-4 days & perfluoropolyether oil & $1.8 \AA$ \\
\hline 4 & $17 \%$ PEG $6000,5 \%$ glycerol & $2.0,2.3 *$ & $0.24,0.12$ & $8: 1,19: 1$ & $1: 1 \mu \mathrm{L}$ & $4^{\circ} \mathrm{C}$ & & & 1 month & perfluoropolyether oil & $2.0 \AA$ \\
\hline 5 & $15 \%$ PEG $6000,10 \%$ glycerol & $2.0 *$ & 0.24 & $8: 1$ & $1: 1 \mu \mathrm{L}$ & $4^{\circ} \mathrm{C}$ & $\begin{array}{c}1.2 \mathrm{mM} \mathrm{IP}(1,3)_{2}, 15 \% \\
\text { PEG } 6000,10 \% \\
\text { glycerol, } 15 \% \text { PEG } 400\end{array}$ & 2.5 weeks & 1 month & & $2.0 \AA$ \\
\hline 6 & $15 \%$ PEG $6000,10 \%$ glycerol & $2.0 *$ & 0.24 & $8: 1$ & $1: 1 \mu \mathrm{L}$ & $4^{\circ} \mathrm{C}$ & $\begin{array}{c}1.2 \mathrm{mM} \mathrm{IP}(1,3)_{2}, 15 \% \\
\text { PEG } 6000,10 \% \\
\text { glycerol, } 15 \% \text { PEG } 400\end{array}$ & 2.5 weeks & 1 month & perfluoropolyether oil & $2.0 \AA$ \\
\hline 7 & $17 \%$ PEG $6000,10 \%$ glycerol & $2.0 *$ & 0.24 & $8: 1$ & $1: 1 \mu \mathrm{L}$ & $4{ }^{\circ} \mathrm{C}$ & & & 2 weeks & perfluoropolyether oil & $2.0 \AA$ \\
\hline 8 & $15 \%$ PEG $6000,15 \%$ glycerol & $2.0,2.3 *$ & $0.24,0.12$ & $8: 1,19: 1$ & $1: 1 \mu \mathrm{L}$ & $4^{\circ} \mathrm{C}$ & & & 2 weeks & perfluoropolyether oil & $2.0 \AA$ \\
\hline 9 & $17 \%$ PEG $6000,15 \%$ glycerol & $2.3 *$ & 0.12 & $19: 1$ & $1: 1 \mu \mathrm{L}$ & $4^{\circ} \mathrm{C}$ & & & 1 month & perfluoropolyether oil & $2.0 \AA$ \\
\hline 10 & $15 \%$ PEG $6000,20 \%$ glycerol & $2.3 *$ & 0.12 & $19: 1$ & $1: 1 \mu \mathrm{L}$ & $4{ }^{\circ} \mathrm{C}$ & & & 1 month & perfluoropolyether oil & $2.0 \AA$ \\
\hline 11 & $15 \%$ PEG $6000,25 \%$ glycerol & $2.3 *$ & 0.12 & $19: 1$ & $1: 1 \mu \mathrm{L}$ & $4^{\circ} \mathrm{C}$ & & & 2 weeks & perfluoropolyether oil & $2.0 \AA$ \\
\hline 12 & $15 \%$ PEG $6000,30 \%$ glycerol & $2.3 *$ & 0.12 & $19: 1$ & $1: 1 \mu \mathrm{L}$ & $4^{\circ} \mathrm{C}$ & & & 1 week & & $2.0 \AA$ \\
\hline 13 & $17 \%$ PEG $6000,30 \%$ glycerol & $2.3 *$ & 0.12 & $19: 1$ & $1: 1 \mu \mathrm{L}$ & $4{ }^{\circ} \mathrm{C}$ & & & 1 week & & $2.0 \AA$ \\
\hline 14 & $\begin{array}{c}15 \% \text { PEG } 6000, \\
20 \% \text { ethylene glycol }\end{array}$ & $2.4 *$ & 0.06 & $40: 1$ & $1: 1 \mu \mathrm{L}$ & $4^{\circ} \mathrm{C}$ & & & 1 week & & $2.0 \AA$ \\
\hline 15 & $\begin{array}{c}15 \% \text { PEG } 6000, \\
25 \% \text { ethylene glycol }\end{array}$ & $2.4 *$ & 0.06 & $40: 1$ & $1: 1 \mu \mathrm{L}$ & $4{ }^{\circ} \mathrm{C}$ & & & 1 week & & $2.2 \AA$ \\
\hline 16 & $15 \%$ PEG $6000,30 \%$ glycerol & $2.3 *$ & 0.12 & $19: 1$ & $1: 1 \mu \mathrm{L}$ & $4{ }^{\circ} \mathrm{C}$ & & & 1 week & perfluoropolyether oil & $2.3 \AA$ \\
\hline
\end{tabular}




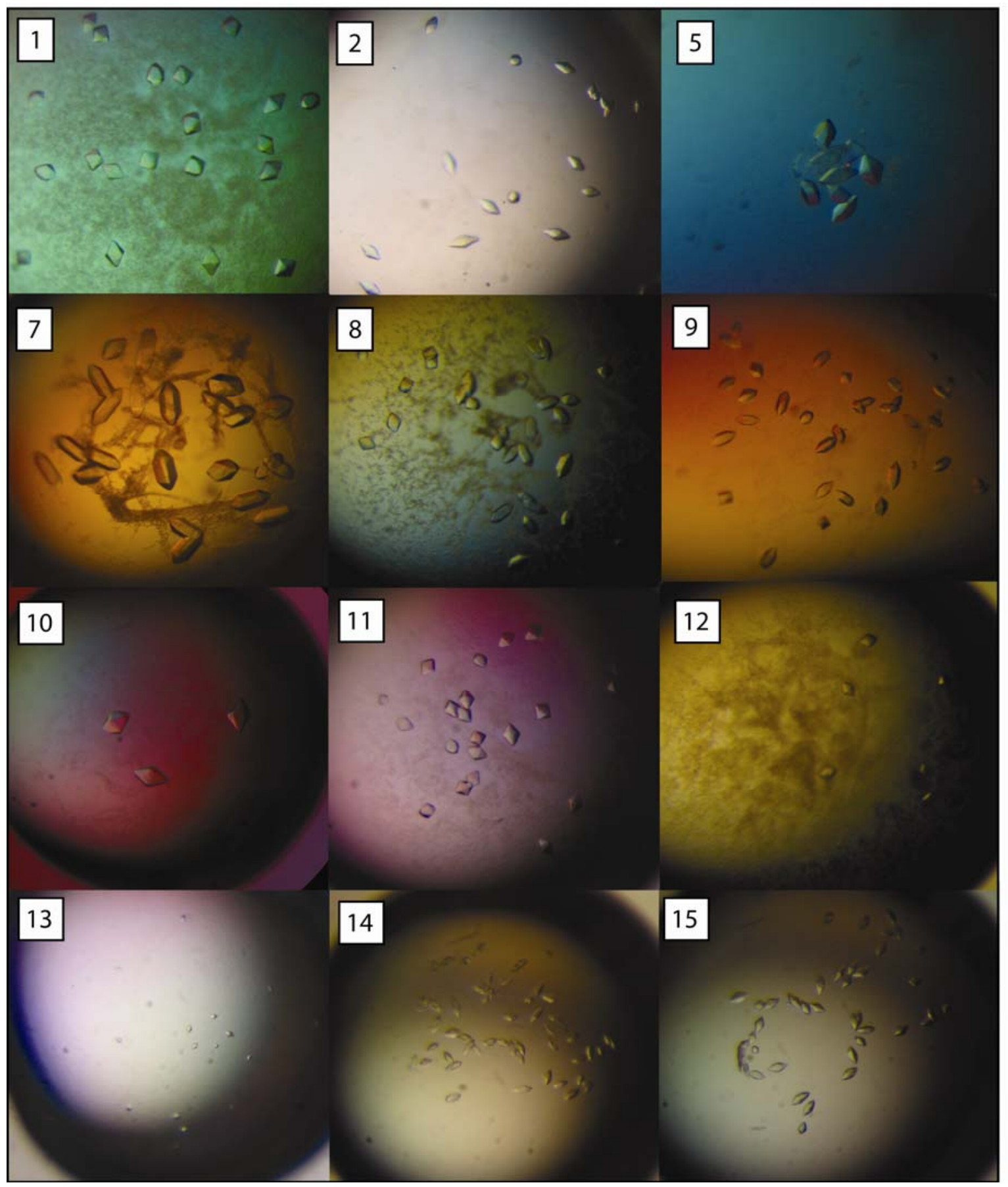

Figure 3.65: Crystals of the yeast Atg5 Atg16(1-57) complex crystallized in the presence of IP(1,3) 2. Numbers correspond to the crystallization conditions in Table 3.16. Crystals typically grew within 1 to 2 days. Crystals in number 6 were soaked in cryoprotectant containing $\operatorname{IP}(1,3)_{2}$ for 2.5 weeks. Scale bar $=90 \mu \mathrm{m}$. 
Data collection and structure determination of soaked and co-crystallized yeast Atg5 Atg16(1-57) crystals

Diffraction data of cryo-cooled crystals were collected at $100 \mathrm{~K}$ on a Pilatus detector at the Swiss Light Source (Switzerland) beamline PXII. The cryoprotectant conditions and diffraction resolution are summarized in Table 3.16. The datasets collected from Atg5 Atg16(1-57) + IP(1,3) $)_{2}$ crystals sampled a wide range of crystallization conditions, cryoprotectants, soaking times, and amount of time grown before being flashcooled. The collected datasets ranged in their maximum resolution between $1.8 \AA$ and 2.3 A. Data were processed and scaled using the $X D S$ software package (Kabsch 2010a, b). The crystals belonged to the tetragonal space group P42. The data collection statistics for a dataset diffracting to $1.8 \AA$ resolution is summarized in Table 3.17. The unit cell dimensions are $a=73.6 \AA, b=73.6 \AA, c=148.5 \AA, \alpha=\beta=\gamma=90.0^{\circ}$ for this crystal.

Table 3.17: Data statistics for yeast Atg5 Atg16(1-57) co-crystallized with $\operatorname{IP}(1,3)_{2}$

\begin{tabular}{|c|c|}
\hline & $\operatorname{Atg} 5 \sim \operatorname{Atg} 16(1-57)+\operatorname{IP}(1,3)_{2}$ \\
\hline Space group & P4 $22_{1} 2$ \\
\hline Unit cell dimensions $a, b, c,(\AA)$ & $73.6,73.6,149.0$ \\
\hline Beamline & SLS PXII \\
\hline Wavelength & $1.0 \AA$ \\
\hline Resolution of data ( $(\AA)$ (high-resolution bin) & $50-1.8(1.8-1.85)$ \\
\hline No. of observations/ no. unique reflections & $250093 / 38752$ \\
\hline Completeness (\%), total (high) & $99.9(100)$ \\
\hline$I / \sigma(I)$, total (high) & $28.0(5.4)$ \\
\hline R-factor observed (\%), total (high) & $3.8(33.2)$ \\
\hline Wilson $B$ factor $\left(\AA^{2}\right)$ & 32.3 \\
\hline
\end{tabular}

$R$-factor $=\Sigma_{\mathrm{hk} \mid} \Sigma_{i}\left|I_{i}(\mathrm{hkl})-\langle I(\mathrm{hkl})\rangle\right| / \Sigma_{\mathrm{hk} 1} \Sigma_{i}\left|I_{i}(\mathrm{hkl})\right| ; \quad$ for $n$ independent reflections and $i$ observations of a given reflection, where $\langle I(\mathrm{hkl})\rangle=$ average intensity of the $i$ observations.

Programs of the $C C P 4$ software suite were used for structure determination (Collaborative Computational Project Number 4 1994, Potterton 2003). The Matthews coefficient (Matthews 1968, 1976) indicated that there was one molecule of the Atg5 Atg16 complex per asymmetric unit (Figure 3.66 panel F). The program MOLREP (Vagin 1997) was used for molecular replacement using the Atg5 Atg16(1-57) structure as a search model (Matsushita 2007) (PDB ID: 2DYO). REFMAC5 (Murshudov 1997) was used for rigid body and restrained refinement and calculation of the electron density maps. 
The electron density maps were of good quality. Regions of Atg5 (Fig. 3.66 panel A) and Atg16 (Fig. 3.66 panel B) are shown as examples. However, the first 21 amino acid residues of Atg16 were also disordered like in the published structures (Matsushita 2007). There was positive density in the difference electron density map at the N-terminus of Atg5 (Fig. 3.66 panel C). Five residues preceding the N-terminal methionine that acted as a linker between Atg5 and the His-tag were built in and the structure was refined with REFMAC5 (Murshudov 1997) giving an R-factor of $24.9 \%$ and a free $\mathrm{R}$-factor of $27.1 \%$ and is shown in Figure 3.66 panel D. The difference electron density map was analyzed, but no positive density fitting the $\operatorname{IP}(1,3)_{2}$ ligand was located. The structures were determined for all data sets collected, sampling different crystallization conditions, ligand soaking times, and length of growth before harvest. However, there is no evidence for bound $\operatorname{IP}(1,3)_{2}$.

The structures of yeast Atg5 Atg16(1-46) and Atg5 Atg16(1-57) deposited in the PDB were analyzed to check whether any of the N-terminal Atg16 residues lacking in the models might have been missed by the authors (Matsushita 2007). The structure factors for both structures structures (PDB ID: 2DYM, 2DYO) were downloaded and the electron density maps were calculated. The difference electron density maps were closely scrutinized for any unaccounted for density near the N-terminus of Atg16 but the Nterminal Atg16 residues are indeed disordered. 


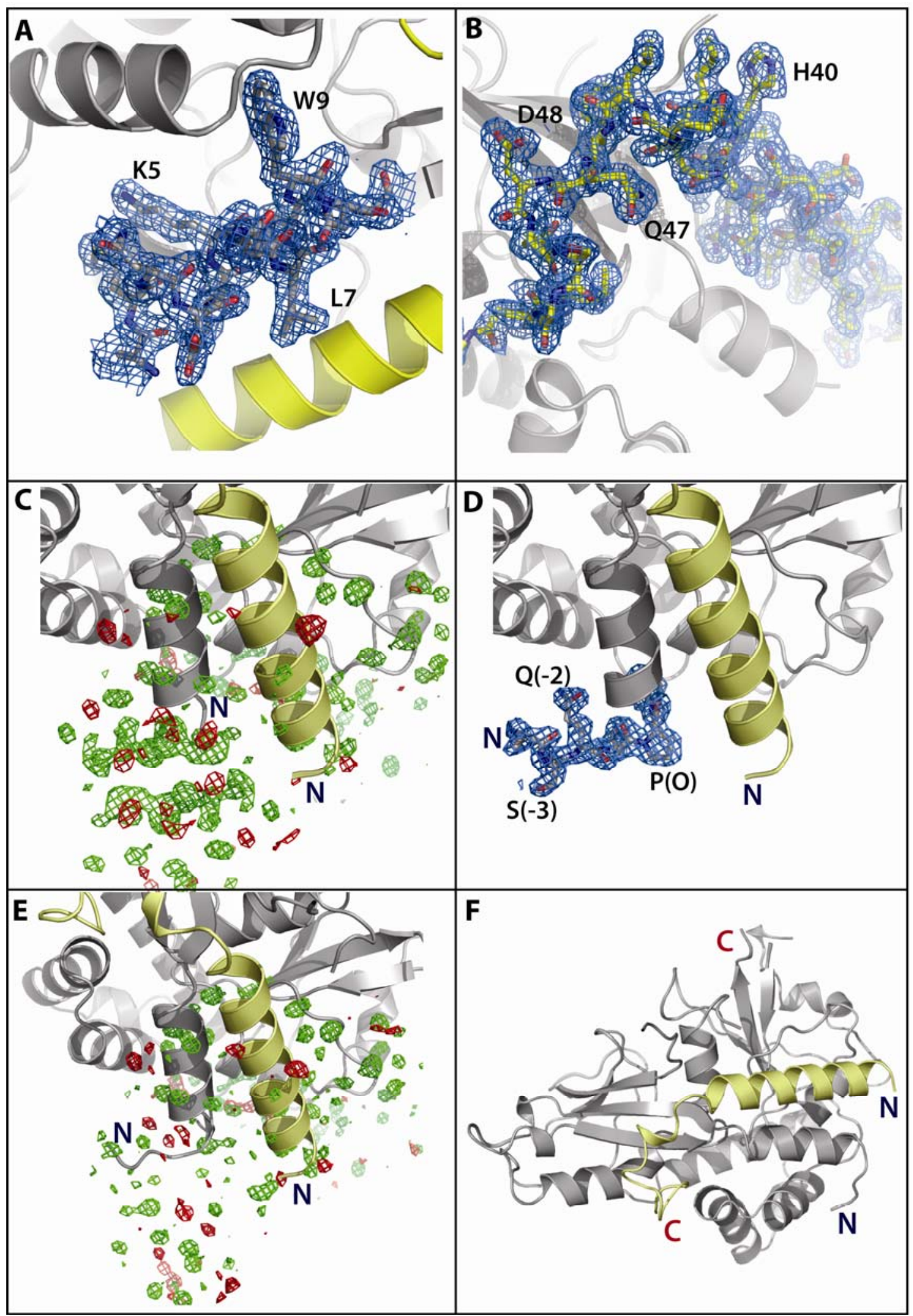

Fig. 3.66: Structure of the yeast Atg5 Atg16(1-57) complex crystallized in the presence of IP(1,3) The $1.8 \AA$ resolution $2 \mathrm{mF}_{\mathrm{o}}-\mathrm{DF}_{\mathrm{c}}$ electron density map, contoured at $1 \sigma$, around (A) Atg5 and (B) Atg16. (C) $\mathrm{mF}_{\mathrm{o}}-\mathrm{DF}_{\mathrm{c}}$ difference electron density map showing positive (green) and negative (red) density contoured at $\pm 2.5 \sigma$. (D) $2 \mathrm{mF}_{\mathrm{o}}-\mathrm{DF}_{\mathrm{c}}$ electron density map, contoured at $1 \sigma$, around the five built in linker residues of Atg5 that precede residue $M 1$. (E) $\mathrm{mF}_{\mathrm{o}}-\mathrm{DF}_{\mathrm{c}}$ electron density difference map contoured at \pm 2.50 after Atg5 N-terminal residues were built in and the structure was refined. (F) Cartoon representation of the single molecule of Atg5 Atg16 found in the asymmetric unit. $\mathrm{N}$ and $\mathrm{C}$ indicate the termini.

Figures were prepared with PYMOL (http://pymol.org). 


\section{DISCUSSION}

\subsection{Characterization of mammalian Atg5 Atg16L1 complexes}

\subsubsection{Oligomerization state determination of mammalian Atg5 Atg16L1 complexes}

As oligomerization of the Atg12-Atg5 Atg16 complex via the coiled coil domain of Atg16 is required for autophagy, the elucidation of complex multimerization would be an important contribution to understanding how the complex carries out its essential function (Mizushima 1999, Kuma 2002). One goal of this study was to accurately determine the molecular weight and stoichiometry of the mammalian Atg5 Atg16L1 complex. In earlier studies, the yeast complex was determined to be a $\sim 350 \mathrm{kDa}$ tetrameric complex (Kuma 2002). Mizushima et al. (2003) observed two peaks of the multimerized murine Atg12-Atg5 Atg16L1 complex. The major and minor peaks corresponded to an $\sim 800 \mathrm{kDa}$ octamer and a $\sim 400 \mathrm{kDa}$ tetramer, respectively. The molecular mass in each of these studies was determined by the elution volume from a gel filtration column.

However, the elution volume of a protein during gel filtration analysis depends strongly on the shape of a protein (Stokes radius) (Siegel 1966). A protein with an elongated shape, such as that with a coiled coil domain, will have an overestimated molecular mass when this value is derived from its gel filtration elution volume. The apparently higher molecular weights were also observed when I analyzed the Atg5 Atg16L1 complexes on a gel filtration column. The molecular weights calculated from the elution volumes would correspond to trimers for shorter lengths of the Atg16L1 coiled coil domain up to heptamers for the full coiled coil domain (residues 1-265) of Atg16L1.

To eliminate inaccuracies in molecular weight determination due to the nonglobular shape of Atg5 Atg16L1, multiple angle laser light scattering (MALLS) was utilized which avoids bias based on a non-uniform shape. Using this method, the determined molecular weights of the multimerized mammalian Atg5 Atg16L1 complexes correspond to two copies each of Atg5 and Atg16L1. The molecular mass of Atg16L1(53168 ) on its own was also consistent with a dimer. Further support comes from analysis of the diffraction data collected to $5 \AA$ resolution of the mammalian Atg5 Atg16L1(1-106) complex which indicated the presence of three dimers in the asymmetric unit. 
While this study was underway, Fujita et al. (2009) also conducted investigations into the oligomerization state of the murine Atg12-Atg5 Atg16L1 complex. They utilized sucrose density gradient centrifugation and showed that the complex is a dimer. This is in agreement with our findings. Our findings were further confirmed by Fujioka et al. (2010) who determined the structure of the coiled coil domain of yeast $\operatorname{Atg} 16$, which forms a parallel coiled coil dimer.

\subsubsection{Cleavage of Atg16L1 by the calpain protease}

Yousefi et al. (2006) observed that cleavage of Atg5 by calpain-1 led to a $\sim 24 \mathrm{kDa}$ truncated product (residues 1-193) that mediates cell death by triggering caspase activation upon induction by apoptotic stimuli. It was attempted in this study to cleave human Atg5, when in complex with murine Atg16L1, with calpain-1 and calpain-2. However in contrast to the observations of Yousefi et al. (2006), I did not observe cleavage of Atg5. Williams et al. (2008) reported that the activation of calpain did not reduce the amount of full-length Atg5 or produce any cleavage products, which is in agreement with our data. Instead of cleavage of Atg 5 by calpain, I showed for the first time that both calpain-1 and calpain-2 cleave Atg16L1. Cleavage of Atg16L1 was calpain specific as it was inhibited by the addition of the calpain-specific inhibitors calpastatin, ALLN, and ALLM. There are at least two cleavage sites in Atg16L1, one in the N-terminal Atg5-binding domain and one in the coiled coil domain.

A recent study conducted by Norman et al. (2010) revealed that cell death proteases, including calpain, cleave many of the autophagy proteins including some of those involved in the Ubl conjugation system to which Atg5 and Atg16L1 belong. Cleavage by calpain-1 in vitro was observed for Atg5, Atg12, Atg7, Atg10, Atg3, Atg4AD, GABARAP, Atg9, and Beclin-1. Atg7 and Atg3 were also cleaved by caspases. Cleavage of the autophagy proteins involved in the Ubl conjugation systems would abrogate autophagy at the autophagosome formation step and could provide a form of regulation for autophagosome formation.

A regulatory role for calpain in autophagy has already been observed, however it is far from being completely understood. A screen for autophagy inducing and suppressing small molecule compounds revealed that calpains cleave the $\alpha$-subunit of trimeric $G$ proteins $\left(G_{s \alpha}\right)$ (Williams 2008). The activated $G_{s \alpha}$ produces more cAMP which is inhibitory to autophagy. Levels of cAMP affect the Epac-PLC- $-\mathrm{IP}_{3}$ pathway which signals the release of calcium from stores in the ER into the cytosol. Increased cytosolic 
$\mathrm{Ca}^{2+}$ negatively regulates autophagy and compounds which block $\mathrm{Ca}^{2+}$ channels induce autophagy (Gordon 1993, Zhang 2007, Williams 2008). Calpain activity, which occurs through increased intracellular calcium, has been conversely shown to stimulate autophagy in apoptotic cells (Demarchi 2006, Cheng 2008). Xia et al. (2010) have concluded that calpain-1 inhibition prevented cleavage of Atg5 and the Atg5-Atg12 conjugate in cells under normal nutritional conditions (Xia 2010).

Based on the calpain cleavage assays presented here as well as those mentioned above, the cleavage of Atg5 and Atg16L1 by calpain could provide a significant regulatory step for basal autophagy, apoptosis-induced autophagy, and the inhibition of autophagy through the $\mathrm{G}_{\mathrm{s} \alpha}$-cAMP-Epac-PLC- $\varepsilon-\mathrm{IP}_{3}$ mTOR-independent pathway at the stage of autophagosome formation.

\subsection{Characterization of mammalian Rab33B Atg16L1 complexes}

Itoh et al. (2008) discovered that the small GTPase Rab33B interacts with the Atg12-Atg5 Atg16L1 complex through a direct interaction with the coiled coil domain of Atg16L1 (residues 141-265). They also observed that this interaction was GTPdependent, thus making Atg16L1 an effector protein of Rab33B. In our study we confirmed association of the GTPase-deficient truncated Rab33B(30-202)Q92L with the Atg5 Atg16L1(1-265) complex via co-elution of the proteins during analytical gel filtration and an exothermic titration curve observed during isothermal titration calorimetry experiments.

We observed that the interaction between Rab33B(30-202)Q92L and the Atg5 Atg16L1(1-265) complex is of intermediate to strong affinity, approximately in the range of $150 \mathrm{nM}$ to $450 \mathrm{nM}$. The stoichiometry of Rab33B to the Atg16L1 Atg5 complex was determined to be $1: 1$.

I tried to crystallize Rab33B(30-202)Q92L Atg16L1 complexes comprising various portions of the Rab33B-binding domain. Despite the fact that both untagged Atg16L1(172-265) and Atg16L1(172-234) co-eluted from a Ni-sepharose column with His-Rab33B(30-202)Q92L, neither of these complexes crystallized. Only the complex comprising the full Rab33B-binding domain of Atg16L1 (residues 141-265) crystallized. This indicates that the N-terminal portion of the Rab33B-binding domain (residues 141171) of Atg16L1 is important for binding. In fact, limited proteolysis of the Rab33B(30202) Q92L Atg16L1(141-265) yielded a cleavage product of Atg16L1 whose N-terminus 
was intact as determined by $\mathrm{N}$-terminal sequencing and which ended approximately at residue 204. However, untagged Atg16L1(125-204) did not co-elute with HisRab33B(30-202) Q92L. This is in agreement with the observations of the Fukuda lab, where the N-terminal portion of the coiled coil domain of Atg16L1 (residues 80-200) did not bind Rab33B (Itoh 2008).

I also observed that residues 203-265 of Atg16L1 were insufficient for complex formation with Rab33B(30-202)Q92L. In contrast, the Rab33B(30202)Q92L Atg16L1(141-214) complex could be co-purified (performed by Michaela Hellwig), revealing that the small stretch of amino acids ranging from 204-214 is required for binding of Atg16L1 to Rab33B. The published Rab33B-binding domain comprises residues 141-265 of Atg16L1. Our results demonstrate the significance of residues 141214 for Rab33B binding, whereas the C-terminal part of the published binding site is not required for the interaction.

It was also observed by Itoh et al. 2008 that overexpression of the Rab33B-binding domain of Atg16L1 inhibited autophagosome formation and also resulted in partial Atg16L1 localization to the Golgi where Rab33B is normally localized (Zheng 1998). It was hypothesized by Itoh et al. (2008) that Rab33B could be delivering lipids to the growing autophagosome and play a role in the regulation of autophagy. The Atg16L1 coiled coil domain could be a tethering factor to target Rab33B vesicles to the expanding isolation membranes. However, this mechanism would be specialized for higher eukaryotes as no Rab33B homologue has been identified in yeast.

\subsection{Characterization of phosphoinositide binding of Atg5 Atg16 complexes}

\subsubsection{Atg5 Atg16(L1) binds phosphoinositides}

In this study, I showed for the first time that the mammalian Atg5 Atg16 complex binds directly to membranes through interacting with phosphatidylinositol-phosphates (PIPs). The mammalian and yeast Atg5 Atg16 complexes preferentially bind to PI(3)P and $\mathrm{PI}(5) \mathrm{P}$. One PIP, PI(3)P is enriched in autophagosomal membranes through the action of the PI(3) kinase complex I and is essential for localization of the Atg12Atg5 Atg16 complex to growing autophagosomes (Schu 1993, Suzuki 2001, Mizushima 2001, Suzuki 2007a, Obara 2008). We speculate that the Atg12-Atg5 Atg16L1 complex 
could be recruited to growing isolation membranes through a direct interaction with $\mathrm{PI}(3) \mathrm{P}$ in the membrane.

PI(3)P binding of the mammalian Atg5 Atg16L1(1-265) complex was shown by protein lipid overlay assays and liposome flotation experiments. Future work will include using co-flotation assays with liposomes to confirm the interaction of the mammalian Atg5 Atg16L1(1-265) complex with PI(3,5) $\mathrm{P}_{2}$, which was observed with PIP strip protein lipid overlay assays. Although an interaction between the Atg5 Atg16L1(1-265) complex and $\mathrm{PI}(4,5) \mathrm{P}_{2}$ was not observed with PIP strip protein lipid overlay assays, the Atg5 Atg16L1(1-265) complex bound liposomes containing $\mathrm{PI}(4,5) \mathrm{P}_{2}$ during co-flotation experiments. The phospholipid $\mathrm{PI}(4,5) \mathrm{P}_{2}$ is located primarily on the plasma membrane (Martin 2001). Two recent studies have observed Atg16L1 association with the plasma membrane via interaction with Nod2 and clathrin (Ravikumar 2010, Travassos 2010, Cooney 2010). Affinity for $\mathrm{PI}(4,5) \mathrm{P}_{2}$ may aid in this recruitment and localization.

The Atg5 Atg16 complexes bind to various PIPs. In the case of the mammalian Atg5 Atg16L1 complex, a preference for $\mathrm{PI}(3) \mathrm{P}$ was observed whereas the yeast Atg5 Atg16 complex demonstrated the highest affinity for PI(5)P. To further characterize the specificity of phosphoinositide binding, co-localization studies using the markers for $\mathrm{PI}(3) \mathrm{P}$ and $\mathrm{PI}(5) \mathrm{P}$ could be performed in vivo. GFP-2xFYVE could be used as a marker for PI(3)P (Gillooly 2000) and the PHD domain of ING2 could act as the marker for PI(5)P (Gozani 2003).

\subsubsection{Atg5 Atg16(L1) binds PIPs through a non-classical binding domain}

As neither Atg5 or Atg16L1 contain any canonical PI(3)P binding motifs like a FYVE or a PX domain, we set out to determine the region responsible for lipid binding. As there are many basic residues in the N-terminus of Atg16, we speculated that binding to negatively charged PI(3)P could be mediated by a basic patch. We identified several basic residues in the N-termini of both murine Atg16L1 and yeast Atg16 despite the fact that these homologues are not highly conserved (see Figure 3.46). When the murine Atg16L1 residues K14, R15, and R22 or the yeast Atg16 residues R9, K10, and K11 are mutated to nonpolar alanines, binding of the Atg5 Atg16(L1) complex to PIPs is impaired. Secondary structure prediction methods indicate that these residues are part of an N-terminal $\alpha$-helix. However, in the structure of Atg5 Atg16(1-57) solved by Matsushita et al. (2007), the N-terminal residues forming this predicted $\alpha$-helix were disordered. 
We observed that the N-terminal region of Atg16L1 comprising the predicted $\alpha$ helix is insufficient for lipid binding but that its deletion also inhibits lipid binding. Additionally, dimerization of the Atg5 Atg16 complex strengthens the interaction of both the yeast and mammalian Atg5 Atg16 complex with PIPs. Complex formation between Atg5 and Atg16L1 is required for PIP binding. This is in agreement with previous data showing that Atg5 is required for Atg16 localization and that Atg5 targeting to the PAS is dependent upon Atg16 (Suzuki 2001, Mizushima 2003, Suzuki 2007a). Matsushita et al. (2007) also observed that the mutation of residues required for association of Atg5 and Atg16 prompted mislocalization.

This is the first example for a PIP-binding complex. In all other known PIPbinding proteins, association with the lipid is mediated by a single protein even if multiple domains in that protein are required to increase affinity.

The involvement of the predicted basic N-terminal helix of Atg16 in PIP binding might resemble the role of the N-terminal helix of the epsin ENTH domain for the interaction with $\mathrm{PI}(4,5) \mathrm{P}_{2}$. In the epsin ENTH structure determined without the PIP, the first helix is flexible and disordered (Ford 2002). However in the structure, where the protein was co-crystallized with $\operatorname{IP}(1,4,5)_{3}$, the soluble headgroup of $\mathrm{PI}(4,5) \mathrm{P}_{2}$, this helix swings in and contributes to the positively charged cleft into which the inositol headgroup binds (see Fig. 4.1) (Ford 2002).

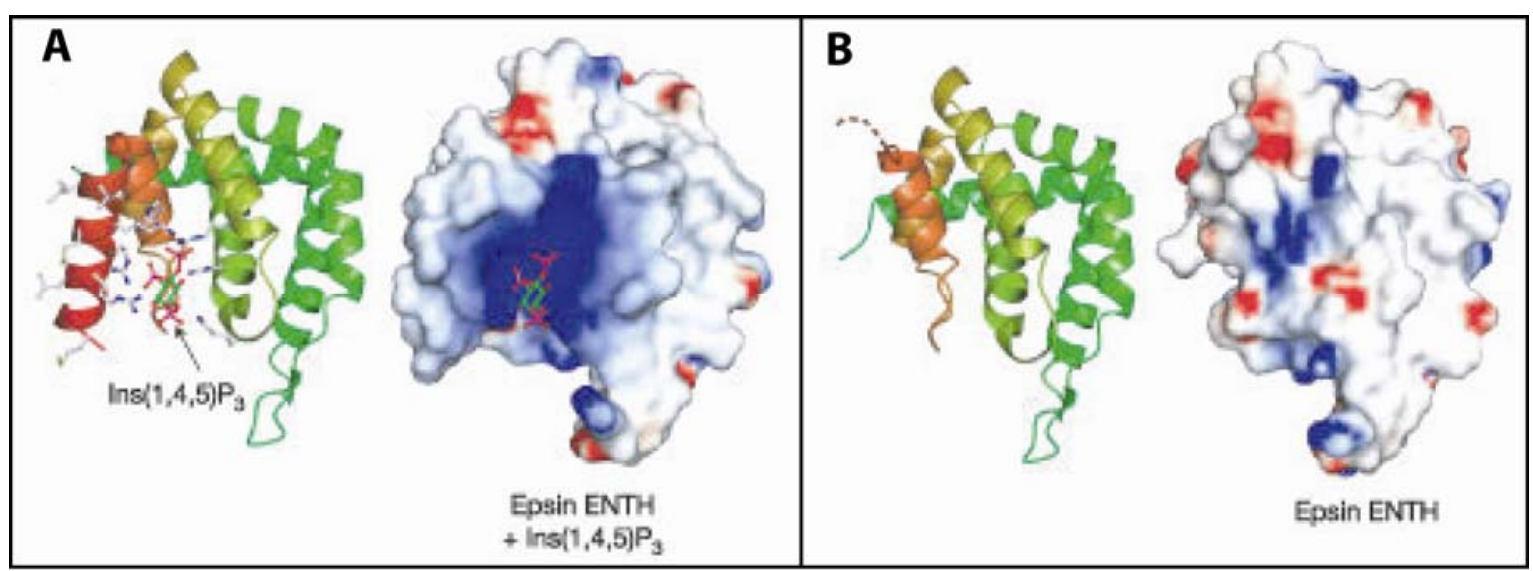

Figure 4.1: Structures of epsin in the presence and absence of the headgroup of $\mathrm{PI}(4,5) \mathrm{P}_{\mathbf{2}}$. Ribbon diagrams and surface electrostatic potentials (red - $10 \mathrm{kT} \mathrm{e}^{-1}$; blue $+10 \mathrm{kT} \mathrm{e}^{-1}$ ) of the ENTH domain of epsin (A) bound to IP(1,4,5) 3 (PDB ID: $1 \mathrm{H0A}$, Ford 2002), and (B) in the absence of the inositol headgroup of $\mathrm{PI}(4,5) \mathrm{P}_{2}$ ((PDB ID: 1EDU, Hyman 2000) where no basic cleft is formed. Adapted from Ford 2002.

To elucidate phosphoinositide binding by the Atg5 Atg16 complex, I tried to cocrystallize the soluble headgroup of PI(3)P with the mammalian Atg5 Atg16L1 complex. The best crystals of the co-crystallized mammalian Atg5 Atg16L1(1-70) complex 
diffracted only to $6 \AA$ resolution. Since the yeast Atg5 Atg 16 complexes published by Matsushita et al. $(2006,2007)$ diffracted up to $2.0 \AA$ A resolution, I tried to reproduce the published crystallization conditions of yeast Atg5 Atg16(1-46) and Atg5 Atg16(1-57) and soak the inositol diphosphate headgroup into the crystals or co-crystallize the complex in the presence of the inositol diphosphate headgroup, respectively. Diffraction data up to 2.3 and $1.8 \AA$ resolution were collected for the Atg5 Atg16(1-46) and Atg5 Atg16(1-57) complexes respectively. However, no electron density was observed that could be attributed to the ligand.

A possible explanation for lack of binding of the inositol headgroup to the Atg5 Atg16 complex could be that the interaction of Atg5 Atg16 with PI(3)P may extend past the soluble inositol headgroup and require additional hydrophobic contacts with the interfacial region where the lipid phosphate group is located. To test this hypothesis, PIP strip protein lipid overlay assays will be carried out where either the $\operatorname{IP}(1,5)_{2}$ headgroup, the soluble short lipid chain PI(5)P (diC8), or the long lipid chain PI(5)P (diC16) incorporated into liposomes will be mixed with the yeast Atg5 Atg16(1-67) complex to investigate the extent of competition in binding to the PIPs spotted on the membrane. These experiments will also be performed using the various soluble and lipidated forms of $\mathrm{PI}(3) \mathrm{P}$.

If the soluble inositol headgroup or soluble, lipidated form of PI(5)P or PI(3)P effectively competes with PI(5)P and PI(3)P spotted on the PIP strip membrane, I plan to co-crystallize and soak yeast Atg5 Atg16(1-67) with these ligands as this complex has a higher binding affinity to PIPs compared to Atg5 Atg16(1-46) and Atg5 Atg16(1-57) (Figure 3.44).

\subsubsection{Implications of PIP binding for autophagosome formation}

We speculate that by PIP binding of Atg5 Atg16, the Atg12-Atg5 Atg16 complex could be targeted to the isolation membrane since it is enriched in PI(3)P. Dimerization of the Atg5 Atg16 complex could be the important to achieving high avidity binding to $\mathrm{PI}(3) \mathrm{P}$ present in the growing autophagosomal membranes and be a possible explanation why oligomerization of the Atg12-Atg5 Atg16 complex is essential for autophagosome formation.

An example for the importance of multimerization of a protein for PIP binding is EEA1. EEA1 has weak binding affinity to PI(3)P in its monomeric form. Dimerization of EEA1 via its coiled coil domain causes it to bind two molecules of PI(3)P in the 
membrane. This increased avidity has an additive effect on the binding energy and the dissociation constant is reduced (Dumas 2001, reviewed in Lemmon 2008).

The interaction of the Atg12-Atg5 Atg16 complex with PI(3)P in autophagosomal membranes would also explain another observed behavior of the complex at the growing autophagosomal membrane. Mizushima et al. (2001) showed that the Atg12-Atg5 Atg16 complex dissociates from the autophagosomal membrane either just before or after fusion of the leading edges to form the autophagosome. Dynamics of PI(3)P in the membrane is regulated by several PI(3)P phosphatases (Vergne 2009, Taguchi-Atarashi 2010). Hydrolysis of PI(3)P in the growing isolation membranes could therefore limit expansion mediated by the Atg12-Atg5 Atg16 complex. In fact, overexpression of the PI(3)P phosphatase Myotubularin-related phosphatase 3 (MTMR3) led to the formation of smaller autophagosomes (Taguchi-Atarashi 2010).

I demonstrated that the yeast and murine Atg5 Atg16 complexes directly bind phosphoinositides and identified mutants where this interaction is abolished. To test the functional importance of PIP binding of Atg5 Atg16, in vivo studies need to be performed. I want to transfect cells with the Atg16 mutants and analyze their localization and whether autophagosome formation is affected. 


\section{SUMMARY AND OUTLOOK}

During this project, I characterized the mammalian Atg5 Atg16L1 complex which is essential for expansion of the isolation membrane during autophagosome formation. I investigated its oligomerization state and grew crystals of the dimerized mammalian complex, which diffracted to only $5 \AA$ resolution. Using two independent methods, I showed for the first time that the mammalian Atg5 Atg16L1 complex binds phosphoinositides, including $\mathrm{PI}(3) \mathrm{P}$ which is enriched in isolation membranes. Furthermore, I also demonstrated phosphoinositide binding of the yeast Atg5 Atg16 complex, making it an evolutionarily conserved property of the Atg5 Atg16 complex. Dimerization of the Atg5 Atg16 complex enhances binding affinity to PIPs.

In contrast to other PIP-binding proteins where the domains required for binding are comprised in a single protein, complex formation between Atg5 and Atg16 is required to mediate phosphoinositide binding. I demonstrated that basic residues at the $\mathrm{N}$-terminus of Atg16 play an essential role in PIP binding. Mutations of murine Atg16L1 basic residues K14, R15, and R22 to alanines impair PIP binding. Mutations of yeast Atg16L1 basic residues $\mathrm{K} 10$ and $\mathrm{K} 11$ to alanines also abolished interaction of the yeast Atg5 Atg16(1-67) complex with PIPs.

To gain structural insights into PIP binding, the soluble inositol diphosphate headgroup was either co-crystallized with the mammalian and yeast Atg5 Atg16 complexes or soaked into crystals of the yeast Atg5 Atg16 complex. High resolution datasets up to $1.8 \AA$ resolution were collected, however no ligand binding was observed. I want to perform these experiments using PIP analogues with short fatty acid chains in case that more than the inositol headgroup is required for efficient binding. Furthermore, I will attempt to co-crystallize the yeast Atg5 Atg16(1-67) complex with the PI(5)P analogue since this complex has a higher affinity for PIPs than the $\operatorname{Atg} 5 \sim \operatorname{Atg} 16(1-46)$ and Atg5 Atg16(1-57) complexes, which do not contain any part of the coiled coil domain of Atg16.

In our future work we plan to use cell models to elucidate the role of N-terminal basic residues of Atg16 in the localization of the Atg5 Atg16 complex to autophagosomal membranes. I already cloned N- and C-terminal EmGFP-Atg16L1 constructs that include the wild-type and K14A mutation. These will be co-transfected with an N-terminal FLAG-Atg5 construct into mammalian cells. Autophagy will be stimulated using the drug 
rapamycin, and the localization of the Atg5 Atg16L1 wild-type and mutant complexes will be compared.

We also want to collaborate with Prof. Dr. Michael Thumm to obtain in vivo data on the effects of the mutation of basic N-terminal residues of yeast Atg16. We want to investigate the effects of Atg16 mutants deficient in PIP binding on autophagy in yeast. GFP-Atg16 constructs, both wild-type and N-terminal mutants, will be transformed into $\operatorname{atg} 164$ yeast strains. The localization of GFP-tagged Atg16 will be analyzed and autophagic activity can be monitored, for example by measuring the lipidation of Atg8. These experiments would help to elucidate the functional importance of PIP binding by the Atg5 Atg16 complex.

I also tried to crystallize Atg16L1 in complex with one of its interaction partners, the GTPase Rab33B. Crystals of the Rab33B(30-202)Q92L Atg16L1(141-265) complex were obtained but did not diffract. The project was followed up by our technician Michaela Hellwig. She prepared and crystallized Rab33B(30-202)Q92L with the following Atg16 constructs: (141-214), (141-224), (125-214), (125-224), or (125-234). However, crystals could not be grown for any of these complexes. A next step could be to perform large scale limited proteolysis on the Rab33B(30-202)Q92L Atg16L1(141-265) complex, which was able to be crystallized, and to purify the formed cleavage product by either anion exchange chromatography or gel filtration purification. Flexible portions would be cleaved off and the stable fragments forming the complex might be more ordered and yield better diffracting crystals. 


\section{BIBLIOGRAPHY}

Axe, E. L., S. A. Walker, et al. (2008). "Autophagosome formation from membrane compartments enriched in phosphatidylinositol 3-phosphate and dynamically connected to the endoplasmic reticulum." J Cell Biol 182(4): 685-701.

Barth, H., K. Meiling-Wesse, et al. (2001). "Autophagy and the cytoplasm to vacuole targeting pathway both require Aut 10p." FEBS Lett 508(1): 23-28.

Barth, H., K. Meiling-Wesse, et al. (2002). "Mailp is essential for maturation of proaminopeptidase I but not for autophagy." FEBS Lett 512(1-3): 173-179.

Beau, I., M. Mehrpour, et al. (2011). "Autophagosomes and human diseases." Int J Biochem Cell Biol.

Behrends, C., M. E. Sowa, et al. (2010). "Network organization of the human autophagy system." Nature 466(7302): 68-76.

Bjorkoy, G., T. Lamark, et al. (2005). "p62/SQSTM1 forms protein aggregates degraded by autophagy and has a protective effect on huntingtin-induced cell death." J Cell Biol 171(4): 603-614.

Blommaart, E. F., U. Krause, et al. (1997). "The phosphatidylinositol 3-kinase inhibitors wortmannin and LY294002 inhibit autophagy in isolated rat hepatocytes." Eur J Biochem 243(1-2): 240-246.

Bravo, J., D. Karathanassis, et al. (2001). "The crystal structure of the PX domain from p40(phox) bound to phosphatidylinositol 3-phosphate." Mol Cell 8(4): 829-839.

Burguete, A. S., T. D. Fenn, et al. (2008). "Rab and Arl GTPase family members cooperate in the localization of the golgin GCC185." Cell 132(2): 286-298.

Burman, C. and N. T. Ktistakis (2010). "Regulation of autophagy by phosphatidylinositol 3-phosphate." FEBS Lett 584(7): 1302-1312.

Bursch, W. (2001). "The autophagosomal-lysosomal compartment in programmed cell death." Cell Death Differ 8(6): 569-581.

Cadwell, K., K. K. Patel, et al. (2010). "Virus-plus-susceptibility gene interaction determines Crohn's disease gene Atg16L1 phenotypes in intestine." Cell 141(7): 11351145 .

Cao, Y., H. Cheong, et al. (2008). "In vivo reconstitution of autophagy in Saccharomyces cerevisiae." J Cell Biol 182(4): 703-713.

Chayen, N. E. (1997). "The role of oil in macromolecular crystallization." Structure 5(10): 1269-1274.

Cheng, Y., F. Qiu, et al. (2008). "Apoptosis-suppressing and autophagy-promoting effects of calpain on oridonin-induced L929 cell death." Arch Biochem Biophys 475(2): 148155.

Cheong, H., U. Nair, et al. (2008). "The Atg1 kinase complex is involved in the regulation of protein recruitment to initiate sequestering vesicle formation for nonspecific autophagy in Saccharomyces cerevisiae." Mol Biol Cell 19(2): 668-681.

Cho, J. H. (2008). "The genetics and immunopathogenesis of inflammatory bowel disease." Nat Rev Immunol 8(6): 458-466.

Ciechanover, A., A. Orian, et al. (2000). "Ubiquitin-mediated proteolysis: biological regulation via destruction." Bioessays 22(5): 442-451. 
Cole, C., J. D. Barber, et al. (2008). "The Jpred 3 secondary structure prediction server." Nucleic Acids Res 36(Web Server issue): W197-201.

Collaborative Computational Project, N. (1994). "The CCP4 suite: programs for protein crystallography." Acta Crystallogr D Biol Crystallogr 50(Pt 5): 760-763.

Cong, J., D. E. Goll, et al. (1989). "The role of autolysis in activity of the Ca2+-dependent proteinases (mu-calpain and m-calpain)." J Biol Chem 264(17): 10096-10103.

Consortium, W. T. C. C. (2007). "Genome-wide association study of 14,000 cases of seven common diseases and 3,000 shared controls." Nature 447(7145): 661-678.

Cooney, R., J. Baker, et al. (2010). "NOD2 stimulation induces autophagy in dendritic cells influencing bacterial handling and antigen presentation." Nat Med 16(1): 90-97.

Dagert, M. and S. D. Ehrlich (1979). "Prolonged incubation in calcium chloride improves the competence of Escherichia coli cells." Gene 6(1): 23-28.

Dale, G. E., C. Oefner, et al. (2003). "The protein as a variable in protein crystallization." J Struct Biol 142(1): 88-97.

de Vet, E. C. and H. van den Bosch (2000). "Alkyl-dihydroxyacetonephosphate synthase." Cell Biochem Biophys 32 Spring: 117-121.

Delano, W. L. (Delano Scientific). http://www.pymol.org.

Delgado, M. A., R. A. Elmaoued, et al. (2008). "Toll-like receptors control autophagy." EMBO J 27(7): 1110-1121.

Demarchi, F., C. Bertoli, et al. (2006). "Calpain is required for macroautophagy in mammalian cells." J Cell Biol 175(4): 595-605.

Dice, J. F. (1990). "Peptide sequences that target cytosolic proteins for lysosomal proteolysis." Trends Biochem Sci 15(8): 305-309.

Dice, J. F. (2007). "Chaperone-mediated autophagy." Autophagy 3(4): 295-299.

Diederichs, K. and P. A. Karplus (1997). "Improved R-factors for diffraction data analysis in macromolecular crystallography." Nat Struct Biol 4(4): 269-275.

Dove, S. K., R. C. Piper, et al. (2004). "Svp1p defines a family of phosphatidylinositol 3,5-bisphosphate effectors." EMBO J 23(9): 1922-1933.

Dower, W. J., J. F. Miller, et al. (1988). "High efficiency transformation of E. coli by high voltage electroporation." Nucleic Acids Res 16(13): 6127-6145.

Dowler, S., G. Kular, et al. (2002). "Protein lipid overlay assay." Sci STKE 2002(129): pl6.

Dubendorff, J. W. and F. W. Studier (1991). "Controlling basal expression in an inducible $\mathrm{T} 7$ expression system by blocking the target $\mathrm{T} 7$ promoter with lac repressor." J Mol Biol 219(1): 45-59.

Dumas, J. J., E. Merithew, et al. (2001). "Multivalent endosome targeting by homodimeric EEA1." Mol Cell 8(5): 947-958.

Eathiraj, S., A. Mishra, et al. (2006). "Structural basis for Rab11-mediated recruitment of FIP3 to recycling endosomes." J Mol Biol 364(2): 121-135.

Eathiraj, S., X. Pan, et al. (2005). "Structural basis of family-wide Rab GTPase recognition by rabenosyn-5." Nature 436(7049): 415-419.

Emsley, P. and K. Cowtan (2004). "Coot: model-building tools for molecular graphics." Acta Crystallogr D Biol Crystallogr 60(Pt 12 Pt 1): 2126-2132. 
Emsley, P., B. Lohkamp, et al. (2010). "Features and development of Coot." Acta Crystallogr D Biol Crystallogr 66(Pt 4): 486-501.

Farre, J. C., R. Krick, et al. (2009). "Turnover of organelles by autophagy in yeast." Curr Opin Cell Biol 21(4): 522-530.

Ferguson, C. J., G. M. Lenk, et al. (2009). "Defective autophagy in neurons and astrocytes from mice deficient in PI(3,5)P2." Hum Mol Genet 18(24): 4868-4878.

Fleming, A., T. Noda, et al. (2011). "Chemical modulators of autophagy as biological probes and potential therapeutics." Nat Chem Biol 7(1): 9-17.

Ford, M. G., I. G. Mills, et al. (2002). "Curvature of clathrin-coated pits driven by epsin." Nature 419(6905): 361-366.

Fujioka, Y., N. N. Noda, et al. (2008). "Crystallization of the coiled-coil domain of Atg16 essential for autophagy." Acta Crystallogr Sect F Struct Biol Cryst Commun 64(Pt 11): 1046-1048.

Fujioka, Y., N. N. Noda, et al. (2010). "Dimeric coiled-coil structure of Saccharomyces cerevisiae Atg16 and its functional significance in autophagy." J Biol Chem 285(2): 1508-1515.

Fujita, N., M. Hayashi-Nishino, et al. (2008b). "An Atg4B mutant hampers the lipidation of LC3 paralogues and causes defects in autophagosome closure." Mol Biol Cell 19(11): 4651-4659.

Fujita, N., T. Itoh, et al. (2008a). "The Atg16L complex specifies the site of LC3 lipidation for membrane biogenesis in autophagy." Mol Biol Cell 19(5): 2092-2100.

Fujita, N., T. Saitoh, et al. (2009). "Differential involvement of Atg16L1 in Crohn disease and canonical autophagy: analysis of the organization of the Atg16L1 complex in fibroblasts." J Biol Chem 284(47): 32602-32609.

Funakoshi, T., A. Matsuura, et al. (1997). "Analyses of APG13 gene involved in autophagy in yeast, Saccharomyces cerevisiae." Gene 192(2): 207-213.

Geng, J., M. Baba, et al. (2008b). "Quantitative analysis of autophagy-related protein stoichiometry by fluorescence microscopy." J Cell Biol 182(1): 129-140.

Geng, J. and D. J. Klionsky (2008a). "The Atg8 and Atg12 ubiquitin-like conjugation systems in macroautophagy. 'Protein modifications: beyond the usual suspects' review series." EMBO Rep 9(9): 859-864.

Geng, J., U. Nair, et al. (2010). "Post-Golgi Sec proteins are required for autophagy in Saccharomyces cerevisiae." Mol Biol Cell 21(13): 2257-2269.

Gillooly, D. J., I. C. Morrow, et al. (2000). "Localization of phosphatidylinositol 3phosphate in yeast and mammalian cells." EMBO J 19(17): 4577-4588.

Goll, D. E., V. F. Thompson, et al. (2003). "The calpain system." Physiol Rev 83(3): 731801 .

Gordon, P. B., I. Holen, et al. (1993). "Dependence of hepatocytic autophagy on intracellularly sequestered calcium." J Biol Chem 268(35): 26107-26112.

Gozani, O., P. Karuman, et al. (2003). "The PHD finger of the chromatin-associated protein ING2 functions as a nuclear phosphoinositide receptor." Cell 114(1): 99-111.

Guan, J., P. E. Stromhaug, et al. (2001). "Cvt18/Gsa12 is required for cytoplasm-tovacuole transport, pexophagy, and autophagy in Saccharomyces cerevisiae and Pichia pastoris." Mol Biol Cell 12(12): 3821-3838. 
Hailey, D. W., A. S. Rambold, et al. (2010). "Mitochondria supply membranes for autophagosome biogenesis during starvation." Cell 141(4): 656-667.

Hampe, J., A. Franke, et al. (2007). "A genome-wide association scan of nonsynonymous SNPs identifies a susceptibility variant for Crohn disease in ATG16L1." Nat Genet 39(2): 207-211.

Hanada, T., N. N. Noda, et al. (2007). "The Atg12-Atg5 conjugate has a novel E3-like activity for protein lipidation in autophagy." J Biol Chem 282(52): 37298-37302.

Hanada, T. and Y. Ohsumi (2005). "Structure-function relationship of Atg12, a ubiquitinlike modifier essential for autophagy." Autophagy 1(2): 110-118.

Harding, T. M., K. A. Morano, et al. (1995). "Isolation and characterization of yeast mutants in the cytoplasm to vacuole protein targeting pathway." J Cell Biol 131(3): 591-602.

Hayakawa, A., S. J. Hayes, et al. (2004). "Structural basis for endosomal targeting by FYVE domains." J Biol Chem 279(7): 5958-5966.

Hayashi-Nishino, M., N. Fujita, et al. (2009). "A subdomain of the endoplasmic reticulum forms a cradle for autophagosome formation." Nat Cell Biol 11(12): 1433-1437.

Hemelaar, J., V. S. Lelyveld, et al. (2003). "A single protease, Apg4B, is specific for the autophagy-related ubiquitin-like proteins GATE-16, MAP1-LC3, GABARAP, and Apg8L." J Biol Chem 278(51): 51841-51850.

Higuchi, R., B. Krummel, et al. (1988). "A general method of in vitro preparation and specific mutagenesis of DNA fragments: study of protein and DNA interactions." Nucleic Acids Res 16(15): 7351-7367.

Hochuli, E. (1990). "Purification of recombinant proteins with metal chelate adsorbent." Genet Eng (N Y) 12: 87-98.

Huang, W. P., S. V. Scott, et al. (2000). "The itinerary of a vesicle component, Aut $7 \mathrm{p} / \mathrm{Cvt} 5 \mathrm{p}$, terminates in the yeast vacuole via the autophagy/Cvt pathways." J Biol Chem 275(8): 5845-5851.

Hugot, J. P., M. Chamaillard, et al. (2001). "Association of NOD2 leucine-rich repeat variants with susceptibility to Crohn's disease." Nature 411(6837): 599-603.

Hyman, J., H. Chen, et al. (2000). "Epsin 1 undergoes nucleocytosolic shuttling and its eps15 interactor $\mathrm{NH}(2)$-terminal homology (ENTH) domain, structurally similar to Armadillo and HEAT repeats, interacts with the transcription factor promyelocytic leukemia Zn(2)+ finger protein (PLZF)." J Cell Biol 149(3): 537-546.

Ichimura, Y., T. Kirisako, et al. (2000). "A ubiquitin-like system mediates protein lipidation." Nature 408(6811): 488-492.

Itoh, T., N. Fujita, et al. (2008). "Golgi-resident small GTPase Rab33B interacts with Atg16L and modulates autophagosome formation." Mol Biol Cell 19(7): 2916-2925.

Jagoe, W. N., A. J. Lindsay, et al. (2006). "Crystal structure of rab11 in complex with rab11 family interacting protein 2." Structure 14(8): 1273-1283.

Jenny, R. J., K. G. Mann, et al. (2003). "A critical review of the methods for cleavage of fusion proteins with thrombin and factor Xa." Protein Expr Purif 31(1): 1-11.

Jounai, N., F. Takeshita, et al. (2007). "The Atg5 Atg12 conjugate associates with innate antiviral immune responses." Proc Natl Acad Sci U S A 104(35): 14050-14055.

Ju, J. S., R. A. Fuentealba, et al. (2009). "Valosin-containing protein (VCP) is required for autophagy and is disrupted in VCP disease." J Cell Biol 187(6): 875-888. 
Kabeya, Y., Y. Kamada, et al. (2005). "Atg17 functions in cooperation with Atg1 and Atg13 in yeast autophagy." Mol Biol Cell 16(5): 2544-2553.

Kabeya, Y., T. Kawamata, et al. (2007). "Cis1/Atg31 is required for autophagosome formation in Saccharomyces cerevisiae." Biochem Biophys Res Commun 356(2): $405-$ 410.

Kabeya, Y., N. Mizushima, et al. (2004). "LC3, GABARAP and GATE16 localize to autophagosomal membrane depending on form-II formation." J Cell Sci 117(Pt 13): 2805-2812.

Kabeya, Y., N. N. Noda, et al. (2009). "Characterization of the Atg17-Atg29-Atg31 complex specifically required for starvation-induced autophagy in Saccharomyces cerevisiae." Biochem Biophys Res Commun 389(4): 612-615.

Kabsch, W. (1988). "Automatic-Indexing of Rotation Diffraction Patterns." Journal of Applied Crystallography 21: 67-71.

Kabsch, W. (1993). "Automatic Processing of Rotation Diffraction Data from Crystals of Initially Unknown Symmetry and Cell Constants." Journal of Applied Crystallography 26: $795-800$.

Kabsch, W. (2010a). "Xds." Acta Crystallogr D Biol Crystallogr 66(Pt 2): 125-132.

Kabsch, W. (2010b). "Integration, scaling, space-group assignment and post-refinement." Acta Crystallogr D Biol Crystallogr 66(Pt 2): 133-144.

Kamada, Y., T. Funakoshi, et al. (2000). "Tor-mediated induction of autophagy via an Apg1 protein kinase complex." J Cell Biol 150(6): 1507-1513.

Kantardjieff, K. A. and B. Rupp (2003). "Matthews coefficient probabilities: Improved estimates for unit cell contents of proteins, DNA, and protein-nucleic acid complex crystals." Protein Sci 12(9): 1865-1871.

Kawamata, T., Y. Kamada, et al. (2008). "Organization of the pre-autophagosomal structure responsible for autophagosome formation." Mol Biol Cell 19(5): 2039-2050.

Kerscher, O., R. Felberbaum, et al. (2006). "Modification of proteins by ubiquitin and ubiquitin-like proteins." Annu Rev Cell Dev Biol 22: 159-180.

Kihara, A., T. Noda, et al. (2001). "Two distinct Vps34 phosphatidylinositol 3-kinase complexes function in autophagy and carboxypeptidase $\mathrm{Y}$ sorting in Saccharomyces cerevisiae." J Cell Biol 152(3): 519-530.

Kim, J., V. M. Dalton, et al. (1999). "Apg7p/Cvt2p is required for the cytoplasm-tovacuole targeting, macroautophagy, and peroxisome degradation pathways." Mol Biol Cell 10(5): 1337-1351.

Kirisako, T., Y. Ichimura, et al. (2000). "The reversible modification regulates the membrane-binding state of Apg8/Aut7 essential for autophagy and the cytoplasm to vacuole targeting pathway." J Cell Biol 151(2): 263-276.

Klionsky, D. J. (2007). "Autophagy: from phenomenology to molecular understanding in less than a decade." Nat Rev Mol Cell Biol 8(11): 931-937.

Klionsky, D. J., J. M. Cregg, et al. (2003). "A unified nomenclature for yeast autophagyrelated genes." Dev Cell 5(4): 539-545.

Komatsu, M. and Y. Ichimura (2010). "Selective autophagy regulates various cellular functions." Genes Cells 15(9): 923-933. 
Komatsu, M., I. Tanida, et al. (2001). "The C-terminal region of an Apg7p/Cvt2p is required for homodimerization and is essential for its E1 activity and E1-E2 complex formation." J Biol Chem 276(13): 9846-9854.

Komatsu, M., S. Waguri, et al. (2006). "Loss of autophagy in the central nervous system causes neurodegeneration in mice." Nature 441(7095): 880-884.

Komatsu, M., S. Waguri, et al. (2007). "Homeostatic levels of p62 control cytoplasmic inclusion body formation in autophagy-deficient mice." Cell 131(6): 1149-1163.

Komatsu, M., S. Waguri, et al. (2005). "Impairment of starvation-induced and constitutive autophagy in Atg7-deficient mice." J Cell Biol 169(3): 425-434.

Kovacs, A. L., G. Rez, et al. (2000). "Autophagy in the epithelial cells of murine seminal vesicle in vitro. Formation of large sheets of nascent isolation membranes, sequestration of the nucleus and inhibition by wortmannin and 3-ethyladenine." Cell Tissue Res 302(2): 253-261.

Krick, R., S. Bremer, et al. (2010). "Cdc48/p97 and Shp1/p47 regulate autophagosome biogenesis in concert with ubiquitin-like Atg8." J Cell Biol 190(6): 965-973.

Krick, R., S. Henke, et al. (2008b). "Dissecting the localization and function of Atg18, Atg21 and Ygr223c." Autophagy 4(7): 896-910.

Krick, R., Y. Muehe, et al. (2008a). "Piecemeal Microautophagy of the Nucleus Requires the Core Macroautophagy Genes." Molecular Biology of the Cell 19(10): 4492-4505.

Krick, R., J. Tolstrup, et al. (2006). "The relevance of the phosphatidylinositolphosphatbinding motif FRRGT of Atg18 and Atg21 for the Cvt pathway and autophagy." Febs Letters 580(19): 4632-4638.

Kroemer, G., G. Marino, et al. (2010). "Autophagy and the integrated stress response." Mol Cell 40(2): 280-293.

Kuballa, P., A. Huett, et al. (2008). "Impaired autophagy of an intracellular pathogen induced by a Crohn's disease associated ATG16L1 variant." PLoS One 3(10): e3391.

Kuma, A., N. Mizushima, et al. (2002). "Formation of the approximately 350-kDa Apg12Apg5.Apg 16 multimeric complex, mediated by Apg16 oligomerization, is essential for autophagy in yeast." J Biol Chem 277(21): 18619-18625.

Kumanomidou, T., T. Mizushima, et al. (2006). "The crystal structure of human Atg4b, a processing and de-conjugating enzyme for autophagosome-forming modifiers." $\mathrm{J}$ Mol Biol 355(4): 612-618.

Laemmli, U. K. (1970). "Cleavage of structural proteins during the assembly of the head of bacteriophage T4." Nature 227(5259): 680-685.

Legakis, J. E., W. L. Yen, et al. (2007). "A cycling protein complex required for selective autophagy." Autophagy 3(5): 422-432.

Lemmon, M. A. (2008). "Membrane recognition by phospholipid-binding domains." Nat Rev Mol Cell Biol 9(2): 99-111.

Levine, B. and V. Deretic (2007). "Unveiling the roles of autophagy in innate and adaptive immunity." Nat Rev Immunol 7(10): 767-777.

Lietzke, S. E., S. Bose, et al. (2000). "Structural basis of 3-phosphoinositide recognition by pleckstrin homology domains." Mol Cell 6(2): 385-394.

Linding, R., L. J. Jensen, et al. (2003). "Protein disorder prediction: implications for structural proteomics." Structure 11(11): 1453-1459. 
Lo, M. C., A. Aulabaugh, et al. (2004). "Evaluation of fluorescence-based thermal shift assays for hit identification in drug discovery." Anal Biochem 332(1): 153-159.

Lupas, A. (1996). "Coiled coils: new structures and new functions." Trends Biochem Sci 21(10): 375-382.

Lupas, A., M. Van Dyke, et al. (1991). "Predicting coiled coils from protein sequences." Science 252(5010): 1162-1164.

Lynch-Day, M. A., D. Bhandari, et al. (2010). "Trs85 directs a Ypt1 GEF, TRAPPIII, to the phagophore to promote autophagy." Proc Natl Acad Sci U S A 107(17): 78117816.

Mandel, M. and A. Higa (1970). "Calcium-dependent bacteriophage DNA infection." J Mol Biol 53(1): 159-162.

Mann, S. S. and J. A. Hammarback (1994). "Molecular characterization of light chain 3. A microtubule binding subunit of MAP1A and MAP1B." J Biol Chem 269(15): 1149211497.

Martin, T. F. (2001). "PI(4,5)P(2) regulation of surface membrane traffic." Curr Opin Cell Biol 13(4): 493-499.

Matsunaga, K., E. Morita, et al. (2010). "Autophagy requires endoplasmic reticulum targeting of the PI3-kinase complex via Atg14L." J Cell Biol 190(4): 511-521.

Matsunaga, K., T. Saitoh, et al. (2009). "Two Beclin 1-binding proteins, Atg14L and Rubicon, reciprocally regulate autophagy at different stages." Nat Cell Biol 11(4): 385-396.

Matsuoka, K., L. Orci, et al. (1998). "COPII-coated vesicle formation reconstituted with purified coat proteins and chemically defined liposomes." Cell 93(2): 263-275.

Matsushita, M., N. N. Suzuki, et al. (2006). "Expression, purification and crystallization of the Atg5-Atg16 complex essential for autophagy." Acta Crystallogr Sect F Struct Biol Cryst Commun 62(Pt 10): 1021-1023.

Matsushita, M., N. N. Suzuki, et al. (2007). "Structure of Atg5.Atg16, a complex essential for autophagy." J Biol Chem 282(9): 6763-6772.

Matthews, B. W. (1968). "Solvent content of protein crystals." J Mol Biol 33(2): 491-497.

Matthews, B. W. (1976). "X-Ray Crystallographic Studies of Proteins." Annual Review of Physical Chemistry 27: 493-523.

Meiling-Wesse, K., H. Barth, et al. (2004). "Atg21 is required for effective recruitment of Atg8 to the preautophagosomal structure during the Cvt pathway." J Biol Chem 279(36): 37741-37750.

Mizushima, N. (2007). "Autophagy: process and function." Genes Dev 21(22): 2861-2873.

Mizushima, N., A. Kuma, et al. (2003). "Mouse Apg16L, a novel WD-repeat protein, targets to the autophagic isolation membrane with the Apg12-Apg5 conjugate." J Cell Sci 116(Pt 9): 1679-1688.

Mizushima, N., B. Levine, et al. (2008). "Autophagy fights disease through cellular selfdigestion." Nature 451(7182): 1069-1075.

Mizushima, N., T. Noda, et al. (1999). "Apg16p is required for the function of the Apg12p-Apg5p conjugate in the yeast autophagy pathway." EMBO J 18(14): 38883896. 
Mizushima, N., T. Noda, et al. (1998a). "A protein conjugation system essential for autophagy." Nature 395(6700): 395-398.

Mizushima, N., H. Sugita, et al. (1998b). "A new protein conjugation system in human. The counterpart of the yeast Apg12p conjugation system essential for autophagy." J Biol Chem 273(51): 33889-33892.

Mizushima, N., A. Yamamoto, et al. (2001). "Dissection of autophagosome formation using Apg5-deficient mouse embryonic stem cells." J Cell Biol 152(4): 657-668.

Mizushima, N., T. Yoshimori, et al. (2002). "Mouse Apg10 as an Apg12-conjugating enzyme: analysis by the conjugation-mediated yeast two-hybrid method." FEBS Lett 532(3): 450-454.

Murshudov, G. N., A. A. Vagin, et al. (1997). "Refinement of macromolecular structures by the maximum-likelihood method." Acta Crystallogr D Biol Crystallogr 53(Pt 3): 240-255.

Nakatogawa, H., Y. Ichimura, et al. (2007). "Atg8, a ubiquitin-like protein required for autophagosome formation, mediates membrane tethering and hemifusion." Cell 130(1): 165-178.

Nakatogawa, H., K. Suzuki, et al. (2009). "Dynamics and diversity in autophagy mechanisms: lessons from yeast." Nat Rev Mol Cell Biol 10(7): 458-467.

Narayan, K. and M. A. Lemmon (2006). "Determining selectivity of phosphoinositidebinding domains." Methods 39(2): 122-133.

Nettleship, J. E., J. Brown, et al. (2008). "Methods for protein characterization by mass spectrometry, thermal shift (ThermoFluor) assay, and multiangle or static light scattering." Methods Mol Biol 426: 299-318.

Nice, D. C., T. K. Sato, et al. (2002). "Cooperative binding of the cytoplasm to vacuole targeting pathway proteins, Cvt13 and Cvt20, to phosphatidylinositol 3-phosphate at the pre-autophagosomal structure is required for selective autophagy." J Biol Chem 277(33): 30198-30207.

Noda, N. N., H. Kumeta, et al. (2008). "Structural basis of target recognition by Atg8/LC3 during selective autophagy." Genes Cells 13(12): 1211-1218.

Noda, N. N., Y. Ohsumi, et al. (2009). "ATG systems from the protein structural point of view." Chem Rev 109(4): 1587-1598.

Noda, T., J. Kim, et al. (2000). "Apg9p/Cvt7p is an integral membrane protein required for transport vesicle formation in the Cvt and autophagy pathways." J Cell Biol 148(3): 465-480.

Noda, T. and Y. Ohsumi (1998). "Tor, a phosphatidylinositol kinase homologue, controls autophagy in yeast." J Biol Chem 273(7): 3963-3966.

Norman, J. M., G. M. Cohen, et al. (2010). "The in vitro cleavage of the hAtg proteins by cell death proteases." Autophagy 6(8): 1042-1056.

Notredame, C., D. G. Higgins, et al. (2000). "T-Coffee: A novel method for fast and accurate multiple sequence alignment." J Mol Biol 302(1): 205-217.

Obara, K., T. Sekito, et al. (2008). "The Atg18-Atg2 complex is recruited to autophagic membranes via phosphatidylinositol 3-phosphate and exerts an essential function." J Biol Chem 283(35): 23972-23980. 
Obara, K., T. Sekito, et al. (2006). "Assortment of phosphatidylinositol 3-kinase complexes--Atg14p directs association of complex I to the pre-autophagosomal structure in Saccharomyces cerevisiae." Mol Biol Cell 17(4): 1527-1539.

Ogawa, M., T. Yoshimori, et al. (2005). "Escape of intracellular Shigella from autophagy." Science 307(5710): 727-731.

Ohya, T., M. Miaczynska, et al. (2009). "Reconstitution of Rab- and SNARE-dependent membrane fusion by synthetic endosomes." Nature 459(7250): 1091-1097.

Pankiv, S., T. H. Clausen, et al. (2007). "p62/SQSTM1 binds directly to Atg8/LC3 to facilitate degradation of ubiquitinated protein aggregates by autophagy." J Biol Chem 282(33): 24131-24145.

Pantoliano, M. W., E. C. Petrella, et al. (2001). "High-density miniaturized thermal shift assays as a general strategy for drug discovery." J Biomol Screen 6(6): 429-440.

Paz, Y., Z. Elazar, et al. (2000). "Structure of GATE-16, membrane transport modulator and mammalian ortholog of autophagocytosis factor Aut7p." J Biol Chem 275(33): 25445-25450.

Petiot, A., E. Ogier-Denis, et al. (2000). "Distinct classes of phosphatidylinositol 3'kinases are involved in signaling pathways that control macroautophagy in HT-29 cells." J Biol Chem 275(2): 992-998.

Poirot, O., E. O'Toole, et al. (2003). "Tcoffee@igs: A web server for computing, evaluating and combining multiple sequence alignments." Nucleic Acids Res 31(13): 3503-3506.

Polson, H. E., J. de Lartigue, et al. (2010). "Mammalian Atg18 (WIPI2) localizes to omegasome-anchored phagophores and positively regulates LC3 lipidation." Autophagy 6(4).

Potterton, E., P. Briggs, et al. (2003). "A graphical user interface to the CCP4 program suite." Acta Crystallogr D Biol Crystallogr 59(Pt 7): 1131-1137.

Pyo, J. O., M. H. Jang, et al. (2005). "Essential roles of Atg5 and FADD in autophagic cell death: dissection of autophagic cell death into vacuole formation and cell death." $\mathrm{J}$ Biol Chem 280(21): 20722-20729.

Qu, X., J. Yu, et al. (2003). "Promotion of tumorigenesis by heterozygous disruption of the beclin 1 autophagy gene." J Clin Invest 112(12): 1809-1820.

Radoshevich, L. and J. Debnath (2011). "ATG12-ATG3 and mitochondria." Autophagy 7(1): 109-111.

Radoshevich, L., L. Murrow, et al. (2010). "ATG12 conjugation to ATG3 regulates mitochondrial homeostasis and cell death." Cell 142(4): 590-600.

Ravikumar, B., R. Duden, et al. (2002). "Aggregate-prone proteins with polyglutamine and polyalanine expansions are degraded by autophagy." Hum Mol Genet 11(9): 11071117.

Ravikumar, B., M. Futter, et al. (2009). "Mammalian macroautophagy at a glance." J Cell Sci 122(Pt 11): 1707-1711.

Ravikumar, B., K. Moreau, et al. (2010). "Plasma membrane contributes to the formation of pre-autophagosomal structures." Nat Cell Biol 12(8): 747-757.

Ravikumar, B., C. Vacher, et al. (2004). "Inhibition of mTOR induces autophagy and reduces toxicity of polyglutamine expansions in fly and mouse models of Huntington disease." Nat Genet 36(6): 585-595. 
Reggiori, F. and D. J. Klionsky (2006). "Atg9 sorting from mitochondria is impaired in early secretion and VFT-complex mutants in Saccharomyces cerevisiae." J Cell Sci 119(Pt 14): 2903-2911.

Reggiori, F., T. Shintani, et al. (2005). "Atg9 cycles between mitochondria and the preautophagosomal structure in yeasts." Autophagy 1(2): 101-109.

Reggiori, F., K. A. Tucker, et al. (2004). "The Atg1-Atg13 complex regulates Atg9 and Atg23 retrieval transport from the pre-autophagosomal structure." Dev Cell 6(1): 7990.

Ridley, S. H., N. Ktistakis, et al. (2001). "FENS-1 and DFCP1 are FYVE domaincontaining proteins with distinct functions in the endosomal and Golgi compartments." J Cell Sci 114(Pt 22): 3991-4000.

Rioux, J. D., R. J. Xavier, et al. (2007). "Genome-wide association study identifies new susceptibility loci for Crohn disease and implicates autophagy in disease pathogenesis." Nat Genet 39(5): 596-604.

Roberts, P., S. Moshitch-Moshkovitz, et al. (2003). "Piecemeal microautophagy of nucleus in Saccharomyces cerevisiae." Mol Biol Cell 14(1): 129-141.

Sagiv, Y., A. Legesse-Miller, et al. (2000). "GATE-16, a membrane transport modulator, interacts with NSF and the Golgi v-SNARE GOS-28." EMBO J 19(7): 1494-1504.

Saiki, R. K., D. H. Gelfand, et al. (1988). "Primer-directed enzymatic amplification of DNA with a thermostable DNA polymerase." Science 239(4839): 487-491.

Saitoh, T., N. Fujita, et al. (2008). "Loss of the autophagy protein Atg16L1 enhances endotoxin-induced IL-1beta production." Nature 456(7219): 264-268.

Schagger, H. and G. von Jagow (1987). "Tricine-sodium dodecyl sulfate-polyacrylamide gel electrophoresis for the separation of proteins in the range from 1 to $100 \mathrm{kDa}$." Anal Biochem 166(2): 368-379.

Schlumpberger, M., E. Schaeffeler, et al. (1997). "AUT1, a gene essential for autophagocytosis in the yeast Saccharomyces cerevisiae." J Bacteriol 179(4): 10681076.

Schu, P. V., K. Takegawa, et al. (1993). "Phosphatidylinositol 3-kinase encoded by yeast VPS34 gene essential for protein sorting." Science 260(5104): 88-91.

Scott, S. V., J. Guan, et al. (2001). "Cvt19 is a receptor for the cytoplasm-to-vacuole targeting pathway." Mol Cell 7(6): 1131-1141.

Seglen, P. O. and P. B. Gordon (1982). "3-Methyladenine: specific inhibitor of autophagic/lysosomal protein degradation in isolated rat hepatocytes." Proc Natl Acad Sci U S A 79(6): 1889-1892.

Sharma, S. K., D. B. Evans, et al. (1991). "Metal affinity chromatography of recombinant HIV-1 reverse transcriptase containing a human renin cleavable metal binding domain." Biotechnol Appl Biochem 14(1): 69-81.

Shintani, T., W. P. Huang, et al. (2002). "Mechanism of cargo selection in the cytoplasm to vacuole targeting pathway." Dev Cell 3(6): 825-837.

Shintani, T., N. Mizushima, et al. (1999). "Apg10p, a novel protein-conjugating enzyme essential for autophagy in yeast." EMBO J 18(19): 5234-5241.

Shintani, T., K. Suzuki, et al. (2001). "Apg2p functions in autophagosome formation on the perivacuolar structure." J Biol Chem 276(32): 30452-30460. 
Siegel, L. M. and K. J. Monty (1966). "Determination of molecular weights and frictional ratios of proteins in impure systems by use of gel filtration and density gradient centrifugation. Application to crude preparations of sulfite and hydroxylamine reductases." Biochim Biophys Acta 112(2): 346-362.

Simonsen, A., H. C. Birkeland, et al. (2004). "Alfy, a novel FYVE-domain-containing protein associated with protein granules and autophagic membranes." J Cell Sci 117(Pt 18): 4239-4251.

Smith, D. B. and K. S. Johnson (1988). "Single-step purification of polypeptides expressed in Escherichia coli as fusions with glutathione S-transferase." Gene 67(1): 31-40.

Smith, T. F., C. Gaitatzes, et al. (1999). "The WD repeat: a common architecture for diverse functions." Trends Biochem Sci 24(5): 181-185.

Sou, Y. S., S. Waguri, et al. (2008). "The Atg8 conjugation system is indispensable for proper development of autophagic isolation membranes in mice." Mol Biol Cell 19(11): 4762-4775.

Stenmark, H. (2009). "Rab GTPases as coordinators of vesicle traffic." Nat Rev Mol Cell Biol 10(8): 513-525.

Stromhaug, P. E., F. Reggiori, et al. (2004). "Atg21 is a phosphoinositide binding protein required for efficient lipidation and localization of Atg8 during uptake of aminopeptidase I by selective autophagy." Mol Biol Cell 15(8): 3553-3566.

Studier, F. W. (2005). "Protein production by auto-induction in high density shaking cultures." Protein Expr Purif 41(1): 207-234.

Studier, F. W. and B. A. Moffatt (1986). "Use of bacteriophage T7 RNA polymerase to direct selective high-level expression of cloned genes." J Mol Biol 189(1): 113-130.

Studier, F. W., A. H. Rosenberg, et al. (1990). "Use of T7 RNA polymerase to direct expression of cloned genes." Methods Enzymol 185: 60-89.

Sugawara, K., N. N. Suzuki, et al. (2005). "Structural basis for the specificity and catalysis of human Atg4B responsible for mammalian autophagy." J Biol Chem 280(48): 40058-40065.

Suzuki, K., T. Kirisako, et al. (2001). "The pre-autophagosomal structure organized by concerted functions of APG genes is essential for autophagosome formation." EMBO J 20(21): 5971-5981.

Suzuki, K., Y. Kubota, et al. (2007a). "Hierarchy of Atg proteins in pre-autophagosomal structure organization." Genes Cells 12(2): 209-218.

Suzuki, K. and Y. Ohsumi (2007b). "Molecular machinery of autophagosome formation in yeast, Saccharomyces cerevisiae." FEBS Lett 581(11): 2156-2161.

Suzuki, K. and Y. Ohsumi (2010). "Current knowledge of the pre-autophagosomal structure (PAS)." FEBS Lett 584(7): 1280-1286.

Suzuki, N. N., K. Yoshimoto, et al. (2005). "The crystal structure of plant ATG12 and its biological implication in autophagy." Autophagy 1(2): 119-126.

Taguchi-Atarashi, N., M. Hamasaki, et al. (2010). "Modulation of local PtdIns3P levels by the PI phosphatase MTMR3 regulates constitutive autophagy." Traffic 11(4): 468-478.

Takagi, T. and R. F. Doolittle (1974). "Amino acid sequence studies on factor XIII and the peptide released during its activation by thrombin." Biochemistry 13(4): 750-756.

Takeshige, K., M. Baba, et al. (1992). "Autophagy in yeast demonstrated with proteinasedeficient mutants and conditions for its induction." J Cell Biol 119(2): 301-311. 
Tanida, I., N. Mizushima, et al. (1999). "Apg7p/Cvt2p: A novel protein-activating enzyme essential for autophagy." Mol Biol Cell 10(5): 1367-1379.

Tanida, I., Y. S. Sou, et al. (2004). "HsAtg4B/HsApg4B/autophagin-1 cleaves the carboxyl termini of three human Atg8 homologues and delipidates microtubuleassociated protein light chain 3- and GABAA receptor-associated protein-phospholipid conjugates." J Biol Chem 279(35): 36268-36276.

Tanida, I., E. Tanida-Miyake, et al. (2002). "Human Apg3p/Aut1p homologue is an authentic E2 enzyme for multiple substrates, GATE-16, GABARAP, and MAP-LC3, and facilitates the conjugation of hApg12p to hApg5p." Journal of Biological Chemistry 277(16): 13739-13744.

Tanida, I., E. Tanida-Miyake, et al. (2001). "The human homolog of Saccharomyces cerevisiae Apg7p is a Protein-activating enzyme for multiple substrates including human Apg12p, GATE-16, GABARAP, and MAP-LC3." J Biol Chem 276(3): 17011706.

Thumm, M., R. Egner, et al. (1994). "Isolation of autophagocytosis mutants of Saccharomyces cerevisiae." FEBS Lett 349(2): 275-280.

Thumm, M. and D. J. Klionsky (2011). "New thoughts regarding Atg8 and ubiquitination." Autophagy 7(2): 125-126.

Towbin, H., T. Staehelin, et al. (1979). "Electrophoretic transfer of proteins from polyacrylamide gels to nitrocellulose sheets: procedure and some applications." Proc Natl Acad Sci U S A 76(9): 4350-4354.

Towbin, H., T. Staehelin, et al. (1989). "Immunoblotting in the clinical laboratory." J Clin Chem Clin Biochem 27(8): 495-501.

Travassos, L. H., L. A. Carneiro, et al. (2010). "Nod1 and Nod2 direct autophagy by recruiting ATG16L1 to the plasma membrane at the site of bacterial entry." Nat Immunol 11(1): 55-62.

Tresse, E., F. A. Salomons, et al. (2010). "VCP/p97 is essential for maturation of ubiquitin-containing autophagosomes and this function is impaired by mutations that cause IBMPFD." Autophagy 6(2): 217-227.

Tsukada, M. and Y. Ohsumi (1993). "Isolation and characterization of autophagydefective mutants of Saccharomyces cerevisiae." FEBS Lett 333(1-2): 169-174.

Tucker, K. A., F. Reggiori, et al. (2003). "Atg23 is essential for the cytoplasm to vacuole targeting pathway and efficient autophagy but not pexophagy." J Biol Chem 278(48): 48445-48452.

Vagin, A. and A. Teplyakov (1997). "Molecular replacement with MOLREP." Acta Crystallogr D Biol Crystallogr 66(Pt 1): 22-25.

van der Vaart, A., J. Griffith, et al. (2010). "Exit from the Golgi is required for the expansion of the autophagosomal phagophore in yeast Saccharomyces cerevisiae." Mol Biol Cell 21(13): 2270-2284.

Vergne, I., E. Roberts, et al. (2009). "Control of autophagy initiation by phosphoinositide 3-phosphatase Jumpy." EMBO J 28(15): 2244-2258.

Vicinanza, M., G. D'Angelo, et al. (2008). "Phosphoinositides as regulators of membrane trafficking in health and disease." Cell Mol Life Sci 65(18): 2833-2841. 
Wang, C. W., J. Kim, et al. (2001). "Apg2 is a novel protein required for the cytoplasm to vacuole targeting, autophagy, and pexophagy pathways." J Biol Chem 276(32): 3044230451 .

Wang, H., F. K. Bedford, et al. (1999). "GABA(A)-receptor-associated protein links GABA(A) receptors and the cytoskeleton." Nature 397(6714): 69-72.

Waring, M. J. (1965). "Complex formation between ethidium bromide and nucleic acids." J Mol Biol 13(1): 269-282.

Waugh, D. S. (2005). "Making the most of affinity tags." Trends Biotechnol 23(6): 316320.

Williams, A., S. Sarkar, et al. (2008). "Novel targets for Huntington's disease in an mTOR-independent autophagy pathway." Nat Chem Biol 4(5): 295-305.

$\mathrm{Wu}, \mathrm{M}$., T. Wang, et al. (2005). "Structural basis for recruitment of RILP by small GTPase Rab7." EMBO J 24(8): 1491-1501.

Wullschleger, S., R. Loewith, et al. (2006). "TOR signaling in growth and metabolism." Cell 124(3): 471-484.

Xia, H. G., L. Zhang, et al. (2010). "Control of basal autophagy by calpain1 mediated cleavage of ATG5." Autophagy 6(1): 61-66.

Xie, Z. and D. J. Klionsky (2007). "Autophagosome formation: core machinery and adaptations." Nat Cell Biol 9(10): 1102-1109.

Xie, Z., U. Nair, et al. (2008). "Atg8 controls phagophore expansion during autophagosome formation." Mol Biol Cell 19(8): 3290-3298.

Yang, Z. and D. J. Klionsky (2009). "An overview of the molecular mechanism of autophagy." Curr Top Microbiol Immunol 335: 1-32.

Yen, W. L. and D. J. Klionsky (2008). "How to live long and prosper: autophagy, mitochondria, and aging." Physiology (Bethesda) 23: 248-262.

Yen, W. L., J. E. Legakis, et al. (2007). "Atg27 is required for autophagy-dependent cycling of Atg9." Mol Biol Cell 18(2): 581-593.

Yen, W. L., T. Shintani, et al. (2010). "The conserved oligomeric Golgi complex is involved in double-membrane vesicle formation during autophagy." J Cell Biol 188(1): 101-114.

Yla-Anttila, P., H. Vihinen, et al. (2009). "3D tomography reveals connections between the phagophore and endoplasmic reticulum." Autophagy 5(8): 1180-1185.

Yorimitsu, T. and D. J. Klionsky (2005). "Atg11 links cargo to the vesicle-forming machinery in the cytoplasm to vacuole targeting pathway." Mol Biol Cell 16(4): 15931605.

Young, A. R., E. Y. Chan, et al. (2006). "Starvation and ULK1-dependent cycling of mammalian Atg9 between the TGN and endosomes." J Cell Sci 119(Pt 18): 38883900 .

Yousefi, S., R. Perozzo, et al. (2006). "Calpain-mediated cleavage of Atg5 switches autophagy to apoptosis." Nat Cell Biol 8(10): 1124-1132.

Yuan, W., P. E. Stromhaug, et al. (1999). "Glucose-induced autophagy of peroxisomes in Pichia pastoris requires a unique E1-like protein." Mol Biol Cell 10(5): 1353-1366. 
Yue, Z., S. Jin, et al. (2003). "Beclin 1, an autophagy gene essential for early embryonic development, is a haploinsufficient tumor suppressor." Proc Natl Acad Sci U S A 100(25): 15077-15082.

Zhang, L., J. Yu, et al. (2007). "Small molecule regulators of autophagy identified by an image-based high-throughput screen." Proc Natl Acad Sci U S A 104(48): 19023 19028.

Zheng, J. Y., T. Koda, et al. (1998). "A novel Rab GTPase, Rab33B, is ubiquitously expressed and localized to the medial Golgi cisternae." J Cell Sci 111 ( Pt 8): 10611069.

Zhou, F., Y. Yang, et al. (2011). "Bcl-2 and Bcl-xL play important roles in the crosstalk between autophagy and apoptosis." FEBS J 278(3): 403-413.

Zhu, G., P. Zhai, et al. (2004). "Structural basis of Rab5-Rabaptin5 interaction in endocytosis." Nat Struct Mol Biol 11(10): 975-983. 


\section{ACKNOWLEDGMENTS}

There are many people who have helped to make my $\mathrm{PhD}$ possible. I would firstly like to thank Dr. Karin Kühnel for giving me the exciting opportunity to be a member of her group. As a newcomer to the field, she deserves all the credit for training me in protein purification and allowing me to try my hand at crystallography with my own proteins. Furthermore, her door has always been open and her continuous enthusiasm and involvement in the project has been greatly appreciated.

I would also like to express my gratitude to Prof. Dr. Reinhard Jahn for giving me a place in his department where the equipment and resources have made this project possible. I would like to thank the people in the department for their support, especially those who have introduced me to specific techniques and equipment including Dr. Geert van den Bogaart for liposome preparation and flotation assays, Matias Hernandez for MALLS, and members of the Fasshauer group for ITC. I would also like to thank Michaela Hellwig, our technician and also the lab rotation students Sebastian Morales and Adi Bar Shalom. My fellow group member Ricarda Busse has been an appreciated discussion partner and played the special role of helping me to achieve my goal of becoming fluent in German during my time abroad.

I would like to thank Prof. Dr. Markus Wahl, Dr. Vlad Pena, and Prof. Dr. Reinhard Lührmann for enabling me to continue my interest in crystallization by providing me with access to their fantastic crystallization setup and facility. I cannot mention crystallography without also showing appreciation to Prof. George Sheldrick who was responsible for introducing me to x-ray crystallography. Additionally, this $\mathrm{PhD}$ would not have been possible without the generosity of Prof. Dr. Jäckle, who granted me bench space in his department for the first year of my $\mathrm{PhD}$.

I am also grateful for the time my thesis committee members, Prof. Dr. Michael Thumm and Prof. Dr. Markus Wahl, have spent with me over the years offering their expertise and practical suggestions. I would also like to thank Dr. Steffen Burkhardt, the coordinator for the IMPRS for Molecular Biology, for his constant support related to organizational issues. I am indebted to the IMPRS for their financial support of my project in the form of a $\mathrm{PhD}$ stipend throughout the length of my project. 
Lastly, I offer my heartfelt gratitude to my wonderful family for supporting my dream to go Europe and complete my $\mathrm{PhD}$ here in Göttingen, Germany. The opportunity was one that I could not pass up and they were behind me all the way, even while knowing what a sacrifice it would be. My heart was warmed with every card, letter, phone call, and care package and they made sure I knew that even though I was far away, I was constantly in their thoughts. I would also like to thank my sister for undertaking the brave task of transporting my cat across the ocean. For people who know me well, home for me is where the cat is. In addition to my family on the other side of the ocean, I am grateful to the McCallions, my American family here in Germany.

There are many more people who had an impact on my $\mathrm{PhD}$ time in Göttingen. They know who they are and how fortunate I feel for having them as a part of my life here. 


\section{Curriculum Vitae}

\section{Amanda M. Schalk, MSc}

Hospitalstr. 33c

37073 Goettingen, Germany

Tel +49(176)61161126

80 Henry St.

Belleville, MI 48111, USA

Tel +1(734)325-2394

\section{EDUCATION}

$\mathrm{PhD}$

Max Planck Institute for Biophysical Chemistry/Georg August

University Goettingen: Goettingen, Germany

Molecular Biology, expected May 2011

THESIS: Structural and functional characterization of the autophagy

proteins Atg5 and Atg16L1 and their interaction partners

MSc International Max Planck Research School/Georg August University

Goettingen: Goettingen, Germany

Molecular Biology, May 2008

THESIS: Characterization and structural determination of the mammalian Atg5-Atg16 and Atg5-FADD complexes

Final Grade $=1.83[$ German grading system $=$ A- $]$

BS

Eastern Michigan University: Ypsilanti, MI, USA

Professional Biochemistry; May 2006

THESIS: Synthesis of Pyrrolidines and Pyrrolizidines using the AZA-COPE

Rearrangement Mannich Cyclization Under Microwave Conditions

Departmental Honors in Chemistry

Final Grade = 3.8 Magna Cum Laude

High School Belleville High School: Belleville, MI, USA

May 2002

Graduated with Honors

Class Salutatorian $\left(2^{\text {nd }}\right.$ highest ranking in class of over 300$)$ 


\section{SCHOLARSHIPS AND AWARDS}

Belleville High School Salutatorian class of 2002 (over 300 students)

Full-ride Presidential Scholarship (Highest attainable scholarship at the university. Covered four years of full-time tuition, all fees, and room \& board). Eastern Michigan University 20022006

Valedictorian/Salutatorian Scholarship, Eastern Michigan University, 2002

Ronald Collins Endowed Scholarship for Undergraduate Research,

Eastern Michigan University, Fall 2005

Undergraduate Honors Fellowship, Eastern Michigan University,

3 semesters (Winter 2005-Winter 2006)

Honors Thesis Award, Eastern Michigan University, 2006

Dean's List of Eastern Michigan University College of Arts and Sciences (2002 - 2006)

International Max Planck Research School MSc Stipend, 2006-2007

Göttingen, Germany

International Max Planck Research School PhD Stipend, 2007-present

\section{ADVANCED AND SOFT SKILLS COURSES}

\section{BIOXHIT/BCO X-ray Crystallography}

Remote data collection at the ESRF and the CCP4 structure determination pipeline.

Lectures and tutorials given by Dr. Kevin Cowtan, Dr. Martyn Winn, Dr. Garib

Murshudov, Dr. Paul Emsley, Dr. Ian Berry, and Dr. Xavier Thibault: Oulu, Finnland, 21-

25 April 2008.

Crystal Structure Determination

Advanced x-ray crystallography lecture series with exercises given by Dr. George Sheldrick:

Göttingen,15 April - 7 July, 2009.

Isothermal Titration Calorimetry

Advanced method course using the Microcal ITC200 given by Prof. Dr. Kai Tittman at the

Göttingen Center for Molecular Biosciences: Göttingen, 23-24 March 2009.

Scientific Communication

Analyzed elements of effective presentations, posters, and scientific papers. Facilitated by

Dr. Heather Silyn-Roberts, author of "Writing for Science and Engineering: Papers,

Presentations and Reports": Göttingen, 2-4 December 2009.

Leadership \& Negotiations

Discussed effective leadership styles and negotation techniques. Facilitated by Elisabeth

Schick, author of "The I Factor, Success through Self-marketing”: Göttingen, 24 April 2009.

Professional Skills

Trained effects of voice, body language and negotiation techniques. Facilitated by Marion

Knaths, author of "Playing with power: How women can push through": Göttingen, 27

March 2009.

Powerspeak

Voice training course facilitated by Evelyn Gangl, a professional trainer for public speaking:

Göttingen, 22 June 2009. 


\section{POSTER PRESENTATIONS}

Characterization and Structural Determination of Atg5-FADD and Atg5-Atg16L1 Complexes (poster presentation), Gordon Research Conference "Autophagy in Stress, Development \& Disease”: 28-29 April 2010 in Barga (Lucca), Italy.

Structure Determination of the Mammalian Atg5-Atg16 and Atg16-Rab33B Complexes (poster presentation), $6^{\text {th }}$ Horizons in Molecular Biology International PhD Student Symposium: 10 September 2009 in Goettingen, Germany.

Application of a microwave assisted aza-Cope rearrangement-Mannich reaction to the synthesis of pyrrolizidine alkaloids (poster presentation), American Chemical Society spring national meeting: 27 March 2006 in Atlanta, Georgia.

Application of a microwave assisted aza-Cope rearrangement_Mannich reaction to the synthesis of pyrrolizidine alkaloids, Undergraduate Symposium at Eastern Michigan University: 31 March 2006 in Ypsilanti, Michigan.

Eastern Michigan University American Chemical Society Student Affiliate Activities at American

Chemical Society spring national meeting: 27 March 2006 in Atlanta, Georgia.

Use of a Microwave Oven to Shorten Reaction Times in the Synthesis of Five-Membered Rings, 2005

Undergraduate Symposium at Eastern Michigan University: 1 April 2005 in Ypsilanti, Michigan.

\section{ORAL PRESENTATIONS}

Atg5-Atg16 Phosphoinositide binding, 12 ${ }^{\text {th }}$ Molecular Biology PhD Seminar/Retreat: 10 April 2010 in Bad Arolsen, Germany.

Characterization and Structural Determination of Mammalian Atg5-Atg16 Complexes (oral presentation), 10 ${ }^{\text {th }}$ Molecular Biology PhD Block Seminar/Retreat: 28 March 2009 in Einbeck, Germany.

Characterization of the Mammalian Atg5-Atg16 and Atg5-FADD Complexes (oral presentation), $8^{\text {th }}$ Molecular Biology PhD Block Seminar/Retreat: 11 April 2008 in Teistungenburg, Germany.

\section{CO-CURRICULAR ACTIVITIES}

Co-organizer of the $6^{\text {th }}$ Horizons in Molecular Biology International PhD Student Symposium: 9-12 ${ }^{\text {th }}$ September 2009 in Göttingen, Germany.

Co-organizer of the $5^{\text {th }}$ Horizons in Molecular Biology International PhD Student Symposium: $10-13^{\text {th }}$ September 2008 in Göttingen, Germany.

President of Eastern Michigan University Chemistry Club: Fall 2005-Winter 2006

Treasurer of Eastern Michigan University Chemistry Club: Fall 2004-Winter 2005

Performed chemistry demonstrations at local middle and elementary schools as well as on Family

Day at Eastern Michigan University with Eastern Michigan University Chemistry Club.

Worked one-on-one with elderly with Alzheimer's and dementia at Huron Woods, an affiliate of St. Joseph Mercy Hospital in Ann Arbor, Michigan, USA.

Volunteered by serving food to the homeless at a soup kitchen in Ann Arbor, Michigan, USA 